\title{
MULTISPECTRAL SATELLITE IMAGE UNDERSTANDING
}

\section{DISSERTATION}

\author{
Presented in Partial Fulfillment of the Requirements for \\ the Degree Doctor of Philosophy in the \\ Graduate School of The Ohio State University \\ By
}

Cem Ünsalan, BSc. in EE, MSc. in EE $* * * * *$

The Ohio State University

2003

Dissertation Committee:

Approved by

Kim L. Boyer, PhD, Adviser

Stanley C. Ahalt, $\mathrm{PhD}$

Ashok Krishnamurthy, PhD

Adviser

Richard A. Miller, PhD

Adviser
Department of Electrical
Engineering


(c) Copyright by

Cem Ünsalan

2003 


\section{ABSTRACT}

A problem of major interest to regional planning organizations, disaster relief agencies, and the military is the identification and tracking of land development across large scale regions, and over time. We develop an autonomous image analysis system to understand land development, especially residential and urban building organizations from satellite images.

We introduce a set of measures based on straight lines to assess land development levels in high resolution satellite images. Urban areas exhibit a preponderance of straight line features. Rural areas produce line structures in more random spatial arrangements. We use this observation to perform an initial triage on the image to restrict the attention of subsequent, more computationally intensive analyses.

Vegetation indices have been used extensively to estimate the vegetation density from satellite and airborne images for many years. We use these as the multispectral information for classification and house and road extraction. We focus on the normalized difference vegetation index $(N D V I)$ and introduce a statistical framework to analyze and extend it. Using the established statistical framework, we introduce new a group of shadow-water indices.

We then extend our straight line based measures by developing a synergistic approach that combines structural and multispectral information. In particular, the structural features serve as cue regions for multispectral features. 
After the initial classification of regions, we introduce computationally more expensive but more precise graph theoretical measures over grayscale images to detect residential regions. The graphs are constructed using lines as vertices, while graph edges encode their spatial relationships. We introduce a set of measures based on various properties of the graph. These measures are monotonic with increasing structure (organization) in the image. We present a theoretical basis for the measures.

Having detected the residential regions, we introduce a novel system to detect houses and street networks in these. We extensively use the multispectral information and graph theory to extract houses and road networks.

We evaluated the performance of each step statistically and obtained very promising results. Especially, detection performances in house and street detection in residential regions is noteworthy. These results indicate the functionality of our satellite image understanding system. 
To my family. 


\title{
VITA
}

\author{
January 21,1973 .................... Born - Adana, Turkey \\ $1995 \ldots \ldots \ldots \ldots \ldots \ldots \ldots \ldots \ldots \ldots \ldots \ldots \ldots \ldots \ldots \ldots \ldots \ldots$.Sc. Electronics Engineering, \\ Hacettepe University \\ 1998 ................................. Electronics Engineering, \\ Boğaziçi University \\ 1999-present ........................ Graduate Research Associate, \\ SAMPL, The Ohio State University.
}

\section{PUBLICATIONS}

\section{Research Publications}

Cem Ünsalan and A. Erçil "Conversions between parametric and implicit forms". Computer Vision and Image Understanding, vol. 81, pp. 1-25, January 2001.

O. Kaynak, F. Kerestecioğlu, M. Ö. Efe and C. Cem Ünsalan (Eds.) "Proceedings of the Second International Conference on Recent Advances in Mechatronics (ICRAM'99)". Boğaziçi University Publications, No: 656, 1999.

Cem Ünsalan and A. Erçil "Automatic inspection of steel structures". O. Kaynak, S. Tosunoglu, M. Ang Jr. (Eds.), Recent Advances in Mechatronics, pp. 468-480, Springer-Verlag Ltd., Singapore, 1999

G. Downing, D. Chamberlain, A. Erçil and Cem Ünsalan "Robot vision beyond 2000". Proceedings of 29th International Symposium on Robotics, pp. 301-308, April 1998, Birmingham, UK

Cem Ünsalan and A. Erçil "A new robust and fast implicit polynomial fitting technique”. Proceedings of M2VIP'99, September 1999, Ankara, Turkey 
Cem Ünsalan and A. Erçil "Automated tolerance inspection by implicit polynomials". Proceedings of ICIAP'99, pp. 1218-1225, September 1999,Venice, Italy

Cem Ünsalan and A. Erçil "Conversion between parametric and implicit forms for computer graphics and vision". Proceedings of ISCIS'99, pp. 916-923, October 1999, Kusadasi, Turkey

Cem Ünsalan and A. Erçil "Shapes of features and a modified measure for linear discriminant analysis". Proceedings of ICPR2000, vol. 2, pp. 410-413, September 2000, Barcelona, Spain

Cem Ünsalan and K. L. Boyer "Classifying land development in high resolution satellite images using straight line statistics". Proceedings of ICPR2002, vol. 1, pp. 127-130, August 2002, Quebec City, Canada

Cem Ünsalan and K. L. Boyer "Linearized vegetation indices using a formal statistical framework". Proceedings of IGARRS03, (accepted for publication), Toulouse, France

\section{FIELDS OF STUDY}

Major Field: Electrical Engineering

Studies in:

Computer Vision Prof. Kim L. Boyer

Digital Signal Processing

Control Systems 


\section{TABLE OF CONTENTS}

Page

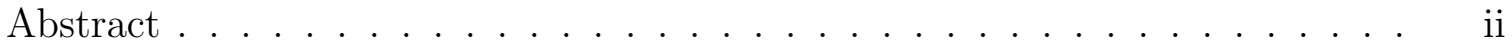

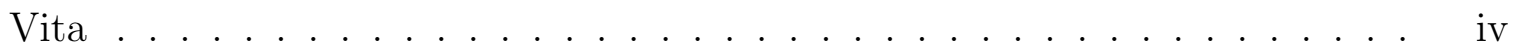

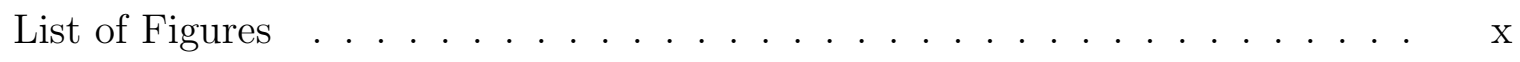

List of Tables . . . . . . . . . . . . . . . . xiv Chapters:

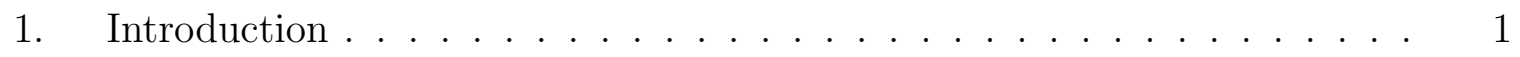

2. Land Use Classification: A Review . . . . . . . . . . . . . . 6

2.1 Remote Sensing Satellites and Airborne Sensors . . . . . . . . 7

2.1 .1 Landsat . . . . . . . . . . . . . . . . . . 8

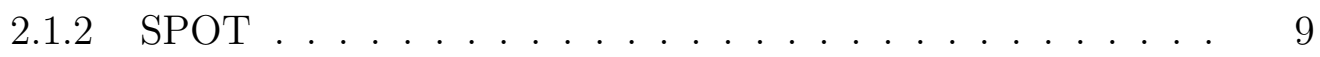

$2.1 .3 \quad$ IRS $\ldots \ldots \ldots \ldots \ldots \ldots \ldots$

2.1 .4 AVHRR . . . . . . . . . . . . . . . . . . . 11

2.1 .5 IKONOS . . . . . . . . . . . . . . . . . . . . . 12

2.1 .6 Airborne Image Sensors _ . . . . . . . . . . . . . . . . 12

2.1.7 Summary of the Sensors . . . . . . . . . . . . . . . . 13

2.2 Overview of Feature Extraction Methods . . . . . . . . . . . 16

2.3 Basic Feature Extraction Methods . . . . . . . . . . . . 17

2.3.1 Pixel Based Methods . . . . . . . . . . . . . . . . . . . 17

2.3.2 Texture Analysis Based Methods . . . . . . . . . . . . . . 19

2.4 Methods Using Contextual Information . . . . . . . . . . . . . . 21

2.4 .1 Spatial Coherence . . . . . . . . . . . . . . . . . . 21

2.4.2 Markov Random Fields . . . . . . . . . . . . . . . . 24 
2.4.3 Geographical Information Systems . . . . . . . . . . . 25

2.4.4 Expert Systems . . . . . . . . . . . . . . . . 26

2.5 Methods Summarizing Multidimensional Information . . . . . . . . 28

2.5.1 Data Dimensionality Reduction . . . . . . . . . . . . 28

2.5.2 Data and Decision Fusion . . . . . . . . . . . . . . 31

2.5.3 Summary of the Methods . . . . . . . . . . . . . . 32

2.6 Summary of the Chapter . . . . . . . . . . . . . . 34

3. Land Classification . . . . . . . . . . . . . . . 35

3.1 Line Support Regions (LSR) and Straight Line Extraction . . . . . 36

3.2 Statistical Feature Extraction . . . . . . . . . . . . . . 42

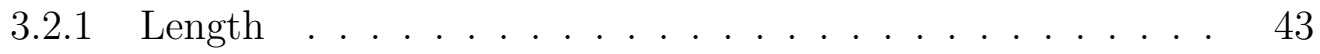

3.2 .2 Contrast........................... 44

3.3 Feature Based Grouping for Suburbia Detection . . . . . . . . . . . 46

3.4 Experimental Classification Results . . . . . . . . . . . . . 53

3.4.1 Dataset and Feature Space .............. 54

3.4 Two Class Results . . . . . . . . . . . . . . . 54

3.4.3 Suburban Area Detection Results . . . . . . . . . . . . . 56

3.5 Summary of the Overall Classification System . . . . . . . . . . . 58

3.6 Appendix: Additional Features . . . . . . . . . . . . . . 58

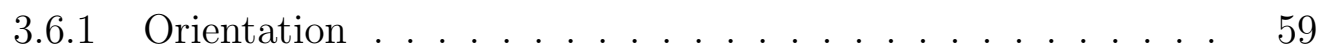

3.6.2 Line Spacing . . . . . . . . . . . . . . . . 60

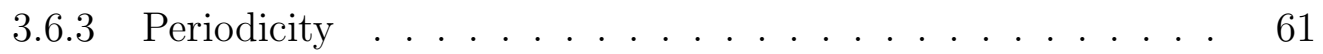

3.7 Appendix: More on Classification . . . . . . . . . . . . . 63

3.8 Summary of the Chapter . . . . . . . . . . . . . . 66

4. The Multispectral Information: Vegetation and Shadow-Water Indices . . 68

4.1 Background and Historical Development . . . . . . . . . . . . . . 70

4.2 Statistical Preliminaries . . . . . . . . . . . . . . . . 73

4.2.1 Principal Components Analysis (PCA) . . . . . . . . . 73

4.2.2 Parameter Estimation via Mode Matrix . . . . . . . . . . . 75

4.2.3 Entropy of a Distribution . . . . . . . . . . . . . . 75

4.3 Exploring the NDVI with a Statistical Framework . . . . . . . . 76

4.3.1 Estimating the PCA Parameters . . . . . . . . . . . 76

4.3.2 Statistical Construction of the NDVI . . . . . . . . . . . . 77

4.3.3 Saturation Problem of the NDVI . . . . . . . . . . . . 79

4.3.4 Experimental Results on the NDVI and $\theta$ Measure . . . . . 80

4.3.5 The Relationship Between the NDVI and RVI . . . . . . . . 87

4.4 New Vegetation Indices Based on the Statistical Framework . . . . 89 
4.4.1 A Vegetation Index Using Blue, Red, and Near - Infrared Bands . . . . . . . . . . . . . . . . . 9 90

4.4.2 A Vegetation Index Using Green, Red, and Near - Infrared Bands . . . . . . . . . . . . . . . . . . . 91

4.4.3 A Vegetation Index Using All Four Bands . . . . . . . . . . 92

4.5 Comparing the Vegetation Indices _. . . . . . . . . . . . . . 93

4.5.1 Comparison by the First Criterion (Visual Comparison and Dynamic Range) . . . . . . . . . . . . . . . . . . . 94

4.5.2 Comparison by the Second Criterion (Entropy on High Con-

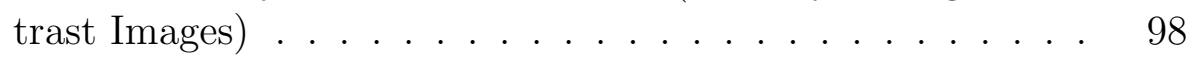

4.5.3 Comparison by the Third Criterion (Computational Cost) . 98

4.6 Shadow-Water Indices . . . . . . . . . . . . . . . . . . . . . . . . 99

4.7 Comparing the Shadow-Water Indices ． . . . . . . . . . . 101

4.7.1 Comparison by the First Criterion (Visual Comparison and Dynamic Range) . . . . . . . . . . . . . . . . . . . . 101

4.7.2 Comparison by the Second Criterion (Entropy on High Contrast Images $)$. . . . . . . . . . . . . . . . 106

4.8 Summary of the Chapter . . . . . . . . . . . . . . . 107

5. Land Use Classification via Multispectral Information . . . . . . . . . 108

5.1 Statistical Feature Extraction . . . . . . . . . . . . . . . . 109

$5.1 .1 \quad$ Structural Features . . . . . . . . . . . . . . . . . . . . 109

5.1 .2 Multispectral Features . . . . . . . . . . . . . . . . . 109

5.1 .3 Hybrid Features . . . . . . . . . . . . . . . . . . 110

5.2 Exploiting Spatial Coherence: Probabilistic Relaxation . . . . . . . 111

5.3 Experimental Classification Results . . . . . . . . . . . . . . 115

5.4 Appendix: Classification Performances . . . . . . . . . . . . . . 119

5.5 Summary of the Chapter . . . . . . . . . . . . . . . . . 120

6. Extracting Residential Regions by Graph Theoretical Measures . . . . . 124

6.1 Overview and Prior Work . . . . . . . . . . . . . . . 125

6.1 .1 Overview . . . . . . . . . . . . . . . . 125

6.1 .2 Prior Work . . . . . . . . . . . . . . . . 126

6.2 Graph Construction and Consensus Ordering . . . . . . . . . 127

6.3 Measures Based on Unweighted Graphs . . . . . . . . . . . . . 131

6.3 .1 Circuit Rank . . . . . . . . . . . . . . . . . . . . . 132

6.3 .2 The Degree Sequence . . . . . . . . . . . . . . . . . 132

6.4 Measures Based on Weighted Graphs . . . . . . . . . . . . . 136

6.4.1 Graph Partitioning by the Laplacian Cut . . . . . . . 138

6.4.2 Singular Values of the Adjacency Matrix . . . . . . . . . . . 141 
6.5 Fusing Measures . . . . . . . . . . . . . . . . . . . . . . 146

6.6 Experimental Results . . . . . . . . . . . . . . . . . . . . 147

6.6.1 Monotonicity: Comparison to Manual Evaluations . . . . . 147

6.6.2 Comparison with Sarkar and Boyer's Measures . . . . . . 150

6.6.3 Detecting Residential Regions . . . . . . . . . . . . . . 152

6.7 Summary of the Chapter . . . . . . . . . . . . . . . . . . . 161

7. House and Street Network Detection in Residential Regions . . . . . 162

7.1 Prior Work . . . . . . . . . . . . . . . . . . . 163

7.1 .1 Building Detection . . . . . . . . . . . . . . 163

7.1 .2 Road Detection . . . . . . . . . . . . . . . . 166

7.2 Using Multispectral Information . . . . . . . . . . . 168

7.2.1 A Derived Index to Detect Human Activity . . . . . . . . 168

7.2.2 Using a Shadow-Water Index to Eliminate Lakes . . . . . . 169

7.3 Segmenting the $\Omega$ Image . . . . . . . . . . . . . . . . . . . . 171

7.3.1 K-means Clustering with Spatial Coherence . . . . . . . . 172

7.3.2 Comparison with Other Methods . . . . . . . . . . . . . 173

7.3.3 Dependence on Initial Conditions . . . . . . . . . . . . . . 179

7.4 Shape Decomposition of the Foreground: The Binary Balloon Algo-

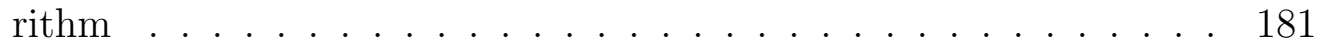

7.4.1 Constructing Initial Balloons . . . . . . . . . . . . . 182

7.4.2 Constructing the Binary Balloons . . . . . . . . . . . . . 184

7.4 .3 Combining Balloons via Voting . . . . . . . . . . . . . 187

7.4.4 Abstracting the Scene: Attributed Balloons . . . . . . . . 189

7.5 Street Network and House Detection . . . . . . . . . . . . . . 192

7.5.1 Eliminating Balloons Corresponding to Large Structures . . 192

7.5.2 Forming a Graph to Represent the Balloon Neighborhoods . 193

7.5 .3 The Detection Step . . . . . . . . . . . . . . . . . . . 195

7.5.4 Road Tracking by Prediction _. . . . . . . . . . . . 197

7.6 Results and Discussion . . . . . . . . . . . . . . . . . . . . 201

7.6.1 Pixel based Classification . . . . . . . . . . . . . . 201

7.6 .2 House Detection . . . . . . . . . . . . . . . . . . 201

7.6 .3 Street Network Detection . . . . . . . . . . . . . . . 202

7.6.4 Some Detection Examples . . . . . . . . . . . . . . 202

7.7 Summary of the Chapter . . . . . . . . . . . . . . . . . . . 209

8. Conclusions . . . . . . . . . . . . . . . . . . . . . . 210

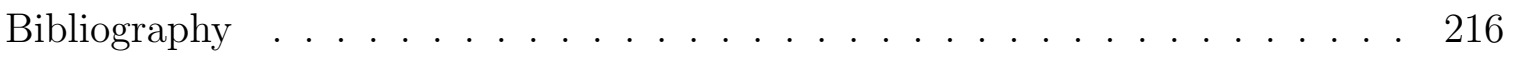




\section{LIST OF FIGURES}

Figure

Page

2.1 Sensor usage in three year time intervals from 1967 to 2002 . . . . . 15

2.2 Taxonomy of the feature extraction methods . . . . . . . . . . 16

2.3 Number of papers published in three year time intervals from 1967 to $2002 \ldots \ldots \ldots 33$

3.1 Impulse responses for the projection and edge detection filters . . . . 38

3.2 Sample subwindows and lines extracted from them . . . . . . . . . 41

3.3 Sample distribution in $\mu_{c}, E_{c}$ space . . . . . . . . . . . . . 47

3.4 The Colorado image and segments obtained from feature based grouping 49

3.5 The Sydney image and segments obtained from feature based grouping 50

3.6 The New Mexico image and segments obtained from feature based grouping . . . . . . . . . . . . . . . . . . . 51

3.7 The Ecuador image and segments obtained from feature based grouping 52

3.8 ROC curves for suburbia . . . . . . . . . . . . . . 57

3.9 An example of the projection of lines . . . . . . . . . . . 62

4.1 Scatter plot and eigenvectors for the NDVI . . . . . . . . . . . . 77

4.2 Rotation diagram for the PCA transformation . . . . . . . . 78 
4.3 The $N D V I$ and angle $\theta$ vs. LAI . . . . . . . . . . . . . . . . . 81

4.4 The Maryland image, $N D V I$ vs. $\theta$ representations . . . . . . . . . . . 82

4.5 The New Mexico image, $N D V I$ vs. $\theta$ representations . . . . . . . 83

4.6 The Minnesota image, $N D V I$ vs. $\theta$ representations . . . . . . . . 84

4.7 The Oregon image, $N D V I$ vs. $\theta$ representations . . . . . . . . . 85

4.8 Additional images for comparison based on entropy . . . . . . . . . . 86

4.9 Axis rotation by $\beta$ radians . . . . . . . . . . . . . . 88

4.10 Scatter plot and eigenvectors for the blue, red, and near-infrared bands 91

4.11 Scatter plot and eigenvectors for the green, red, and near-infrared bands 92

4.12 Comparison of vegetation indices on real images . . . . . . . . . 95

4.13 Comparison of histograms for the four vegetation indices to compare their dynamic ranges. ( solid $-\theta$, dot $-\theta_{2}$, dash and dot $-\theta_{3}$, dash $-\theta_{4}$ ) 96

4.14 Sample images to compare the four SWI . . . . . . . . . . . . . . 102

4.15 Color coded responses of the four SWI to the sample images . . . . . 103

4.16 Comparison of histograms for the four SWI to compare their dynamic range. ( solid $-\gamma_{1}$, dot $-\gamma_{2}$, dash and dot $-\gamma_{3}$, dash $\left.-\gamma_{4}\right) \ldots 105$

5.1 The Colorado Image, probabilistic relaxation iterations . . . . . . . . 114

5.2 The Rome Image, probabilistic relaxation results . . . . . . . . . . . 115

5.3 The Ecuador Image, probabilistic relaxation results . . . . . . . . . . 116

5.4 Improvements in classification for the Bayes, Parzen window, and NN classifiers. Dark bars correspond to urban performances, light bars correspond to average performances. . . . . . . . . . . . . 118

6.1 Sample test images arranged in increasing order by consensus ordering 129 
6.2 Lines obtained from the sample test images . . . . . . . . . . . . . . 130

6.3 Consensus ordering and the score distribution . . . . . . . . . . . 131

6.4 Sample degree sequence histograms and their best Poisson distribution fits. Filled circles - actual histograms, empty circles - Poisson distribution values. . . . . . . . . . . . . . . . 135

6.5 Normalized singular value distributions (solid curves) and corresponding approximations (dashed curves) for four sample images . . . . . . 145

6.6 Ordering of sample images based on all of our measures . . . . . . . . 149

6.7 Ordering of sample images based on three Sarkar Boyer measures . . 152

6.8 Histograms in 2D spaces, blue - rural region, black - residential region, red - urban region . . . . . . . . . . . . . . . . . 154

6.9 ROC curves for residential region detection . . . . . . . . . . . . 156

$7.1 \Omega$ example ....................... 170

7.2 Detecting water regions . . . . . . . . . . . . . . . 171

7.3 Test images and binary segments $C^{o}$ returned by Algorithm 2 - Part I 175

7.4 Test images and binary segments $C^{o}$ returned by Algorithm 2 - Part II 176

7.5 Comparison of segmentation algorithms . . . . . . . . . . . . 178

7.6 The Indiana image, segmentation results with different $t_{s}$ values . . 180

7.7 Extracting the binary balloon, an example . . . . . . . . . . . . 186

7.8 The South Dakota image, decomposition of its $C^{o} \ldots \ldots$. . . . . . 188

7.9 The South Dakota image, combination of balloons . . . . . . . . . . 189

7.10 Spine extraction example . . . . . . . . . . . . . . . . . . . 191 
7.11 The South Dakota image, street network extraction. Green sections are obtained by the graph theoretical method; red sections are obtained by road tracking . . . . . . . . . . . . . . . 200

7.12 The Indiana image, street network and house detection . . . . . . . 204

7.13 The Maryland image, street network and house detection . . . . . . 206

7.14 The South Dakota image, street network and house detection . . . . . 207

7.15 The South Dakota II image, street network and house detection . . . 208 


\section{LIST OF TABLES}

Table

Page

2.1 History of the Landsat family . . . . . . . . . . . . . . . 8

2.2 Landsat 7 spectral range . . . . . . . . . . . . . . . . . . 9

2.3 History of the SPOT family . . . . . . . . . . . . . 9

2.4 SPOT 5 spectral range . . . . . . . . . . . . . . 10

2.5 The history of the IRS family . . . . . . . . . . . . . . 10

2.6 IRS spectral range . . . . . . . . . . . . . . . . . . . . 11

2.7 History of the AVHRR family . . . . . . . . . . . . . . . . 11

2.8 History of the IKONOS family . . . . . . . . . . . . . 12

2.9 IKONOS spectral range . . . . . . . . . . . . . . . . 12

2.10 Airborne sensors . . . . . . . . . . . . . . . . . . . 13

2.11 Daedalus spectral range . . . . . . . . . . . . . . 13

2.12 Summary of the sensor properties . . . . . . . . . . . . . 14

2.13 Pixel based classification . . . . . . . . . . . . . . . . 19

2.14 Texture analysis based classification . . . . . . . . . . . . . . 21

2.15 Spatial information based classification . . . . . . . . . . 23 
2.16 MRF based classification . . . . . . . . . . . . . 25

2.17 GIS classifier improvements . . . . . . . . . . . . . 26

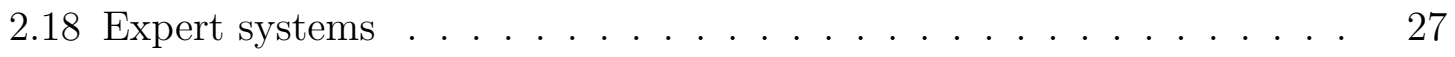

2.19 Optimal band selection . . . . . . . . . . . . . . . . . . . 29

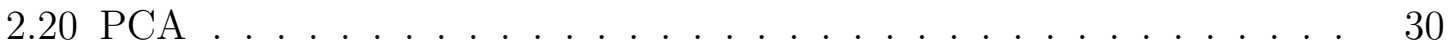

2.21 Spectral unmixing . . . . . . . . . . . . . . . . . . . 30

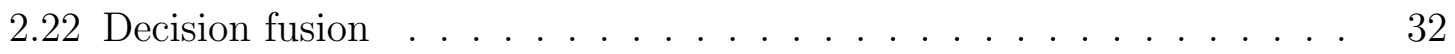

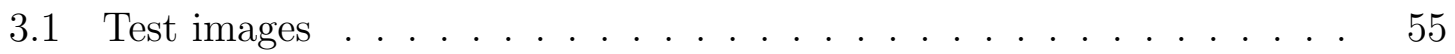

3.2 Summary of the system parameters . . . . . . . . . . . . 58

3.3 Performances for best feature spaces for the Bayes classifier . . . . . 63

3.4 Performances for best feature spaces for the Parzen window classifier 64

3.5 Performances for best feature spaces for the NN classifier . . . . . . 64

3.6 The Bayes classification results in percentages for all 1D and 2D feature

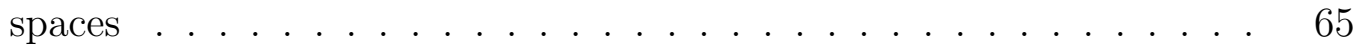

3.7 The Parzen window classification results in percentages for all 1D and 2D feature spaces . . . . . . . . . . . . . . . 65

3.8 The NN classification results in percentages for all $1 \mathrm{D}$ and $2 \mathrm{D}$ feature

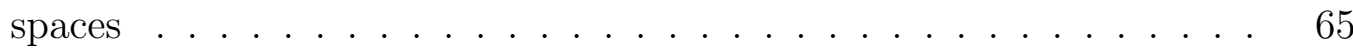

3.9 Comparison of performances for the three classifiers $\ldots \ldots \ldots 6$

4.1 Comparison of the entropies for the six images for the NDVI and $\theta \quad . \quad 87$

4.2 Comparison of the four vegetation indices on the Maryland and Oregon images . . . . . . . . . . . . . . . . . . 97

4.3 Comparison of the entropies on six images for the four vegetation indices 98 
4.4 Comparison of the four SWI on the New Mexico, and Florida images 104

4.5 Comparison of the entropies on three images for the four SWI . . . . 106

5.1 Test images . . . . . . . . . . . . . . . . . . . . . 121

5.2 Comparison of detection performances . . . . . . . . . . . . . . 122

5.3 Comparison of detection performances . . . . . . . . . . . . . . . . . 122

5.4 Comparison of performances for the Bayes classifier . . . . . . . . . . 122

5.5 Comparison of performances for the Parzen window classifier . . . . . 122

5.6 Comparison of performances for the NN classifier . . . . . . . . 123

6.1 Geographical locations and types of the sample test images . . . . . . 128

6.2 False alarms and deviations for measures . . . . . . . . . . . . . 148

6.3 False alarms and deviations for Sarkar Boyer measures . . . . . . . . 151

6.4 Area under ROC curves . . . . . . . . . . . . . . . . 157

6.5 Three class classification results on $\mu_{c}, E_{c}$ space . . . . . . . . . 158

6.6 Three class classification results on $E_{c}, m_{d s}$ space . . . . . . . . . 158

6.7 Three class classification results on $\mu_{c}, m_{d s}$ space $\ldots \ldots$. . . . . . . 159

6.8 Three class classification results in $\mu_{c}, m_{F}$ space . . . . . . . . . . 159

$6.9 \Delta$ classification on $\left(\mu_{c}, m_{d s}\right)$ - classification on $\left(\mu_{c}, E_{c}\right) \ldots 160$

7.1 Building detection review, in Mayer's format . . . . . . . . . . . . . 164

7.2 Building detection review, performances . . . . . . . . . 165

7.3 Road detection review . . . . . . . . . . . . . 166 
7.4 Road detection review, performances . . . . . . . . . . . . 167

7.5 Comparison of performances . . . . . . . . . . . . . . . . . . 179 


\section{List of Algorithms}

$1 \quad$ K-means clustering . . . . . . . . . . . . . . . . . 172

2 K-means clustering with spatial coherence . . . . . . . . . . . . 174

3 Algorithm to construct initial balloons . . . . . . . . . . . . . . 183

4 The binary balloon algorithm . . . . . . . . . . . . . 185

5 Dijkstra's shortest path algorithm . . . . . . . . . . . . 195

6 Street network and house detection algorithm . . . . . . . . . . . 198 


\section{CHAPTER 1}

\section{Introduction}

Cities are evolving and districts are changing their characteristics faster than ever before. Although the evolution is slow in the central parts of most cities, it is typically fairly fast in outlying regions. Yesterday's forested or rural regions around the city become tomorrow's residential regions (These observations are especially valid in North America). These changes cause many problems for policy makers and government agencies.

They affect the public and private utility networks. Maps become less reliable around these regions. As a result, emergency plans based on these maps become unreliable. They affect the public and private utility networks and maps become less reliable.

Konecny and Schiewe [1] summarize some facts regarding manual map generation. According to their analysis, $33.5 \%$ of the world was mapped at $1: 25000$ scale (around one meter per pixel resolution) as of 1993. This resolution is vital for mapping most mature cities (such as European cities) because their buildings and road networks are in close proximity. For this scale, the annual manual map generation rate is around 2.8\% Similarly, the annual manual map updating rate is around $4.9 \%$. Konecny and Schiewe summarize the urgency to automate map generation: 
" On average, maps of 1:25000-scale are 20 years out of date and 1:50000-scale sheets may be 40 (or more) years old."

Considering that a house can be built less than a year (and destroyed in minutes), and larger buildings can be built within two to three years, the inadequacy of current map updating rates is evident.

Besides affecting utility networks and maps, these changes also affect tax assessment information and demographics. The update rate of census information (10 years in the United States, for example) is insufficient to adequately track these changes.

While the advent of commercially available, high-resolution satellite imagery addresses the data collection issue, the rate at which these sensors provide data currently far exceeds the rate at which those data can be analyzed. To assist experts, planners, policy makers, and civil defense organizations, we are developing automated techniques.

Because the volume of data - and the size of individual images - is so great, we first focus on a "triage" in which urban and non-urban classes are first identified with (relatively) minimal processing. Therefore, we introduce a set of measures based on straight lines to assess land development levels in high resolution (one meter) satellite images. Urban areas exhibit a preponderance of straight line features, generally appearing in fairly simple, quasiperiodic organizations. Wilderness and rural areas produce line structures in more random spatial arrangements. We use this observation to perform an initial triage on the image to restrict the attention of subsequent, more computationally intensive analyses. Statistical measures based on straight lines guide the analysis. We base these measures on orientation, length, contrast, periodicity and location. 
We trained and tested parametric and non-parametric classifiers using this feature set. These tests were for a two-class problem (urban vs. rural). However, because our ultimate goal is to extract residential regions, we extended these ideas to address the detection of suburban regions. To do so, some use of spatial coherence is required; suburban regions are especially difficult to detect. Therefore, we introduce a decision system to perform suburban region classification via an overlapping voting method for consensus discovery.

We use vegetation indices as the multispectral information for classification and house and street network extraction. Vegetation indices have been used extensively to estimate the vegetation density from satellite and airborne images for many years. We first focus on one of the most popular vegetation indices, the normalized difference vegetation index $(N D V I)$ and introduce a statistical framework to analyze it. We propose a solution to the saturation problem of this index based on our statistical framework. As the vegetation density increases, the corresponding $N D V I$ values start to saturate and cannot represent highly vegetated regions reliably. By testing our method on real images, we show that we can obtain a linearized (and more reliable) measure than the NDVI.

We investigate the relationship of this index with the ratio vegetation index $(R V I)$, another popular vegetation index. As we know, the NDVI uses only red and nearinfrared bands. Using the established statistical framework, we introduce new vegetation indices using blue and green bands in addition to the red and the near-infrared. We compare these indices with the measure obtained from the $N D V I$ on real images and comment on the results. Finally, we introduce a group of shadow-water indices to measure the shadow and water density in multispectral satellite and airborne images. 
As we extend the vegetation indices, we used them to improve the two class classification (urban vs. rural). Therefore, we developed a synergistic approach that combines structural and multispectral information. In particular, the structural features serve as cue regions for multispectral features.

After the initial urban vs. rural classification of regions, we introduce computationally more expensive but more precise graph theoretical measures over grayscale images to detect residential regions. The graphs are constructed using lines (obtained from line support regions) as vertices, while graph edges encode their spatial relationships. We then introduce a set of measures based on various properties of the graph. These measures are monotonic with increasing structure (organization) in the image. Thus, increased cultural activity and land development are indicated by increases in these measures - without explicit extraction of street networks, buildings, residences etc. These latter, time consuming (and still only partially automated) tasks can be restricted only to "promising" image regions, according to our measures.

We present a theoretical basis for the measures followed by extensive experimental results in which the measures are first compared to manual evaluations of land development. We then present and test a method to focus on, and (pre)extract, suburban-style residential areas. These are of particular importance in many applications, and particularly difficult to extract.

Having detected the residential regions, we introduce a novel system to detect houses and street networks in these. Our system comprises four main parts. First, we introduce measures on multispectral images to detect regions of possible human activity. On these measures, we introduce a variation of the k-means clustering algorithm to extract possible houses and street networks by combining both spatial 
and spectral features. This combination of information improves the final clustering results. From clustering, we obtain a binary image containing possible street network fragments and houses. We then decompose this binary image using a balloon algorithm based on binary mathematical morphology.

Having obtained the decomposition, we represent them in a graph theoretical framework. Balloons serve as vertices and their neighborhood information is encoded by weighted edges in the graph. The street network is extracted from the graph by using the shortest paths algorithm. The remaining vertices (balloons) are assigned as possible houses in the region.

We evaluate the performance of each step statistically. For the initial urban vs. rural discrimination, we obtained a classification performance around $88 \%$ over 6000 test samples. For the vegetation indices, we experimentally showed that our proposed extension to the NDVI performs better than the NDVI. Besides, the newly introduced shadow-water indices proved to be useful in water and shadow detection. For the graph theoretical measures, we obtained a highly satisfactory monotonicity measures. With them, we also obtained classification performances around $89 \%$ to detect residential regions. Finally, we have a 93\% detection performance over 6803 houses; and a $95 \%$ detection performance for the $220 \mathrm{~km}$ road networks. These results indicate the functionality of our satellite image understanding system. 


\section{CHAPTER 2}

\section{Land Use Classification: A Review}

Land use classification covers a wide range of applications from general land cover determination to specific crop type detection. Many approaches have been developed to infer land usage from satellite and aerial images. In this chapter, we investigate trends in land use classification by reviewing the literature. We did not attempt to cover the whole of the literature; however, we tried to explore a significant and influential portion of it. Specifically, we focused on feature extraction methods using passive sensors and excluded work on classifiers, neural networks, and fuzzy logic. To identify trends, we grouped papers based on their major contribution. We summarize key papers in each group and tabulate each study by the type of image used, geographical location considered, and the average performance obtained. To clarify image types used in the literature, we include a section summarizing remote sensing satellites and airborne equipment.

There have been many methods proposed to solve these problems in terms of land use classification. We consider the seminal work of Fu et al. [2] to be the beginning of automated land use classification. To investigate the trends in solving this problem, we reviewed over 90 influential papers published in refereed journals. We grouped these papers according to their major contribution. One paper could belong to many 
groups, but we chose to assign each paper to only one "best" group. To limit the scope of the study, we focused on feature extraction methods based on passive sensors. We also excluded work using specifically designed classifiers, neural networks or fuzzy logic, although they provide another perspective.

Unfortunately, the results given in most of the papers we reviewed are based on just one (or a very few) images. It is rarely the case that one finds two papers using the same image(s) to evaluate their methods. For this reason, we were unable to compare the performances of these methods directly. Nevertheless, we report their performances to give the reader some idea of their relative performance. In reporting performance, we used the average classification rate as a benchmark. If the method was applied to several images, we report the mean of the average classification rate for each image.

In these studies, researchers used various satellite or airborne passive sensors. Each sensor has strengths and weaknesses depending on the spectrum it sweeps, the resolution, and the revisit interval. We begin with a review of these satellite and airborne sensors.

\subsection{Remote Sensing Satellites and Airborne Sensors}

There are various remote sensing satellites and airborne systems. In almost all cases, the sensors on these satellites and airborne systems are called by the same name as the satellite or the airborne program. We follow the same convention.

The remote sensing satellites (sensors) we consider are Landsat, SPOT, IRS, AVHRR, and IKONOS; the aerial sensors (programs) we consider are Daedalus, AVIRIS, HYDICE, and DAIS 7915. Wherever possible, we give a brief historical 
development of the sensor family with the operation dates, resolution, and revisit interval. We also give the spectral properties of the latest sensor for each family. We tabulate this information as a sensor selection guide. At the last section we summarize and compare the properties of these sensors and their usage through time to give a brief information to the potential user.

\subsubsection{Landsat}

One of the best known families of remote sensing satellites is Landsat. This is a US based sensor family that has evolved over time. The first satellite in this family, launched in 1972, was Landsat 1. It had two sensors, the Return Beam Vidicom (RBV) and the Multi Spectral Scanner (MSS). RBV was a television camera, since replaced by the Thematic Mapper (TM) in Landsats 4 and 5. In the last two satellites, there are panchromatic (pan), Enhanced Thematic Mapper (ETM) and Enhanced Thematic Mapper Plus (ETM+) sensors (Table 2.1). This family remains active; its average resolution is around 15 meters with a 16 day revisit interval. Table 2.2 summarizes the spectral properties of the latest family member, Landsat 7 .

\begin{tabular}{|l|r|r|l|c|}
\hline Satellite & Launch date & End of service & Resolution $(m)$ & Revisit (days) \\
\hline Landsat 1 & $7 / 23 / 1972$ & $1 / 6 / 1978$ & RBV 80; MSS 80 & 18 \\
\hline Landsat 2 & $1 / 22 / 1975$ & $2 / 25 / 1982$ & RBV 80; MSS 80 & 18 \\
\hline Landsat 3 & $3 / 5 / 1978$ & $3 / 31 / 1983$ & RBV 30; MSS 80 & 18 \\
\hline Landsat 4 & $7 / 16 / 1982$ & & TM 30; MSS 80 & 16 \\
\hline Landsat 5 & $3 / 1 / 1984$ & & TM 30; MSS 80 & 16 \\
\hline Landsat 6 & $10 / 5 / 1993$ & $10 / 5 / 1993$ & Pan 15; ETM 30 & 16 \\
\hline Landsat 7 & $4 / 15 / 1999$ & & Pan 15; ETM+ 30 & 16 \\
\hline
\end{tabular}

Table 2.1: History of the Landsat family 


\begin{tabular}{|c|c|c|}
\hline Band & Spectral range $(\mu \mathrm{m})$ & Resolution $(\mathrm{m})$ \\
\hline 1 & 0.450 to 0.515 & 30 \\
\hline 2 & 0.525 to 0.605 & 30 \\
\hline 3 & 0.630 to 0.690 & 30 \\
\hline 4 & 0.750 to 0.900 & 30 \\
\hline 5 & 1.550 to 1.750 & 30 \\
\hline 6 & 10.400 to 12.500 & 60 \\
\hline 7 & 2.090 to 2.350 & 30 \\
\hline Pan & 0.520 to 0.900 & 15 \\
\hline
\end{tabular}

Table 2.2: Landsat 7 spectral range

\subsubsection{SPOT}

SPOT is a French-Belgian-Swedish joint remote sensing satellite family. SPOT 1, launched in 1986, offered 10 meter panchromatic (pan) and 26 meter multispectral (ms) images with a 26 day revisit interval. The newest member of the family has the same revisit interval with 2.5 or 5 meter panchromatic and 10 meter multispectral image resolution (Table 2.3). See Table 2.4 for the spectral properties of SPOT 5, the latest SPOT in the sky.

\begin{tabular}{|l|r|r|c|}
\hline Satellite & Launch date & Resolution $(m)$ & Revisit (days) \\
\hline SPOT 1 & $2 / 22 / 1986$ & Pan 10; MS 20 & 26 \\
\hline SPOT 2 & $1 / 22 / 1990$ & Pan 10; MS 20 & 26 \\
\hline SPOT 3 & $9 / 26 / 1993$ & Pan 10; MS 20 & 26 \\
\hline SPOT 4 & $3 / 4 / 1998$ & Pan 10; MS 20 & 26 \\
\hline SPOT 5 & $5 / 4 / 2002$ & Pan 2.5 or 5; MS 10 & 26 \\
\hline
\end{tabular}

Table 2.3: History of the SPOT family 


\begin{tabular}{|c|c|c|}
\hline Band & Spectral range $(\mu \mathrm{m})$ & Resolution $(\mathrm{m})$ \\
\hline 1 & 0.50 to 0.59 & 10 \\
\hline 2 & 0.61 to 0.68 & 10 \\
\hline 3 & 0.78 to 0.89 & 10 \\
\hline 4 & 1.58 to 1.75 & 20 \\
\hline Pan & 0.48 to 0.71 & 2.5 or 5 \\
\hline
\end{tabular}

Table 2.4: SPOT 5 spectral range

\subsubsection{IRS}

IRS is the Indian remote sensing satellite family, first launched in 1988 with 72.5 meter resolution. Although other Indian remote sensing satellites were launched prior to this, this is first Indian family to see extensive use. The latest family member, IRS $2 \mathrm{~A}$, has 5 to 10 meter panchromatic and 23.5 to 70 meter multispectral image resolution (Table 2.5). The spectral properties of the latest family member appear in Table 2.6.

\begin{tabular}{|l|r|r|l|c|}
\hline Satellite & Launch date & End of service & Resolution $(m)$ & Revisit (days) \\
\hline IRS 1A & $3 / 17 / 1988$ & 1992 & 72.5 & 22 \\
\hline IRS 1B & $8 / 29 / 1991$ & 1999 & 72.5 & 22 \\
\hline IRS 1C & $12 / 28 / 1995$ & 1997 & Pan 5.8; MS 23.5 & 24 \\
\hline IRS 1D & $9 / 29 / 1997$ & 1997 & Pan 5.8; MS 23.5 & 24 \\
\hline IRS 2A & 2000 & & Pan 5-10; MS 23.5-70.5 & 24 \\
\hline IRS P2 & $10 / 15 / 1994$ & 1997 & Pan 5.8; MS 36.25 & 22 \\
\hline IRS P3 & $3 / 21 / 1996$ & 1997 & Pan 189; MS 523 & 5 \\
\hline
\end{tabular}

Table 2.5: The history of the IRS family 


\begin{tabular}{|c|c|c|}
\hline Band & Spectral range $(\mu \mathrm{m})$ & Resolution $(\mathrm{m})$ \\
\hline 1 & 0.50 to 0.59 & 23.5 \\
\hline 2 & 0.62 to 0.68 & 23.5 \\
\hline 3 & 0.77 to 0.86 & 23.5 \\
\hline 4 & 1.55 to 1.70 & 70.5 \\
\hline Pan & 0.50 to 0.75 & 5.8 \\
\hline
\end{tabular}

Table 2.6: IRS spectral range

\subsubsection{AVHRR}

Another US based sensor is the Advanced Very High Resolution Radiometer (AVHRR). This sensor family differs from previous sensors in both resolution and intended application. It has a fairly low resolution (around $1.1 \mathrm{~km}$ ) and is basically used for vegetation and forestry studies (Table 2.7). The latest AVHRR sensor, NOAA-16, has 6 bands covering the visible, near-infrared, and thermal infrared.

\begin{tabular}{|l|r|r|c|}
\hline Satellite & Launch date & End of service & Resolution $(\mathrm{km})$ \\
\hline NOAA-6 & $6 / 79$ & $11 / 86$ & 1.1 \\
\hline NOAA-7 & $8 / 81$ & $6 / 86$ & 1.1 \\
\hline NOAA-8 & $5 / 83$ & $10 / 85$ & 1.1 \\
\hline NOAA-9 & $2 / 85$ & $11 / 88$ & 1.1 \\
\hline NOAA-10 & $11 / 86$ & $9 / 91$ & 1.1 \\
\hline NOAA-11 & $11 / 88$ & $9 / 94$ & 1.1 \\
\hline NOAA-12 & $5 / 91$ & $12 / 94$ & 1.1 \\
\hline NOAA-14 & $12 / 94$ & & 1.1 \\
\hline NOAA-15 & $5 / 98$ & & 1.1 \\
\hline NOAA-16 & $9 / 00$ & & 1.1 \\
\hline
\end{tabular}

Table 2.7: History of the AVHRR family 


\subsubsection{IKONOS}

IKONOS is US based commercial remote sensing satellite. It has the highest image resolution publicly available, with one meter panchromatic and four meter multispectral images (Table 2.8). See Table 2.9 for the spectral properties of IKONOS 2.

\begin{tabular}{|l|r|c|l|c|}
\hline Satellite & Launch date & End of service & Resolution $(m)$ & Revisit (days) \\
\hline IKONOS 1 & $4 / 27 / 1999$ & $4 / 27 / 1999$ & Pan 1; MS 4 & 3 \\
\hline IKONOS 2 & $9 / 24 / 1999$ & & Pan 1; MS 4 & 3 \\
\hline
\end{tabular}

Table 2.8: History of the IKONOS family

\begin{tabular}{|c|c|c|}
\hline Band & Spectral range $(\mu m)$ & Resolution $(\mathrm{m})$ \\
\hline 1 & 0.45 to 0.52 & 4 \\
\hline 2 & 0.51 to 0.60 & 4 \\
\hline 3 & 0.63 to 0.70 & 4 \\
\hline 4 & 0.76 to 0.85 & 4 \\
\hline Pan & 0.45 to 0.90 & 1 \\
\hline
\end{tabular}

Table 2.9: IKONOS spectral range

\subsubsection{Airborne Image Sensors}

There are various airborne sensors available to supplement satellites for land classification. These sensors have resolutions comparable to satellite based sensors. Their superiority is the range of the spectrum they sweep and the number of spectral bands. 
However, they are limited by range and time. We summarize these sensors in Table 2.10 and give the spectral properties of the Daedalus sensor in Table 2.11.

\begin{tabular}{|l|c|c|r|}
\hline Program & Resolution $(m)$ & Spectral range $(\mu m)$ & Total \# of bands \\
\hline Daedalus & 25 & 0.42 to 14.00 & 12 \\
\hline DAIS 7915 & 3 to 20 & 0.40 to 12.60 & 79 \\
\hline HYDICE & 1 to 4 & 0.40 to 2.45 & 210 \\
\hline AVIRIS & 17 & 0.40 to 2.45 & 224 \\
\hline
\end{tabular}

Table 2.10: Airborne sensors

\begin{tabular}{|c|c|c|}
\hline Band & Spectral range $(\mu \mathrm{m})$ & Resolution $(m)$ \\
\hline 1 & 0.42 to 0.45 & 25 \\
\hline 2 & 0.45 to 0.52 & 25 \\
\hline 3 & 0.52 to 0.60 & 25 \\
\hline 4 & 0.60 to 0.62 & 25 \\
\hline 5 & 0.63 to 0.69 & 25 \\
\hline 6 & 0.69 to 0.75 & 25 \\
\hline 7 & 0.76 to 0.90 & 25 \\
\hline 8 & 0.91 to 1.05 & 25 \\
\hline 9 & 1.55 to 1.75 & 25 \\
\hline 10 & 2.08 to 2.35 & 25 \\
\hline 11 (High gain) & 8.50 to 14.00 & 25 \\
\hline 12 (Low gain) & 8.50 to 14.00 & 25 \\
\hline
\end{tabular}

Table 2.11: Daedalus spectral range

\subsubsection{Summary of the Sensors}

Although sensor information is probably known to most readers, we include them here for two main reasons. First, to give a review of the sensor technology. Second, most feature extraction methods directly depend on the resolution and spectrum the 
sensor offers. Therefore, an improvement in the sensor may lead to new methods in the land use classification research. Let's first summarize (Table 2.12) and compare the the latest family members of these sensors.

\begin{tabular}{|l|c|c|c|}
\hline Sensor & Resolution $(m)$ & Spectral range $(\mu m)$ & Revisit interval (days) \\
\hline Landsat & 15 & 0.45 to 2.35 & 16 \\
\hline SPOT & 2.5 & 0.50 to 1.75 & 5 \\
\hline IRS & 5 & 0.50 to 1.70 & 3 \\
\hline IKONOS & 1 & 0.45 to 0.85 & N $/ \mathrm{A}$ \\
\hline Airborne & 1 to 25 & 0.42 to 14.00 & \\
\hline
\end{tabular}

Table 2.12: Summary of the sensor properties

This table suggests the following conclusions. IKONOS and airborne sensors have the highest resolution. SPOT has the next highest resolution, followed by IRS and Landsat. If the resolution is the most important parameter in system design, IKONOS seems to be the best choice available.

However, these sensors also differ in spectral range. Airborne sensors in general has the widest spectral range available. Landsat, SPOT, IRS, and IKONOS follow them. Although airborne sensors offer superior spectral range, they suffer from range and time (as we mentioned previously). Therefore, Landsat is the optimal choice if the spectral and operational ranges are considered. However, if a higher spectral range is required, airborne sensors can be used for small geographic locations.

IKONOS is also superior in terms of revisit interval, followed by SPOT, IRS, and Landsat. For applications requiring frequent updates, IKONOS offers the best solution; Landsat the worst. Although IKONOS has a narrower spectral range than 
Landsat, its resolution and revisit interval makes this sensor attractive for most applications.

So, the potential user has a wide range of choices on the resolution, spectral range, and revisit interval he desires. Next, we group and count the papers published by the sensors they used in Fig. 2.1 in three year time intervals, starting from 1967 to 2002. This records the popularity of these sensors over time.

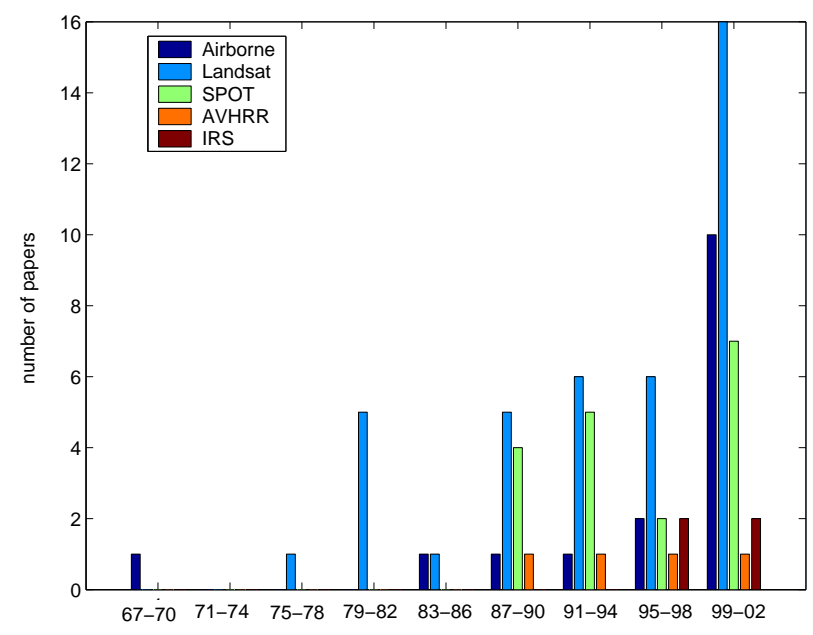

Figure 2.1: Sensor usage in three year time intervals from 1967 to 2002

This figure indicates the popularity of the Landsat family through time and there are many reasons for this popularity. One main reason is the high spectral range and moderate resolution Landsat offers. The other reason may be the availability of its images. Besides Landsat, airborne sensors and SPOT have also been used extensively. Since IKONOS has been launched recently, it is not shown here. However, based on the above comparison it offers great promise for future land use classification studies. 
The final, somewhat obvious, observation we can make is that overall use and study of remote sensing platforms shows near-exponential growth.

\subsection{Overview of Feature Extraction Methods}

Before explaining each feature extraction method in depth, we summarize them in a tree as in Fig. 2.2. Here, we group feature extraction methods into three main categories: basic methods, methods using contextual information, and methods summarizing the multidimensional information. In this table, we provide the time interval over which each method has been used and the sensors their results are reported on.

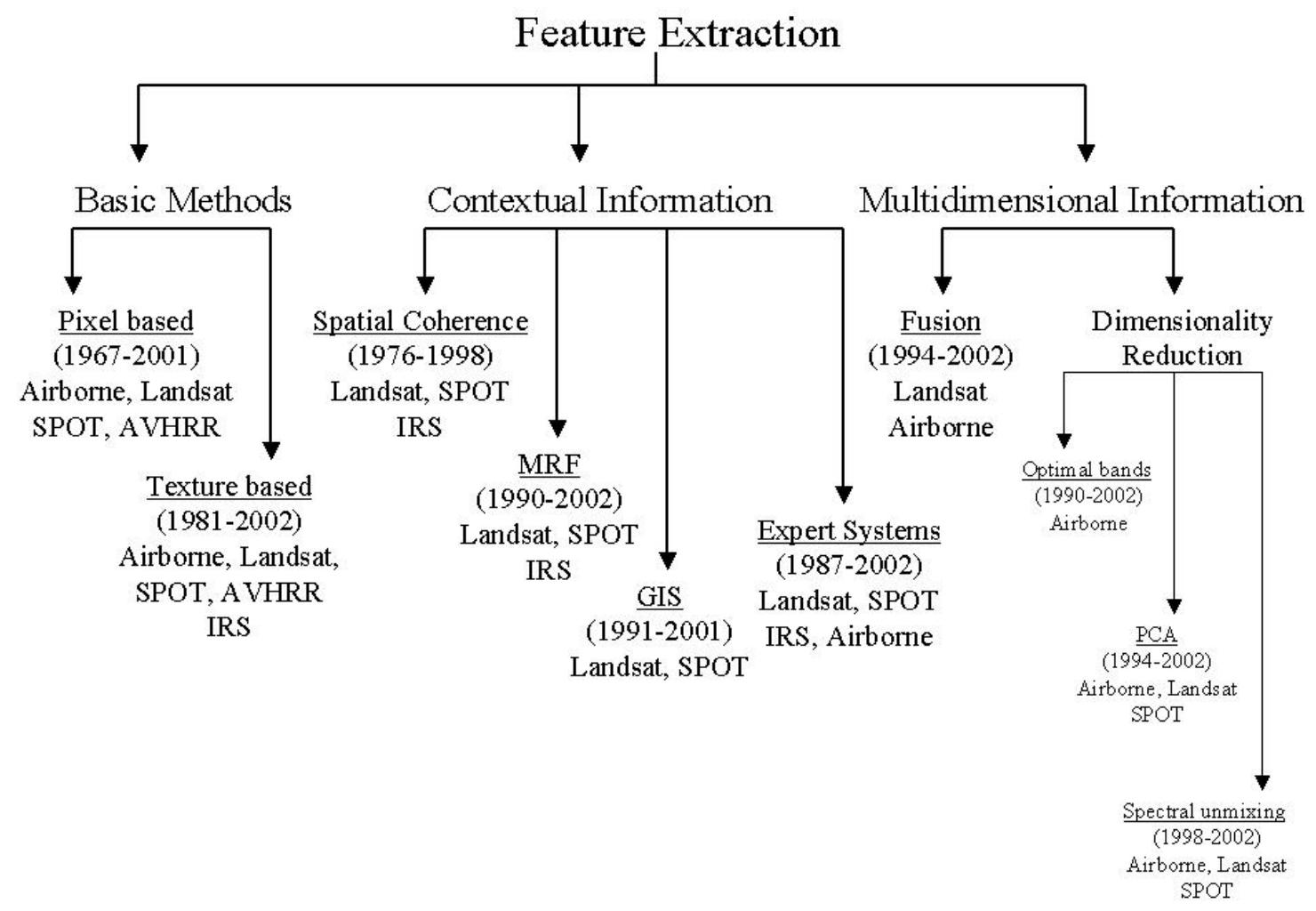

Figure 2.2: Taxonomy of the feature extraction methods 
In the following sections, we discuss these methods in depth. We also suggest future research directions based on these methods. Let's start with the basic feature extraction methods since they offered an initial approach to solve the land use problem.

\subsection{Basic Feature Extraction Methods}

We consider two basic methods of land use classification in this section. These are methods based on pixel and texture analysis for feature extraction. These methods represent the earliest approaches to the land use classification problem. In fact, pixel based classification was the first approach explored by researchers. Texture analysis, introduced later, is still used as a powerful feature extraction method. The main similarity between these methods is that most systems based on them are fast and simple compared to the approaches that followed. Unlike later developments, these two approaches do not use spatial information explicitly.

\subsubsection{Pixel Based Methods}

The term "pixel" has a different context in the remote sensing domain than in computer vision and image processing. It can represent a large area with diverse characteristics. For example AVHRR images capture a $1.21 \mathrm{~km}^{2}$ area in a single pixel. This area may contain many different objects; such as buildings, roads segments, and different soil types and covers. Although recent sensors have around 1 $m$ resolution, each pixel may still cover multiple objects or surfaces. For individual object recognition, this may cause problems because the sampling is effectively below the Nyquist limit; however, in land use classification the aim is to classify a region. Thus instead of separate objects, their cumulative (spectral) characteristic is deemed 
most important. Because a large region is summarized in each pixel, pixel-based methods assume that the gray level or multispectral response at a given pixel suffices for general (average) land use classification.

$\mathrm{Fu}$ et al. [2] considered agricultural data in remotely sensed images. Although studies on aerial and satellite images may have appeared prior to this paper, we take it as the first key paper trying to automate the process. Fu et al. used spectral values of pixel values directly as features. They had 12 bands (each taken as a separate feature) in the 0.4-1.0 $\mu \mathrm{m}$ spectral range. To obtain the most representative features, they applied feature selection methods. Finally, they used standard parametric and non-parametric statistical classifiers to obtain the class labels. They also mentioned the importance of spatial information, however they did not use it in their system.

As a first attempt to solve the land use classification problem, they pointed out all the important steps to be taken such as feature extraction, feature selection (data dimensionality reduction), classification, and the importance of spatial information. So, this work set the research direction to be followed for the future researchers.

Following Fu's seminal work, there were numerous other pixel based classification studies, for example [3-9] (Table 2.13).

To compare the performances of these studies directly is not scientifically sound because each uses a different image type and geographical location. But generally, pixel classification rates lie in the mid $80 \%$ to mid $90 \%$ range. The popularity of pixel based classification methods peaked in the 1980s when image resolution was insufficient for other approaches. Recent pixel based classification algorithms use additional information, setting them apart from the initial studies. 


\begin{tabular}{|c|l|l|l|c|}
\hline Year & Author & Image Type & Location & Performance \\
\hline 1967 & Fu et al. $[2]$ & Airborne & Indiana & $94.3 \%$ \\
\hline 1980 & Landgrebe [3] & Landsat & Indiana & $94.1 \%$ \\
\hline 1987 & Khorram et al. $[4]$ & Landsat & Carolina & $83.9 \%$ \\
\hline 1990 & Etchegorry [5] & Landsat, SPOT & Indonesia & - \\
\hline 1990 & Lloyd [6] & AVHRR & Earth & - \\
\hline 1994 & De Fries and Townshend [7] & AVHRR & New York & $86.8 \%$ \\
\hline 1996 & Hoffbeck and Landgrebe [8] & AVIRIS & Nevada & $96.4 \%$ \\
\hline 2001 & Bandyopadhyay et al. $[9]$ & SPOT & India & $88.9 \%$ \\
\hline
\end{tabular}

Table 2.13: Pixel based classification

\subsubsection{Texture Analysis Based Methods}

The next group of papers frame the problem in terms of texture analysis. Since satellite and aerial images contain many objects while the main objective is to classify regions, researchers have turned to texture analysis methods. Texture analysis has been studied in the computer vision literature for many years. Several "definitions" of texture have been formulated by researchers and there is no general consensus that can be formalized mathematically. Some definitions are perceptually motivated; others are driven completely by the application at hand. One early and influential definition is due to Haralick:

"The image texture we consider is non figurative and cellular... An image texture is described by the number and types of its (tonal) primitives and the spatial organization or layout of its (tonal) primitives... A fundamental characteristic of texture: It cannot be analyzed without a frame of reference of tonal primitive being stated or implied. For any smooth gray-tone surface, there exists a scale such that when the surface is examined, it has no texture. Then as resolution increases, it takes on a fine texture and then a coarse texture." [10]. 
For the interested reader, we recommend two excellent review papers by Tuceryan and Jain [11] and Wezska et al. [12]. In nearly all texture analysis methods in use for land use classification, an operation performed on an image region produces a representative number for use in classification.

Irons and Petersen [13] applied texture analysis methods to a Landsat image of Clearfield County, Pennsylvania, using the mean, variance, skewness, kurtosis, and the mean of the maximum gray level difference as features. They conclude that these first order statistical features are not very useful for land use classification. Although their results were not encouraging, applying texture analysis as a feature extraction method in land use classification was the main contribution of their paper.

In another study, Conners et al. [14] applied the Grey Level Cooccurrence Matrix (GLCM) features to a Sunnyvale, California image. GLCM features are extracted based on (estimated) conditional probabilities of neighboring grey level values $[15,16]$. These features are second order statistics. Being a powerful feature extraction method in texture analysis, GLCM has been applied extensively in land use classification. Conners et al. were the first to provide this method to solve the land use classification problem.

Many researchers have applied texture analysis methods to land use classification [17-25] (Table 2.14).

As Table 2.14 shows, the classification rates are in the $80 \%-90 \%$ range. These methods grew in popularity during the late 1990s as the resolution of satellite images increased and simple pixel based classification became obsolete. 


\begin{tabular}{|c|l|l|l|c|}
\hline Year & Author & Image Type & Location & Performance \\
\hline 1981 & Irons and Petersen [13] & Landsat & Pennsylvania & - \\
\hline 1984 & Conners et al. $[14]$ & Airborne & California & $83.4 \%$ \\
\hline 1990 & Marceau et al. $[17]$ & SPOT & Canada & $80.0 \%$ \\
\hline 1991 & Lee and Philpot [18] & Landsat & New York & $80.0 \%$ \\
\hline 1992 & Sali and Wolfson [19] & SPOT & New York & - \\
\hline 1995 & Baraldi and Parmiggiani $[20]$ & AVHRR & Antarctica & - \\
\hline 1999 & Smits and Annoni $[21]$ & IRS & Italy & $90.0 \%$ \\
\hline 2000 & Karathanassi et al. $[22]$ & SPOT & Greece & $89.6 \%$ \\
\hline 2001 & Shaban and Dikshit $[23]$ & SPOT & India, Egypt & $80.2 \%$ \\
\hline 2001 & Cihlar et al. $[24]$ & Landsat & Canada & - \\
\hline 2002 & Parinello and Vaughan $[25]$ & AVHRR & Scotland & - \\
\hline
\end{tabular}

Table 2.14: Texture analysis based classification

\subsection{Methods Using Contextual Information}

Another major category of research focuses on the use of contextual information in land use classification. We divided these studies into four subgroups: spatial coherence, Markov random fields, geographic information systems, and expert systems. These methods account for spatial interactions between neighboring pixels and regions in classification to improve performance.

\subsubsection{Spatial Coherence}

In satellite and aerial images similar land types tend to be located nearby. In other words, neighboring pixels have high probability of belonging to the same land class unless they lie on a border between two different classes. This observation led researchers to imbed spatial information into land use classification.

When seen from overhead, similar land types tend to be more or less contiguous that is neighboring pixels have a high probability of belonging to the same land 
class; pixels lying on a border between two different classes are relatively rare. This observation has led researchers to exploit spatial coherence in land use classification. Kettig and Landgrebe [26] were among the first researchers to use this constraint. They grouped contiguous pixels into homogenous sets and then classified the groups. They applied their algorithm to two Landsat and two aircraft images and observed an improvement in the classification performance. Their paper provides as a key contribution in the use of spatial coherence.

Baraldi and Parmiggiani [27] used a SPOT image from Modena, Italy to test a classifier for urban areas, using textural features and a region growing algorithm as the contextual classifier. They concluded that the contextual classifier was performing as desired, but that textural features are not satisfactory for SPOT imagery. Using region growing as a grouping method for land use classification was the unique feature of this paper.

Jensen [28] used the contextual classification idea with the GLCM features. In this way he combined both methods. He tested on a 30 meter resolution Landsat image of Bangkok and gave a visual interpretation. Unfortunately, this interpretation is inconclusive for assessing the power of the method. However, combining the GLCM features with contextual classification was the extension, this paper offered.

Richards et al. [29] applied probabilistic relaxation to inject spatial information into the classification process. Probabilistic relaxation is a labelling method taking into account interactions between neighboring pixels [30]. Richards et al. first obtained the Maximum Likelihood (ML) classification labelling and applied relaxation to these labels to include spatial coherence. Using relaxation (which proved its power 
in a wide range of applications) instead of other spatial information processing systems was the key contribution of this paper.

Other work in this area includes [31-46] (Table 2.15).

\begin{tabular}{|c|l|l|l|c|}
\hline Year & Author & Image Type & Location & Performance \\
\hline 1976 & Kettig and Landgrebe [26] & Landsat & - & - \\
\hline 1980 & Thomas [31] & Landsat & New Zealand & - \\
\hline 1980 & Fu and Yu [32] & Landsat & Indiana & $89.2 \%$ \\
\hline 1982 & Tilton et al. $[33]$ & Landsat & Kansas & $86.0 \%$ \\
\hline 1982 & Richards et al. $[29]$ & - & - & $81.0 \%$ \\
\hline 1982 & Wharton [34] & Simulation & - & $88.0 \%$ \\
\hline 1983 & Gurney and Townshend [35] & Landsat & UK & $70.0 \%$ \\
\hline 1987 & Cushnie [36] & - & - & - \\
\hline 1990 & Khazenie and Crawford [37] & Simulation & - & $87.3 \%$ \\
\hline 1990 & Jensen [28] & Landsat & Bangkok & - \\
\hline 1990 & Kusaka et al. $[38]$ & SPOT & Japan & $78.0 \%$ \\
\hline 1990 & Baraldi and Parmiggiani $[27]$ & SPOT & Italy & - \\
\hline 1991 & Kim and Crawford [39] & SPOT & Texas & - \\
\hline 1991 & Alonso and Soria [40] & Landsat & Spain & $70.4 \%$ \\
\hline 1992 & Gong and Howarth [41] & SPOT & Canada & $61.6 \%$ \\
\hline 1992 & Gong and Howarth [42] & SPOT & Canada & $72.2 \%$ \\
\hline 1996 & Barnsley and Barr [43] & SPOT & England & $97.1 \%$ \\
\hline 1997 & Lobo [44] & Landsat & Spain & $84.5 \%$ \\
\hline 1998 & Kertikeyan et al. $[45]$ & IRS & - & $91.0 \%$ \\
\hline 1998 & Sharma and Sarkar [46] & Landsat & India & $89.5 \%$ \\
\hline
\end{tabular}

Table 2.15: Spatial information based classification

As Table 2.15 shows, the classification rate for these methods lie in the range of $70 \%$ to $97 \%$. Although most popular in the mid-1990s, they survive in different forms, as we mention next. 


\subsubsection{Markov Random Fields}

Another popular tool to imbed contextual information into classification is the Markov Random Field (MRF). This method models the joint statistics of a small neighborhood of pixels. Its popularity for texture analysis initiated its application to land use classification. The seminal work of Geman and Geman [47] provides an excellent development of this method. We consider MRFs to be part of the continuum of spatial information based methods.

The method can be briefly summarized as follows. The brightness level at an image point is highly dependent on (and therefore correlated with) the brightness level of neighboring points, assuming the image is of a real coherent scene and not merely random noise. The MRF provides a precise model for this dependence.

Zhang et al. [48] introduced the MRF as a contextual classification algorithm. They captured the correlation between neighboring pixels by MRF models then applied stochastic relaxation to obtain the final class labels. They applied this method on subwindows of test images with four spectral bands. Zhang's work showed the usefulness of MRF modelling in capturing the context information for land use classification problem. Different from the spatial information, this method used stochastic modelling via MRF.

Other researchers also used MRFs in their systems [49-56] (Table 2.16).

As Table 2.16 shows, the classification performance for the MRF-based systems ranges from $77 \%$ to $97 \%$. Publication dates indicate that MRF based methods gained acceptance in the late 1990s. 


\begin{tabular}{|c|l|l|l|c|}
\hline Year & Author & Image Type & Location & Performance \\
\hline 1990 & Zhang et al. [48] & Landsat & Virginia & $77.0 \%$ \\
\hline 1992 & Jeon and Landgrebe [49] & Landsat & Indiana & $82.8 \%$ \\
\hline 1996 & Jhung and Swain [50] & Landsat & Indiana & $86.2 \%$ \\
\hline 1996 & Solberg et al. $[51]$ & Landsat & Norway & $96.9 \%$ \\
\hline 1999 & Yamazaki and Gingras [52] & Landsat & Canada & $78.2 \%$ \\
\hline 1999 & Tso and Mather [53] & Landsat & Sudan & $81.0 \%$ \\
\hline 1999 & Kerfoot and Bresler [54] & Landsat & Illinois & - \\
\hline 1999 & Yu et al. $[55]$ & SPOT & France & - \\
\hline 2002 & Sarkar et al. $[56]$ & IRS & - & $95.0 \%$ \\
\hline
\end{tabular}

Table 2.16: MRF based classification

\subsubsection{Geographical Information Systems}

Another class of methods to exploit contextual information are those using Geographical Information Systems (GIS) and topological maps in classification. In this case, the contextual information derives from prior knowledge of a specific region, rather than generic observations regarding spatial coherence in land use. As such, these systems are geared to land use updating rather than raw classification.

One of the first applications of this idea is by Pedley and Curran [57]. They grouped pixels according to the GIS data and classified these groups. Compared to pixel classification, Pedley and Curran obtained improved performance. Since recently GIS has been used extensively, Pedley and Curran's work can be taken one of the most effective tools in updating land use classification. However, their method requires a prior classification which limits its application.

The work in this area includes [58-61] (Table 2.17).

As Table 2.17 shows, the classification performance ranges from $62 \%$ to $92 \%$ for these methods. Although researchers started using these methods around 1991 they 


\begin{tabular}{|c|l|l|l|c|}
\hline Year & Author & Image Type & Location & Performance \\
\hline 1991 & Pedley and Curran [57] & SPOT & - & $62.1 \%$ \\
\hline 1993 & Wang [58] & Landsat & Canada & $83.8 \%$ \\
\hline 2000 & De Bruin and Gorte [59] & Landsat & Spain & $79.5 \%$ \\
\hline 2000 & De Bruin [60] & Landsat & Spain & - \\
\hline 2001 & Baban and Yusof [61] & Landsat & Malaysia & $92.0 \%$ \\
\hline
\end{tabular}

Table 2.17: GIS classifier improvements

are becoming popular nowadays as GIS databases become more prevalent and populated. Indeed, we expect most future systems - using all available prior contextual information and spatial coherence - to incorporate such databases, as well.

\subsubsection{Expert Systems}

Expert systems cover a large range of methods such as artificial intelligence, rule based systems, knowledge based representations, Bayesian belief networks, and case based reasoning. Jackson's text [62] represents a good introductory reference on this subject.

There have been numerous studies in land use classification using expert systems in which the rule base encapsulates the contextual information. To our knowledge, the initial work of Wharton [63] in 1987 was the first study to use expert systems in land use classification (but not in remote sensing).

He considered spectral values of pixels (as in previous studies) as features. He then used a knowledge based approach to capture the spectral relationships between classes. To assign the final class labels, he iteratively updated class labels based on the knowledge base till the class labels stabilize (till most of the pixels satisfy the neighborhood rules). 
Wharton's approach was another perspective for contextual information processing. Unlike previous three approaches, he captured the context information by defining rules on the neighborhood regions. This way, his approach takes the perspective of artificial intelligence.

Ton et al. [64] also used a complex knowledge-based system to classify Landsat images. They extracted major land types using a hierarchical classifier, followed by a rule-based clustering algorithm to obtain fine classification. They obtained promising results, albeit with a limited test set. A rule based clustering algorithm is also another powerful approach to obtain the final class labels.

Other research in this area includes [65-70] (Table 2.18).

\begin{tabular}{|c|l|l|l|c|}
\hline Year & Author & Image Type & Location & Performance \\
\hline 1987 & Wharton [63] & Landsat & Maryland & $85.0 \%$ \\
\hline 1991 & Ton et al. $[64]$ & Landsat & Michigan & $86.0 \%$ \\
\hline 1995 & Kartikeyan et al. $[65]$ & IRS & - & $92.0 \%$ \\
\hline 1997 & Jensen [66] & Landsat, SPOT & Ghana & - \\
\hline 2000 & Casasnovas [67] & Landsat & Spain & $81.4 \%$ \\
\hline 2000 & Barr and Barnsley [68] & Daedalus & UK & $82.4 \%$ \\
\hline 2001 & Stefanov et al. $[69]$ & Landsat & Arizona & $85.0 \%$ \\
\hline 2002 & Datcu et al. $[70]$ & Daedalus & UK, Germany & $88.8 \%$ \\
\hline
\end{tabular}

Table 2.18: Expert systems

Table 2.18 indicates the increasing popularity of these systems with their performance ranging from $81 \%$ to $92 \%$. 


\subsection{Methods Summarizing Multidimensional Information}

The following papers focus on the dimensionality of the data to help (or replace) feature extraction and the inclusion of spatial information. Either by reducing the dimensionality of the data or by fusing different representations of the same pixel, they aim to improve performance. We divide these papers into two subgroups: data dimensionality reduction and data-decision fusion.

\subsubsection{Data Dimensionality Reduction}

In land use classification, the same scene is often imaged at multiple wavelengths (bands). For multispectral images this representation is around four to seven bands; hyperspectral images may go up to 240 bands (dimensions). Therefore, each pixel in these images is represented as a multidimensional vector, having four to 240 en-

tries. Not all of these bands are equally useful for classification, depending on the application. Therefore the data dimension may be reduced either by selecting useful bands (optimal band selection) or by transforming the original space to one of lower dimension (principal components analysis and spectral unmixing).

\section{Optimal Band Selection}

Mausel et al. [71] compared four optimum band selection methods for land use classification. Their paper serves as a good reference for researchers planning to use these band selection techniques. Unlike dimension reduction by transforming the original space, these methods serve as feature selection criteria in the original space. Tsai and Philpot's [72] paper is another work on optimal band selection (Table 2.19). 


\begin{tabular}{|c|l|l|l|c|}
\hline Year & Author & Image Type & Geographic location & Performance \\
\hline 1990 & Mausel et al. $[71]$ & Airborne & Texas & $92.2 \%$ \\
\hline 2002 & Tsai and Philpot [72] & AVIRIS & California & $69.0 \%$ \\
\hline
\end{tabular}

Table 2.19: Optimal band selection

\section{Principal Components Analysis}

Principal Components Analysis (PCA) performs a linear transformation on a set of (possibly) correlated random vectors to represent them in a new space such that they are uncorrelated. The new coordinate space is related to the original by rotation about the origin, such that the new coordinate axes are aligned with the dominant directions of dispersion (the principal components) of the data, as inferred from its covariance matrix. For a thorough treatment, see [73].

Harsyani and Chang [74] considered an extended version of the PCA to reduce data dimensionality. As we recall, in hyperspectral images a pixel may be represented by (up to) a 240 dimensional vector. For such high dimensional data, PCA and similar transformations are needed for reliable classification. Harsyani and Chang aimed to reduce the data dimensionality while retaining most of the discriminating information via their method. This paper pointed to another powerful research area for data dimension reduction.

Similar studies on PCA and related transformation methods include [75-78] (Table $2.20)$. 


\begin{tabular}{|c|l|l|l|c|}
\hline Year & Author & Image Type & Geographic location & Performance \\
\hline 1994 & Harsyani et al. $[74]$ & AVIRIS & Nevada & - \\
\hline 1999 & Jia and Richards [75] & AVIRIS & California & $97.8 \%$ \\
\hline 2000 & Kuplich et al. [76] & Landsat & Brazil & $94.8 \%$ \\
\hline 2000 & Ren and Chang [77] & Landsat & Virginia & - \\
\hline 2001 & Kumar et al. $[78]$ & AVIRIS & Florida & $95.0 \%$ \\
\hline
\end{tabular}

Table 2.20: PCA

\section{Spectral Unmixing}

To overcome problems in PCA, many researchers considered spectral unmixing techniques. Chang et al. [79] define spectral unmixing as:

" Linear spectral unmixing is widely used approach in multispectral/hyperspectral imagery to determine and quantify individual spectral signatures in a mixed pixel."

Chang et. al. also showed the usefulness of spectral unmixing in land use classification. Papers using spectral unmixing methods include [80-85] (Table 2.21).

\begin{tabular}{|c|l|l|l|c|}
\hline Year & Author & Image Type & Geographic location & Performance \\
\hline 1998 & Chang et al. $[79]$ & AVIRIS & Nevada & - \\
\hline 1999 & Chang et al. [80] & SPOT & USA & - \\
\hline 2000 & Chang and Ren [81] & HYDICE & Maryland & $95.0 \%$ \\
\hline 2001 & Small [82] & Landsat & New York & - \\
\hline 2001 & Roessner et al. $[83]$ & DAIS 7915 & Germany & $80.0 \%$ \\
\hline 2002 & Chang [84] & AVIRIS & Nevada & - \\
\hline 2002 & Verhoeye et al. $[85]$ & SPOT & Nigeria & $91.0 \%$ \\
\hline
\end{tabular}

Table 2.21: Spectral unmixing 
As we consider Tables 2.19, 2.20, and 2.21, we notice that data dimension reduction has a positive effect on classification performance. The publication dates, suggest that these methods are growing more popular.

\subsubsection{Data and Decision Fusion}

In decision fusion, decisions of different classifiers are combined in such a way that the final classification performance is improved. There have been many methods proposed for this purpose and an excellent review paper on this subject is by Kittler et. al. [86].

Instead of data dimension reduction, all of the bands are used in decision fusion. However, classification is applied to each band separately, and the assigned class labels are fused. In a way this is the dual of data dimension reduction, in that the order of dimension reduction and classification is replaced.

Jeon and Landgrebe [87] proposed two decision fusion based classifiers. With their method they reported a $5 \%$ improvement in performance. This improvement itself indicates the usefulness of the idea.

Decision fusion have been studied by many authors in land use classification [8892] (Table 2.22).

In a related study, Solberg et al. [93] applied data fusion on pixel basis and obtained superior performance. The goal of data fusion is to obtain a useful representation from the multidimensional data set. Clark and Yuille's book [94] is a very good reference on this subject.

As Table 2.22 shows, these methods have been considered since 1994 and most studies are quite new (by looking at their publication dates). 


\begin{tabular}{|c|l|l|l|c|}
\hline Year & Author & Image Type & Geographic location & Performance \\
\hline 1994 & Solberg et al. $[93]$ & Landsat & Norway & $95.0 \%$ \\
\hline 1997 & Benediktsson et al. $[88]$ & Landsat & Colorado & $75.0 \%$ \\
\hline 1999 & Jeon and Landgrebe [87] & Landsat & Indiana & $83.6 \%$ \\
\hline 1999 & Bennediktsson et al. $[89]$ & Landsat & Portugal & $86.0 \%$ \\
\hline 1999 & Jimenez et al. $[90]$ & AVIRIS & Indiana & $96.0 \%$ \\
\hline 2000 & Steele [91] & Landsat & Idaho & $68.0 \%$ \\
\hline 2002 & Smits [92] & Landsat & Netherlands & $95.0 \%$ \\
\hline
\end{tabular}

Table 2.22: Decision fusion

\subsubsection{Summary of the Methods}

In the previous sections, we grouped studies in land use classification to three main categories. Initial research in the area started with basic methods $[2,14]$. The next class of studies dealt with contextual information $[48,63]$. The last group of studies dealt with data dimension reduction and decision fusion $[74,79,87]$. These key papers summarize the main trends in land use classification since 1967. Next, we plot the number of papers published in three year intervals from 1967 to 2002 in Fig. 2.3 to summarize the popularity of these methods.

Fig. 2.3 points to the following conclusions. After their introduction, methods on contextual information dominated basic methods. This indicates the importance of contextual information in land use classification. Although introduced later, work on multidimensional information has recently taken the lead in the number of papers published. This indicates that the main trend nowadays is towards multidimensional information processing. The next most promising direction is in contextual information processing. If we consider these main groups in detail, we observe the following. 


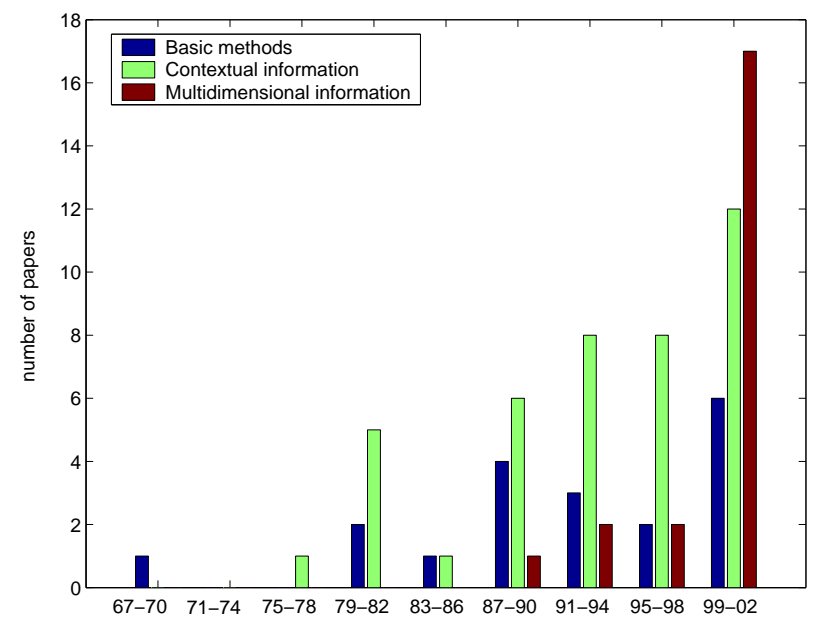

Figure 2.3: Number of papers published in three year time intervals from 1967 to 2002

Within basic methods, texture analysis is becoming more popular than pixel based techniques. If we check the usage of sensors, almost all of them have been used for basic methods category. One important point is that AVHRR finds use in basic methods only. Its limited resolution renders it unsuitable for the more sophisticated techniques that have followed.

Methods using contextual information can be sorted based on their recent publication dates into: expert systems, GIS, MRF, and spatial coherence. In this category expert systems are becoming more popular. As we mentioned, GIS based systems are likely to take the lead from expert systems in the near future. Sensors used in this category are dominantly Landsat and SPOT. The properties of these sensors seem suitable for capturing the context. 
We can sort the methods summarizing the multidimensional information by their recent publication dates into: spectral unmixing, PCA, and data and decision fusion. Spectral unmixing, proposed to overcome the shortcomings of PCA is popular nowadays, and PCA based data dimension reduction techniques follow. Sensors used in this category are dominantly airborne for data dimension reduction and Landsat for decision fusion. Since data dimension reduction techniques are proposed for high dimensional data, it is self-evident that they find use with airborne sensors producing hyperspectral data. As for decision fusion, Landsat seems to be the most suitable sensor based on the current publications in this area.

\subsection{Summary of the Chapter}

In this chapter, we summarized the main trends in land use classification by reviewing a big and influential portion of the literature. We also summarized the characteristics of remote sensing sensors, both satellite based and airborne. Since we reviewed papers published in a long period of time, we also included the historical evolution of those sensors. Next, we will introduce our land use classification measures on panchromatic images. 


\section{CHAPTER 3}

\section{Land Classification}

After a through survey on land use classification, we begin with a set of measures based on straight lines. Subsequent detailed analyses (counting and classifying dwellings, for example) can then be confined to developed areas. Straight line structures will be more prevalent and more organized in developed areas than in wilderness or rural areas. Our objective at this stage is the (rough) classification of the image into regions of little or no development, suburban regions likely to be residential, and urban areas.

Our statistical measures in this chapter are based on the orientation, length, contrast, periodicity, and location of straight lines. We applied Bayes, Parzen window, and nearest neighbor $(\mathrm{NN})$ classifiers to label each image region. Initially, we defined a two-class problem to discriminate "urban" and "not urban" regions and obtained excellent results (roughly $87 \%$ correct classification).

Next, we addressed the far more difficult problem of discriminating among urban, residential (suburban), and wilderness areas in a three class problem. The direct approach was less successful in this case, largely because suburban regions bridge the other two in our feature space much as they do on the ground. Therefore; in an attempt to extract suburban regions, we introduced an enhancement based on 
the principles of perceptual organization. Perceptual organization is that process, or set of processes, by which a vision system (natural or artificial), organizes detected features in images based on various Gestaltic clues [95]. Perceptual organization is therefore, the ability to impose structural regularity on sensory data, grouping sensory primitives having a common underlying cause. We introduced a spatial coherence constraint and performed grouping in the feature space. Via this novel perceptual grouping approach, the results improved significantly.

Although there has been extensive work on land use classification, no structural approaches to this problem have been reported. Our approach, being totally based on straight lines, offers the first such solution, to our knowledge. This approach shows very promising results in extensive testing over a wide variety of land development patterns. Besides the structural approach to land classification, our new spatial coherence method, based on perceptual organization principles, also offers very promising results by combining the feature and image spaces.

\subsection{Line Support Regions (LSR) and Straight Line Extrac- tion}

Burns et al. [96] introduced the concept of line support regions, constructed by grouping contiguous pixels of consistent gradient orientation. His gradient calculation used simple $2 \times 2$ masks, which creates many false alarms in our high resolution data. Therefore, we turned to the scale-controllable IIR edge detection filter proposed by Sarkar and Boyer [97] to compute an optimally smoothed gradient. Then, instead of marking edges in the usual way, we organized the image into line support regions in a manner reminiscent of Burns' work. 
We calculated the scale-controllable gradients for the $x$ and $y$ components, $G_{x}$ and $G_{y}$, by convolving the image in the $x$ and $y$ directions with the Sarkar-Boyer filter. This is achieved as follows. Two non-causal filters, the projection filter $h(n)$ and the edge detection filter $e(n)$, are successively applied to rows and columns of the image. Impulse responses for these filters for $n>0$ are given in Eqns. 3.1 and 3.2:

$$
\begin{gathered}
h(n)=-e^{-\alpha n} \cos (\beta \alpha n+\pi / 2) \\
e(n)=\frac{e^{-\alpha n}}{\alpha}\left[-\frac{\cos (\beta \alpha n+\pi / 2)-\beta \sin (\alpha \beta n+\pi / 2)}{1+\beta^{2}}\right]
\end{gathered}
$$

where $n$ is either $x$ or $y$, depending on orientation. The derivation of these impulse responses is beyond the scope of this chapter; see [97].

The Projection filter smoothes the image to lower the noise. The edge detection filter is designed to respond optimally to step edges in the image. In effect, two (separable) 2D impulse responses are created. The first consists of the projection filter in the $y$ direction and the detection filter in $x$; the second is orthogonal to the first. The projection filter is the integral of the edge detection filter, which greatly simplifies implementation. We used the IIR implementation of these filters as suggested by Sarkar and Boyer.

After extensive testing, we selected a fairly small scale parameter $(\alpha=1)$, to resolve buildings in close proximity. The scale of the filter is directly related to the resolution of the image. Since we are working on satellite images, the scale parameter should be adjusted in accordance with the size of ground features of interest (in pixels) and their spacing. A small scale parameter resolves small and closely located features. 
On the other hand, a large scale parameter captures general characteristics of the image. This adjustment capability (absent in Burns' implementation) allows users to apply this method to a wide variety of image resolutions. Therefore, our system responds to changes to this parameter. Similarly, we set $\beta=70$ for implementation. Our system is less sensitive to this parameter, which controls a tradeoff among localization, signal-to-noise ratio out of the filter, and spurious responses (see [97] for details). The impulse responses of projection and edge detection filters with these parameters are given in Fig. 3.1.

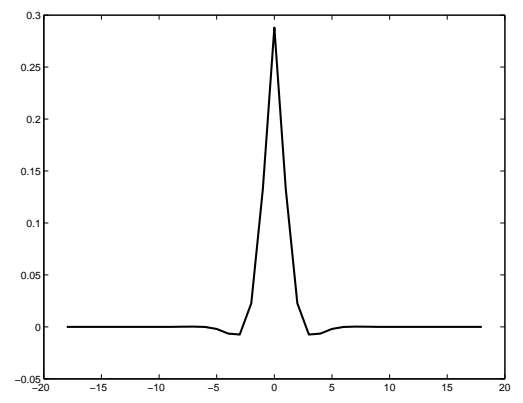

(a) Projection filter

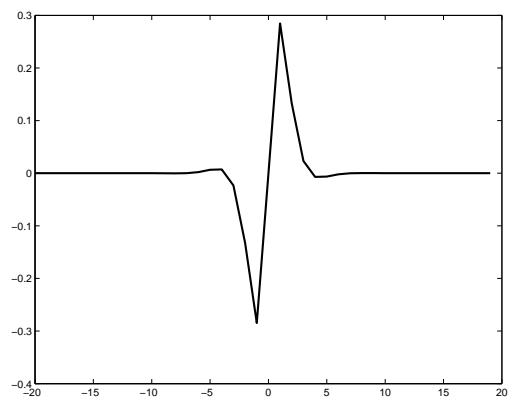

(b) Edge detection filter

Figure 3.1: Impulse responses for the projection and edge detection filters

Let $f(x, y)$ be the image and let $G_{x}(x, y)=\{e(x) h(y)\} * f(x, y)$ be the $x$ component of the smoothed gradient, computed by convolving the image with the projection filter (in $y$ ) and the detection filter (in $x$ ). Similarly, let $G_{y}(x, y)=\{e(y) h(x)\} * f(x, y)$ be the $y$ component. Then, the smoothed gradient direction at $(x, y)$ is:

$$
G(x, y)=\arctan \frac{G_{x}(x, y)}{G_{y}(x, y)}
$$


We discard pixels having gradient magnitude smaller than a given threshold (10 here) to suppress noise. Following Burns et al., we then used two quantizers and a voting method to obtain longer and more consistent line support regions. Each quantizer has eight bins of angular resolution $\pi / 4$ radians, the second one rotated by $\pi / 8$ radians. Two quantizers with shifted bin centers can accommodate candidate line support regions that would be broken in a single quantizer due to minor perturbations.

Burns et al. extracted the lines by intersecting a horizontal plane with the best fit plane to the local image surface. Following Tan [98] we improve on this approach by fitting an ellipse to each line support region perimeter using a Fourier descriptor approximation. The long axis of the ellipse (which requires only the first order descriptors) defines the straight line. This method is faster and more robust than plane fitting. We obtain the best fitting ellipse as follows. A complex periodic function, $u(k)=u(k+r T)$, for any integer values of $k$ and $r$ represents the outer boundary of the line support region. $T$ is the total number of points in the contour. Thus, the complex periodic contour can be approximated by a Fourier series as [99]:

$$
\hat{u}(k)=\sum_{n=0}^{T-1} U_{n} e^{j \frac{2 \pi n k}{T}}
$$

where $j=\sqrt{-1}$ and

$$
U_{n}=\frac{1}{T} \sum_{k=0}^{T-1} u(k) e^{-j \frac{2 \pi n k}{T}}
$$

If we insert the complex representation $u(k)=x(k)+j y(k)$ into Eqn. 3.5

$$
U_{n}=\frac{1}{T} \sum_{k=0}^{T-1} x(k) e^{-j \frac{2 \pi n k}{T}}+j \frac{1}{T} \sum_{k=0}^{T-1} y(k) e^{-j \frac{2 \pi n k}{T}}
$$

Applying Euler's formula, we get 


$$
\begin{gathered}
U_{n}=\frac{1}{T} \sum_{k=0}^{T-1} x(k)\left[\cos \left(\frac{2 \pi n k}{T}\right)-j \sin \left(\frac{2 \pi n k}{T}\right)\right] \\
+j \frac{1}{T} \sum_{k=0}^{T-1} y(k)\left[\cos \left(\frac{2 \pi n k}{T}\right)-j \sin \left(\frac{2 \pi n k}{T}\right)\right] \\
U_{n}=\alpha_{n}+j \beta_{n}
\end{gathered}
$$

with:

$$
\begin{aligned}
& \alpha_{n}=\frac{1}{T}\left[\sum_{k=0}^{T-1} x(k) \cos \left(\frac{2 \pi n k}{T}\right)+\sum_{k=0}^{T-1} y(k) \sin \left(\frac{2 \pi n k}{T}\right)\right] \\
& \beta_{n}=\frac{1}{T}\left[\sum_{k=0}^{T-1} y(k) \cos \left(\frac{2 \pi n k}{T}\right)-\sum_{k=0}^{T-1} x(k) \sin \left(\frac{2 \pi n k}{T}\right)\right]
\end{aligned}
$$

For our statistical features, defined below, we need the center of mass $\mu_{x y}$, the length $l$, and the orientation $\theta$ of each straight line. Fortunately, from just three Fourier coefficients, $\left(\alpha_{-1}, \beta_{-1}\right),\left(\alpha_{0}, \beta_{0}\right)$ and $\left(\alpha_{1}, \beta_{1}\right)$, we can obtain these quantities from Eqns. 3.11, 3.12, and 3.13 respectively.

$$
\begin{gathered}
\mu_{x y}=\left(\alpha_{0}, \beta_{0}\right) \\
l=2\left[\sqrt{\alpha_{1}^{2}+\beta_{1}^{2}}+\sqrt{\alpha_{-1}^{2}+\beta_{-1}^{2}}\right] \\
\theta=\frac{\arctan \left(\beta_{1} / \alpha_{1}\right)+\arctan \left(\beta_{-1} / \alpha_{-1}\right)}{2}
\end{gathered}
$$

Three sample images and the lines extracted from them for rural, residential, and urban areas are given in Fig. 3.2. The relative degree of organization over the three images is evident in their respective line structures. 


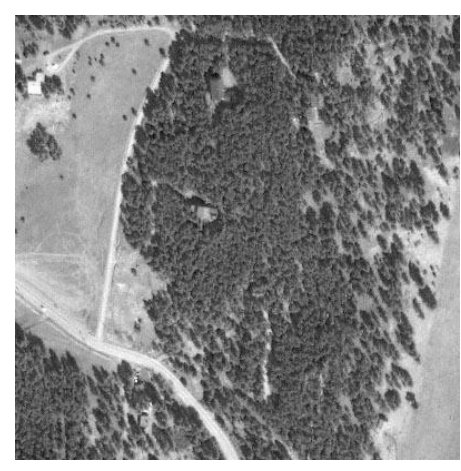

(a) The rural region

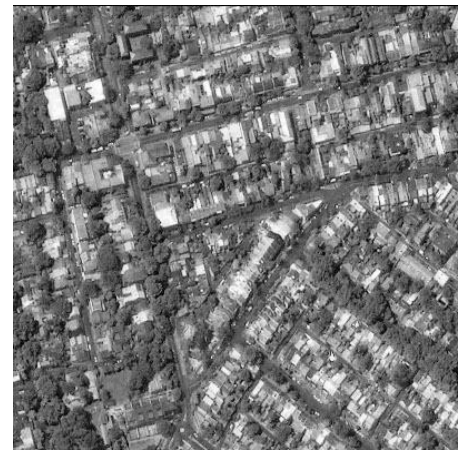

(c) The residential region

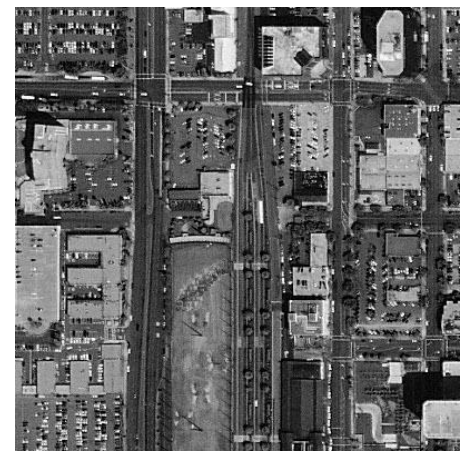

(e) The urban region

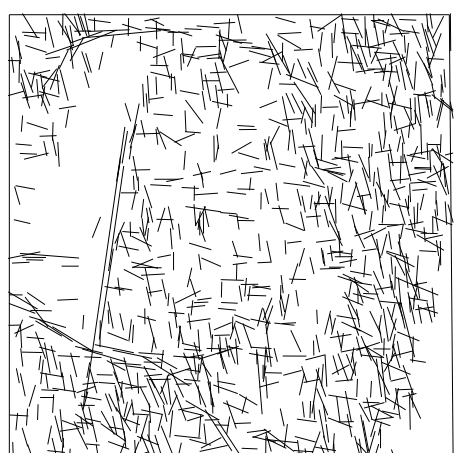

(b) Lines from the rural region

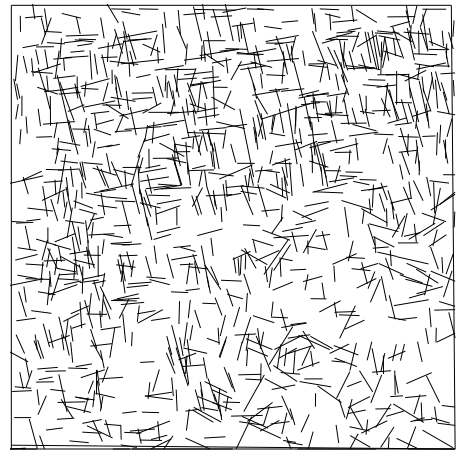

(d) Lines from the residential region

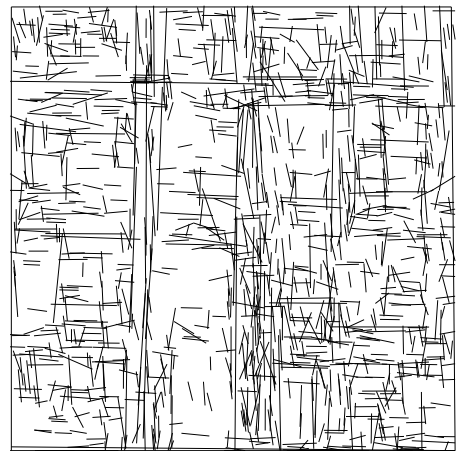

(f) Lines from the urban region

Figure 3.2: Sample subwindows and lines extracted from them 


\subsection{Statistical Feature Extraction}

We extract statistical features over $400 \times 400$ pixel windows, with $50 \%$ overlap in each direction. This degree of overlap represents a compromise in the interest of computational effort. Therefore, an organization lying partly in one window will likely lie totally inside a neighboring window. To evaluate performances of the features, we extracted the ground truths accordingly. However, if a final system is to be designed on this overlapping scheme, there are several possibilities to assign the final label to a window. As we apply the overlapping scheme, each pixel will have at most three labels assigned to it. One method to assign the final label would be to apply a voting scheme and use the majority. Another method would be more conservative approach; any pixel receiving at least one label of "urban" would be classified as such to minimize the probability of miss. These methods will be considered in a future study.

If a line extends beyond the window, that part is truncated and does not enter the calculations for this window. We discard straight lines shorter than five pixels (5 meters); such objects can not be approximated robustly due to low signal to noise ratios in the contour approximation and certainly do not offer strong evidence for "straightness".

Before defining the statistical measures, we give two definitions that we will use repeatedly. The first is the sample mean $\mu$; we assume this is self-explanatory. The second is the entropy $E$ of an approximate probability mass function (pmf). Let the approximate pmf be given as $h(i)$ for $i=1, \ldots, N$. The corresponding entropy is: 


$$
E=-\sum_{i=1}^{N}\left[h(i) \log _{2}(h(i))\right]
$$

The entropy measures the amount of information obtained, on average, in drawing samples from a given distribution. In effect, this captures the uncertainty, or randomness, in the experiment.

For each feature we extract, we obtain an approximate probability mass function from which we compute the sample mean and the entropy. We set the histogram bin size for each feature experimentally; they are not especially critical.

In this study we considered seven statistical features. The most promising of these were:

- Average line length, $\mu_{l}$

- Entropy of line length, $E_{l}$

- Average line contrast, $\mu_{c}$

- Entropy of line contrast, $E_{c}$

For completeness, we summarize the others in the Appendix 3.6.

\subsubsection{Length}

The dominant shape of the buildings and street segments in urban regions is rectangular (or compositions of rectangles). This assumption is valid only locally (such as in $400 \times 400$ windows) for most cities but this suffices because we calculate our measures over local regions. In wilderness (or rural) regions, due to the absence of human activity, we expect shorter and more randomly distributed straight lines. 
This observation motivates two statistical features to grade land development by the length of straight lines. The first is the sample mean of the length of straight lines, $\mu_{l}$, in the given image window.

The second feature measures the entropy in the distribution of line lengths, $E_{l}$, in the given image window. According to our hypothesis, not only will image features be shorter in undeveloped areas, they should also be more randomly distributed in length. Therefore, the distribution of the lengths in a given window can also provide useful information for grading land development and we capture the randomness in the entropy. We obtain the entropy of the length distribution as follows. We form a histogram of 37 bins (with binwidth $=4$ pixels), where the first bin is centered at 5 pixels and the last at 150 pixels. The maximum bin value represents the longest line segment such that the corresponding bin has significant probability and was obtained experimentally. The binwidth is picked to obtain a fairly smooth histogram. These values are not critical in feature extraction. Therefore, our system has a robust characteristics to their small variations.

The histogram is normalized to approximate a probability mass function. Let the normalized vote for bin $i$ be $h_{l}(i)$ for $i=1, \ldots, 37$. The entropy of line length is then obtained from Eqn. 3.14.

\subsubsection{Contrast}

Contrast provides another indicator of the level of development in a given area. In urban regions for example, we encounter greater variation in the color of buildings, sharp dark-light transitions between asphalt roads and shiny roofs, and the juxtaposition of buildings and vegetation. Such high contrast variations are less likely in 
wilderness (or rural) regions. This observation let's us to define two features based on contrast.

We define the contrast for each line support region from the gradient calculations as follows. Let pixels in a given line support region form a set $L S ;(x, y) \in L S$ represents a pixel in that line support region. The contrast value for the line support region, clsr is the maximum directional derivative magnitude over the region as given in Eqn. 3.15:

$$
c l s r=\max _{(x, y) \in L S}\left[\max _{d \in\{x, y\}}\left|G_{d}(x, y)\right|\right]
$$

Our tests of the true directional derivative magnitude produced similar classification performances; for simplicity we confine our attention to the $x$ and $y$ directions.

We also tested the average directional derivative magnitude, but the classification results based on this definition were not encouraging because in averaging, low contrast values dominate. The maximum directional derivative definition is biased towards high contrast values, which helps in classification. We obtain our first contrastbased feature, the sample mean $\mu_{c}$, from clsr values.

Our second feature is the line contrast entropy $E_{c}$. To compute it, we quantize the contrast range to 31 bins (with binwidth $=95$ ), where the first bin is centered at 5 and the last bin at 3000. These values are obtained experimentally as in length histograms, and found not to be critical. To build the histogram, each line support region votes with a weight proportional to the length of the line it represents. We then normalize the histogram as usual. Let the normalized vote for bin $i$ be $h_{c}(i)$ for $i=1, \ldots, 31$. Then the contrast entropy is obtained from Eqn. 3.14. 


\subsection{Feature Based Grouping for Suburbia Detection}

Levels of development in neighboring areas are not statistically independent. Cities tend to grow from a core outwards; apart from the intervention of natural barriers such as water and mountains, and land development expands more or less coherently. These observations indicate the importance of spatial information in classification. Kettig and Landgrebe [26] (among others) added spatial information for land cover classification.

To recover suburban regions, we have developed a grouping and classification method motivated by principles from perceptual organization. To define the suburban, or residential class, we begin by considering the distribution of training data depicted in Fig. 3.3. This space is the most promising one in Bayes classification to be discussed next. Clearly, the residential areas, in which we are most interested, bridge the urban and rural areas in feature space, much as they do on the ground. This makes the direct detection of such areas difficult, but we have been able to make significant inroads on the problem that at least suffice for our "triage".

Our attempt to build a three way Bayes classifier did not give promising results. We suspect that the feature distributions of the urban and rural classes are poorly approximated as single Gaussians. While it is theoretically possible to model these distributions as collections of multiple Gaussians (mixture models), one still needs to know how many Gaussians (subclasses) to fit, and the solution to that problem is far from obvious in the present case. Therefore, we developed an alternative approach to detect suburban regions by framing the problem as one of perceptual grouping.

Perceptual grouping is the process(es) by which vision systems (natural or machine) impose organization on sensory data. The objective is to identify image regions 


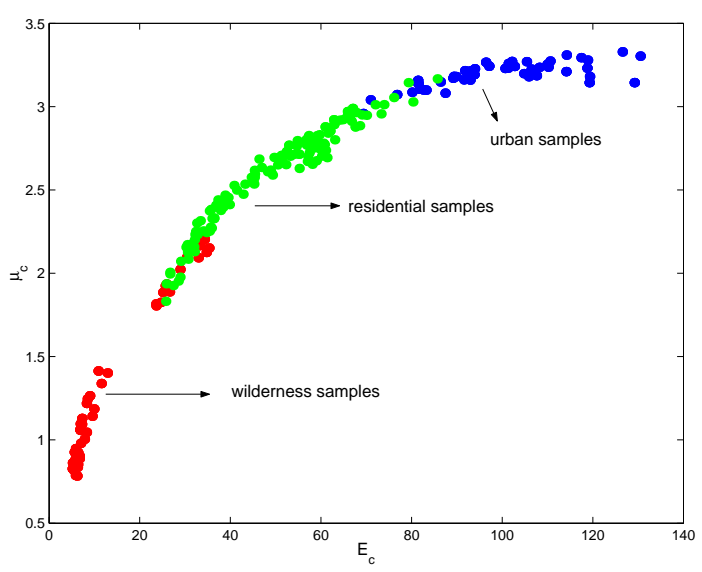

Figure 3.3: Sample distribution in $\mu_{c}, E_{c}$ space

(or features) arising from a common underlying cause. This exploits the fact that the world is not visually chaotic; it has structure and organization. Rather than making hard window by window decisions, we group windows together having compatible (similar) feature values into contiguous regions, and classify them together. Thus, we discover natural groupings of areas having similar developmental characteristics, as measured in the $\left(\mu_{c}, E_{c}\right)$ feature space. The roots of this approach go back to Wuechser and Boyer's [100] robust contour decomposition method. Srikantiah et al. [101] implemented a 2D version of this idea to segment the surfaces of 3D free form objects. We expand this idea to more general feature spaces here.

In grouping, we first construct a $2 \mathrm{D}$ voting space for the feature vectors over the image. Each window votes into all bins satisfying a compatibility constraint. This compatibility constraint takes the form of a $2 \mathrm{D}$ footprint in feature space; any window having feature values in the range covered by this footprint are considered to be compatible with the current (voting) window in that they have similar features. We then interrogate the voting result to extract contiguous regions having compatible 
features, from largest to smallest. The extracted collections of windows are called segments.

In these experiments, the voting spaces are of 16 bins each, uniform over the observed range of the corresponding feature value over the training data. The compatibility constraint is three bins, roughly $20 \%$ of the range. Therefore, of the $16 \times 16=256$ cells in the $2 \mathrm{D}$ voting space, each window votes for 9 cells in a $3 \times 3$ block, that corresponding to its own (quantized) feature vector, and the 8 adjoining cells. At the "corners" of the voting space, the compatibility footprint is simply truncated.

To illustrate the results of the spatial grouping algorithm, we give the Colorado, Sydney, New Mexico, and Ecuador images and the coherent segments obtained from them in Figs. 3.4, 3.5, 3.6, and 3.7.

The feature based grouping method works reasonably well in extracting homogenously developed regions in these images. However, there are some design (and presentation) limitations. Homogeneity is computed only over square windows and is defined in the feature space. Therefore, some groupings may seem inappropriate owing to this spatial quantization. Moreover, the images are printed at different scales (due to their differing sizes). This can be misleading when evaluating the grouping visually.

To assign the final class membership to each segment, we begin by estimating the mean vector $\mu_{\mathbf{x}}$ and covariance matrix $\Sigma_{\mathbf{x}}$ of the suburban class in feature space (green points in Fig. 3.3) assuming a bivariate Gaussian distribution. We compute the Mahalanobis distance between each window to be classified and this distribution: 


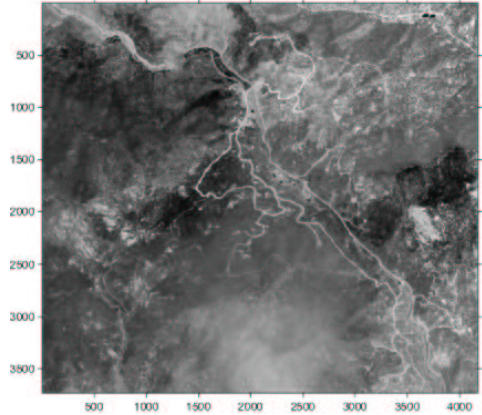

(a) The Colorado image

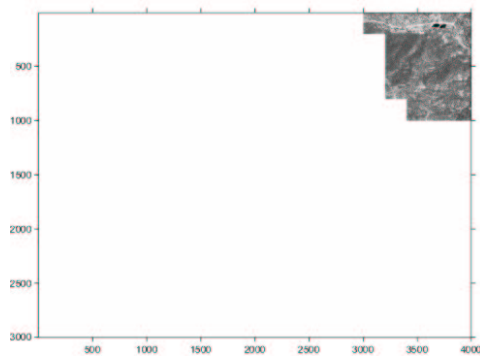

(d) 3. segment

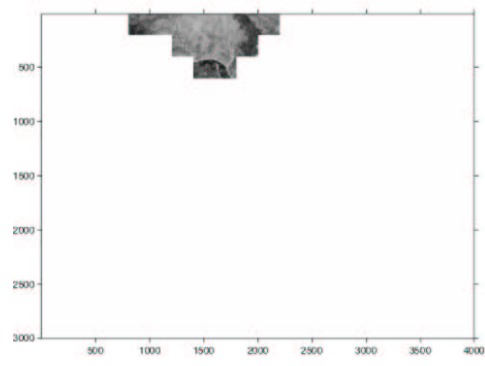

(g) 6. segment

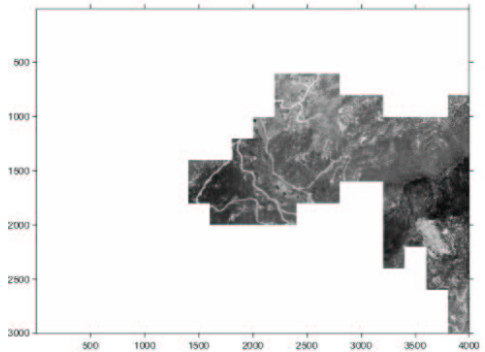

(b) 1. segment

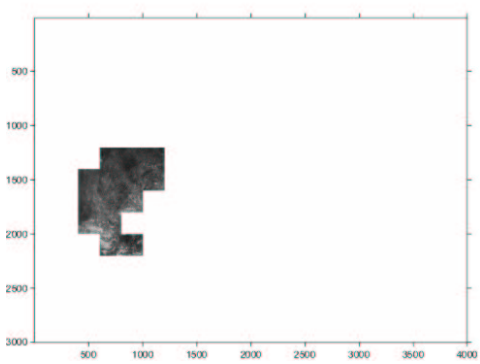

(e) 4. segment

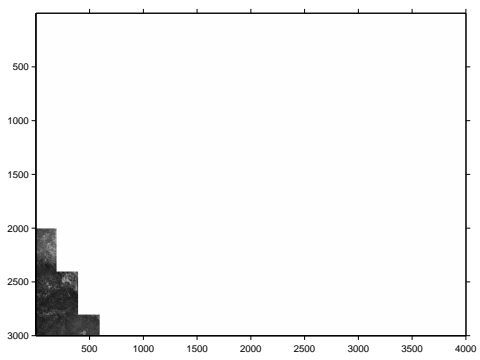

(h) 7. segment

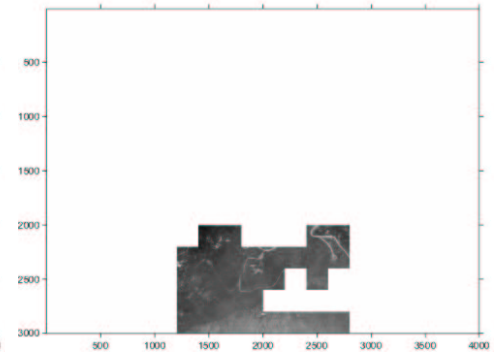

(c) 2. segment

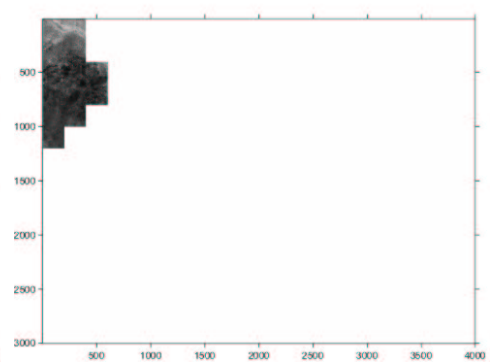

(f) 5. segment

Figure 3.4: The Colorado image and segments obtained from feature based grouping 


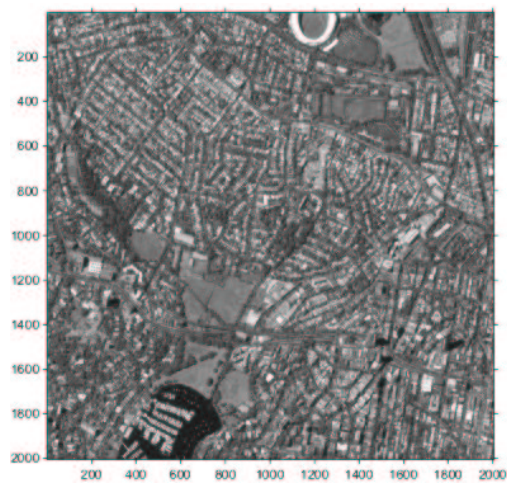

(a) The Sydney image

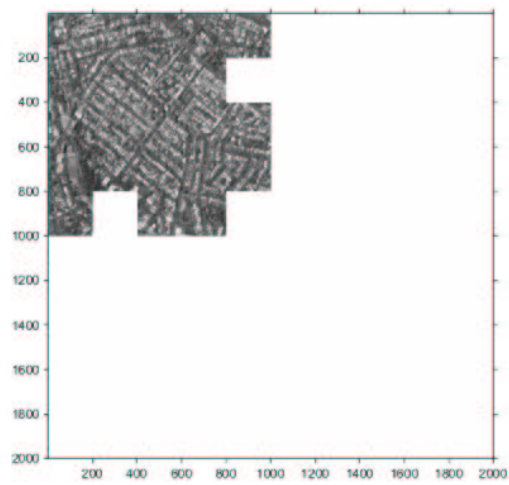

(c) 2. segment

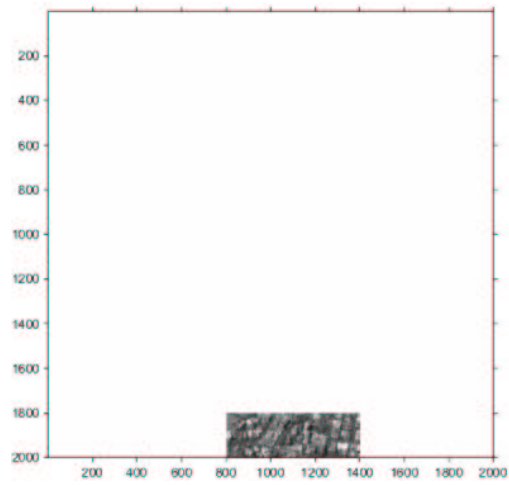

(e) 4. segment

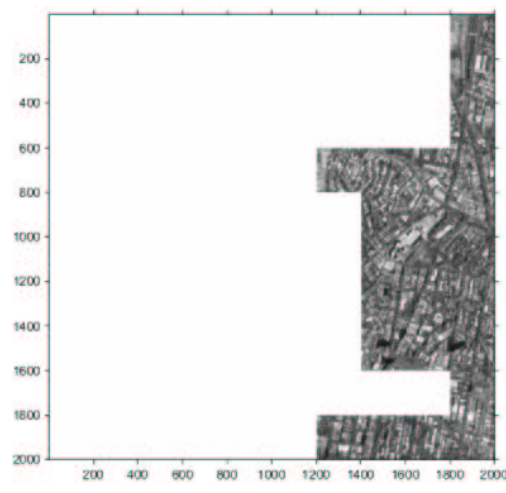

(b) 1. segment

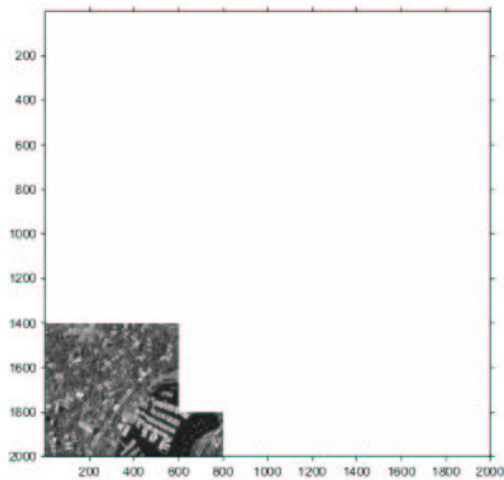

(d) 3. segment

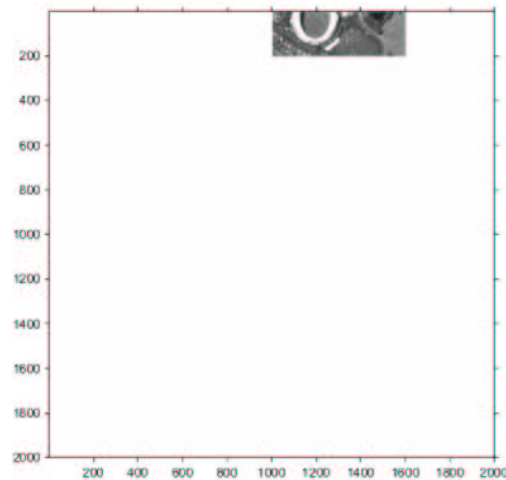

(f) 5. segment

Figure 3.5: The Sydney image and segments obtained from feature based grouping 


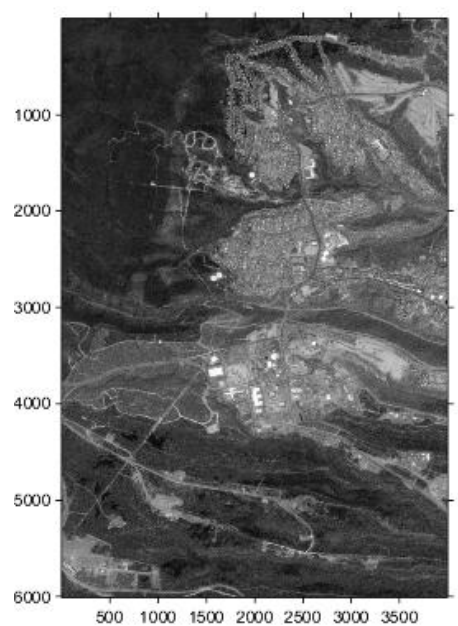

(a) The New Mexico image

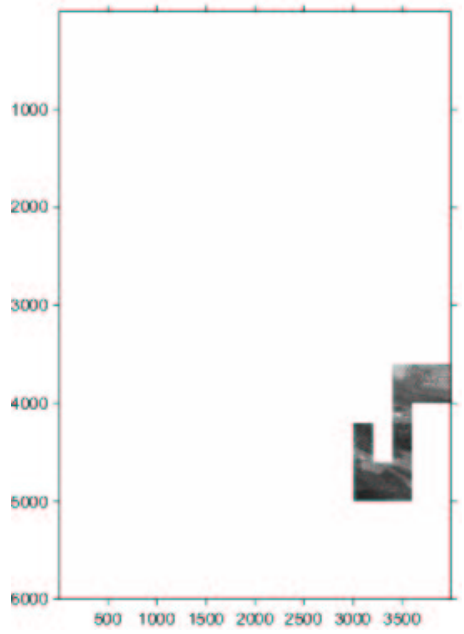

(d) 3. segment

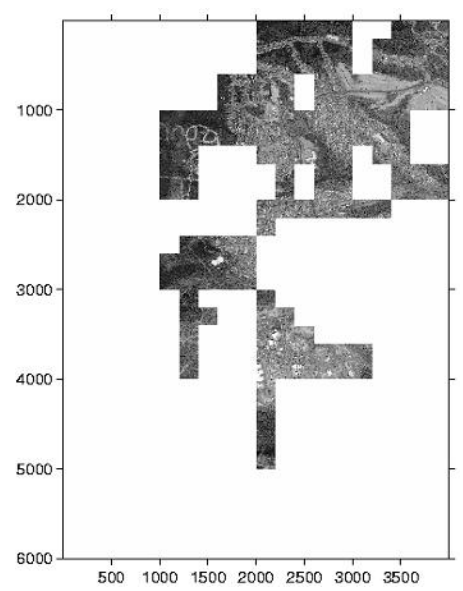

(b) 1. segment

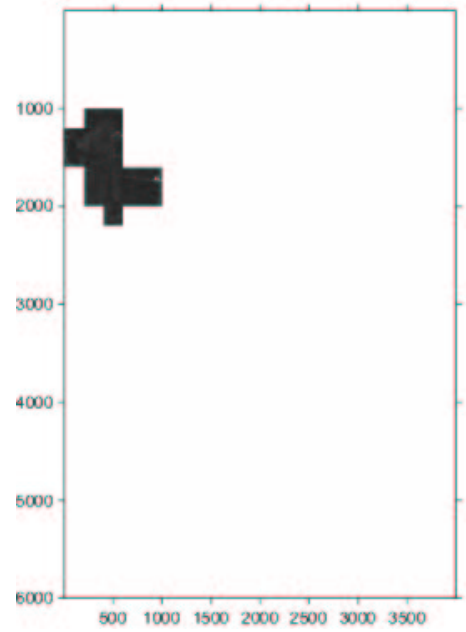

(e) 4. segment

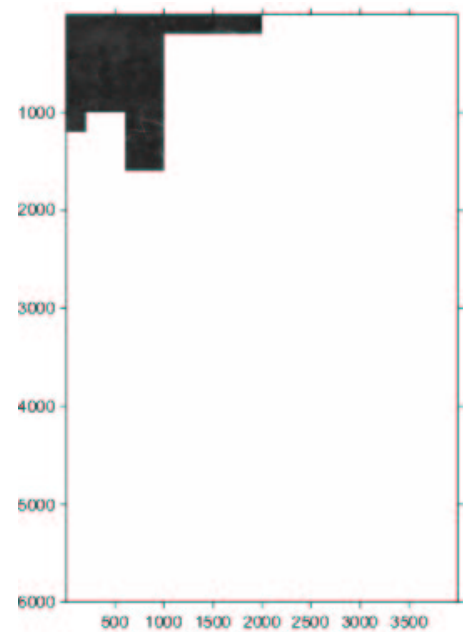

(c) 2. segment

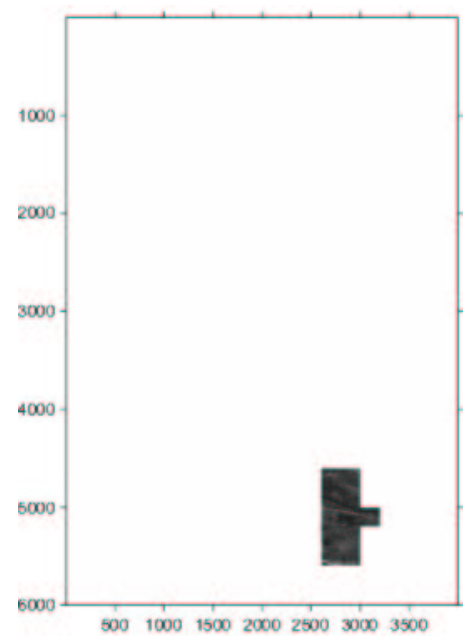

(f) 5. segment

Figure 3.6: The New Mexico image and segments obtained from feature based grouping 


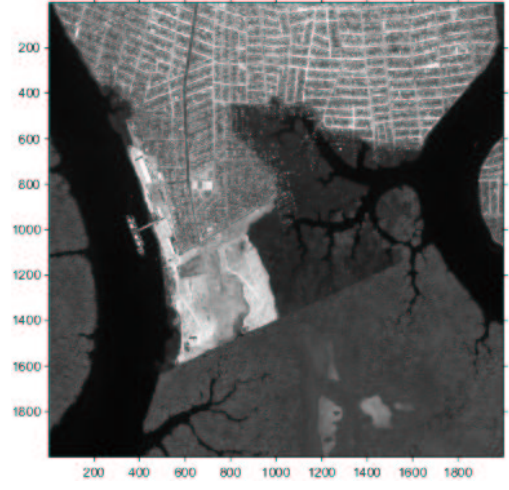

(a) The Ecuador image

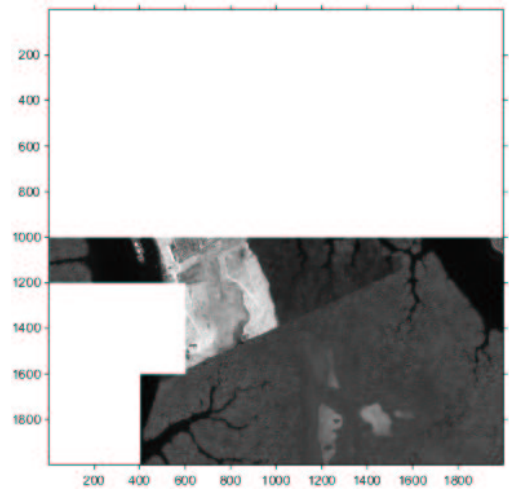

(c) 2. segment

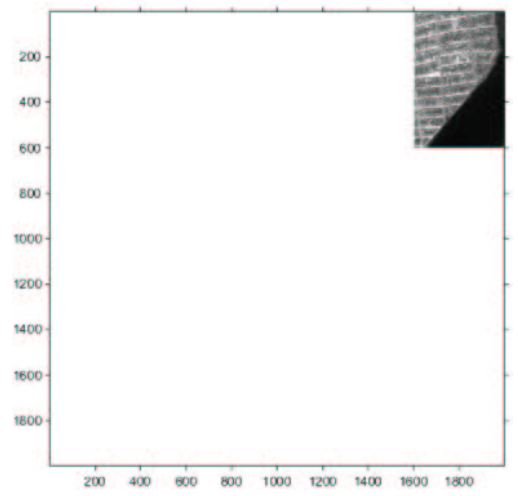

(e) 4. segment

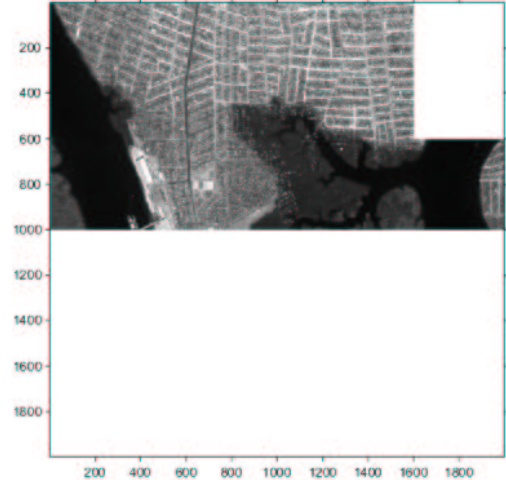

(b) 1. segment

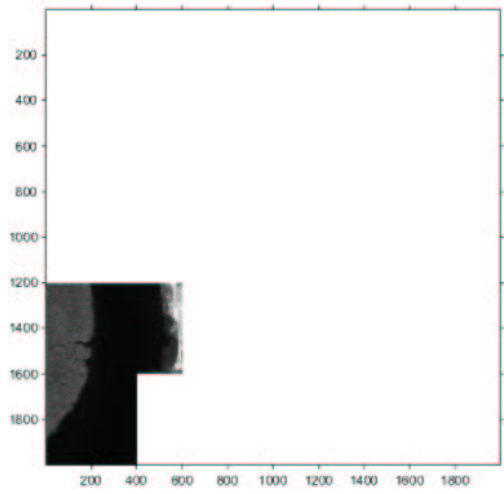

(d) 3. segment

Figure 3.7: The Ecuador image and segments obtained from feature based grouping 


$$
D_{M}=\left(\mathbf{x}-\mu_{\mathbf{x}}\right) \Sigma_{\mathbf{x}}^{-1}\left(\mathbf{x}-\mu_{\mathbf{x}}\right)^{T}
$$

The degree of membership of a given window (or feature vector $\mathbf{x}$ ) is then computed as:

$$
\mathcal{M}=e^{-\frac{D_{M}}{20}}
$$

where the value 20 was selected experimentally for convenience.

We then classify a segment using $\mathcal{M}$. However, a segment's (net) feature vector is that corner of its range corresponding to each feature at its maximum. Although one might expect the average or median value of the feature range to be more representative (as we did), the "upper right" corner value performs better. The reasons are as yet unclear. All windows in the segment then receive the same degree of membership and classification.

\subsection{Experimental Classification Results}

We applied several classification approaches to both the urban-rural two-class problem and the direct detection of suburban areas using the features described, as well as spatial coherence. We implemented Bayes, Parzen window, and nearest neighbor $(\mathrm{NN})$ classifiers [102]. To focus on the most promising classification approach, we only provided the results of Bayes classifier in this section. For complete results for all classifiers in different feature spaces, see the Appendix 3.7. 


\subsubsection{Dataset and Feature Space}

Our dataset consists of 28 one-meter panchromatic images, 18 drawn from across North America, plus 10 from different locations around the world. Table 3.1 summarizes the satellite images used in this study to encompass a wide range of cultures and development patterns. The first 25 images were downloaded from http:// www.spaceimaging.com. Images 26 and 27 were downloaded from http://www-com. iasis.svetcorp.net. Image 28 was downloaded from http://www.visidata.com.

An exhaustive search over the full feature set (all singles, pairs, triples, etc. given in Appendix 3.7) revealed that the best performance is obtained in the $\left(\mu_{c}, E_{c}\right)$ feature space for the Bayes classifier. We used 48 rural and 48 urban image windows selected at random from the overall data set to train each classifier. We tested 2014 new region samples to ascertain the performance of the classifier. A caveat on ground truth: Some windows straddle transitions from developed to undeveloped areas, and some others encompass lightly developed regions, parkland, and other types of terrain that are hard to classify, even manually.

\subsubsection{Two Class Results}

With the given feature space and the Bayes classifier, we obtain a $92.0 \%$ correct classification for the rural regions over 905 test samples. Similarly, we obtain a $83.0 \%$ correct classification for the urban samples over 1109 test samples. The overall classification performance is $\mathbf{8 7 . 0} \%$.

In an attempt to exploit spatial coherence in the two-class case, we applied probabilistic relaxation $[30,103]$ ( for the details, see Chapter 5). We obtain a $91.2 \%$ and $86 \%$ correct classification rates for the rural and urban scenes, with a maximum of 


\begin{tabular}{|l|c|}
\hline Geographic Location & Image Size $(m \times m)$ \\
\hline Colorado, USA & $2002 \times 2002$ \\
\hline Dubai, UAE & $763 \times 953$ \\
\hline California, USA & $640 \times 799$ \\
\hline Colorado, USA & $4177 \times 3733$ \\
\hline New Mexico, USA & $3996 \times 6006$ \\
\hline California, USA & $2001 \times 2001$ \\
\hline Sydney, Australia & $2003 \times 2003$ \\
\hline Colorado, USA & $641 \times 801$ \\
\hline Mississippi, USA & $1992 \times 1995$ \\
\hline Maryland, USA & $7849 \times 3107$ \\
\hline New York, USA & $2000 \times 1997$ \\
\hline Rome, Italy & $1993 \times 1993$ \\
\hline Sanaa, Yemen & $1841 \times 2109$ \\
\hline California, USA & $1992 \times 1996$ \\
\hline Taipei, Taiwan & $2101 \times 2101$ \\
\hline Tokyo, Japan & $1989 \times 2010$ \\
\hline Guayaquil, Ecuador & $1999 \times 1999$ \\
\hline California, USA & $2000 \times 2000$ \\
\hline Egypt & $2104 \times 2104$ \\
\hline London, UK & $2000 \times 2000$ \\
\hline California, USA & $2103 \times 2110$ \\
\hline Sydney, Australia & $1430 \times 1539$ \\
\hline Arizona, USA & $1671 \times 1062$ \\
\hline Colorado, USA & $1121 \times 1112$ \\
\hline Arizona, USA & $2007 \times 2007$ \\
\hline California, USA & $1093 \times 1158$ \\
\hline California, USA & $1156 \times 1160$ \\
\hline North Carolina, USA & $1100 \times 772$ \\
\hline
\end{tabular}

Table 3.1: Test images 
$\mathbf{8 8 . 3} \%$ overall correct classification percentage. Introducing spatial information via probabilistic relaxation improved the correct detection rates slightly; the overall improvement is just $1.3 \%$. More importantly, however, probabilistic relaxation results in more acceptable errors in classification by reducing misses of the urban class.

We tested the feature based grouping approach over the full data set to detect suburban regions. For comparison, we first calculated the class membership for each sample without grouping. We used a Neyman-Pearson decision rule (specify the false alarm rate, then obtain the corresponding best detection rate) [104], yielding the receiver operating characteristic (ROC) labelled "Before grouping" in Fig. 3.8. This ROC was obtained by testing all of our samples. Although cast as a two class problem (suburbs, or not), this is really a three class issue with fairly unequal priors, and so the ROC - while not spectacular - is far better than chance. It also makes no use (yet) of spatial coherence.

\subsubsection{Suburban Area Detection Results}

We tested the feature based grouping approach over the full data set to detect suburban regions using the membership parameter $\mathcal{M}$. For comparison, we first calculated the class membership for each sample without grouping. We used a NeymanPearson decision rule (specify the false alarm rate, then obtain the corresponding best detection rate) [104], yielding the receiver operating characteristic (ROC) labelled "Before grouping" in Fig. 3.8. This ROC was obtained by testing all of our samples. Although formulated as a two class problem (suburbs, or not), this is really a three class issue with fairly unequal priors (suburbs are relatively rare in this 
dataset), and so the ROC - while not spectacular - is far better than chance. It also makes no use (yet) of spatial coherence.

Next, we applied feature based grouping. Again, using a Neyman-Pearson decision rule we obtained the "After grouping" ROC curve in Fig. 3.8 which reveals substantially better detection rates for all false alarm rates specified. This comparison shows the improvement available from feature based grouping.

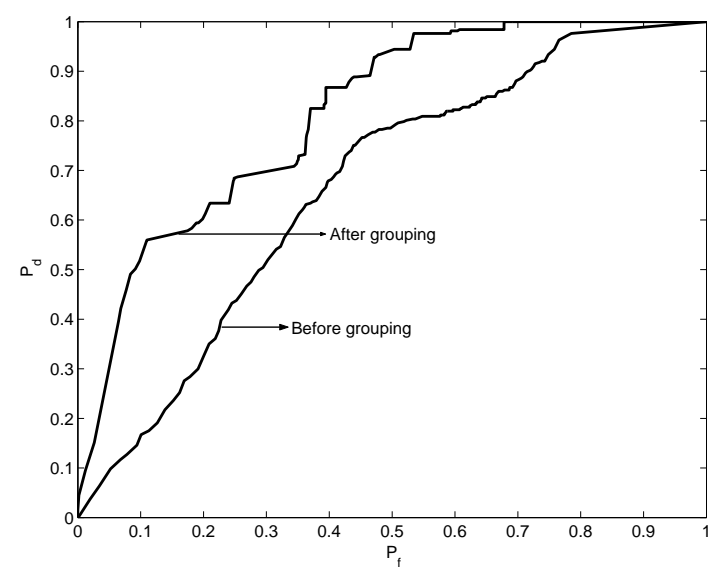

Figure 3.8: ROC curves for suburbia

The area under a ROC curve gives a single number for quality and improvement, and for the perfect case it would be one. If we compare areas under these two ROC curves, we obtain 0.6688 from the before grouping ROC curve and 0.8165 from the after grouping ROC curve. This also indicates an improvement of approximately $22 \%$ by applying feature based grouping. However, it remains difficult to achieve high detection rates without significant false alarms. 


\subsection{Summary of the Overall Classification System}

In the previous sections, we discussed each step separately, together with their parameter values. Here, we tabulate the parameters with respect to their nominal values and relative influence on the final performance (sensitivity) in Table 3.2.

\begin{tabular}{|l|c|c|c|c|}
\hline Step & Section & Parameter & Value & Influence \\
\hline Line extraction & gradient calculation & $\alpha$ & 1 & high \\
Line extraction & gradient calculation & $\beta$ & 70 & low \\
Line extraction & pixel grouping & binsize & $\pi / 4$ & medium \\
Feature extraction & length & binsize & 37 & low \\
Feature extraction & contrast & binsize & 95 & low \\
Feature based grouping & voting space & binsize & 16 & medium \\
Feature based grouping & degree of membership & coefficient & 20 & low \\
Classification & Bayes classifier & training set & 96 & medium \\
\hline
\end{tabular}

Table 3.2: Summary of the system parameters

Although there are eight parameters in the system, only the value of the gradient scale parameter $\alpha$ has a major impact. If the lines can not be extracted effectively, all remaining calculations are compromised. The binsize in pixel grouping (in line extraction step) has a medium effect through its influence on the lines extracted. Binsizes in the feature extraction step have only a weak impact on the final classification result because statistical measures summarize these histograms as a whole; no individual entry is critical.

\subsection{Appendix: Additional Features}

We considered three features beyond those of Section 3.2. These are less powerful than the length and contrast based features and require more computation. Their 
primary limitation is that they depend heavily on city models that do not necessarily hold. However, they do provide reasonable classification performance and may be more useful in specific domains.

- Orientation distribution, $R_{o}$

- Entropy of line spacing, $E_{s}$

- Periodicity of line placement, $P$

\subsubsection{Orientation}

The orientation feature $R_{o}$ is the crosscorrelation between the histogram of straight line orientations in a given image window with an idealized bimodal density function model for local urban areas, having two Gaussian modes separated by $\pi / 2$ radians. Since the feature is calculated over a window, this assumption need not to hold for the overall image as is in the previous features. This idealized model works well as long as there is no single dominant mode in street direction in the image window. We build a weighted (based on line length) orientation histogram. We use weighted voting because lines obtained from small line support regions do not have reliable orientations. The orientation range is quantized to 32 bins (with binwidth $=0.03 \pi$ radians), where the first bin is centered at $-\pi / 2$ radians and the last bin at $\pi / 2$ radians. As in the length and contrast features, the bin size is not crucial.

Let the normalized vote for bin $i$ be $h_{o}(i)$ for $i=1, \ldots, 32$. We cross correlate the normalized orientation histogram with the bimodal density function model $g(t)$ :

$$
g(t)=\frac{1}{2 \sqrt{0.2 \pi}}\left[\exp \left(\frac{-1}{2}\left(\frac{t+\pi / 4}{0.1}\right)^{2}\right)+\exp \left(\frac{-1}{2}\left(\frac{t-\pi / 4}{0.1}\right)^{2}\right)\right]
$$


The denominator of 0.1 in the bimodal density function (actually the standard deviation for each unimodal part) provides a tolerance for the parallelness of straight lines in the image window. This value is obtained from the layout of the buildings in images.

The crosscorrelation value given in Eqn. 3.19 indicates the similarity of the image window to the idealized urban region model while the shift $t$ at which the maximum correlation occurs indicates the dominant street grid orientation. This could prove useful in subsequent analyses.

$$
R_{o}=\max _{t}\left\{g(t) \otimes h_{o}(t)\right\}
$$

\subsubsection{Line Spacing}

This feature captures the spatial distribution of lines in the image window. We represent each line by its center of mass and obtain an entropy measure from the distribution of line spacings. For each line, we locate the centers of mass of the nearest two lines of similar length ( \pm 3 pixels). The spacing between the original line and each neighbor is taken to be the Euclidean distance between their centers of mass. We repeat this calculation for all lines in the given window.

We compute the histogram of line spacings over 70 bins (with binwidth $=2$ pixels), with the first bin centered at 2 pixels and the last at 140 pixels. Let the normalized vote for bin $i$ be $h_{\text {dcom }}(i)$ for $i=1, \ldots, 70$. The entropy of line spacing, $E_{s}$, is then obtained from Eqn. 3.14. 


\subsubsection{Periodicity}

Most urban regions (since the early 20th century) are constructed in accordance with a (set of) zoning plan(s). Therefore, we expect more grid like structures (such as blocks of buildings) and more periodic substructures in urban regions. This assumption is often violated for older cities, especially outside North America. Therefore, this feature is not a strong one, in general. However, if one's attention is confined to North America (or Australia) where land development continues apace, this feature may prove useful.

Straight lines extracted from well-organized structures should exhibit some form of periodicity in their placement. We infer periodicity through the projection of the line segments onto each of four axes: $y=0, y=x, x=0$, and $y=-x$; the origin is at the center of the window. To make the discussion concrete, let's consider the case for the $x=0$ (that is, the $y$ ) axis. The intersection point of each straight line segment (or its extension) with the line $x=0$ is identified. A function is defined along this axis whose value at each line segment intersection point is the number of pixels in the line segment(s) projecting to that point. Non-intersection points (points not projected to by any line segment in the window) are assigned the value 0. Fig. 3.9 illustrates the

procedure for the case of three line segments, one of length $L_{1}$ projecting to location $y=y_{1}$ on the $x=0$ axis, and two each of length $L_{2}$ projecting to $y=y_{2}$ and $y=y_{3}$, respectively.

The resulting set of four projection functions are then analyzed for periodicity. Because projections along directions highly oblique to the axis are unreliable, we consider four axes (as mentioned above) and limit the set of projecting line segments 
to be those oriented within $\pm 45^{\circ}$ of the direction orthogonal to the current projection axis.

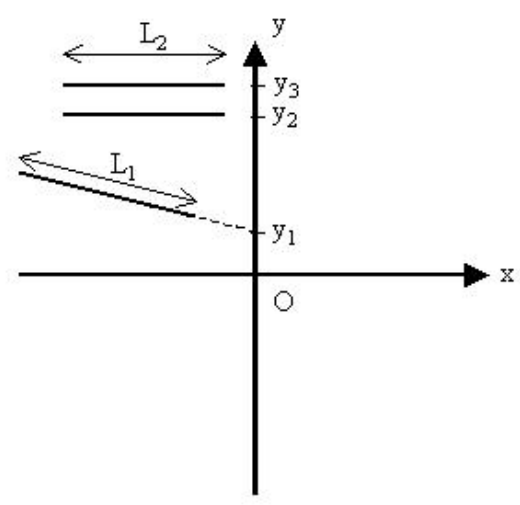

(a) Parallel lines and $x-y$ axes

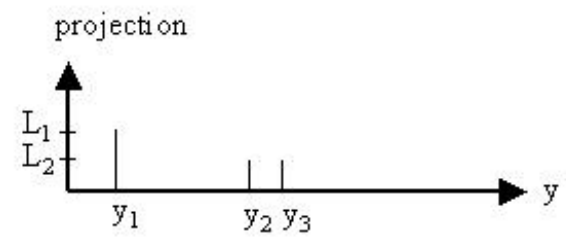

(b) Projection of parallel lines on y axis

Figure 3.9: An example of the projection of lines

We use the periodicity transform introduced by Sethares and Staley [105] to measure the periodicity of the projection function. They project the given one dimensional signal onto non-orthogonal periodic subspaces using an M-best periodic decomposition algorithm. We find that the transform coefficient for the first periodic subspace sufficiently represents the periodicity in the projection function. The maximum periodicity $P$ among the four projection functions is taken as the periodicity of the image window. 


\subsection{Appendix: More on Classification}

Now, we summarize two additional sets of findings with respect to classification. First, we consider the Bayes, Parzen window, and nearest neighbor (NN) classifiers. Then, we explore their performances on the overall feature space. Therefore we will give the reason why we used the Bayes classifier and the feature space $\left(\mu_{c}-E_{c}\right)$ in Section 3.4.

We start by tabulating the best classification performances of each classifier in different spaces in Tables 3.3, 3.4, and 3.5. In these tables, each row of each table gives the best set of $n$ features (the best n-D feature space) for $n \in[1,7]$.

\begin{tabular}{|l|c|}
\hline Feature Combination & Performance \\
\hline$E_{c}$ & 86.0 \\
\hline$E_{c}, \mu_{c}$, & $\mathbf{8 7 . 0}$ \\
\hline$E_{c}, \mu_{c}, R_{o}$ & 86.6 \\
\hline$E_{c}, \mu_{c}, R_{o}, E_{s}$ & 86.5 \\
\hline$E_{c}, \mu_{c}, R_{o}, E_{s}, E_{l}$, & 86.6 \\
\hline$E_{c}, \mu_{c}, R_{o}, E_{s}, E_{l}, P$, & 86.6 \\
\hline$E_{c}, \mu_{c}, R_{o}, E_{s}, E_{l}, P, \mu_{l}$ & 86.4 \\
\hline
\end{tabular}

Table 3.3: Performances for best feature spaces for the Bayes classifier

We see that there is a different best feature space for each classifier, but the performances of the classifiers are not statistically different from one another. However, the computational costs of the classifiers, and of the best features for each, do vary. We can use computational cost and performance together to select the best feature space-classifier combination. 


\begin{tabular}{|l|c|}
\hline Feature Combination & Performance \\
\hline$E_{c}$ & 87.1 \\
\hline$E_{c}, E_{l}$ & $\mathbf{8 7 . 3}$ \\
\hline$\mu_{c}, E_{s}, P$ & 83.5 \\
\hline$E_{c}, \mu_{c}, E_{l}, \mu_{l}$ & 75.4 \\
\hline$E_{c}, \mu_{c}, E_{l}, \mu_{l}, E_{s}$ & 81.5 \\
\hline$E_{c}, \mu_{c}, E_{l}, E_{s}, P, R_{o}$ & 82.8 \\
\hline$E_{c}, \mu_{c}, E_{l}, \mu_{l}, E_{s}, P, R_{o}$ & 82.3 \\
\hline
\end{tabular}

Table 3.4: Performances for best feature spaces for the Parzen window classifier

\begin{tabular}{|l|c|}
\hline Feature Combination & Performance \\
\hline$E_{c}$ & 87.6 \\
\hline$E_{c}, E_{l}$ & $\mathbf{8 7 . 8}$ \\
\hline$E_{c}, E_{s}, R_{o}$ & 84.5 \\
\hline$E_{l}, E_{s}, R_{o}, \mu_{c}$ & 84.0 \\
\hline$E_{c}, E_{l}, R_{o}, \mu_{c}, P$ & 83.2 \\
\hline$E_{c}, E_{l}, R_{o}, \mu_{c}, P, E_{s}$ & 83.2 \\
\hline$E_{c}, E_{l}, R_{o}, \mu_{c}, P, E_{s}, \mu_{l}$ & 82.8 \\
\hline
\end{tabular}

Table 3.5: Performances for best feature spaces for the NN classifier

Next, we tabulate the classification performances for all 1D and 2D feature spaces for each classifier in Tables 3.6, 3.7, and 3.8. In these tables, diagonal cells represent the classification performance of each feature alone, off-diagonal cells represent the classification performance of the corresponding 2D feature space.

These tables show that the length and contrast based features are highly effective for classification. However, although they perform above the chance line, the rotation and projection features perform poorly relative to the others.

Finally, we provide the classification performance (rural, urban, and overall) as well as the best feature space for each classifier separately in Table 3.9. 


\begin{tabular}{|c|c|c|c|c|c|c|c|}
\hline & $\mu_{c}$ & $E_{c}$ & $\mu_{l}$ & $E_{l}$ & $E_{s}$ & $P$ & $R_{o}$ \\
\hline$\mu_{c}$ & 85.8 & $\mathbf{8 7 . 0}$ & 83.9 & 85.9 & 79.7 & 80.5 & 82.4 \\
\hline$E_{c}$ & & 86.0 & 84.0 & 86.0 & 79.1 & 77.9 & 84.5 \\
\hline$\mu_{l}$ & & & 82.7 & 83.9 & 83.5 & 83.3 & 84.1 \\
\hline$E_{l}$ & & & & 86.0 & 84.5 & 86.4 & 84.9 \\
\hline$E_{s}$ & & & & & 72.4 & 77.1 & 73.3 \\
\hline$P$ & & & & & & 68.2 & 68.6 \\
\hline$R_{o}$ & & & & & & & 67.8 \\
\hline
\end{tabular}

Table 3.6: The Bayes classification results in percentages for all $1 \mathrm{D}$ and $2 \mathrm{D}$ feature spaces

\begin{tabular}{|c|c|c|c|c|c|c|c|}
\hline & $\mu_{c}$ & $E_{c}$ & $\mu_{l}$ & $E_{l}$ & $E_{s}$ & $P$ & $R_{o}$ \\
\hline$\mu_{c}$ & 84.7 & 86.8 & 83.4 & 85.5 & 81.2 & 81.5 & 78.3 \\
\hline$E_{c}$ & & 87.1 & 85.4 & $\mathbf{8 7 . 3}$ & 83.2 & 82.3 & 83.9 \\
\hline$\mu_{l}$ & & & 78.3 & 81.6 & 79.7 & 79.2 & 72.8 \\
\hline$E_{l}$ & & & & 82.7 & 83.5 & 84.2 & 76.5 \\
\hline$E_{s}$ & & & & & 72.9 & 73.3 & 72.6 \\
\hline$P$ & & & & & & 65.9 & 62.5 \\
\hline$R_{o}$ & & & & & & & 58.8 \\
\hline
\end{tabular}

Table 3.7: The Parzen window classification results in percentages for all 1D and 2D feature spaces

\begin{tabular}{|c|c|c|c|c|c|c|c|}
\hline & $\mu_{c}$ & $E_{c}$ & $\mu_{l}$ & $E_{l}$ & $E_{s}$ & $P$ & $R_{o}$ \\
\hline$\mu_{c}$ & 85.8 & 85.9 & 85.9 & 85.9 & 85.9 & 85.9 & 85.9 \\
\hline$E_{c}$ & & 87.6 & 84.4 & $\mathbf{8 7 . 8}$ & 87.1 & 76.2 & 88.0 \\
\hline$\mu_{l}$ & & & 81.6 & 81.7 & 82.6 & 79.6 & 81.4 \\
\hline$E_{l}$ & & & & 85.0 & 83.7 & 71.4 & 83.6 \\
\hline$E_{s}$ & & & & & 72.4 & 68.7 & 74.1 \\
\hline$P$ & & & & & & 61.2 & 63.3 \\
\hline$R_{o}$ & & & & & & & 63.7 \\
\hline
\end{tabular}

Table 3.8: The NN classification results in percentages for all $1 \mathrm{D}$ and $2 \mathrm{D}$ feature spaces 


\begin{tabular}{|l|c|c|c|c|}
\hline & & \multicolumn{3}{|c|}{ Performance (\%) } \\
\cline { 3 - 5 } Classifier & Feature space & rural & urban & average \\
\hline Bayes & $\mu_{c}, E_{c}$ & 92.0 & 83.0 & 87.0 \\
\hline Parzen window & $E_{c}, E_{l}$ & 89.3 & 85.7 & 87.3 \\
\hline NN & $E_{c}, E_{l}$ & 91.0 & 85.2 & 87.8 \\
\hline
\end{tabular}

Table 3.9: Comparison of performances for the three classifiers

Each classifier presents both benefits and shortcomings. The Bayes classifier comes at low computational cost, but assumes a parametric model for the data distribution, nearly always Gaussian. If the data represent such a characteristic, the classifier performs well. If not, it may be possible to model the data as a mixture of multiple Gaussians; we have not pursued this additional complexity in the present work. The Parzen window and NN classifiers do not assume any particular a priori distribution; they model the data distribution locally. The main drawback of these two methods is their computational cost. Although they may represent non Gaussian distributions more robustly than the Bayes classifier, they did not perform appreciably better on our data. Therefore, we recommend the Bayes classifier in this domain.

\subsection{Summary of the Chapter}

We presented a system to discriminate developed and wilderness regions in this chapter. We based the assessment on the photometric and geometric characteristics of straight line segments. From the straight line segments we extracted statistical features based on orientation, length, periodicity, contrast, and line spacings. By using parametric and non-parametric classifiers we were able to distinguish developed areas with high confidence. Next, we will consider multispectral information in 
classification. Before that step, we improve the multispectral measures in the next chapter. 


\section{CHAPTER 4}

\section{The Multispectral Information: Vegetation and Shadow-Water Indices}

In this chapter, we focus on vegetation indices to extract the multispectral information in subsequent processing (especially land use classification and house street network extraction). Vegetation indices have been used extensively to estimate the vegetation density from satellite and airborne images for many years.

Nearly all plants need sunlight to survive, having chlorophyll to convert radiant energy from the sun to organic energy. Chlorophyll exhibits unique absorption characteristics, absorbing wavelengths around the visible red band, $645 \mu \mathrm{m}$, while being transparent to wavelengths in the near-infrared, $700 \mu \mathrm{m}$. These characteristics of chlorophyll are commonly used to design indices to estimate local vegetation density in multispectral satellite or airborne imagery.

We first consider two popular vegetation indices based on the above observations: the normalized difference vegetation index $(N D V I)$ and the ratio vegetation index $(R V I)$. Although there have been many indices proposed in the literature, the NDVI remains one of the most popular in remote sensing applications. Other well-known vegetation indices can be found in [106-114]. Most of these represent attempts to overcome deficiencies in the NDVI. 
Previous studies have compared vegetation indices based on various criteria [115123], yet no clear winner has emerged. In some experiments, the NDVI outperforms all other indices; for others it is less successful. It is clear that, despite many attempts to develop alternatives, the NDVI remains one of the most effective indicators of vegetation density.

Since its introduction, the NDVI has been used in a wide variety of studies including those on global vegetation [124-127], crop estimation and vegetation growth [128-132], land cover [133-142], early famine detection [143], and climate [144,145].

Nevertheless, two main objections have been raised with respect to the NDVI. First, it has been argued that the NDVI is ad-hoc $[146,147]$, and our extensive literature review failed to turn up any claim to the contrary. Second, the NDVI is highly nonlinear, saturating for highly vegetated areas. Many authors have mentioned this saturation problem [111,148-152]. Although used extensively in practice, these two problems limit scientific confidence in the NDVI. We address these two problems in this paper.

We first introduce a statistical framework for the NDVI, to stress that it has a rigorous explanation. That is, even if its original definition was heuristic or ad-hoc in nature, it is nevertheless possible to provide a sound theoretical justification for the index. To our knowledge, there have been no studies establishing a statistical foundation for the NDVI.

We then propose a solution to the nonlinearity (saturation) problem based on our statistical explanation. By representing the NDVI as a slope and obtaining its inverse tangent, we are able to linearize the measure to yield a new index. We represent simulation and real image results to validate our claim. 
Our statistical framework also points to a relationship between the $R V I$ and the $N D V I$. We show that the NDVI and the RVI capture exactly the same information. That is, they calculate the same slope, but in two different representations of the same space, one representation being a rotated version of the other.

For completeness, we applied our statistical framework to the 3D and 4D feature spaces of blue, green, red, and near-infrared bands. The original use of a 2D feature space was based on physical arguments alone. We develop vegetation indices based on slope definitions in the 3D and 4D transformed spaces similar to the NDVI. Not surprisingly, these new indices were not as powerful as the NDVI. However; in these new indices, we can use information in the blue band to eliminate the atmospheric effects observed by many authors.

Finally, we introduce a group of shadow-water indices to measure shadow and water density in multispectral images with the same framework. Shadows affect the quality of vegetation density estimates in remote sensing. Shadows are also extremely important indicators of building height from a single image. Shadow and water sources affect the blue band more than do other objects in satellite images. Using this information in our statistical framework, we are able to estimate shadow and water density from multispectral images. We apply our shadow-water indices on real images to validate our claims.

\subsection{Background and Historical Development}

The pioneering work of Shull [153] examined the reflectance and absorption characteristics of leaves. Rabideau et al. [154], Billings and Morris [155], and Federer and Tanner [156] conducted experiments that indicated the same reflectance-absorption 
characteristics. Weidner et al. [157] conducted experiments on various types of plants, (plants having thick leaves, thin leaves, dark green leaves, and desert plants) to show that these absorption characteristics are highly consistent across plant types.

Based on these observations, Jordan [158] introduced the ratio vegetation index $(R V I)$ to estimate the vegetation density in a given region. Since absorption is high in the red band and low in the near-infrared, Jordan assessed vegetation density using the ratio:

$$
R V I=\frac{\rho_{\text {nir }}}{\rho_{\text {red }}}
$$

where $\rho_{\text {nir }}$ and $\rho_{\text {red }}$ are reflectance values in near-infrared and red bands respectively. Colwell $[159,160]$ applied the $R V I$ to grass canopies and found it useful for estimating biomass. One of the drawbacks of the $R V I$ is that it diverges to infinity when $\rho_{\text {red }}$ goes to zero.

Nalepka et al. [161] introduced the square root of the $R V I$ (SRRVI), as a new vegetation index:

$$
S R R V I=\sqrt{R V I}=\sqrt{\frac{\rho_{\text {nir }}}{\rho_{\text {red }}}}
$$

This index is more linear than the RVI. Similarly Rouse et al. [162] introduced the transformed vegetation index $(T V I)$ :

$$
T V I=\sqrt{R V I+0.5}
$$

At this point, we have to mention that the $R V I$ saturates as the vegetation density exceeds a limit. Tucker [163] in his paper gave examples of this saturation. Both 
SRRVI and TVI were heuristics designed to mitigate the saturation problem. In this chapter, we propose a more rigorous solution to this problem.

In 1974, Rouse et al. [162] introduced the normalized difference vegetation index $(N D V I)$. This index, given in Eqn. 4.4, remains one of the most popular vegetation indices of all:

$$
N D V I=\frac{\rho_{\text {nir }}-\rho_{\text {red }}}{\rho_{\text {nir }}+\rho_{\text {red }}}
$$

Another vegetation index calculation method is introduced by Kauth and Thomas [106]. They introduced a transformation using four Landsat MSS bands $\left(d_{4}, d_{5}, d_{6}\right.$, $\left.d_{7}\right)$. Band $5\left(d_{5}\right)$ is red; bands 6 and $7\left(d_{6}, d_{7}\right)$ are near-infrared. The Kauth-Thomas transformation is as follows:

$$
\left[\begin{array}{l}
b \\
g \\
y \\
n
\end{array}\right]=\left[\begin{array}{cccc}
0.433 & 0.632 & 0.586 & 0.264 \\
-0.290 & -0.562 & 0.600 & 0.491 \\
-0.829 & -0.522 & -0.039 & 0.194 \\
0.223 & 0.012 & -0.543 & 0.810
\end{array}\right] \times\left[\begin{array}{c}
d_{4} \\
d_{5} \\
d_{6} \\
d_{7}
\end{array}\right]+\left[\begin{array}{l}
32 \\
32 \\
32 \\
32
\end{array}\right]
$$

In the transformed space, $b$ stands for 'brightness', $g$ stands for 'greenness', $y$ stands for 'yellowness', and $n$ stands for 'nonesuch'. Greenness is taken as a vegetation index from this transformation. In his comprehensive analysis of Landsat range and biomass monitoring, Deering [164] mentioned that the NDVI and the TVI are more significant than the vegetation index obtained from the Kauth-Thomas transformation. Jackson [165] extended the ideas of Kauth and Thomas by using n-bands. His method depends on the Gram-Schmidt orthogonalization process [166]. These transformation methods, especially the Gram-Schmidt orthogonalization, are the closest to ours. The difference between these methods and ours is the way we obtain orthogonal spaces and the use of those spaces. 
In the following sections, we first explain the NDVI from a statistical point of view. Then with the tools we develop, we introduce new vegetation and shadow-water indices. To lay the theoretical groundwork, we first review key statistical methods.

\subsection{Statistical Preliminaries}

In this section, we briefly explain three key ideas from statistics. The first is principal components analysis (PCA). We will use PCA to obtain uncorrelated random variables. The second idea is parameter estimation via mode matrix. We will estimate the PCA parameters from a large sample set.

\subsubsection{Principal Components Analysis (PCA)}

PCA performs a linear transformation on a set of correlated random vectors to represent them in a new space such that they are uncorrelated. The new coordinate space is related to the original by rotation about the origin, such that the new coordinate axes are aligned with the dominant directions of dispersion (the principal components) of the data, as inferred from its covariance matrix. Our description here is necessarily tense; for more detail, see [73].

Let the original (correlated) random vectors be represented as $\mathbf{x}_{1}, \mathbf{x}_{2}, \ldots, \mathbf{x}_{n}$. The linear transformation matrix $\mathbf{A}$ is calculated as follows. First, we form the data matrix $\mathbf{X}$ :

$$
\mathbf{X}=\left(\mathbf{x}_{1}, \mathbf{x}_{2}, \ldots, \mathbf{x}_{n}\right)
$$


$\mathbf{X}$ is therefore a $d \times n$ matrix, where $d$ is the dimensionality of the measurement space, and $n$ is the number of data vectors (observations). From this, we obtain the sample covariance matrix $\mathbf{C}_{x}$ :

$$
\mathbf{C}_{x}=E\left[\left(\mathbf{X}-\mu_{\mathbf{x}}\right)\left(\mathbf{X}-\mu_{\mathbf{x}}\right)^{T}\right]
$$

where $\mu_{\mathbf{x}}$ is the sample mean vector, given by $\frac{1}{n} \sum_{i} \mathbf{x}_{i}$.

We then find the eigenvectors $\mathbf{e}_{i}$ of the sample covariance matrix, which satisfy:

$$
\mathbf{C}_{x} \mathbf{e}_{i}=\lambda_{i} \mathbf{e}_{i} \quad i=1,2, \ldots, d
$$

In accordance with standard practice, we index eigenvalue-eigenvector pairs such that $\lambda_{1} \geq \lambda_{2} \geq \ldots \geq \lambda_{d}$. We also normalize the eigenvectors such that $\left\|\mathbf{e}_{i}\right\|=1 \forall i$. The value of $\lambda_{i}$ corresponds to the mean-square excursion of the data (with respect to its mean) along the direction of $\mathbf{e}_{i}$. The $d \times d$ transformation matrix $\mathbf{A}$ is then formed by stacking the eigenvectors:

$$
\mathbf{A}=\left[\begin{array}{c}
\mathbf{e}_{1}^{T} \\
\mathbf{e}_{2}^{T} \\
\vdots \\
\mathbf{e}_{d}^{T}
\end{array}\right]
$$

Applying this transformation to any given data vector $x_{j}$ is simply a matter of projecting $x_{j}$ onto each eigenvector:

$$
\mathbf{p}_{j}=\mathbf{A}\left(\mathbf{x}_{j}-\mu_{\mathbf{x}}\right)
$$

For our derivations, we shift the principal components by transformed means as follows: 


$$
\mathbf{p c}_{j}=\mathbf{A} \mathbf{x}_{j}
$$

where $\mathbf{p} \mathbf{c}_{j}=\mathbf{p}_{j}+\mathbf{A} \mu_{\mathbf{x}}$; we therefore work in non-centered spaces.

\subsubsection{Parameter Estimation via Mode Matrix}

Ideally one could obtain the transformation matrix $\left(\mathbf{A}=\left[a_{i j}\right]\right)$ by using all the observations. However, the size of our training set (5905 images $\times 200$ pixels $\times$ 200 pixels) does not allow such direct calculation. Therefore, we estimate the transformation matrix $\left(\mathbf{A}=\left[a_{i j}\right]\right)$ statistically. Unfortunately, estimating each matrix entry $a_{i j}$ separately is not suitable, since there is an orthogonality constraint on $\mathbf{A}$ (entry $a_{i j}$ is not independent of the others).

To solve this problem, we introduce the notion of a mode matrix. For this notion we take each matrix as a single entity. We then obtain the sample distribution of these entities. We then pick the entity (matrix) with highest value in the distribution as the mode matrix. Since we keep each matrix unchanged during calculations, our mode matrix will be orthogonal.

\subsubsection{Entropy of a Distribution}

To compare different vegetation indices, we will use the notion of entropy given in Eqn. 3.14. The entropy increases as the distribution becomes more uniform.

To use this property in comparison, we pick test images (such as residential region images) having high contrast between their vegetated and non-vegetated regions. Then, we obtain the approximate probability mass function (pmf) of each vegetation 
index for such an image. We expect a fairly uniform pmf since we picked a high contrast image. Finally, we calculate the entropy of each pmf corresponding to different vegetation indices. The higher its entropy, the better we believe that index will be.

Let an approximate pmf be given as $h(i)$ for $i=1, \ldots, N, N$ being the total number of pixels in the image. The corresponding entropy $E$ is given in Eqn. 3.14.

\subsection{Exploring the NDVI with a Statistical Framework}

Having acquired the necessary tools, we now explore the NDVI in this section. We shall first obtain the NDVI via the PCA transformation. Then we shall consider the saturation problem of the NDVI and suggest a solution. We then demonstrate our solution to the saturation problem experimentally. Finally, we establish the relationship between the NDVI and the $R V I$.

\subsubsection{Estimating the PCA Parameters}

We use four meter resolution multispectral IKONOS satellite imagery. We have 5905 non-overlapping images each having $200 \times 200$ pixels in blue, green, red, and near-infrared bands; we only use red and near-infrared in this section. We estimate the PCA transformation matrix A based on these samples:

$$
\mathbf{A}=\left[\begin{array}{ll}
a_{11} & a_{12} \\
a_{21} & a_{22}
\end{array}\right]
$$

We construct the estimate as follows. We obtain a trial A for each image separately. We take $\mathbf{A}$ as a random matrix and obtain the mode matrix as described in Section 4.2.2. We assume it to be the true transformation matrix. We give the result as: 


$$
\mathbf{A}=\left[\begin{array}{cc}
-0.7071 & 0.7071 \\
0.7071 & 0.7071
\end{array}\right]
$$

By looking at $\mathbf{A}$, we see that each entry is equal to $\sqrt{2} / 2$ up to 14 th digit (we will explore this further) and this leads us to the NDVI formula.

To explore the PCA transformation, we give the scatter plot of a sample image with eigenvectors (multiplied by their eigenvalues) in Fig. 4.1. In this figure, we rescaled the eigenvectors for demonstration purposes. The corresponding eigenvalues are $\lambda_{1}=1.1408, \lambda_{2}=0.8592$. In this scatter plot, there are two main directions captured by the two eigenvectors with similar eigenvalues.

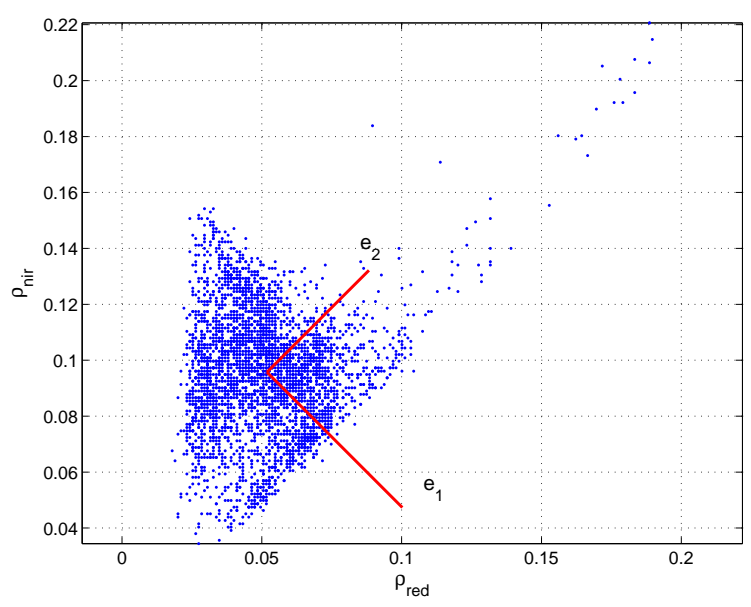

Figure 4.1: Scatter plot and eigenvectors for the NDVI

\subsubsection{Statistical Construction of the NDVI}

Let's start with $\mathbf{A}$. Uncorrelated variables $p c_{1}$ and $p c_{2}$ are calculated as follows:

$$
\left[\begin{array}{l}
p c_{1} \\
p c_{2}
\end{array}\right]=\mathbf{A} \times\left[\begin{array}{l}
\rho_{\text {red }} \\
\rho_{\text {nir }}
\end{array}\right]
$$


To observe this transformation graphically, we give Fig. 4.2. We see a $\pi / 4$ radian rotation from the $\rho_{\text {red }}, \rho_{\text {nir }}$ space to the $p c_{1}, p c_{2}$ space under this transformation.

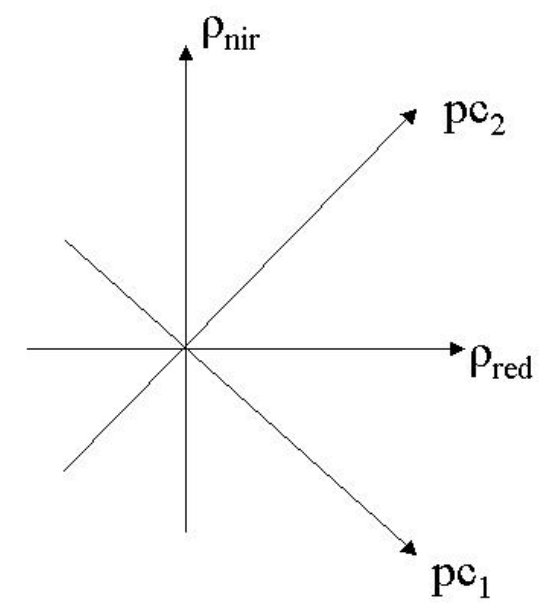

Figure 4.2: Rotation diagram for the PCA transformation

From the construction of the PCA, we know that $p c_{1}$ and $p c_{2}$ are statistically uncorrelated. We can define a slope $\alpha$ in the new decorrelated space as:

$$
\alpha=\frac{p c_{1}}{p c_{2}}
$$

If we rewrite the slope $\alpha$ in terms of $\rho_{\text {red }}$ and $\rho_{\text {nir }}$, we obtain:

$$
\alpha=\frac{\rho_{\text {nir }}-\rho_{\text {red }}}{\rho_{\text {nir }}+\rho_{\text {red }}}
$$

This slope definition directly corresponds to the definition of the NDVI given in Eqn. 4.4. This derivation proves that the NDVI is in fact the slope defined with respect to the decorrelated vector space. 


\subsubsection{Saturation Problem of the NDVI}

To this point we derived the NDVI by way of PCA and statistical parameter estimation. To our knowledge, this is the first attempt to put the NDVI onto a rigorous statistical footing. This derivation also suggests a solution to the saturation problems of the NDVI and the RVI. We first focus on the saturation problem of the $N D V I$; we develop the relationship between the $N D V I$ and the $R V I$ in the next section.

We begin by defining an angle $\phi$ corresponding to the slope value; which is of course the NDVI:

$$
\phi=\arctan (N D V I)
$$

To overcome the saturation problem, we normalize this angle by $\pi / 4$ to measure vegetation density as follows:

$$
\theta=\frac{4}{\pi} \phi=\frac{4}{\pi} \arctan (N D V I)
$$

As we know, $\arctan (\cdot)$ takes values in the $\pm \pi / 4$ range. Multiplying $\phi$ by $4 / \pi$ gives a measure normalized over \pm 1 .

Eqn. 4.18 indicates that $\theta$ is a nonlinear transformation of the NDVI. By applying this nonlinear transformation, we can offset the nonlinearity of the saturation. We will show by simulation and real image experiments how this method mitigates the saturation problem. 


\subsubsection{Experimental Results on the $N D V I$ and $\theta$ Measure}

In this section, we compare our normalized angle measure $\theta$ with the $N D V I$ with respect to linearity. For this reason, we evaluate their performances on a simulation and on real images.

We simulate the results of Bégué [148]. In his study, Bégué compared the NDVI and the leaf area index $(L A I)$ for various parameters and observed saturation curves similar to the one given in Fig. 4.3. In the simulation, we represent the vegetation density by the LAI. The NASA web site defines LAI as follows http:// earthobservatory .nasa.gov:81/libary/glossary ·php3:

"The area of foliage per unit area of ground. Conventionally this refers to the ratio of the area of the upper side of the leaves in a canopy projected onto a flat surface to the area of the surface under the canopy. Occasionally this has been used in reference to both sides of the leaves".

Here, we reconstruct one of the $N D V I$ vs. the $L A I$ curves of Bégué (represented in terms of percentages) and obtain the angle $\theta$ from it. We give the reconstructed curve and the angle $\theta$ in Fig. 4.3. Although the NDVI saturates as the vegetation density increases, the angle $\theta$ remains highly linear in this range. Certain surface materials (such as ice) produce negative values of $\theta$ (as for $N D V I$ ). Therefore $\theta \leq 0$ corresponds to zero vegetation here.

We also compare the $N D V I$ with the angle $\theta$ on real images. We pick four different images, representing various levels of vegetation density. To compare these measures, we give the color coded images (in these blue corresponds to the lowest vegetation value, and red corresponds to the highest). We also plotted the normalized histograms (pmfs) of the NDVI and $\theta$ for all images. 


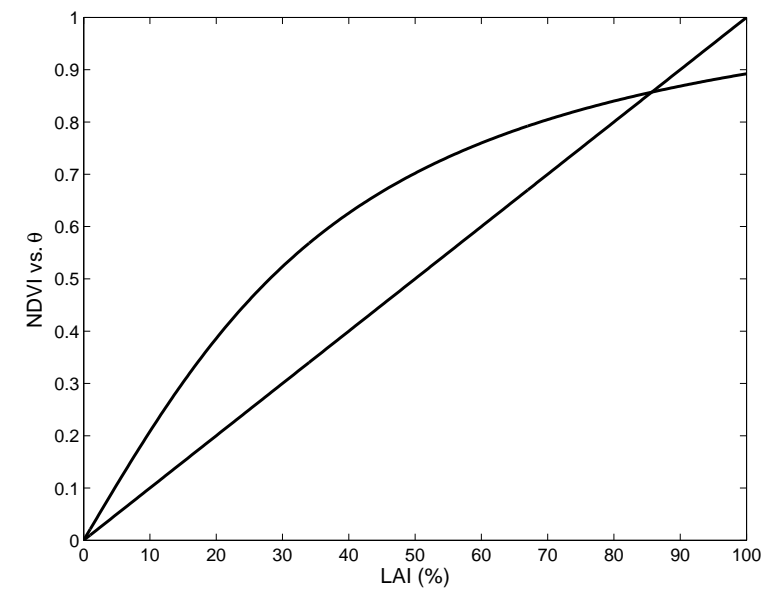

Figure 4.3: The $N D V I$ and angle $\theta$ vs. LAI

Let's start with the Maryland image given in Fig. 4.4. There is almost no vegetation in this region, except for two separate blocks (small green spaces). This is a typical downtown image with buildings and a road network. As expected, the NDVI and $\theta$ values are low. This is clearly seen from the histograms of two measures. So, our first conclusion is that when the vegetation density is low, both measures give similar responses.

Our next example is the New Mexico image given in Fig. 4.5. This image represents a typical residential region. The vegetation density is fairly high compared to the Maryland image. In this region, there are houses and a road network, in addition to the vegetation. Therefore, in vegetation measures we expect a higher contrast between vegetated and non-vegetated regions in this image. Dually, we expect a more uniform pmf for the better vegetation index. If we compare the normalized histograms (pmfs) of the $N D V I$ and $\theta$ for this image, we see that our measure has a 


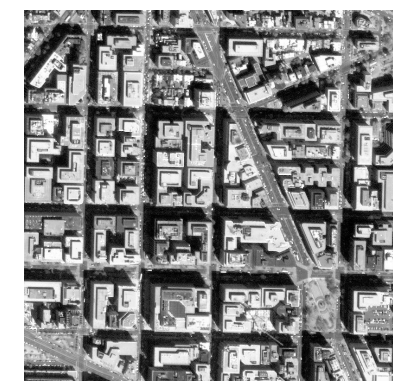

(a) The Maryland image

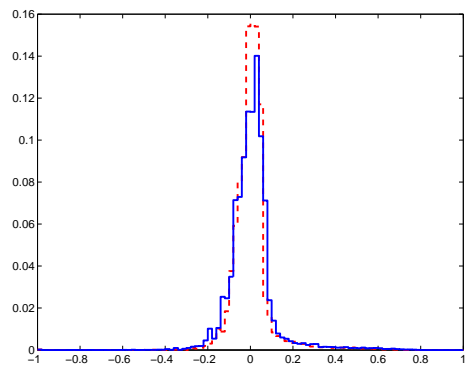

(d) Comparison of the histograms

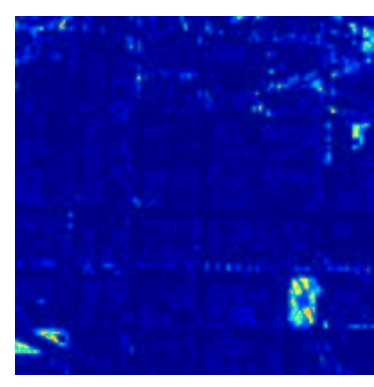

(b) The NDVI image

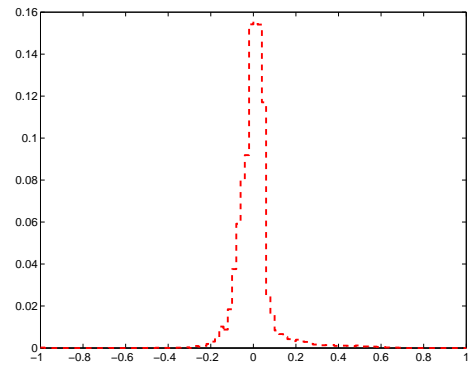

(e) Histogram for the NDVI image

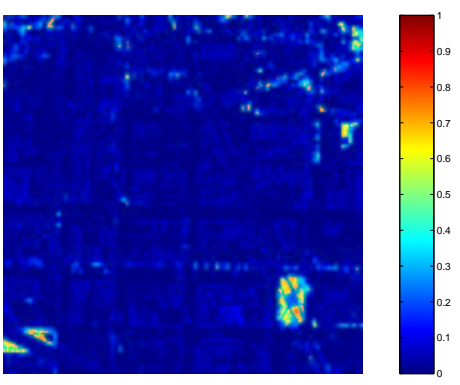

(c) $\theta$ image

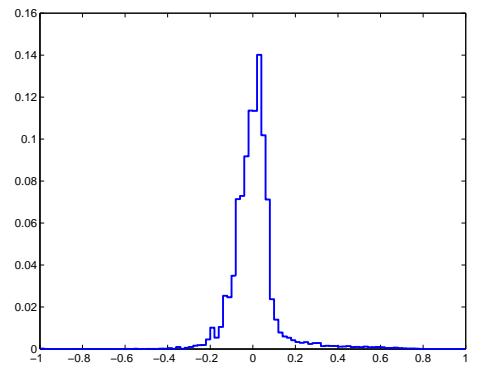

(f) Histogram for the $\theta$ image

Figure 4.4: The Maryland image, NDVI vs. $\theta$ representations

more uniform distribution. This is our second conclusion; as the vegetation density increases, our measure becomes more representative compared to the NDVI.

Next, we consider the Minnesota image given in Fig. 4.6. This image contains vegetated and bare soil regions. We picked this image to observe the contrast between these regions in the two vegetation measures. Similar to the New Mexico image, the normalized histogram obtained by our measure is more uniform compared to the histogram obtained from the $N D V I$ image. If we look at the region showing bare soil characteristics, we see that both measures give similar responses. So our measure 


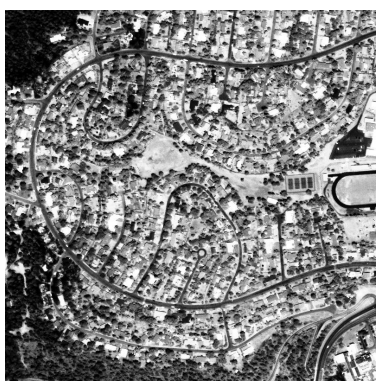

(a) The New Mexico image

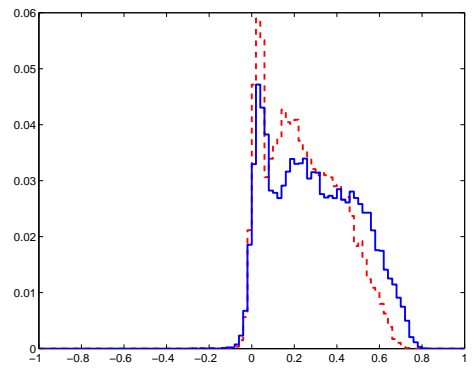

(d) Comparison of the histograms

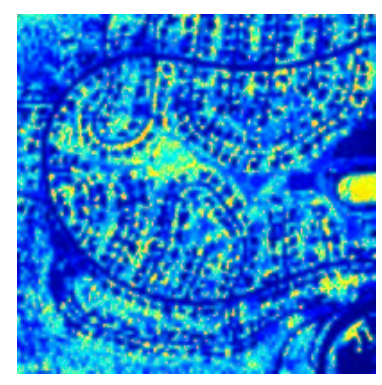

(b) The NDVI image

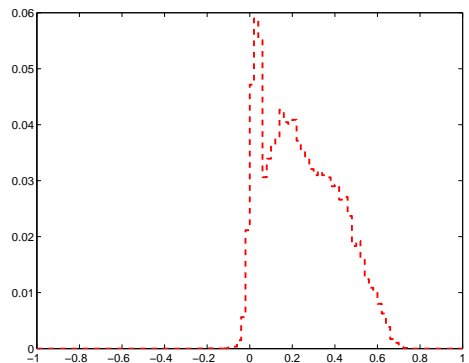

(e) Histogram for the NDVI image

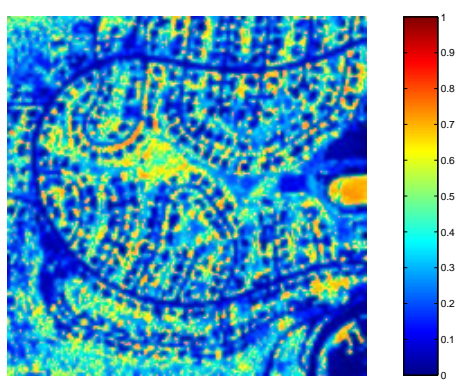

(c) $\theta$ image

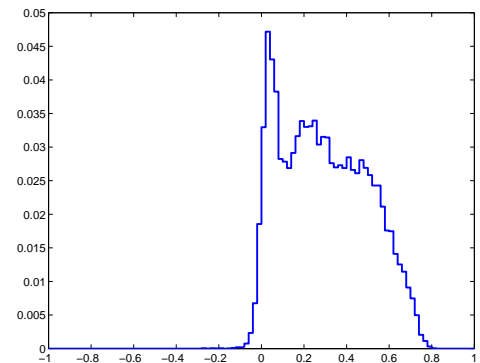

(f) Histogram for the $\theta$ image

Figure 4.5: The New Mexico image, $N D V I$ vs. $\theta$ representations 
$\theta$ not only responds more strongly to more vegetated regions, it also provides an appropriately weak response to non-vegetated regions.

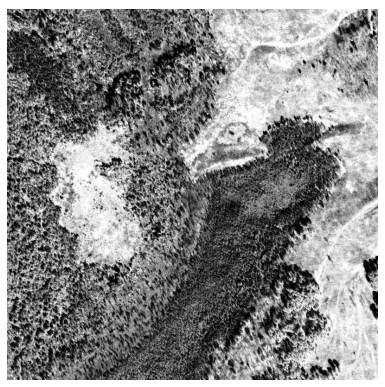

(a) The Minnesota image

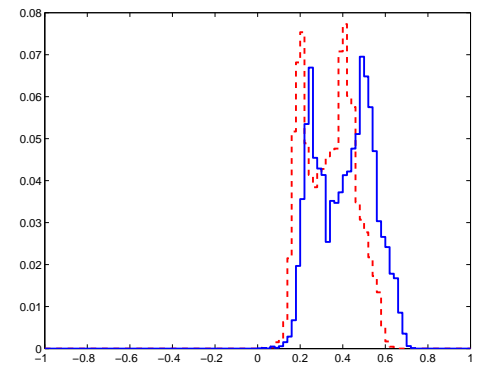

(d) Comparison of the histograms

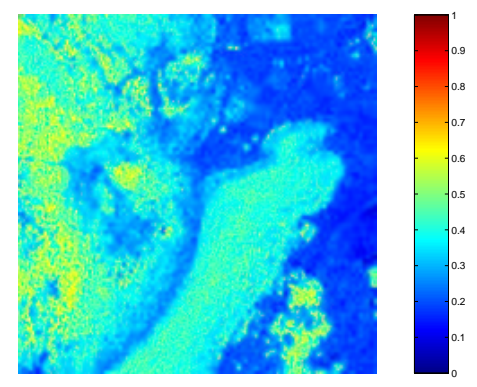

(b) The NDVI image

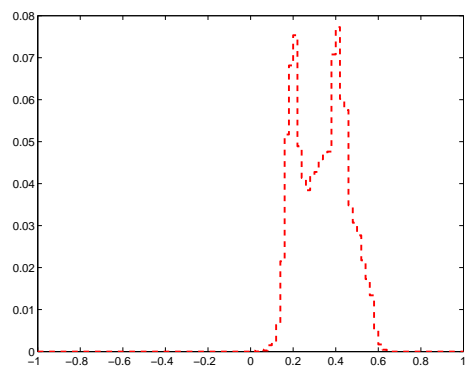

(e) Histogram for the NDVI image

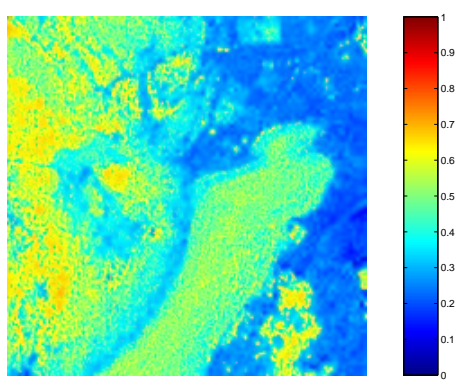

(c) $\theta$ image

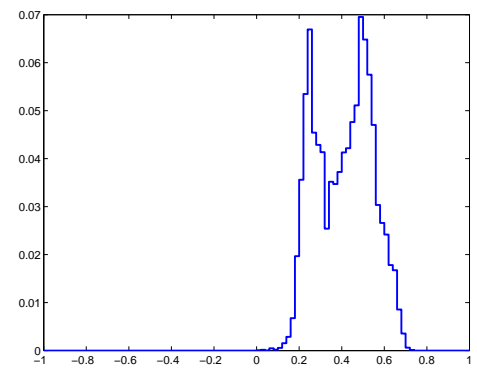

(f) Histogram for the $\theta$ image

Figure 4.6: The Minnesota image, $N D V I$ vs. $\theta$ representations

Our last example is the Oregon image given in Fig. 4.7. This is a densely forested region, one of the most vegetated regions in our data set. If we compare the normalized histograms of the two indices for this image, we see that $\theta$ gives a higher response to the forest region than the $N D V I$, as desired. This indicates that our measure $\theta$ is more linear than the NDVI; when the NDVI saturates our measure is still increasing in that range. 


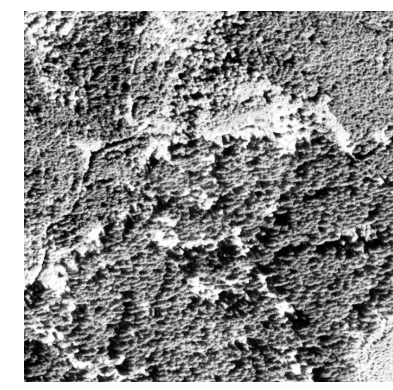

(a) The Oregon image

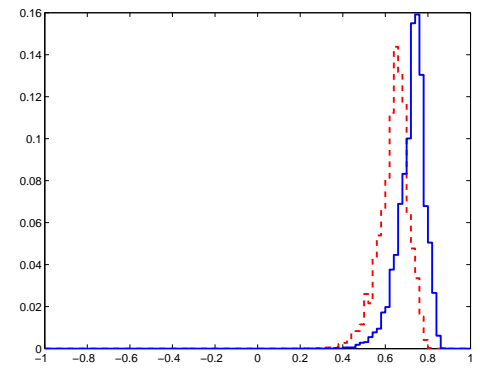

(d) Comparison of the histograms

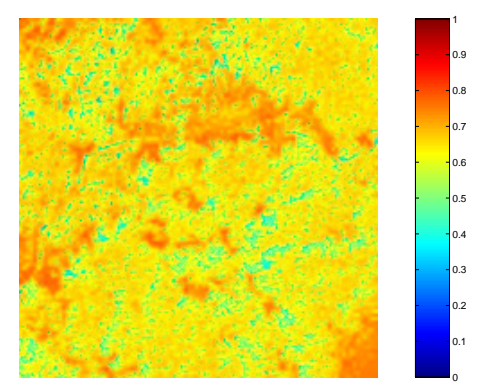

(b) The NDVI image

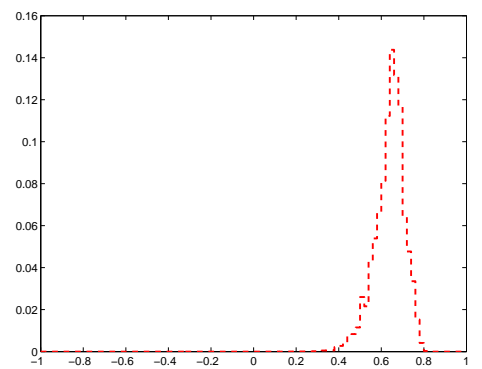

(e) Histogram for the NDVI image

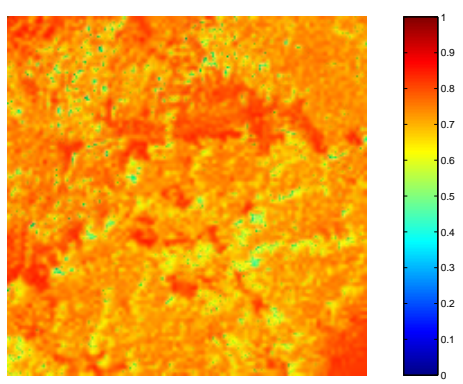

(c) $\theta$ image

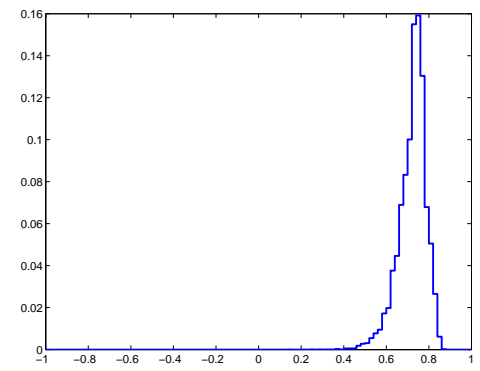

(f) Histogram for the $\theta$ image

Figure 4.7: The Oregon image, $N D V I$ vs. $\theta$ representations 
Finally, let's quantify the comparison of the $N D V I$ and $\theta$ measures. To achieve this goal, we apply three different tests. First, we take the Maryland image; we expect a low response to this image. Second, we take the Oregon image; we expect a high response to this image. Third, we take four new images given in Fig. 4.8 besides the New Mexico, and Minnesota images. All of these six images have high contrast between their vegetated and non-vegetated regions. Therefore, we calculate entropies on these images.

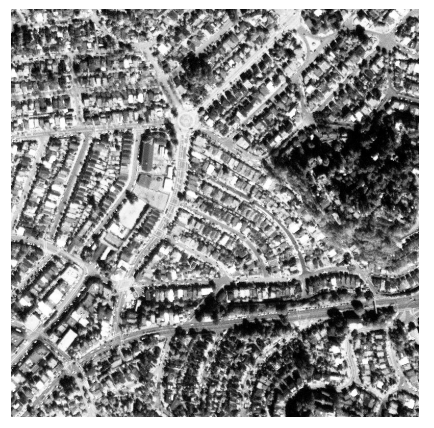

(a) The California 1 image

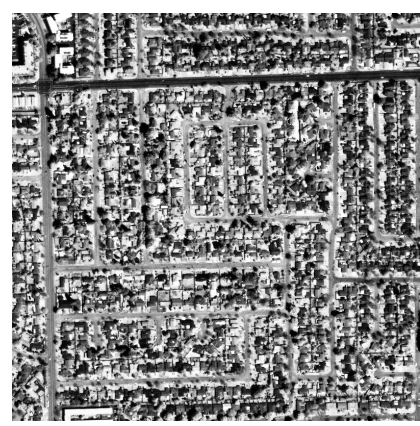

(c) The California 3 image

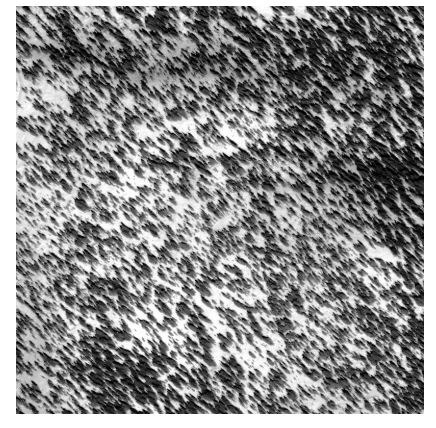

(b) The California 2 image

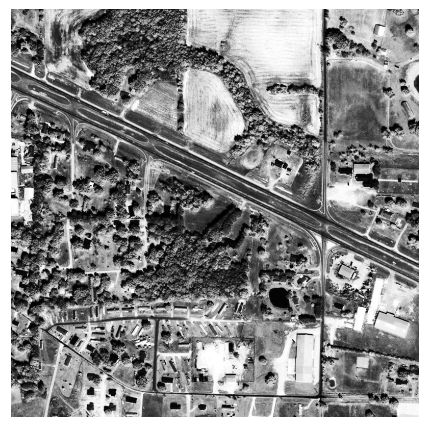

(d) The Maryland 2 image

Figure 4.8: Additional images for comparison based on entropy 
Let's consider the Maryland image. The sample mean for the NDVI is 0.0061 ; for $\theta$ it is 0.0072 . So, both measures give similar low responses. For the Oregon image, sample means are 0.6387 and 0.7217 for the $N D V I$ and $\theta$ images respectively. On this densely vegetated image, our measure outperformed the NDVI.

Finally, let's compare the entropies of both measures on the six high contrast images. We label images as $i m g_{i}$ for $i=1,2, \ldots, 6$ in the order of California 1, California 2, New Mexico, Minnesota, California 3, and Maryland 2. The corresponding entropies are tabulated in Table 4.1.

\begin{tabular}{|l|c|c|c|c|c|c|c|}
\hline & $i m g_{1}$ & $i m g_{2}$ & $i m g_{3}$ & $i m g_{4}$ & $i m g_{5}$ & $i m g_{6}$ & Mean Value \\
\hline$E_{N D V I}$ & 4.9145 & 4.8242 & 4.9825 & 4.4330 & 4.9156 & 5.1361 & 4.8677 \\
\hline$E_{\theta}$ & 5.1858 & 5.0849 & 5.2148 & 4.6091 & 5.1755 & 5.2844 & 5.0924 \\
\hline
\end{tabular}

Table 4.1: Comparison of the entropies for the six images for the NDVI and $\theta$

On all six images, our measure obtained higher entropy values than did the NDVI. This is also evident from the mean values of these entropies. Therefore, the entropy test underscores the improved linearity of our measure with respect to the NDVI.

\subsubsection{The Relationship Between the NDVI and RVI}

We now return our attention to the PCA transformation matrix $\mathbf{A}$ given in Eqn. 4.13. As we recall, the entries of this matrix equal $\sqrt{2} / 2$ to 14 th digit. This points to a relationship between the ratio vegetation index $(R V I)$ and the NDVI. Our aim here is twofold. First, we prove that the NDVI and the RVI capture exactly the 
same information. That is, they calculate the same slope in two different representations of the same space, one representation being a rotated version of the other. Second, the angle defined above also applies to the $R V I$, after a shift of $\pi / 4$ radians.

Let's start with the definition of axis rotation by a clockwise angle $\beta$. Assume that our initial coordinates are $\rho_{\text {red }}$ and $\rho_{\text {nir }}$. If we want to obtain the rotated coordinate axes $\hat{x}, \hat{y}$ from them, we apply the following transformation:

$$
\left[\begin{array}{l}
\hat{x} \\
\hat{y}
\end{array}\right]=\left[\begin{array}{cc}
\cos (\beta) & -\sin (\beta) \\
\sin (\beta) & \cos (\beta)
\end{array}\right] \times\left[\begin{array}{c}
\rho_{\text {red }} \\
\rho_{\text {nir }}
\end{array}\right]
$$

We can see the effect of this axis rotation graphically in Fig. 4.9.

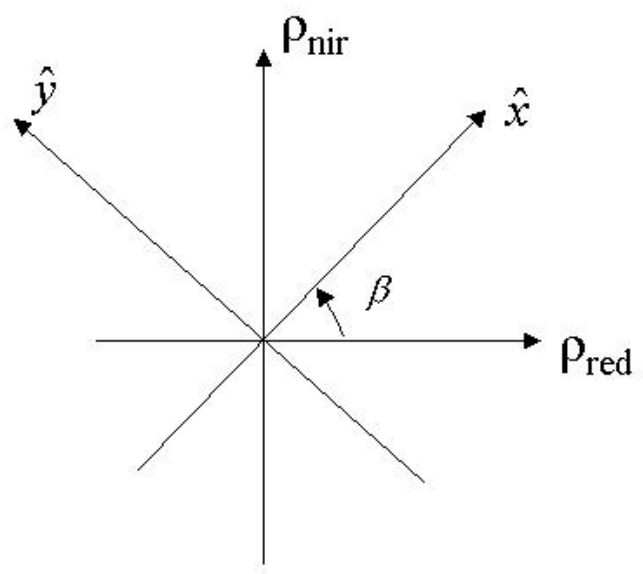

Figure 4.9: Axis rotation by $\beta$ radians

Let's take $\beta=-\pi / 4$; then we obtain our new coordinates as follows:

$$
\left[\begin{array}{l}
\hat{x} \\
\hat{y}
\end{array}\right]=\frac{1}{\sqrt{2}}\left[\begin{array}{l}
\rho_{\text {nir }}+\rho_{\text {red }} \\
\rho_{\text {nir }}-\rho_{\text {red }}
\end{array}\right]
$$

If we take the ratio of the new coordinates, we obtain the NDVI as follows: 


$$
N D V I=\frac{\hat{y}}{\hat{x}}
$$

Recall, $R V I$ was just the ratio of the original coordinates $\left(\rho_{\text {nir }}, \rho_{\text {red }}\right)$ as in Eqn. 4.1. Here we have that the $N D V I$ is the slope in a rotated coordinate system. Recalling $\theta$ as defined in Eqn. 4.17, we see that this rotation relationship indicates that we can obtain a similar measure from the $R V I$. This angle will simply be $\pi / 4$ radians displaced from that obtained from the NDVI. However, there is little reason to do so because, again, if $\rho_{\text {red }}$ is small the measure will be unstable. Our measure $\theta$ does not suffer from this effect.

\subsection{New Vegetation Indices Based on the Statistical Frame- work}

Having such a powerful statistical framework to analyze the NDVI suggests that more vegetation indices may be defined. As we mentioned, the NDVI only uses the red and near-infrared bands. To these, we add the blue and green bands to observe whether we can obtain more information from them.

We start by adding the blue band to the red and near-infrared to compute a new transformation. We then replace the blue band with the green. Finally, we consider all four bands in the transformation to determine whether a better vegetation index

results. All measures will be normalized between \pm 1 as is $\theta$. In this section, we compare each new index with our measure $\theta$ on real images. 


\subsubsection{A Vegetation Index Using Blue, Red, and Near - In- frared Bands}

To reduce atmospheric effects, most authors included the blue band in their vegetation indices. To see the influence of the blue band, we introduce it in our framework alongside the red and near-infrared bands. We estimate new transformation matrix, and apply the PCA transformation to this three dimensional space as we did for the NDVI derivation. Finally, we obtain the transformed space as:

$$
\left[\begin{array}{l}
p c_{21} \\
p c_{22} \\
p c_{23}
\end{array}\right]=\left[\begin{array}{ccc}
-0.5960 & -0.6033 & -0.5299 \\
0.4167 & 0.3317 & -0.8464 \\
0.6864 & -0.7253 & 0.0537
\end{array}\right] \times\left[\begin{array}{c}
\rho_{\text {blue }} \\
\rho_{\text {red }} \\
\rho_{\text {nir }}
\end{array}\right]
$$

To explore the PCA transformation for this space, we give the scatter plot of a sample image with eigenvectors (multiplied by their eigenvalues) in Fig. 4.10. We rescaled the eigenvectors for demonstration purposes. The corresponding eigenvalues are $\lambda_{1}=1.9734, \lambda_{2}=0.9741$, and $\lambda_{3}=0.0525$. Since two eigenvalues are much greater than the third, we conclude that the data scatter primarily over a 2D manifold in $\Re^{3}$. Thus, even in $3 \mathrm{D}$, this is largely a $2 \mathrm{D}$ problem. Inspired by the $N D V I$ (and experimentally checking its performance compared to other combinations), we pick the angle between the second eigenvector and the sum vector as a new vegetation index in this space:

$$
\theta_{2}=\frac{4}{\pi} \arctan \frac{-p c_{22}}{\sqrt{p c_{21}^{2}+p c_{22}^{2}+p c_{23}^{2}}}
$$

This index is similar to $\theta$ formulation we obtained from the NDVI. The ratio inside the arctangent is in fact a slope in the transformed space. By using the angle representation instead of this slope, we again obtain a more linear vegetation index. 


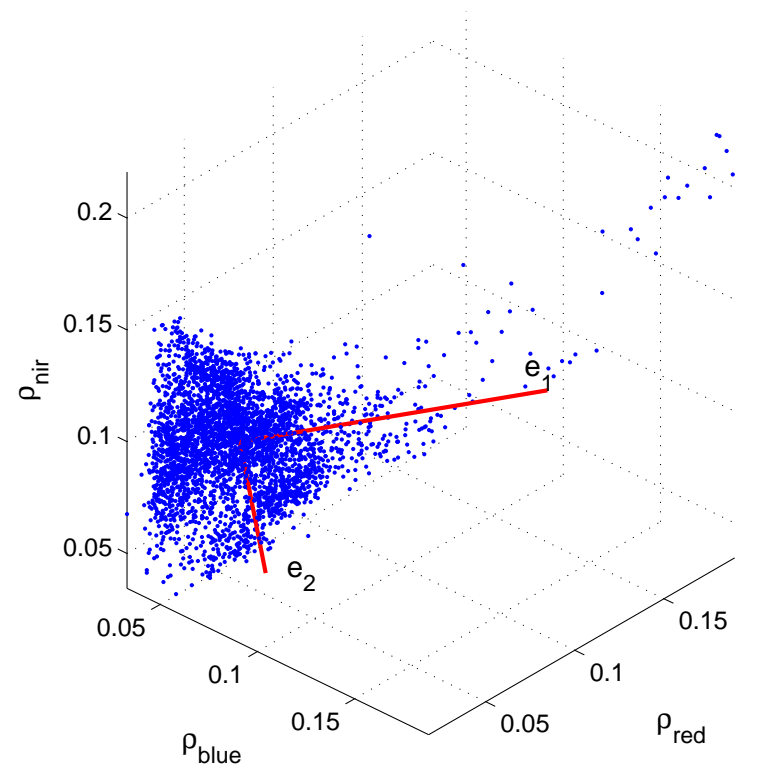

Figure 4.10: Scatter plot and eigenvectors for the blue, red, and near-infrared bands

\subsubsection{A Vegetation Index Using Green, Red, and Near - In- frared Bands}

We replace the blue band with the green in this section. For these bands we obtain a transformed space as:

$$
\left[\begin{array}{l}
p c_{31} \\
p c_{32} \\
p c_{33}
\end{array}\right]=\left[\begin{array}{ccc}
-0.5986 & -0.6000 & -0.5308 \\
0.3836 & 0.3670 & -0.8474 \\
0.7032 & -0.7109 & -0.0105
\end{array}\right] \times\left[\begin{array}{c}
\rho_{\text {green }} \\
\rho_{\text {red }} \\
\rho_{\text {nir }}
\end{array}\right]
$$

To explore the PCA transformation for this space, we give the scatter plot of a sample image with eigenvectors (multiplied by their eigenvalues) in Fig. 4.11. We again rescale the eigenvectors for demonstration purposes. The corresponding eigenvalues are $\lambda_{1}=2.0226, \lambda_{2}=0.9511$, and $\lambda_{3}=0.0263$ and similar observations regarding the scatter (2D manifold in $\Re^{3}$ ) apply. One difference in this space is that the difference between the first and second eigenvalues is higher. The last eigenvalue 
is also lower compared to the previous space. We again pick the angle between the second eigenvector and the sum vector as a new index in this space:

$$
\theta_{3}=\frac{4}{\pi} \arctan \frac{-p c_{32}}{\sqrt{p c_{31}^{2}+p c_{32}^{2}+p c_{33}^{2}}}
$$

This index has characteristics similar to those of $\theta_{2}$.

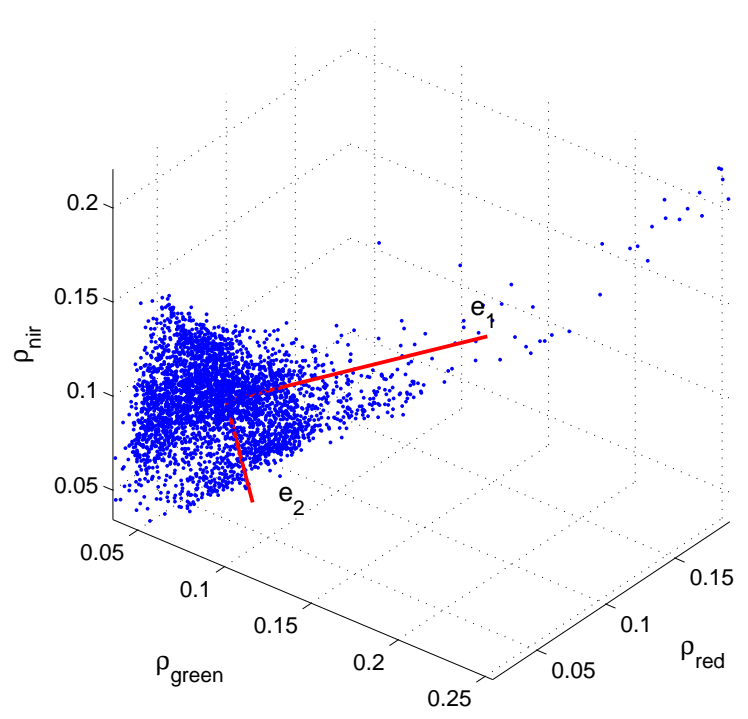

Figure 4.11: Scatter plot and eigenvectors for the green, red, and near-infrared bands

\subsubsection{A Vegetation Index Using All Four Bands}

Finally, we use all the bands to obtain yet another vegetation index. By using blue, green, red, and near-infrared bands we hope to extract all the information our multispectral images can provide. As we apply the statistical framework for the NDVI to this space, we obtain a transformed space as: 


$$
\left[\begin{array}{l}
p c_{41} \\
p c_{42} \\
p c_{43} \\
p c_{44}
\end{array}\right]=\left[\begin{array}{cccc}
-0.5190 & -0.5258 & -0.5253 & -0.4221 \\
0.3091 & 0.2110 & 0.2093 & -0.9034 \\
0.7034 & -0.0425 & -0.7063 & 0.0671 \\
0.3746 & -0.8229 & 0.4259 & 0.0346
\end{array}\right] \times\left[\begin{array}{c}
\rho_{\text {blue }} \\
\rho_{\text {green }} \\
\rho_{\text {red }} \\
\rho_{\text {nir }}
\end{array}\right]
$$

For this four dimensional space we are unable to provide the scatter plot. However, the eigenvalues are $\lambda_{1}=2.9636, \lambda_{2}=0.9756, \lambda_{3}=0.0538$, and $\lambda_{3}=0.0070$. There are again two major principal components, with fairly high eigenvalues. The difference between the first and the second eigenvalue has increased compared to the three dimensional transformations. The third eigenvalue has similar values to the three dimensional transformations. The fourth eigenvector is almost insignificant based on its eigenvalue. For a new vegetation index, we again reach a similar formula as we did for the three dimensional case as follows:

$$
\theta_{4}=\frac{4}{\pi} \arctan \frac{-p c_{42}}{\sqrt{p c_{41}^{2}+p c_{42}^{2}+p c_{43}^{2}+p c_{44}^{2}}}
$$

This index also has characteristics similar to those of $\theta_{2}$ and $\theta_{3}$.

\subsection{Comparing the Vegetation Indices}

Up to now, we introduced a linearized vegetation index $\theta$ from the $N D V I$ and three new vegetation indices $\theta_{2}, \theta_{3}$, and $\theta_{4}$ from the $3 \mathrm{D}$ and $4 \mathrm{D}$ transformed spaces. Here, we compare these four indices using three different criteria. The first criterion is based on the visual comparison and dynamic range by their normalized histograms. The second criterion is based on the entropy of these indices on six high contrast images as in Section 4.3.4. The third criterion is based on the computational cost. 


\subsubsection{Comparison by the First Criterion (Visual Comparison and Dynamic Range)}

In this section we compare these indices on the Maryland, New Mexico, Minnesota, and the Oregon images (Figs. 4.4, 4.5, 4.6, and 4.7) to see which measures vegetation density best. As we mentioned previously, we chose and sorted these images based on their vegetation densities. We give the color coded responses (blue corresponds to the lowest vegetation value, and red corresponds to the highest) of all our vegetation indices to these images in Fig. 4.12. In this figure, responses to the Maryland, New Mexico, Minnesota, and the Oregon images are represented in the first, second, third, and fourth rows respectively.

We can see that all of them perform fairly well in measuring vegetation density as given in Fig. 4.12. The indices not using the blue band, $\theta$ and $\theta_{3}$, do not respond well to shadows in the Maryland and the Oregon images. That is, vegetation in shadow confuses these indices to some degree. The remaining indices, $\theta_{2}$ and $\theta_{4}$, can also be used to detect shadows (again in the Maryland and Oregon images) by virtue of their low responses to them. Their response to shadows increase the contrast between the vegetated and non-vegetated (especially shadow) regions. So our first conclusion is that, if we require precise vegetation, we should use an index having the blue band in its formulation $\left(\theta_{2}\right.$ or $\left.\theta_{4}\right)$.

To compare the dynamic range of these four vegetation indices, we next obtain the normalized histograms on the four images. In Fig. 4.13 we give these histograms; in which the solid curve represents $\theta$, the dotted curve represents $\theta_{2}$, the dashed and dotted curve represents $\theta_{3}$, and the dashed curve represents $\theta_{4}$. 


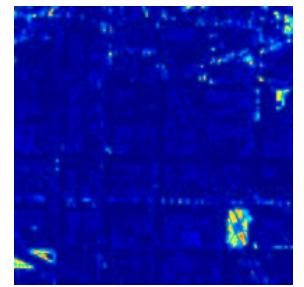

(a) $\theta$

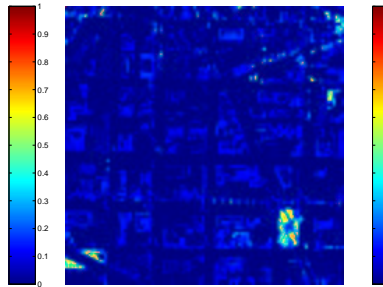

(b) $\theta_{2}$

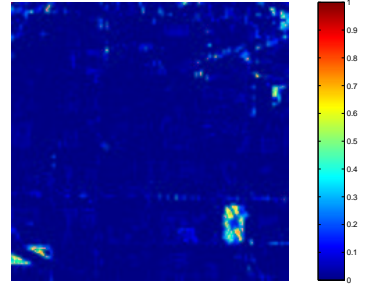

(c) $\theta_{3}$

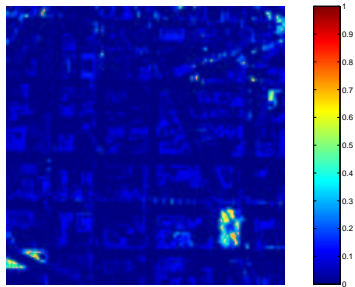

(d) $\theta_{4}$

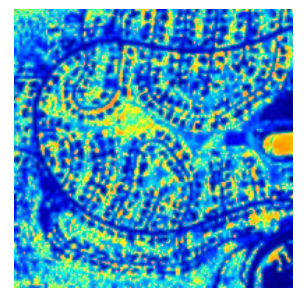

(e) $\theta$

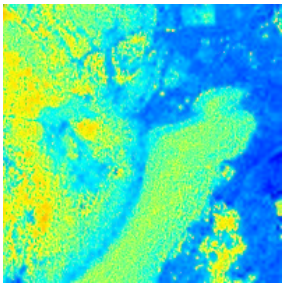

(i) $\theta$

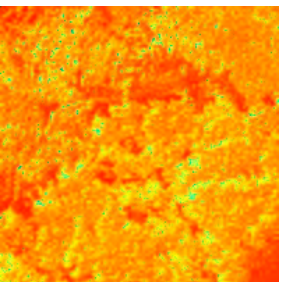

(m) $\theta$

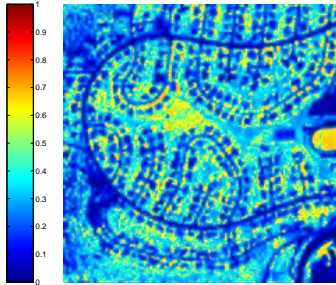

(f) $\theta_{2}$

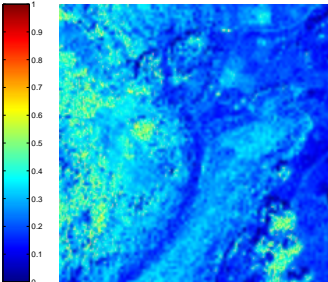

(j) $\theta_{2}$

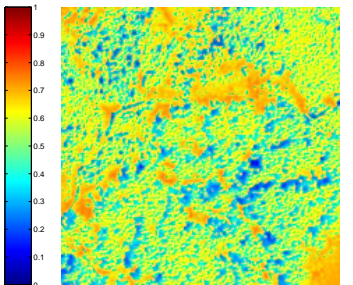

(n) $\theta_{2}$

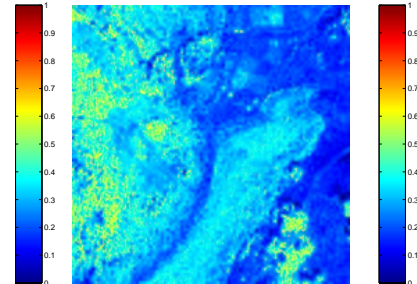

(k) $\theta_{3}$

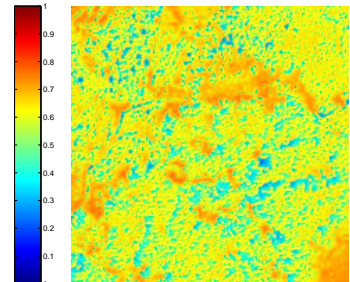

(o) $\theta_{3}$

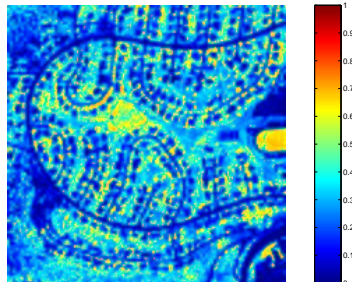

(h) $\theta_{4}$

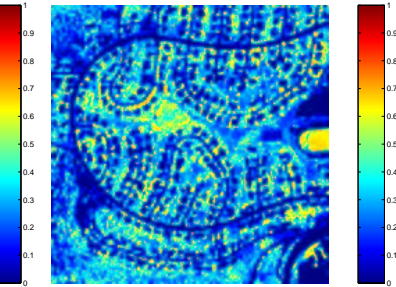

(g) $\theta_{3}$

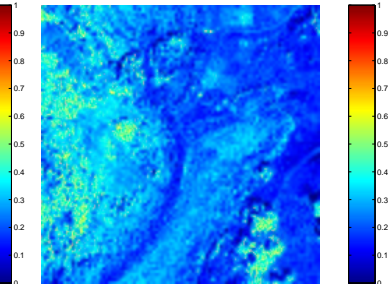

(l) $\theta_{4}$

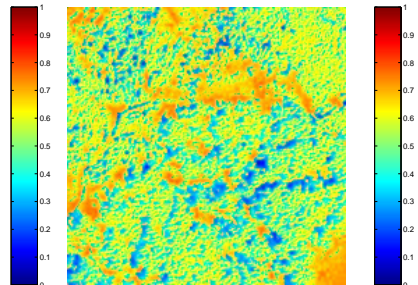

(p) $\theta_{4}$

Figure 4.12: Comparison of vegetation indices on real images 


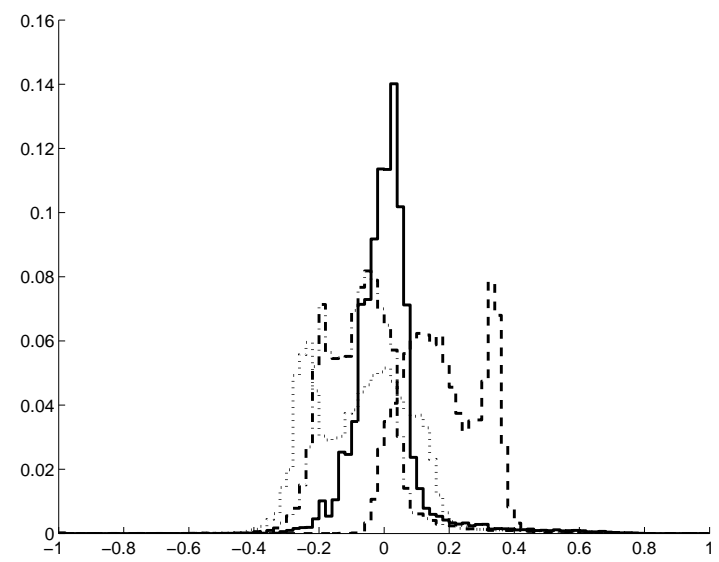

(a) Histograms for the Maryland image

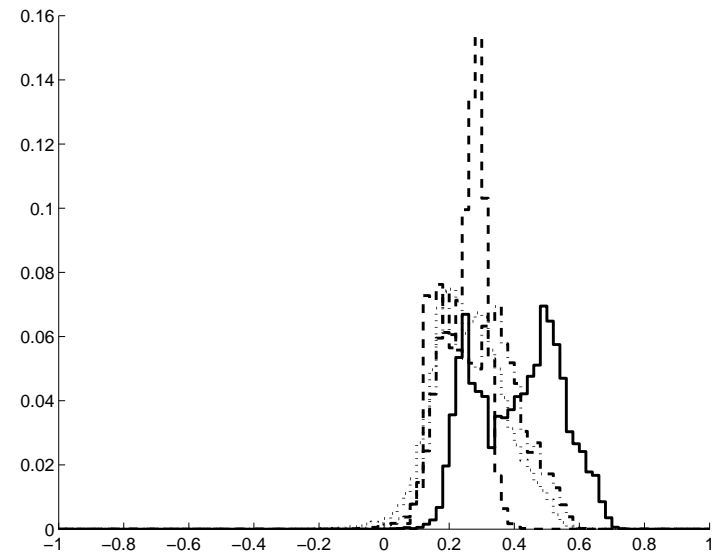

(c) Histograms for the Minnesota image

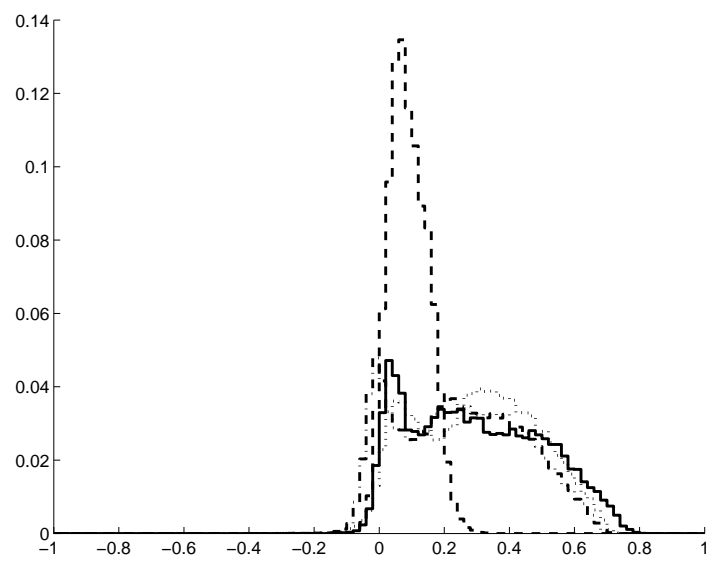

(b) Histograms for the New Mexico image

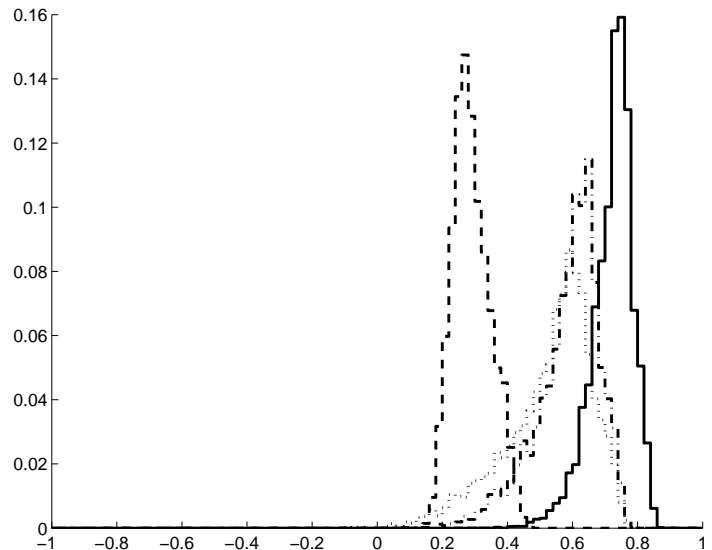

(d) Histograms for the Oregon image

Figure 4.13: Comparison of histograms for the four vegetation indices to compare their dynamic ranges. ( solid $-\theta$, dot $-\theta_{2}$, dash and dot $-\theta_{3}$, dash $-\theta_{4}$ ) 
If we compare these histogram values, we see that $\theta_{4}$ has the lowest dynamic range except the Maryland image (least vegetation). In the two high contrast images (New Mexico, and Minnesota) $\theta$ has the highest dynamic range. For these images $\theta_{2}$ and $\theta_{3}$ performed similarly. For the Oregon image, $\theta$ has a lower dynamic range, but the important point is that this range is close to one as desired. Again for this image $\theta_{2}$, and $\theta_{3}$ performed similarly. $\theta_{4}$ performed poorly in giving the lowest response to this image.

Next, let's focus on the two extremes (Maryland and Oregon) to compare responses of the four vegetation indices by their sample means, $\mu$. As we recall, the Maryland image has almost no vegetation, so the best index response to this image should be a value near zero. The Oregon image has the highest vegetation density, so a good index should have a strong response to it. We give these comparisons in Table 4.2.

\begin{tabular}{|l|c|c|c|c|}
\hline & $\mu_{\theta}$ & $\mu_{\theta_{2}}$ & $\mu_{\theta_{3}}$ & $\mu_{\theta_{4}}$ \\
\hline The Maryland image & 0.0072 & -0.0658 & -0.0743 & 0.1835 \\
\hline The Oregon image & 0.7217 & 0.5286 & 0.5861 & 0.2903 \\
\hline
\end{tabular}

Table 4.2: Comparison of the four vegetation indices on the Maryland and Oregon images

As seen from the first row of Table 4.2 , all indices except $\theta_{4}$ performed fairly well on responding the Maryland image. For the Oregon image (given in the second row of Table 4.2) $\theta$ outperformed all other indices by having the highest value. $\theta_{3}$ and $\theta_{2}$ have the second and third highest values. Again $\theta_{4}$ performed poorly. Based on this criterion, we can sort these indices as $\theta, \theta_{3}, \theta_{2}$, and $\theta_{4}$ from best to worst. 


\subsubsection{Comparison by the Second Criterion (Entropy on High Contrast Images)}

Next, let's compare these four indices based on the entropy of them on six high contrast images as in Section 4.3.4. We give the results in Table 4.3.

\begin{tabular}{|l|c|c|c|c|c|c|c|}
\hline & $i m g_{1}$ & $i m g_{2}$ & $i m g_{3}$ & $i m g_{4}$ & $i m g_{5}$ & $i m g_{6}$ & Mean Value \\
\hline$E_{\theta}$ & 5.1858 & 5.0849 & 5.2148 & 4.6091 & 5.1755 & 5.2844 & 5.0924 \\
\hline$E_{\theta_{2}}$ & 5.2388 & 4.9506 & 5.1381 & 4.4696 & 5.2649 & 5.0725 & 5.0224 \\
\hline$E_{\theta_{3}}$ & 5.1323 & 4.9629 & 5.1487 & 4.4801 & 5.1696 & 5.2083 & 5.0170 \\
\hline$E_{\theta_{4}}$ & 4.0726 & 3.0714 & 3.5988 & 3.6012 & 3.6369 & 3.9555 & 3.6561 \\
\hline
\end{tabular}

Table 4.3: Comparison of the entropies on six images for the four vegetation indices

This table indicates that $\theta$ has the highest average entropy over the images considered. $\theta_{2}$ and $\theta_{3}$ has similar entropies in the second and the third place. Finally, $\theta_{4}$ has the lowest average entropy. Based on this criterion, we can sort these indices as $\theta, \theta_{2}, \theta_{3}$, and $\theta_{4}$ from best to worst.

\subsubsection{Comparison by the Third Criterion (Computational Cost)}

There are also computation costs assigned to these indices. Our first measure, $\theta$ needs only the red and near-infrared bands. Indices $\theta_{2}$ and $\theta_{3}$ need three bands, while $\theta_{4}$ needs four bands to operate. As we mentioned, if atmospheric effects are considered $\theta_{2}$ seems to be the best measure since it uses the blue band and it performs about as well as $\theta$.

Based on our criteria $\theta$ and $\theta_{2}$ are the most promising two indices to measure vegetation. The final selection of a "best" measure will depend to some extent on 
the application. To summarize, we give these two most promising indices in terms of $\rho_{\text {blue }}, \rho_{\text {red }}$, and $\rho_{\text {nir }}$ below:

$$
\begin{gathered}
\theta=\frac{4}{\pi} \arctan \left(\frac{\rho_{\text {nir }}-\rho_{\text {red }}}{\rho_{\text {nir }}+\rho_{\text {red }}}\right) \\
\theta_{2}=\frac{4}{\pi} \arctan \frac{-0.4167 \rho_{\text {blue }}-0.3317 \rho_{\text {red }}+0.8464 \rho_{\text {nir }}}{\sqrt{\rho_{\text {blue }}^{2}+\rho_{\text {red }}^{2}+\rho_{\text {nir }}^{2}}}
\end{gathered}
$$

\subsection{Shadow-Water Indices}

The next group of indices we introduce within the same statistical framework are the shadow-water indices (SWI). We were unable to find a focused work on water detection on satellite imagery. However, the closest matches in the literature are [167-169]. Shadows (clouds) also have photometric characteristics similar to water. Shadow (cloud) detection studies include [170-176].

Simpson and Stitt [173] worked on cloud shadow detection in AVHRR (advanced very high resolution radiometer) imagery. To detect shadows, they used geometric and optical constraints on a pixel basis in multispectral images. They discarded water regions to focus on clouds.

In the IKONOS spectrum, water shows an increasing response curve until the blue band, it reaches the maximum in this region and then decreases monotonically to the near infrared [177]. So a representative shadow water index should be composed of high blue values first. Ideally it should also consider the green and red bands, but the green band also responds strongly to vegetation and this impairs the shadow or water observation. Hence, the index should include blue and red bands at least. To obtain

such an index, we applied the same framework we used for the $N D V I$ derivation using 
principal components analysis with the blue, red, and near infrared bands. Based on the combinatorial search (and trying to maximize blue and red band coefficients) we obtain the best performing shadow-water index for each dimension.

We start with a two dimensional space. Since we plan to have the blue band in a candidate SWI, we replace the red band with the blue in the NDVI formulation. We obtain our first index as:

$$
\gamma_{1}=\frac{4}{\pi} \arctan \left(\frac{\rho_{\text {nir }}-\rho_{\text {blue }}}{\rho_{\text {nir }}+\rho_{\text {blue }}}\right)
$$

For the three and four dimensional spaces, we observe that the third principal component has the shadow information in all the transformed spaces. Shadows and water are relatively uncommon in our training data, so the mean-square energy in that component will be less than two - but not least of four - components. We give the corresponding shadow-water indices $\gamma_{2}, \gamma_{3}$, and $\gamma_{4}$ obtained from the transformation spaces given in Eqns. 4.22, 4.24, and 4.26 as:

$$
\begin{gathered}
\gamma_{2}=\frac{4}{\pi} \arctan \frac{-p c_{23}}{\sqrt{p c_{21}^{2}+p c_{22}^{2}+p c_{23}^{2}}} \\
\gamma_{3}=\frac{4}{\pi} \arctan \frac{-p c_{33}}{\sqrt{p c_{31}^{2}+p c_{32}^{2}+p c_{33}^{2}}} \\
\gamma_{4}=\frac{4}{\pi} \arctan \frac{-p c_{43}}{\sqrt{p c_{41}^{2}+p c_{42}^{2}+p c_{43}^{2}+p c_{44}^{2}}}
\end{gathered}
$$




\subsection{Comparing the Shadow-Water Indices}

Similar to the Section 4.5, we compare the four SWI $\gamma_{1}, \gamma_{2}, \gamma_{3}$, and $\gamma_{4}$ in this section. Here, we only use the first two criteria, since computation costs for the SWI are the same as the corresponding vegetation indices.

\subsubsection{Comparison by the First Criterion (Visual Comparison and Dynamic Range)}

To compare these SWI on real images, we pick four regions from Maryland, Oregon, Indiana, and Florida given in Fig. 4.14. The Maryland image (again) represents a typical urban area and contains shadows cast by tall buildings. The Oregon image is of a forested region with tree shadows. There is a small lake in the middle of the Indiana image. Finally, the Florida image is of the ocean. We give the color coded responses (blue corresponds to the lowest value, and red corresponds to the highest) of the four SWI with respect to each image in Fig. 4.15.

Our first observation is that $\gamma_{3}$, which uses the green, red, and near-infrared bands, performs poorly in responding to shadows compared to $\gamma_{1}, \gamma_{2}$, and $\gamma_{4}$. This is expected since it does not have the blue band in its formation, and we know that the blue band bears the shadow and water information. Nevertheless, $\gamma_{3}$ represents the best one can expect should only green, red, and near-infrared bands be available.

If we compare $\gamma_{2}$ with $\gamma_{4}$ we conclude that $\gamma_{2}$ outperforms $\gamma_{4}$ in representing the shadow and water density, while using only the blue, red, and near-infrared bands. So we need just three bands instead of four (as for the $\gamma_{4}$ ) to compute it.

Finally, if we compare $\gamma_{1}$ with $\gamma_{2}$, for the Oregon image $\gamma_{1}$ cannot detect shadows on the trees. However, it performs better on the Indiana and Florida images. The 


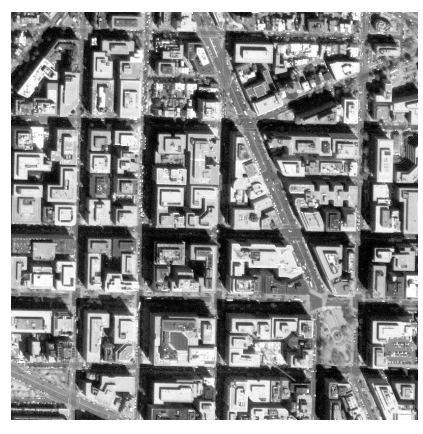

(a) The Maryland image

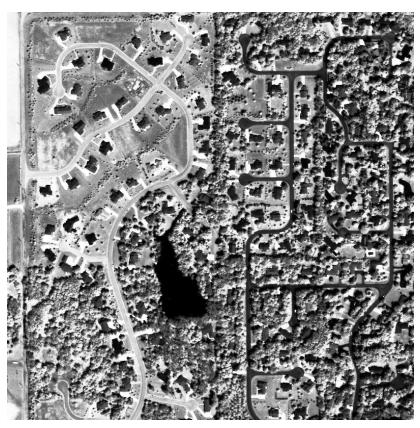

(c) The Indiana image

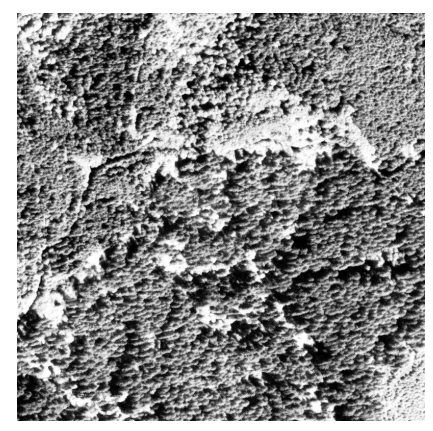

(b) The Oregon image

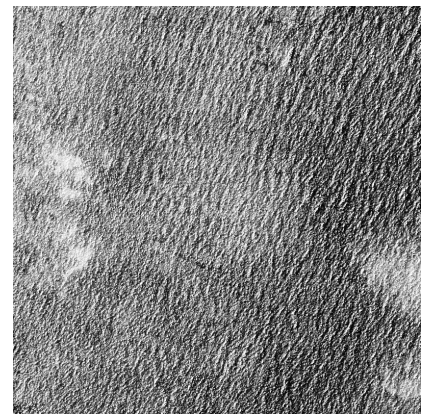

(d) The Florida image

Figure 4.14: Sample images to compare the four SWI 


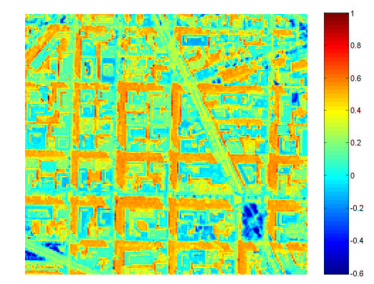

(a) $\gamma_{1}$

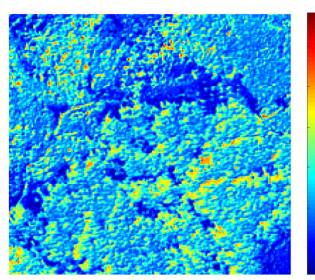

(e) $\gamma_{1}$

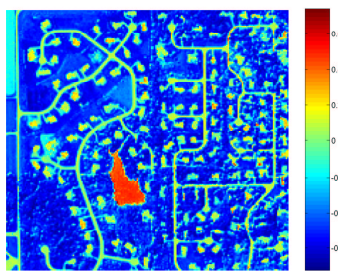

(i) $\gamma_{1}$

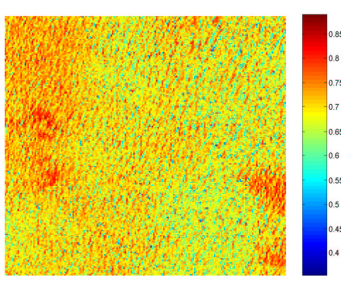

(m) $\gamma_{1}$

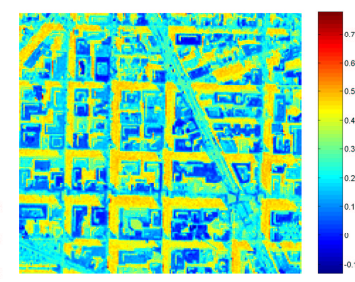

(b) $\gamma_{2}$

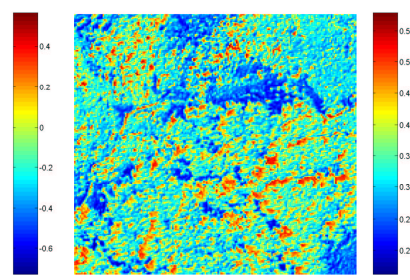

(f) $\gamma_{2}$

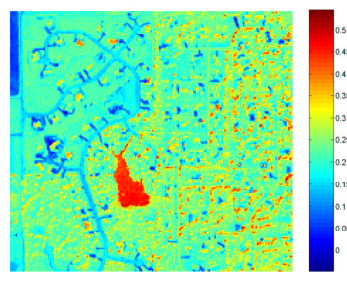

(j) $\gamma_{2}$

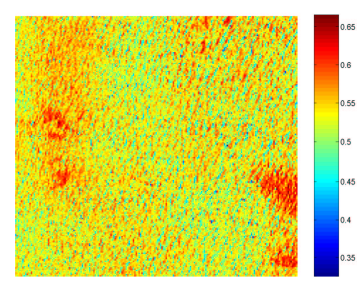

(n) $\gamma_{2}$

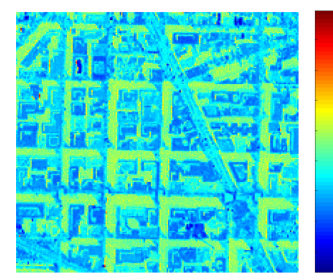

(c) $\gamma_{3}$

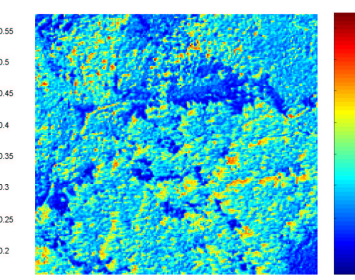

(g) $\gamma_{3}$

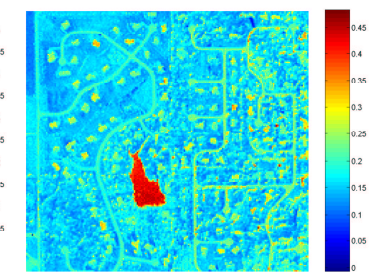

(k) $\gamma_{3}$

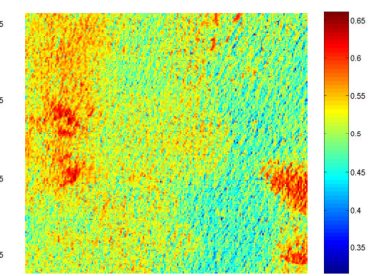

(o) $\gamma_{3}$

(p) $\gamma_{4}$

(l) $\gamma_{4}$

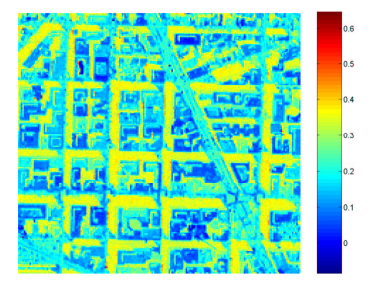

(d) $\gamma_{4}$

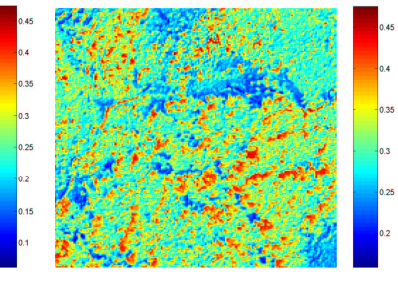

(h) $\gamma_{4}$
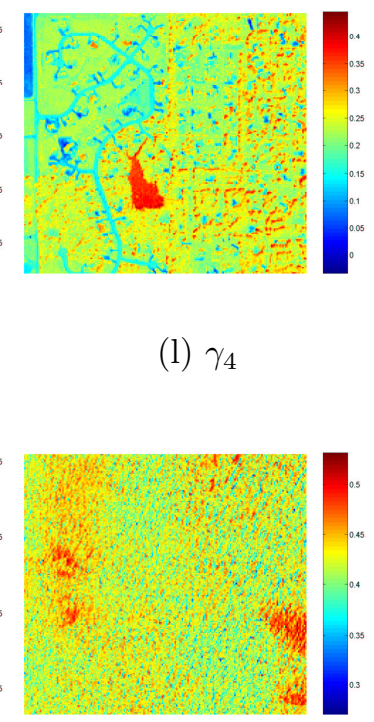

Figure 4.15: Color coded responses of the four SWI to the sample images 
main reason for $\gamma_{1}$ 's better performance on the Indiana image is that it responds low to vegetated regions in this image. Finally, for the Maryland image, both $\gamma_{1}$ and $\gamma_{2}$ perform well. Based on these observations $\gamma_{2}$ seems more robust in detecting shadows and water sources.

To compare the dynamic range of these four SWI, we next obtain the normalized histograms on the four images. In Fig. 4.13 we give these histograms; in which the solid curve represents $\gamma_{1}$, the dotted curve represents $\gamma_{2}$, the dashed and dotted curve represents $\gamma_{3}$, and the dashed curve represents $\gamma_{4}$.

If we compare these histograms, we see that $\gamma_{1}$ has the highest dynamic range especially on the Oregon and Indiana images. This is again because of its low response to vegetated regions. All other indices have similar dynamic ranges.

Next, let's focus on the two extremes (New Mexico and Florida) to compare responses of the four SWI by their sample means, $\mu$. As we recall, the New Mexico image has neither shadow nor water regions, so the best index response to this image should be a value around zero. The Florida image is of the ocean, so the best index should have the highest response to it. We give these comparisons in Table 4.4.

\begin{tabular}{|l|c|c|c|c|}
\hline & $\mu_{\gamma_{1}}$ & $\mu_{\gamma_{2}}$ & $\mu_{\gamma_{3}}$ & $\mu_{\gamma_{4}}$ \\
\hline The New Mexico image & -0.1896 & 0.1051 & 0.1649 & 0.0904 \\
\hline The Florida image & 0.6939 & 0.5147 & 0.5021 & 0.3895 \\
\hline
\end{tabular}

Table 4.4: Comparison of the four SWI on the New Mexico, and Florida images

As seen from the first row of Table 4.4, all indices except the $\gamma_{3}$ performed fairly well on responding the New Mexico image. The $\gamma_{1}$ index has a negative response to this image. This is again because of its low response to vegetation. For the Florida 


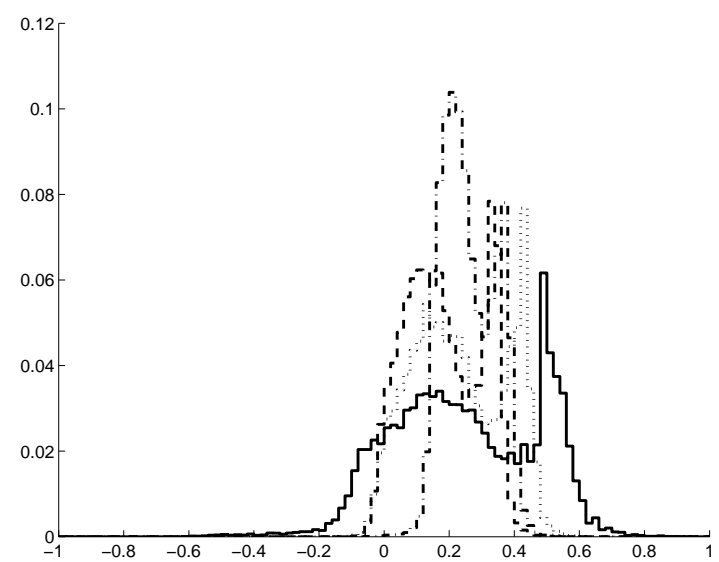

(a) Histograms for the Maryland image

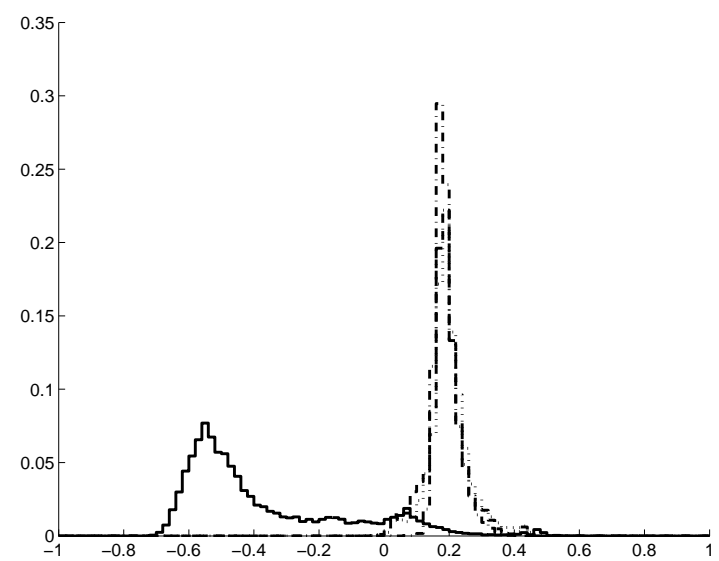

(c) Histograms for the Indiana image

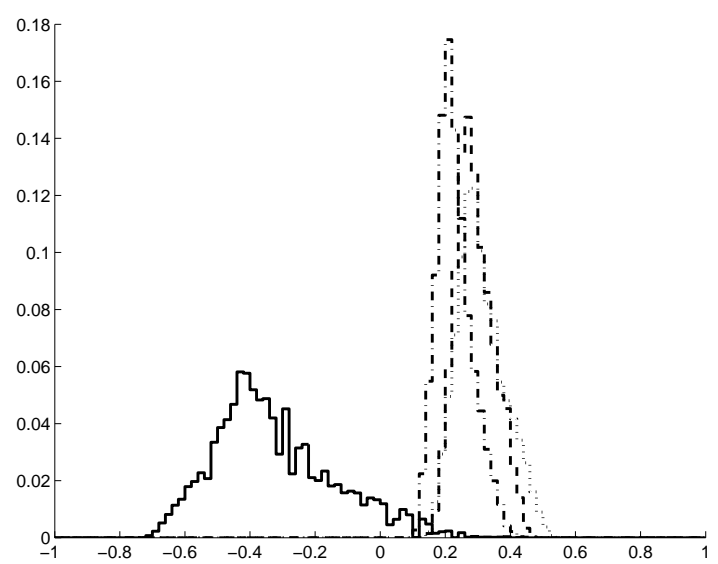

(b) Histograms for the Oregon image

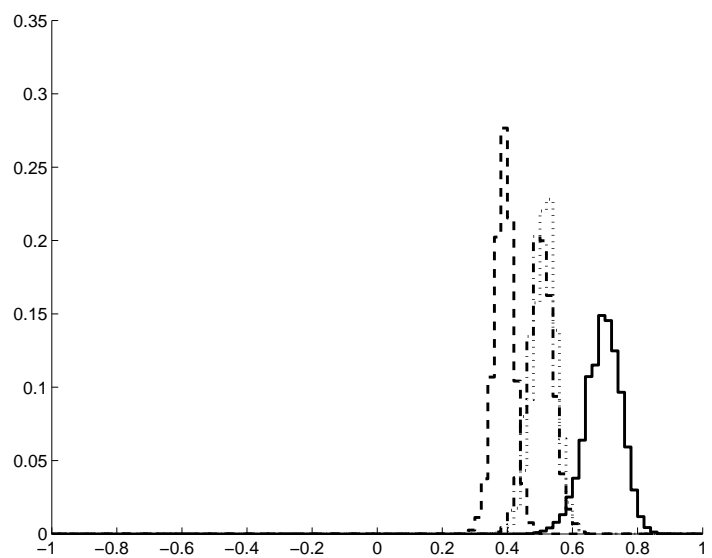

(d) Histograms for the Florida image

Figure 4.16: Comparison of histograms for the four SWI to compare their dynamic range. ( solid $-\gamma_{1}$, dot $-\gamma_{2}$, dash and dot $-\gamma_{3}$, dash $-\gamma_{4}$ ) 
image (given in the second row of Table 4.4) $\gamma_{1}$ outperformed all other indices by having the highest value. $\gamma_{2}$ and $\gamma_{2}$ have the second and third highest values. $\gamma_{4}$ performed poorly. Based on this criterion, we can sort these indices as $\gamma_{1}, \gamma_{2}, \gamma_{3}$, and $\gamma_{4}$ from best to worst. However, $\gamma_{1}$ has a tendency to respond weakly to vegetated regions, even if they contain shadows.

\subsubsection{Comparison by the Second Criterion (Entropy on High Contrast Images)}

Next, let's compare these four SWI based on their entropy on the three images containing shadow or water regions. We label images as $i m g_{i}$ for $i=1,2,3$ in the order of Maryland, Oregon, and Indiana. We give the results in Table 4.5.

\begin{tabular}{|l|c|c|c|c|}
\hline & $i m g_{1}$ & $i m g_{2}$ & $i m g_{3}$ & Mean Value \\
\hline$E_{\gamma_{1}}$ & 5.3427 & 5.1407 & 5.1733 & 5.2189 \\
\hline$E_{\gamma_{2}}$ & 4.6923 & 3.8475 & 3.5557 & 4.0318 \\
\hline$E_{\gamma_{3}}$ & 3.9121 & 3.4243 & 2.8545 & 3.3970 \\
\hline$E_{\gamma_{4}}$ & 4.3985 & 3.5483 & 3.4500 & 3.7989 \\
\hline
\end{tabular}

Table 4.5: Comparison of the entropies on three images for the four SWI

This table indicates that $\gamma_{1}$ has the highest average entropy on the three images considered. Again this is because of its low response to vegetated regions in these images. $\gamma_{2}$ has the second highest entropy. $\gamma_{4}$ and $\gamma_{3}$ have the third and fourth highest entropies on these images. Based on this criterion, we can sort these indices as $\gamma_{2}, \gamma_{4}$, and $\gamma_{3}$ from best to worst excluding $\gamma_{1}$ (since its high entropy is in fact because of low response to vegetation). 
Finally, we conclude that $\gamma_{2}$ is the most suitable index to measure shadow and water densities in multispectral images. Although $\gamma_{1}$ outperformed $\gamma_{2}$ on some experiments, it has a problem in detecting shadows in vegetated regions. Moreover, its superior numerical performance is artificially high due to that low response in vegetated regions. We next give $\gamma_{2}$ in simplified form:

$$
\gamma_{2}=\frac{4}{\pi} \arctan \frac{0.6864 \rho_{\text {blue }}+0.7253 \rho_{\text {red }}+0.0537 \rho_{\text {nir }}}{\sqrt{\rho_{\text {blue }}^{2}+\rho_{\text {red }}^{2}+\rho_{\text {nir }}^{2}}}
$$

\subsection{Summary of the Chapter}

In this chapter, we considered vegetation and shadow-water indices for subsequent processing. We developed a rigorous statistical justification for the NDVI by using principal components analysis. We then proposed a solution to the saturation problem of the NDVI. Next, we considered the relationship between the NDVI and the $R V I$ and showed that these two indices in fact capture the same information. With the statistical framework at hand, we considered more bands and introduced new vegetation and shadow-water indices. We will use these to improve land use classification next. 


\section{CHAPTER 5}

\section{Land Use Classification via Multispectral Information}

In chapter 3, our approach was based on the organization of straight lines (structure) in grayscale images. It is well-known that multispectral information also offers a great deal of information for land use classification. In this chapter, we focus on the synergistic combination of structural and multispectral information. We first extract structural features, then we extract the well-known multispectral features. Finally, we combine structural and multispectral features by using our structures as the cue regions in the multispectral images and obtain our hybrid features.

Multispectral information has been used extensively for land use classification [133-142]. To summarize the multispectral information, we use one of the well-known vegetation indices, the $N D V I$ and its linearized version $\theta$ (introduced in chapter 4). Our reasoning in using these is that most wilderness regions have high vegetation density. As we move from wilderness to the city center, we encounter less and less vegetation. The NDVI and $\theta$ therefore offers evidence for the level of development.

We applied the Bayes, Parzen window, and the nearest neighbor (NN) classifiers to label each image region. We defined a two-class problem to discriminate "urban" and "not urban" regions. Based on the classification results, the synergistic combination of structural and multispectral information outperformed both of these methods alone. 
Probabilistic relaxation further improved classification performance by introducing neighborhood constraints; we used a maximum a posteriori (MAP) decision for final class assignment.

\subsection{Statistical Feature Extraction}

Before defining our statistical measures, let's give the definitions we will use repeatedly. Besides the ones defined in chapter 3 , we have the sample variance $\sigma^{2}$; the sample skewness $\gamma_{1}$, and the sample kurtosis $\gamma_{2}$; we assume these to be selfexplanatory.

\subsubsection{Structural Features}

As we mentioned, we introduced these features in chapter 3. Based on those results, we extract four features based on the statistical properties of the lines in grayscale images. The structural features we extract are:

- Mean line length, $\mu_{l}$

- Entropy of line length, $E_{l}$

- Mean line contrast, $\mu_{c}$

- Entropy of line contrast, $E_{c}$

\subsubsection{Multispectral Features}

As multispectral features, we introduce the well-known statistics obtained from the overall vegetation index representation of the image. These are the mean $\mu$, variance $\sigma^{2}$, skewness $\gamma_{1}$, and the kurtosis $\gamma_{2}$ of the index representation. We compute these 
values both for the NDVI and $\theta$ (linearized version of the $N D V I$ ) separately. The multispectral features we extract are:

- Mean of the vegetation index; $\mu_{n d v i}$ and $\mu_{\theta}$

- Variance of the vegetation index; $\sigma_{n d v i}^{2}$ and $\sigma_{\theta}^{2}$

- Skewness of the vegetation index; $\gamma_{1, n d v i}$ and $\gamma_{1, \theta}$

- Kurtosis of the vegetation index; $\gamma_{2, n d v i}$ and $\gamma_{2, \theta}$

\subsubsection{Hybrid Features}

The following two hybrid features are the synergistic combinations of the structural and the multispectral information. As the first hybrid feature, we obtain the conditional mean of the vegetation index $(N D V I$ or $\theta)$ given that the index pixels are on a line support region $H \mu_{n d v i}, H \mu_{\theta}$. Our reasoning for this feature is that line support regions are strong indicators of the transitions in an image. If these transitions occur in urban regions, we expect less vegetation in this transition region. In wilderness (and rural) regions, we expect more. This can provide a constraint on our mean value to separate rural and the urban regions more robustly.

Similar to the reasoning above, we can calculate the conditional entropy of the vegetation index if we take our contrast value as the corresponding index value over the line support region $H E_{n d v i}, H E_{\theta}$. The hybrid features we extract are:

- Conditional mean of the vegetation index; $H \mu_{n d v i}$ and $H \mu_{\theta}$

- Conditional entropy of the vegetation index; $H E_{n d v i}$ and $H E_{\theta}$ 


\subsection{Exploiting Spatial Coherence: Probabilistic Relaxation}

In classical classifiers we treat each window as a distinct entity without regard to its neighbors. Yet, levels of development in neighboring areas are not statistically independent. Cities tend to grow from a core outwards; apart from the intervention of natural barriers such as water and mountains, land development expands more or less coherently. Based on similar reasoning, Kettig and Landgrebe [26] (among others) worked on adding spatial information to land cover classification. To exploit this observation, we applied the probabilistic relaxation to our dataset.

Probabilistic relaxation is a method that allows adjoining regions to influence one another's label probabilities by means of compatibility constraints. These compatibility constraints encode the relative (in)consistency of adjoining label-pairs. For example, regions of a common type are more likely to be neighbors than regions of different types, especially, say rural-urban. A detailed discussion of probabilistic relaxation labelling is beyond the scope of this chapter; see [30].

To initialize the relaxation, we use the normalized class membership values for the Bayes and Parzen window classifiers. For the Parzen window classifier, the likelihood value of a sample to each class is the prior distribution value of it in that class. The Bayes classifier likelihood value of a sample to each class distribution is taken as its class membership to that class. Likelihood functions for urban $M_{u}$ and rural (wilderness) $M_{w}$ classes are:

$$
M_{u}=\exp \left[\left(\mathbf{x}-\mu_{\mathbf{u}}\right) \boldsymbol{\Sigma}_{u}^{-1}\left(\mathbf{x}-\mu_{\mathbf{u}}\right)^{T}-\ln \left|\boldsymbol{\Sigma}_{u}\right|\right]
$$




$$
M_{w}=\exp \left[\left(\mathbf{x}-\mu_{\mathbf{w}}\right) \boldsymbol{\Sigma}_{w}^{-1}\left(\mathbf{x}-\mu_{\mathbf{w}}\right)^{T}-\ln \left|\boldsymbol{\Sigma}_{w}\right|\right]
$$

where $\mu$ is the sample mean and $\boldsymbol{\Sigma}$ the covariance matrix for that class. We obtain the normalized class membership value for each sample by dividing its class membership by the sum of its membership values.

In the probabilistic relaxation method, the labelling of an object is updated based on the conditional probabilities of occurrences of neighboring pairs. Probabilities of labels of all objects are updated iteratively until a globally consistent set of labels is obtained or until the label probabilities stabilize. The effect of neighboring objects is encoded through compatibility coefficients, $r_{d}\left(\lambda, \lambda^{\prime}\right)$ where $\lambda$ is a candidate label on the center region and $\lambda^{\prime}$ is a candidate label on the adjacent region in direction d. $r_{d}\left(\lambda, \lambda^{\prime}\right)$ is positive for label pairs that are likely (mutually supportive) and negative for those that are mutually inhibitory. We used Yamamoto's method [103] to construct the compatibility coefficients:

$$
r_{d}\left(\lambda, \lambda^{\prime}\right)= \begin{cases}\frac{1}{1-p(\lambda)}\left(1-\frac{p(\lambda)}{p_{d}\left(\lambda \mid \lambda^{\prime}\right)}\right) & \text { if } p(\lambda) p\left(\lambda^{\prime}\right)<p\left(\lambda, \lambda^{\prime}\right) \\ \frac{p_{d}\left(\lambda \mid \lambda^{\prime}\right)}{p(\lambda)}-1 & \text { otherwise }\end{cases}
$$

Label probabilities are updated in a parallel-iterative fashion as:

$$
\begin{aligned}
& p_{i}^{(k+1)}(\lambda)=\frac{p_{i}(\lambda)\left[1+q_{i}^{(k)}(\lambda)\right]}{\sum_{\lambda^{\prime}}\left\{p_{i}^{(k)}\left(\lambda^{\prime}\right)\left[1+q_{i}^{(k)}\left(\lambda^{\prime}\right)\right]\right\}} \\
& q_{i}^{k}(\lambda)=\frac{1}{4} \sum_{d \in(N, S, E, W)} \sum_{\lambda^{\prime}} r_{d}\left(\lambda, \lambda^{\prime}\right) p_{d}^{(k)}\left(\lambda^{\prime}\right)
\end{aligned}
$$

where $p_{d}^{(k)}\left(\lambda^{\prime}\right)$ is the $k^{\text {th }}$ estimate of the probability of label $\lambda^{\prime}$ occurring at the adjacent region in the direction $d$. The conditional label-pair probabilities $P_{d}\left(\lambda \mid \lambda^{\prime}\right)$ were 
estimated manually from the same training data used earlier. The effect of Eqn. 5.5 is to compute an adjustment to the probability of label $\lambda$ on region $i$ according to the compatibilities and probabilities of label probabilities on neighboring regions. Eqn. 5.4 renormalizes the label probabilities after all adjustments have been applied. Since any rural region can be in any neighborhood direction of any urban region, our compatibility coefficients $r_{d}\left(\lambda, \lambda^{\prime}\right)$ are independent of direction, $r\left(\lambda, \lambda^{\prime}\right)$.

We applied the probabilistic relaxation algorithm to the two class problem as follows. Class label probabilities are taken as normalized class membership values as obtained (initially) from the $\mu_{\theta}, \sigma_{\theta}^{2}, \gamma_{\theta}^{2}, H \mu_{\theta}, H E_{\theta}$ feature space (After exhaustive search over all feature combinations, this gave the best Bayes classifier). These label probabilities are then adjusted by the repeated action of Eqn. 5.4 and 5.5 until they stabilize. We then apply a Maximum A Posteriori (MAP) decision to the probabilistic relaxation algorithm results for the final labelling.

For demonstration, we give the probabilistic relaxation results for the Colorado image, which is composed mostly of rural (or wilderness) regions, in Fig. 5.1 at four stages of the process.

We observe that the region initially labelled as urban shrinks as the relaxation proceeds, correctly. Each region enforced its neighbors in accordance to the neighborhood constraints to adjust its label. Since we had pockets of urban labels among a large block of rural (wilderness) regions, and since such kind of layout is punished in the relaxation we could finally obtain better labelling. If we compare the initial and the final images we observe that although we still have regions labelled as urban regions as false alarms they are greatly reduced. 


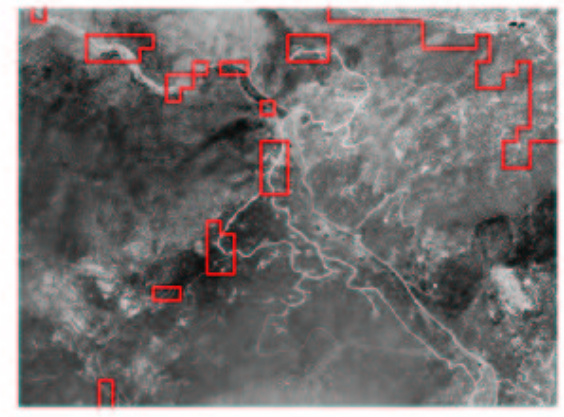

(a) iteration $=1$

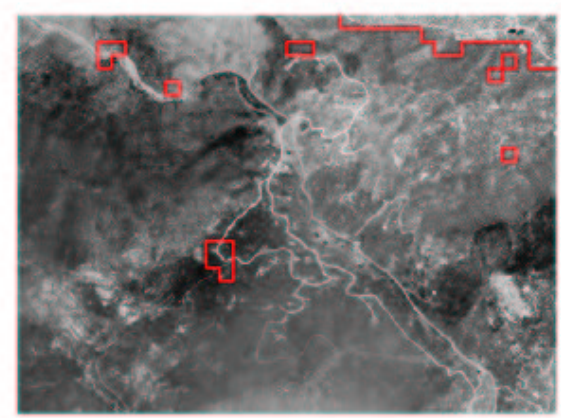

(c) iteration $=7$

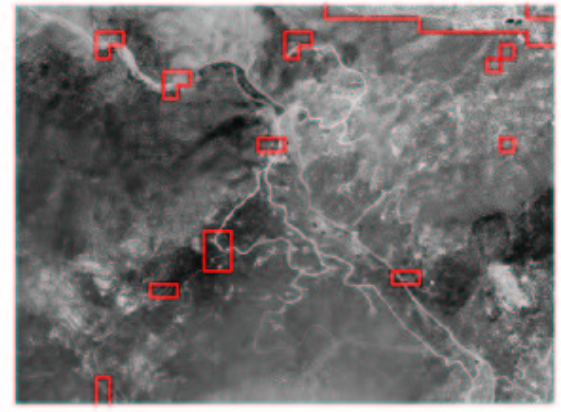

(b) iteration $=4$

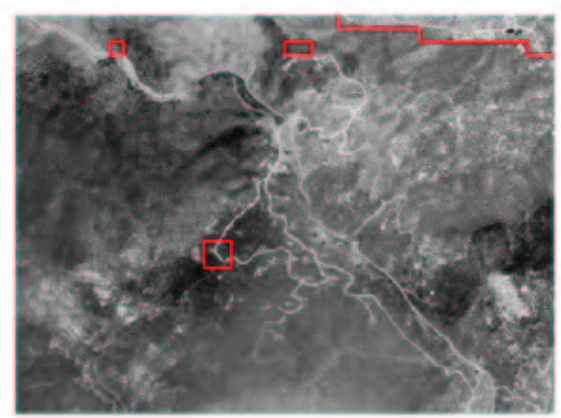

(d) iteration $=10$

Figure 5.1: The Colorado Image, probabilistic relaxation iterations

We also give two more examples for probabilistic relaxation as the Rome image and the Ecuador image. For the Rome image given in Fig. 5.2, the region initially labelled as rural expands as the relaxation proceeds, as desired. Similarly for the Ecuador image given in Fig. 5.3, the region initially labelled as rural shrinks and the region initially labelled as urban expands as the relaxation proceeds, as desired. 


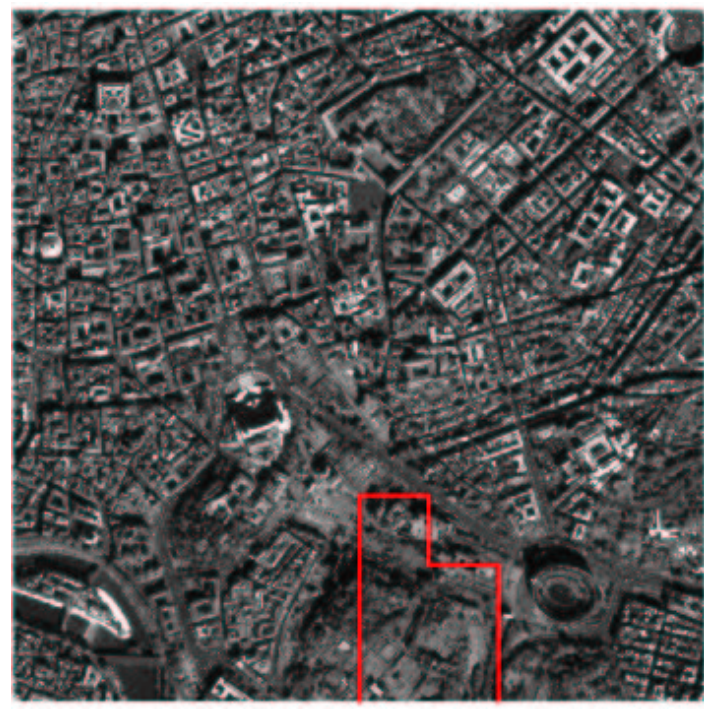

(a) Before probabilistic relaxation

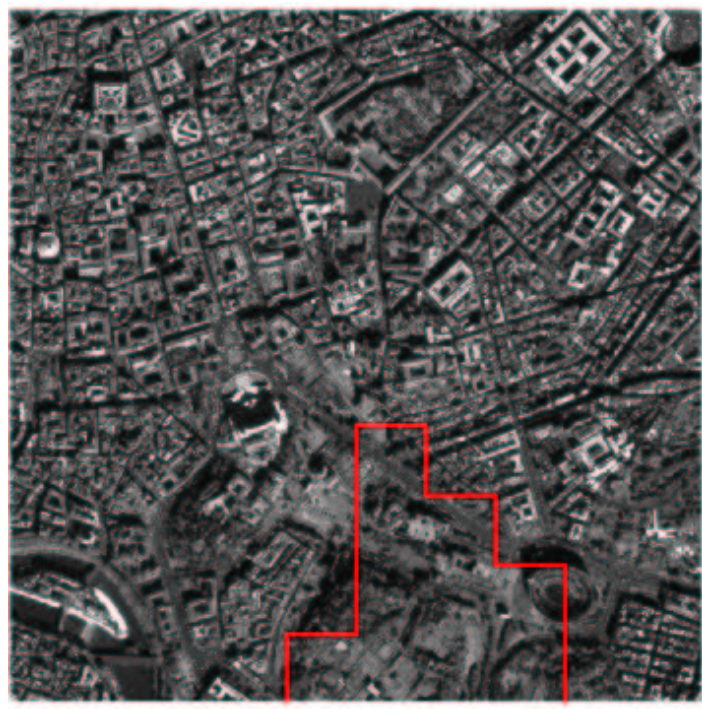

(b) After probabilistic relaxation

Figure 5.2: The Rome Image, probabilistic relaxation results

\subsection{Experimental Classification Results}

We test our features with the standard classifiers (Bayes, Parzen window, and nearest neighbor $(\mathrm{NN})$ ) and relaxation based MAP decision. We tabulated classification performances of structural, multispectral, and hybrid features separately so that we can compare them. For each set of features (structural, multispectral, and hybrid), we applied an exhaustive search over the full feature set (all singles, pairs, triples, etc.) to obtain the best performing ones. To observe the affect of linearization, we also tabulated the classification performances of the NDVI and $\theta$ based features separately.

Our data set is composed of one meter resolution panchromatic and the corresponding four meter resolution multispectral images of North American regions. We 


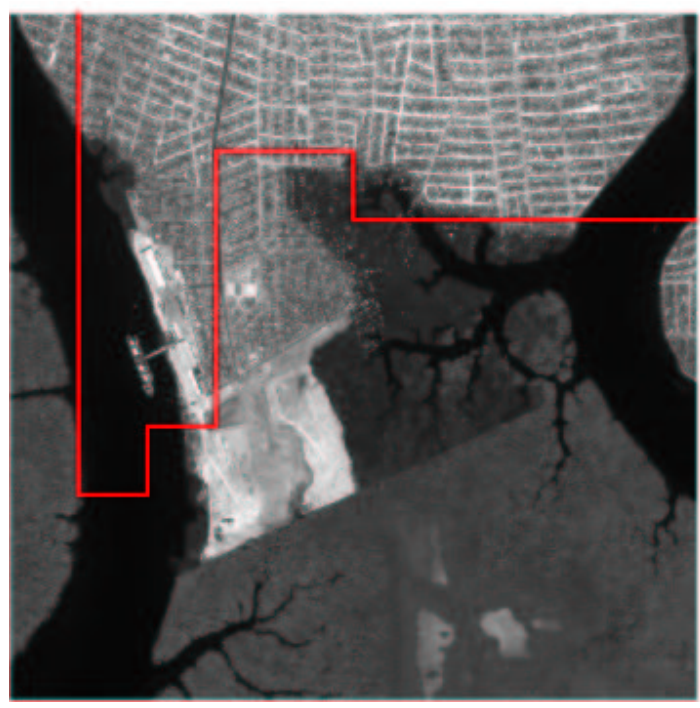

(a) Before probabilistic relaxation

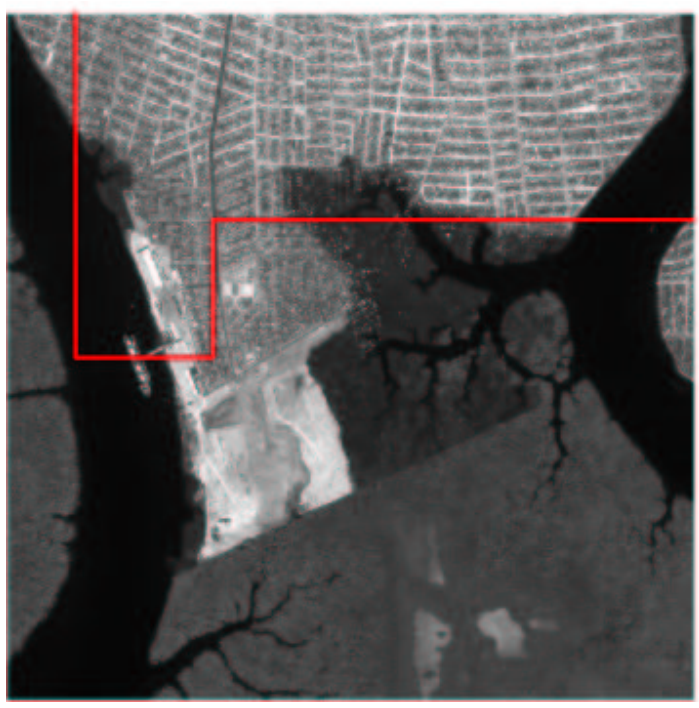

(b) After probabilistic relaxation

Figure 5.3: The Ecuador Image, probabilistic relaxation results

give all of the images forming this data set in Table 5.1. We extract statistical features over $800 \times 800$ pixel windows. We discard straight lines shorter than five pixels (5 meters); such objects can not be approximated robustly due to low signal to noise ratios in the contour approximation and certainly do not offer strong evidence for "straightness".

We demonstrate the classification performances of structural features, multispectral features (NDVI and $\theta$ separately), hybrid features (with additional multispectral features), and MAP decisions (for Bayes and Parzen window) Fig. 5.4. Exact values with the feature spaces are given in Appendix 5.4. In these figures, we provide the average classification performances and urban classification performances separately. Average classification is the correct classification rates of urban and rural test 
samples. Urban classification performance is the correct classification of urban test samples only. For our application, miss rates in urban detection are more important than false alarms. Since these false alarms may be recovered in the future steps. However, misses can not be recovered that easily.

As can be seen in these figures, we have a clear improvement in each step for urban and average classification performances. Next, we provide this information in tabular form in Table 5.2.

To compare our hybrid features with the structural or multispectral features alone, we take the multispectral feature performance as a benchmark. To be more fair we did not take structural features, since they had slightly poor performance compared to the multispectral ones. As can be seen, the improvements for the average classification are $\mathbf{8 . 6} \%, 1.7 \%$, and $2.5 \%$ for the Bayes, Parzen window, and NN classifiers respectively.

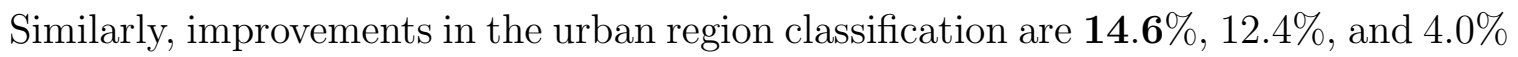
for the Bayes, Parzen window, and NN classifiers respectively.

As can be seen in Table 5.2, multispectral features perform better than the structural features. The main reason for the relatively poor performance of the structural features is the window size (Structural features are sensitive to the window size). In chapter 3 , the window size was $400 \times 400$ pixels; here it is $800 \times 800$ pixels.

To compare the NDVI and $\theta$ features, we tabulate their performances in Table 5.3. For the average classification performance, we have around $1 \%$ improvement for all three classifiers. These improvements may not seem significant, however there is almost no cost for these improvements (We just take the arctangent of the NDVI to obtain the $\theta$ representation). If we focus on urban region detection rates, we have significant improvements in the range of $1.1-4.5 \%$. These results suggest that, $\theta$ 


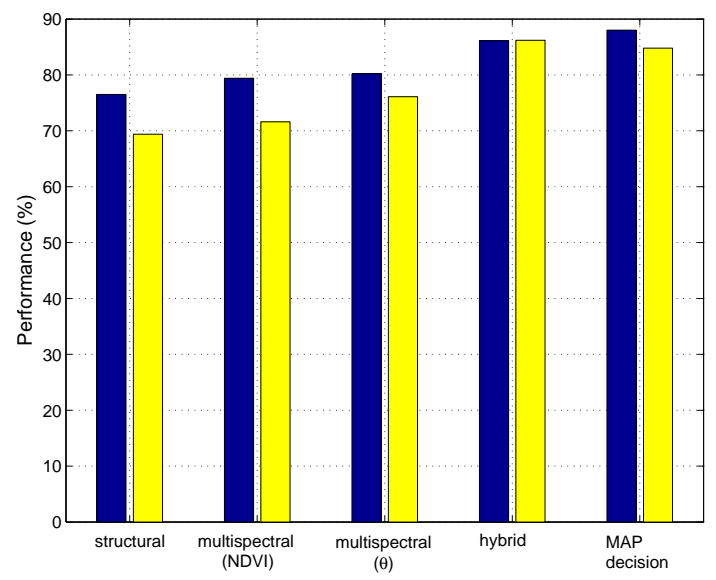

(a) Bayes classifier

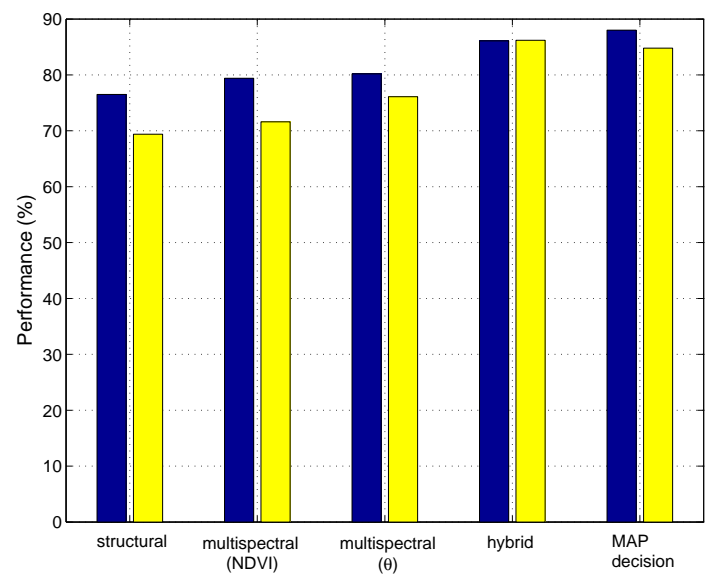

(b) Parzen window classifier

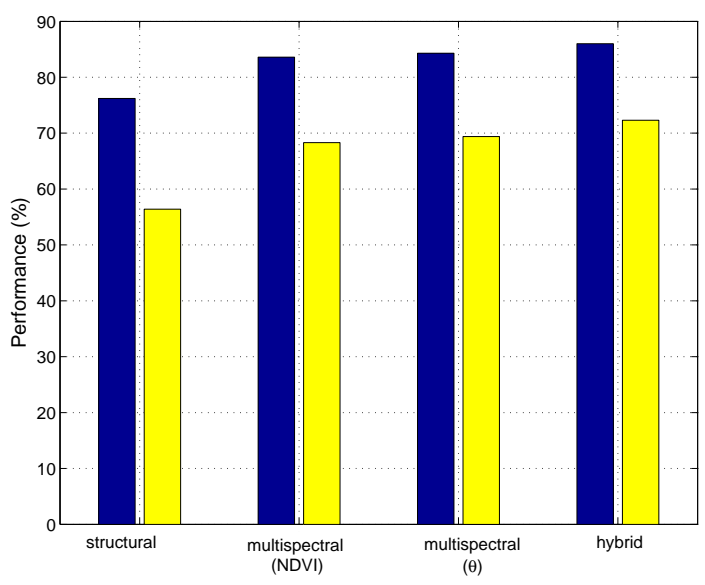

(c) NN classifier

Figure 5.4: Improvements in classification for the Bayes, Parzen window, and NN classifiers. Dark bars correspond to urban performances, light bars correspond to average performances. 
representation not only linearize the $N D V I$ as we suggested in chapter 4 , it also improves classification performances.

Finally, let's consider the affect of adding spatial information to classification (MAP decision after relaxation). If we consider the average classification, we have $\mathbf{1 . 8} \%$, and $0.2 \%$ improvements by using the MAP decision over the hybrid features for the Bayes and the Parzen window classifiers. The improvements here are marginal if we take the computation cost of relaxation into account. If we focus on urban region detection rates, we have a drop of $1.4 \%$ for the Bayes classifier and a $\mathbf{1 1 . 1 \%}$ improvement for the Parzen window classifier. Although we have a deterioration in the Bayes classifier, the improvement in the Parzen window classifier is remarkable.

If we sum up, for some cases we obtained almost a $\mathbf{9 \%}$ improvement for average classification and a $\mathbf{1 5 \%}$ improvement for urban region classification by using hybrid features, $\theta$ representation, and spatial information successively. Since we are more interested in detecting urban regions, this improvement is substantial.

\subsection{Appendix: Classification Performances}

We give the feature spaces, number of training and test samples for each classifier separately. Each classifier has benefits and shortcomings. Bayes classifier assumes a parametric model for the data distribution. This model is in the form of a Gaussian distribution for most of the times. If the data represents such a characteristic, the classifier performs well. One strength of the Bayes classifier is its low computational cost. On the other hand, Parzen window and NN classifiers does not assume a priori model for the data distribution. Instead, they model the data distribution locally. The main drawback of these two methods is their computational cost. 
We start explaining the design criteria of the Bayes classifier. To train this classifier, we used 112 rural and 136 urban image windows selected at random from the overall data set. We then tested 5657 new region samples (3903 rural, 1754 urban) to ascertain the classification performances. We give the best performances in Table 5.4. We have discussed it in detail in Section 5.3.

Next, we consider the Parzen window classifier. To train this classifier, we used 419 rural and 419 urban image windows selected at random from the overall data set. We then tested 5160 new region samples (3449 rural, 1611 urban) to ascertain the classification performances. We give the best performances in Table 5.5. We have discussed it in detail in Section 5.3.

Finally, we consider the NN classifier. To train this classifier, we used 419 rural and 419 urban image windows selected at random from the overall data set. We then tested 5160 new region samples (3411 rural, 1649 urban) to ascertain the classification performances. We give the best performances in Table 5.6. We have discussed it in detail in Section 5.3.

\subsection{Summary of the Chapter}

In this chapter, we improved our previous results in assessing the degree of land development in three steps. Our first step was to include $\theta$ as the multispectral information. Our second step was to synergistical combination of the structural and the multispectral information. Our last step was using the spatial information in classification. Next, we introduce more selective but computationally more expensive graph theoretical measures to extract residential regions. 


\begin{tabular}{|l|c|c|}
\hline GEOGRAPHIC LOCATION & ACQUISITION DATE & IMAGE SIZE $\left(\mathrm{m}^{2}\right)$ \\
\hline Arizona & $7 / 23 / 005: 55 \mathrm{PM}$ & $6508 \times 6500$ \\
\hline Arizona & $8 / 14 / 005: 57 \mathrm{PM}$ & $5588 \times 4696$ \\
\hline California & $4 / 5 / 006: 16 \mathrm{PM}$ & $11372 \times 8992$ \\
\hline California & $3 / 15 / 006: 48 \mathrm{PM}$ & $22092 \times 13312$ \\
\hline California & $3 / 18 / 006: 59 \mathrm{PM}$ & $19608 \times 15488$ \\
\hline California & $3 / 18 / 006: 59 \mathrm{PM}$ & $11264 \times 15544$ \\
\hline California & $3 / 18 / 006: 59 \mathrm{PM}$ & $10868 \times 15580$ \\
\hline California & $3 / 18 / 006: 59 \mathrm{PM}$ & $23344 \times 13808$ \\
\hline California & $3 / 18 / 006: 59 \mathrm{PM}$ & $12544 \times 13860$ \\
\hline California & $3 / 18 / 006: 59 \mathrm{PM}$ & $12544 \times 13860$ \\
\hline California & $3 / 26 / 006: 51 \mathrm{PM}$ & $13824 \times 12972$ \\
\hline California & $3 / 26 / 006: 51 \mathrm{PM}$ & $13596 \times 12972$ \\
\hline California & $8 / 18 / 006: 40 \mathrm{PM}$ & $16772 \times 12456$ \\
\hline Florida & $5 / 20 / 003: 44 \mathrm{PM}$ & $7424 \times 6760$ \\
\hline Idaho & $5 / 30 / 006: 21 \mathrm{PM}$ & $5188 \times 7176$ \\
\hline Indiana & $5 / 24 / 004: 25 \mathrm{PM}$ & $6436 \times 6448$ \\
\hline Indiana & $5 / 24 / 004: 24 \mathrm{PM}$ & $6492 \times 6284$ \\
\hline Indiana & $7 / 1 / 004: 12 \mathrm{PM}$ & $8160 \times 8140$ \\
\hline Indiana & $6 / 1 / 004: 17 \mathrm{PM}$ & $5100 \times 5092$ \\
\hline Indiana & $9 / 27 / 004: 22 \mathrm{PM}$ & $6436 \times 6448$ \\
\hline Indiana & $8 / 14 / 004: 17 \mathrm{PM}$ & $6436 \times 6448$ \\
\hline Kansas & $5 / 11 / 004: 50 \mathrm{PM}$ & $11436 \times 11196$ \\
\hline Maryland & $6 / 3 / 003: 50 \mathrm{PM}$ & $11348 \times 11132$ \\
\hline Maryland & $4 / 1 / 003: 52 \mathrm{PM}$ & $10000 \times 10000$ \\
\hline Minnesota & $2 / 16 / 004: 47 \mathrm{PM}$ & $5916 \times 8660$ \\
\hline Minnesota & $4 / 25 / 005: 03 \mathrm{PM}$ & $5916 \times 8660$ \\
\hline Mississippi & $7 / 26 / 004: 25 \mathrm{PM}$ & $5316 \times 7008$ \\
\hline Mississippi & $7 / 21 / 004: 43 \mathrm{PM}$ & $6172 \times 4008$ \\
\hline Mississippi & $8 / 3 / 004: 17 \mathrm{PM}$ & $5076 \times 5084$ \\
\hline Mississippi & $9 / 27 / 004: 23 \mathrm{PM}$ & $5076 \times 5084$ \\
\hline Mississippi & $7 / 21 / 004: 43 \mathrm{PM}$ & $5216 \times 4996$ \\
\hline Missouri & $2 / 29 / 004: 25 \mathrm{PM}$ & $7340 \times 7360$ \\
\hline Missouri & $2 / 29 / 004: 25 \mathrm{PM}$ & $7340 \times 7360$ \\
\hline New Mexico & $5 / 23 / 005: 28 \mathrm{PM}$ & $10500 \times 10776$ \\
\hline Oklahoma & $5 / 22 / 004: 52 \mathrm{PM}$ & $11324 \times 11004$ \\
\hline Oregon & $8 / 8 / 007: 14 \mathrm{PM}$ & $11128 \times 11092$ \\
\hline South Dakota & $5 / 20 / 005: 16 \mathrm{PM}$ & $11420 \times 11260$ \\
\hline Wyoming & $6 / 26 / 006: 06 \mathrm{PM}$ & $14068 \times 10188$ \\
\hline & & \\
\hline
\end{tabular}

Table 5.1: Test images 


\begin{tabular}{|c|c|c||c|c||c|c|}
\hline \multirow{2}{*}{ Classifier/Features } & \multicolumn{6}{|c|}{ Performance (\%) } \\
\cline { 2 - 7 } & \multicolumn{2}{|c|}{ Structural } & \multicolumn{2}{c|}{ Multispectral } & \multicolumn{2}{c|}{ Hybrid } \\
\hline & urban & average & urban & average & urban & average \\
\hline Bayes & 69.4 & 76.5 & 71.6 & 79.4 & 86.2 & 86.1 \\
\hline Parzen window & 54.6 & 75.3 & 70.2 & 84.7 & 72.7 & 86.2 \\
\hline NN & 56.4 & 76.2 & 68.3 & 83.6 & 72.3 & 86.0 \\
\hline
\end{tabular}

Table 5.2: Comparison of detection performances

\begin{tabular}{|c|c|c||c|c|}
\hline \multirow{2}{*}{ Classifier/Features } & \multicolumn{4}{|c|}{ Performance (\%) } \\
\cline { 2 - 5 } & \multicolumn{2}{|c|}{ NDVI } & \multicolumn{2}{c|}{$\theta$} \\
\hline & urban & average & urban & average \\
\hline Bayes & 71.6 & 79.4 & 76.1 & 80.2 \\
\hline Parzen window & 70.2 & 84.7 & 71.4 & 85.5 \\
\hline NN & 68.3 & 83.6 & 69.4 & 84.3 \\
\hline
\end{tabular}

Table 5.3: Comparison of detection performances

\begin{tabular}{|l|l|c|c|c|}
\hline \multirow{2}{*}{ Method } & & \multicolumn{3}{|c|}{ Performance (\%) } \\
\cline { 3 - 5 } & Feature space & rural & urban & average \\
\hline Structural & $\mu_{c}, E_{c}$ & 79.6 & 69.4 & 76.5 \\
\hline Multispectral $(N D V I)$ & $\mu_{n d v i}, \sigma_{n d v i}^{2}$ & 83.0 & 71.6 & 79.4 \\
\hline Multispectral $(\theta)$ & $\mu_{\theta}, \sigma_{\theta}^{2}$ & 82.1 & 76.1 & 80.2 \\
\hline Hybrid & $\mu_{\theta}, \sigma_{\theta}^{2}, \gamma_{2, \theta}, H \mu_{\theta}, H E_{\theta}$ & 86.1 & 86.2 & 86.1 \\
\hline MAP decision & $\mu_{\theta}, \sigma_{\theta}^{2}, \gamma_{2, \theta}, H \mu_{\theta}, H E_{\theta}$ & 89.4 & 84.8 & 88.0 \\
\hline
\end{tabular}

Table 5.4: Comparison of performances for the Bayes classifier

\begin{tabular}{|l|l|c|c|c|}
\hline \multirow{2}{*}{ Method } & & \multicolumn{3}{|c|}{ Performance (\%) } \\
\cline { 3 - 5 } & Feature space & rural & urban & average \\
\hline Structural & $\mu_{c}, E_{c}$ & 88.7 & 54.6 & 75.3 \\
\hline Multispectral $(N D V I)$ & $\mu_{n d v i}, \sigma_{n d v i}^{2}, \gamma_{1, n d v i}, \gamma_{2, n d v i}$ & 91.7 & 70.2 & 84.7 \\
\hline Multispectral $(\theta)$ & $\mu_{\theta}, \sigma_{\theta}^{2}, \gamma_{1, \theta}, \gamma_{2, \theta}$ & 92.2 & 71.4 & 85.5 \\
\hline Hybrid & $\mu_{\theta}, \sigma_{\theta}^{2}, \gamma_{2, \theta}, H \mu_{\theta}, H E_{\theta}$ & 92.6 & 72.7 & 86.2 \\
\hline MAP decision & $\mu_{\theta}, \sigma_{\theta}^{2}, \gamma_{2, \theta}, H \mu_{\theta}, H E_{\theta}$ & 87.5 & 83.7 & 86.4 \\
\hline
\end{tabular}

Table 5.5: Comparison of performances for the Parzen window classifier 


\begin{tabular}{|l|l|c|c|c|}
\hline & & \multicolumn{3}{|c|}{ Performance (\%) } \\
\cline { 3 - 5 } Method & Feature space & rural & urban & average \\
\hline Structural & $\mu_{c}, E_{c}$ & 86.8 & 56.4 & 76.2 \\
\hline Multispectral $(N D V I)$ & $\mu_{n d v i}, \sigma_{n d v i}^{2}, \gamma_{1, n d v i}$ & 91.0 & 68.3 & 83.6 \\
\hline Multispectral $(\theta)$ & $\mu_{\theta}, \sigma_{\theta}^{2}, \gamma_{1, \theta}$ & 91.5 & 69.4 & 84.3 \\
\hline Hybrid & $\mu_{\theta}, \sigma_{\theta}^{2}, \gamma_{2, \theta}, H \mu_{\theta}, H E_{\theta}$ & 92.6 & 72.3 & 86.0 \\
\hline
\end{tabular}

Table 5.6: Comparison of performances for the NN classifier 


\section{CHAPTER 6}

\section{Extracting Residential Regions by Graph Theoretical Measures}

In the previous chapters, we were able to eliminate wilderness regions via synergistic combination of structural and multispectral information. Therefore we are left with urban and residential regions at this step. For subsequent processing (house and street network detection) we need more selective features to discriminate residential regions of specific type. Therefore, we introduce graph theoretical measures over grayscale images here.

The graphs are constructed using lines (obtained from line support regions, see chapter 3) as vertices, while graph edges encode their spatial relationships. We then introduce a set of measures based on various properties of the graph. These measures are monotonic with increasing structure (organization) in the image. Thus, increased cultural activity and land development are indicated by increases in these measures without explicit extraction of road networks, buildings, residences etc. These latter, time consuming (and still only partially automated) tasks can be restricted only to "promising" image regions, according to our measures. In some applications our measures may suffice. We present a theoretical basis for the measures followed by extensive experimental results in which the measures are first compared to manual 
evaluations of land development. We then present and test a method to focus on, and (pre)extract, suburban-style residential areas. These are of particular importance in many applications, and particularly difficult to extract.

\subsection{Overview and Prior Work}

\subsubsection{Overview}

Our system extracts primitives from the image, calculates measures on these primitives, and fuses these measures to determine the final region type. Our primitives are lines extracted by line support regions, as given in chapter 3. We represent each straight line (segment) as a vertex in a graph and define a neighborhood tolerance to construct edges between these vertices. Based on these graphs, we introduce measures to assess the type of region. These measures increase monotonically with respect to the degree of organization in the image. To form these measures, we first consider unweighted graphs and use the circuit rank and degree (valency) sequence. Then, we consider weighted graphs and introduce measures based on graph partitioning and the graph spectrum.

For our purposes, the level of development (degree of organization) is based on the type and density of construction (buildings, streets, etc.) and its geometric regularity. Our measures are defined to infer this indirectly from the organization of lines in the image. We consider rural areas without buildings to be the least developed, proceeding through sparse residential, dense residential, commercial, industrial, to city centers.

We conducted two sets of experimental investigations to evaluate these measures. The first set is designed to verify their monotonic behavior. Because the notion of 
"ground truth" is speculative, at best, in this setting, we asked 90 people to sort a set of 20 test images according to their assessment of the degree of organization. We then compare our measures to the consensus ordering. Although each measure performed reasonably well on its own, performance improves significantly when the measures are fused, as we describe below.

Our second set of tests seeks to determine the discriminability of these measures. Therefore, we focused on sparse residential areas. These areas, often including large, overhanging trees, exhibit forest-like characteristics and present a significant challenge. Yet, they are also of particular importance because it is often in these areas that growth most rapidly occurs. To detect these regions, we implemented and tested two strategies. In the first, we developed a Neyman-Pearson decision system based on the Mahalonobis distance to the center of the residential region's distribution in our graph theoretical feature space. The second approach is to define a three class

problem (rural, residential, urban) with a Bayes classifier. We obtained very good results in both cases. It is particularly noteworthy that the residential regions show a low miss rate.

\subsubsection{Prior Work}

There exists relatively little prior work in the direct assessment of land development from overhead views based on image structure (vs. pixel by pixel radiometry). Among the more notable - and philosophically most similar - is the work of Sarkar and Boyer in change detection from graph spectra [178]. Because they were looking at smaller areas (construction sites, typically) and because their images were not metric 
(as ours are), they faced a somewhat different environment. Our graphs are simpler to construct, and our measures are (potentially) more discriminating.

We considered another set of structural features to discriminate rural and urban regions specifically in chapter 3 . Those features provided reasonable performance while being simpler to compute. We also benefit from them in this chapter. The new measures described here are more discriminating.

Other work in land development (or use) classification is overwhelmingly a matter of pixel (or small neighborhood) classification based on radiometric properties. The literature is vast, but for some examples see [21].

\subsection{Graph Construction and Consensus Ordering}

In abstracting the image to a graph, we make the following observations. First, the impact of human activity, especially development, is manifested in the emergence of straight and smoothly curved contours. Second, the spatial density and regularity of these contours increases with increasing development. Here, we concentrate on straight lines extracted by line support regions (chapter 3). These line segments correspond to the vertices of the graph.

Vertices may be attributed by the length, orientation, contrast given in Eqn. 3.15, and centroid location of the corresponding straight line segment. Edges connect vertices corresponding to lines that are sufficiently close to one another. For the purpose of edge construction, the distance between two lines is the minimum distance between their point sets. We tested neighborhood tolerances of 3, 5, and 10 meters (pixels); experimentally we find that 5 works well. An edge may be weighted according to a function of the attributes of the vertices it joins. 
Each image in our data set is $800 \times 800$ pixels; we have hundreds of test images at hand. Of these, we selected 20 "representatives" covering the range of development from wilderness/open water to dense city-center. These representative images appear in Fig. 6.1, and their extracted lines in Fig. 6.2. The geographical location and the type of each sample test image is summarized in Table 6.1.

\begin{tabular}{|c|l|l|}
\hline Image \# & Image Name & Image Type \\
\hline 1 & Idaho & Rural \\
\hline 2 & Minnesota & Forest \\
\hline 3 & Kansas & Rural \\
\hline 4 & Minnesota & Rural \\
\hline 5 & Oregon & Forest \\
\hline 6 & Florida & Sea \\
\hline 7 & Oklahoma & Farmland \\
\hline 8 & Oklahoma & Farmland \\
\hline 9 & South Dakota & Sparse residential \\
\hline 10 & Maryland & Sparse residential \\
\hline 11 & Indiana & Sparse residential \\
\hline 12 & New Mexico & Sparse residential \\
\hline 13 & California & Dense residential \\
\hline 14 & California & Commercial \\
\hline 15 & Missouri & Dense residential \\
\hline 16 & California & Industrial \\
\hline 17 & California & Dense residential \\
\hline 18 & Maryland & City center \\
\hline 19 & Arizona & Dense residential \\
\hline 20 & California & City center \\
\hline
\end{tabular}

Table 6.1: Geographical locations and types of the sample test images

We asked 90 people to sort the images from least organized ( $($ score $=20)$. We defined the consensus ordering according to the median scores. For regions with discernible development activity, the responses were fairly consistent. 


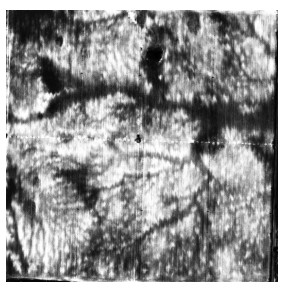

(a) Image 1

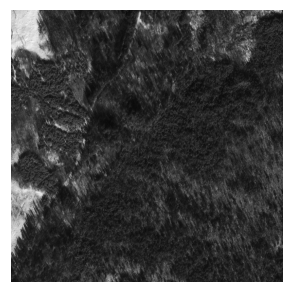

(b) Image 2

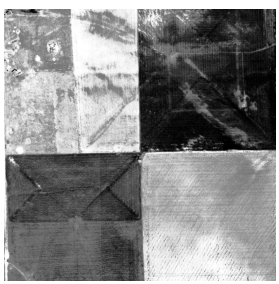

(g) Image 7

(f) Image 6

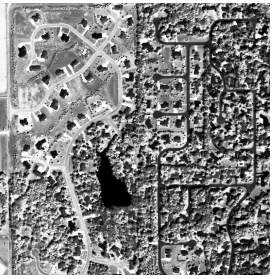

(k) Image 11

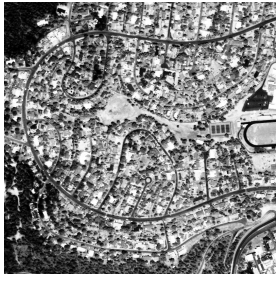

(l) Image 12

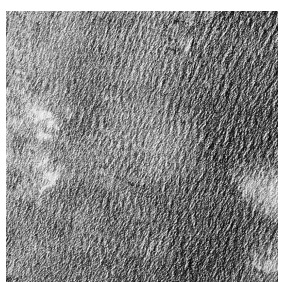

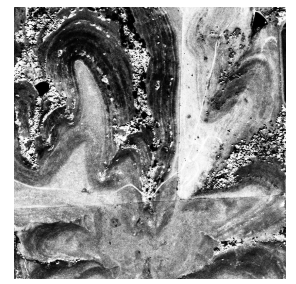

(c) Image 3

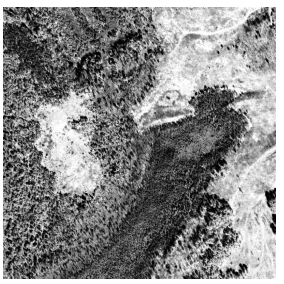

(d) Image 4

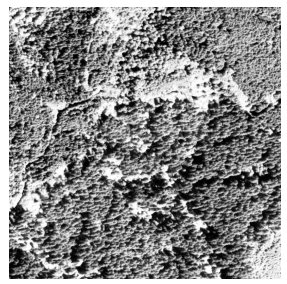

(e) Image 5

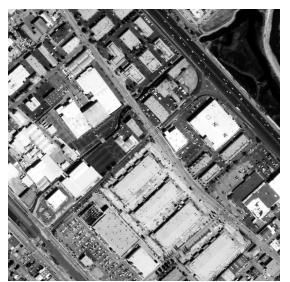

(p) Image 16

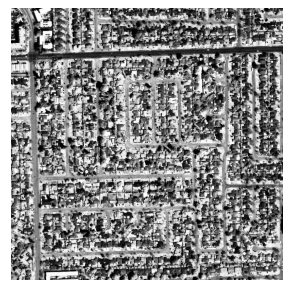

(q) Image 17

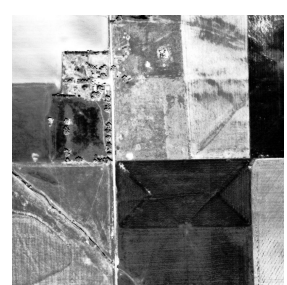

(h) Image 8

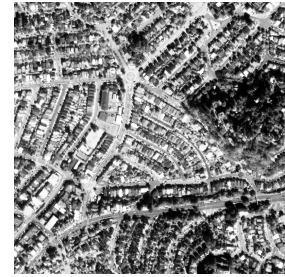

(m) Image 13

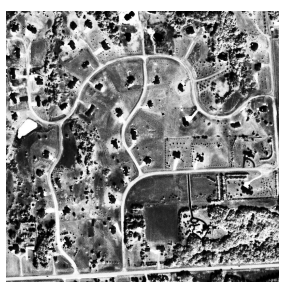

(i) Image 9

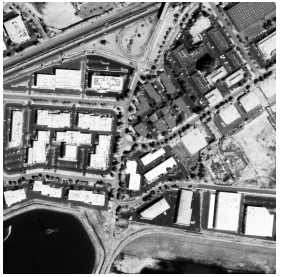

(n) Image 14

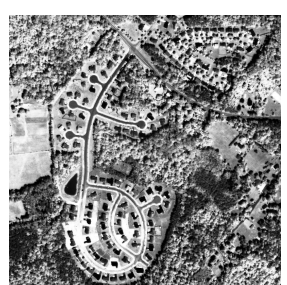

(j) Image10

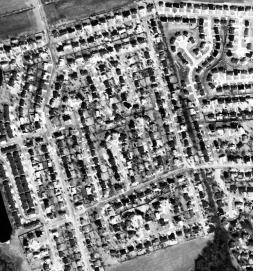

(o) Image 15

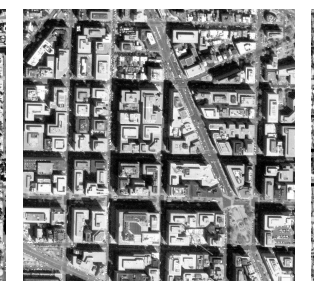

(r) Image 18

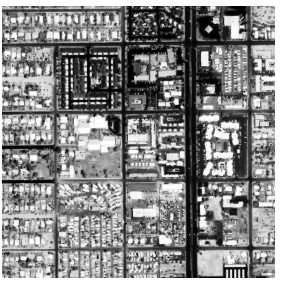

(s) Image 19

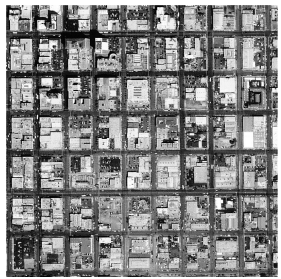

(t) Image 20

Figure 6.1: Sample test images arranged in increasing order by consensus ordering 


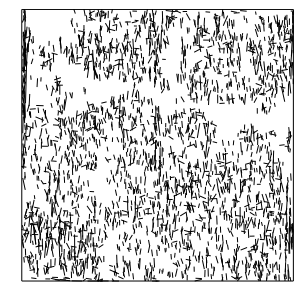

(a) Image 1

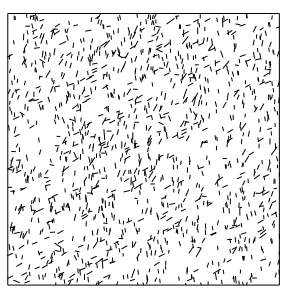

(f) Image 6

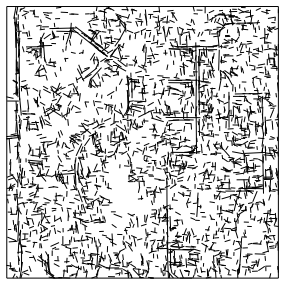

(k) Image 11

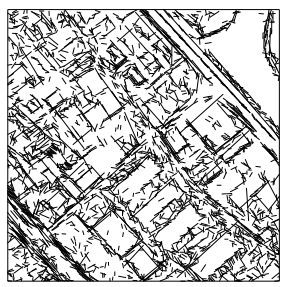

(p) Image 16

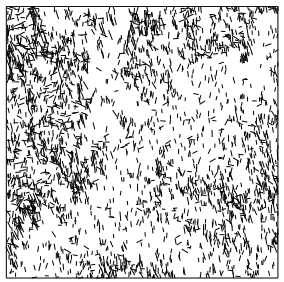

(b) Image 2

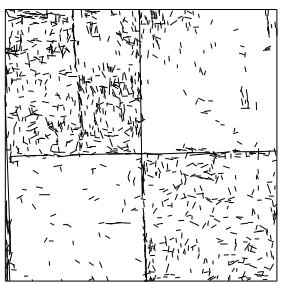

(g) Image 7

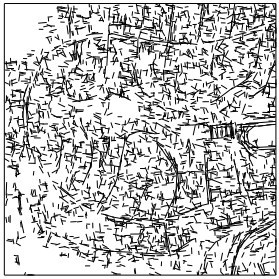

(l) Image 12

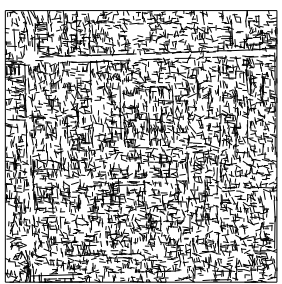

(q) Image 17

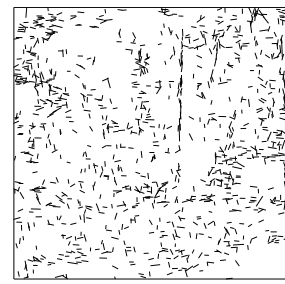

(c) Image 3

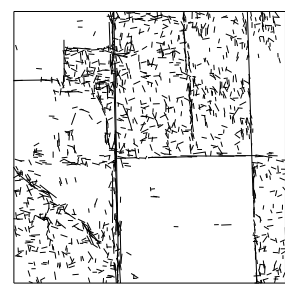

(h) Image 8

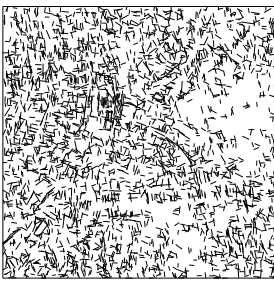

(m) Image 13

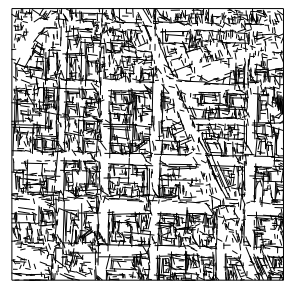

(r) Image 18

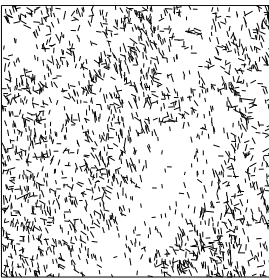

(d) Image 4

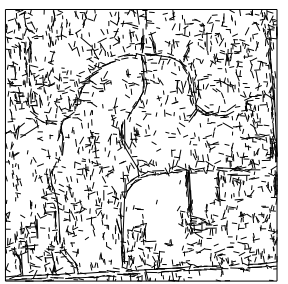

(i) Image 9

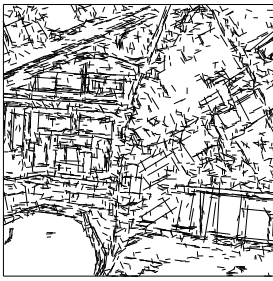

(n) Image 14

(o) Image 15

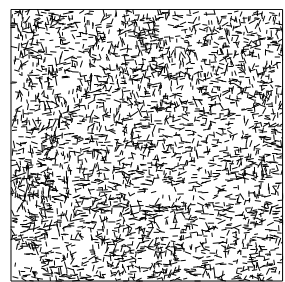

(e) Image 5 (j) Image10
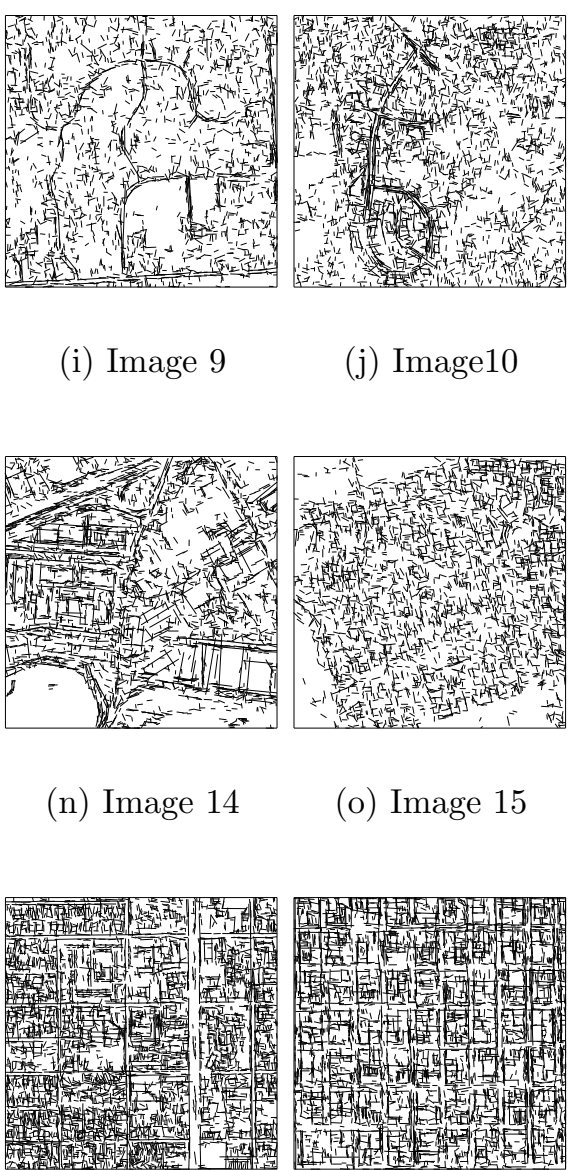

(s) Image 19

(t) Image 20

Figure 6.2: Lines obtained from the sample test images 
For regions with little to no development, the human subjects were less in agreement - but they were consistent in ranking these images below those showing development.

For the sample images, we obtain the score distributions (represented with the upper and lower quartiles and the medians) as in Fig. 6.3. As can be seen, the median scores are sufficiently close to what we expect. The first six images received fewer scores than the remaining ones and there is a clear distinction in organization scores between the sixth and seventh images.

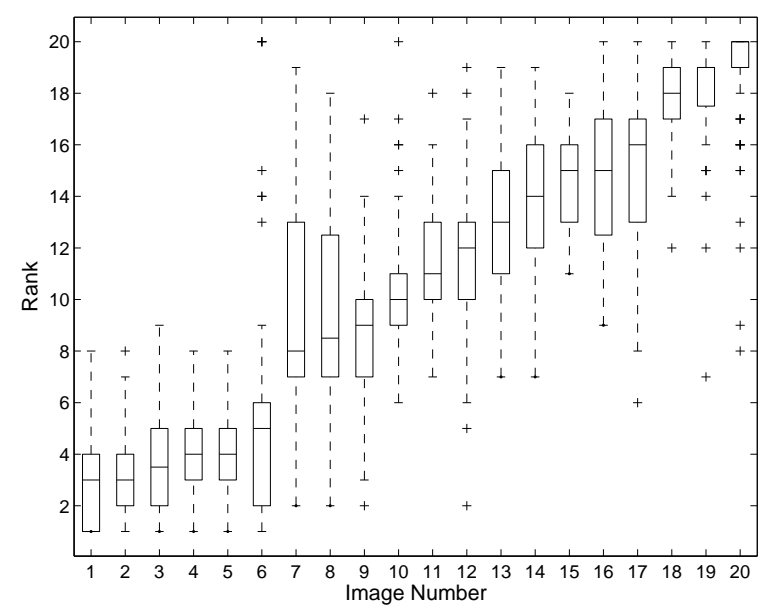

Figure 6.3: Consensus ordering and the score distribution

\subsection{Measures Based on Unweighted Graphs}

For our first investigation, we consider unweighted graphs. Our first measure is based on the circuit rank. For our second measure, based on the degree sequence, we apply a random graph formulation. These measures capture the underlying structure of the graph. 


\subsubsection{Circuit Rank}

Lines produced by the same house or building are likely to produce a cycle in the graph. A cycle is a close path of alternating vertices and edges. Thus, cycles will be prevalent in graphs constructed from dense urban regions (or in the corresponding subgraphs), but rare in graphs (subgraphs) constructed from unpopulated areas. Based on these constraints, we expect the circuit rank to increase monotonically as we move from rural regions to the city center.

Berge [179] defines the circuit rank (cyclomatic number) of graph $G$ as

$$
R(G)=m-n+c
$$

where $n$ is the number of vertices, $m$ is the number of edges, and $c$ is the number of connected components. He also proved that the circuit rank is equal to the maximum number of independent cycles. Therefore, we take the circuit rank of a graph as our first measure $m_{c r}=R(G)$.

\subsubsection{The Degree Sequence}

Let $G$ be a graph with vertex set $V=\left\{v_{1}, v_{2}, \ldots, v_{n}\right\}$. The number of edges incident on a given vertex $v_{i}$ is called the degree of $v_{i}$ and is denoted by $\operatorname{deg}\left(v_{i}\right)$. The list of numbers $\left(\operatorname{deg}\left(v_{1}\right), \operatorname{deg}\left(v_{2}\right), \ldots, \operatorname{deg}\left(v_{n}\right)\right)$ is called a degree sequence of $G$. Bollobas [180] studied random graphs to obtain the distribution of their degree sequences. Following Bollobas, we use the random graph model $\mathbf{G}(n, P(e d g e)=p)$. The model consists of all graphs with vertex set $V$ for which edges are chosen independently and with probability $p$. Our motivation for this measure is that as the region becomes more 
organized, the edge formation probability $p$ increases along with the number of vertices $n$.

Bollobas proved that the degree of a vertex in a random graph has a binomial distribution $b(k ; n-1, p)$ with parameters $n-1$ and $p$. Based on this and the following constraints, he obtained the distribution of the degree sequence for a random graph.

Theorem 6.3.1 (Bollobas) Let $\epsilon>0$ be fixed, $\epsilon n^{-3 / 2} \leq p=p(n) \leq 1-\epsilon n^{-3 / 2}$, let $k=k(n)$ be a natural number, let $X_{k}$ be the number of vertices of degree $k$, and set $\lambda_{k}=\lambda_{k}(n)=n b(k ; n-1, p)$. Then, the following assertions hold.

i- If $\lim \lambda_{k}(n)=0$, then $\lim P\left(X_{k}=0\right)=1$

ii- If $\lim \lambda_{k}(n)=\infty$, then $\lim P\left(X_{k} \geq t\right)=1$ for every fixed $t$.

iii- If $0<\limsup \lambda_{k}(n) \leq \liminf \lambda_{k}(n)<\infty$

then $X_{k}$ has asymptotically Poisson distribution with mean $\lambda_{k}$ :

$$
P\left(X_{k}=r\right) \approx \frac{e^{-\lambda_{k}} \lambda_{k}^{r}}{r !}
$$

for every fixed $r$.

Proof: The proof of this theorem is beyond the scope of this study, see [180].

The conditions in the theorem hold when $n$ goes to infinity. For our problem we have $n \approx 2000$. Although $n$ is sufficiently large to find and approximate distribution, it is not enough to fulfill the constraints perfectly. We will see the affects of this while forming the actual (normalized) degree sequence histograms next.

For our problem $n \approx 2000, k \approx 20$, and $p<<1$. Therefore, we can use the Poisson approximation for the binomial distribution for the $\lambda_{k}$ (mean of the Poisson distribution for the number of vertices of degree $k$ ) as: 


$$
\lambda_{k}=n b(k ; n-1, p) \approx \frac{n e^{-\lambda_{p}} \lambda_{p}^{k}}{k !}
$$

where $\lambda_{p}=(n-1) p, \lambda_{p}$ is the mean of the Poisson distribution of $\lambda_{k}$ divided by $n$.

For our measure, we will need an estimate of the probability $p$, which captures the organization in a graph. To obtain $p$ from the distribution of $X_{k}$, we use the conditional expectation (conditional mean):

$$
E\left[E\left[X_{k} / \lambda_{k}\right]\right]=n \lambda_{p}=n(n-1) p
$$

By the conditional expectation in Eqn. 6.4 we are able to obtain a measure composed of the multiplication of $n(n-1)$ and $p$. Since both indicate the organization in the graph, their multiplication also serves as a measure for organization. Therefore, we will use the conditional mean as our next measure.

Before going further, let's consider four sample degree sequence distributions shown in Fig. 6.4. These images cover the full range of development. Our aim is to show that the normalized degree sequence histograms we obtain are very close to the Poisson distribution. In these figures, we give the normalized histogram of the degree sequence of each image and the best Poisson distribution fit. To fit the Poisson distribution, we search for the distribution mean that minimizes the squared error between the theoretical distribution and the histogram. In these figures, filled circles indicate the actual normalized histogram values and the empty circles represent the corresponding theoretical Poisson distribution values.

As can be seen, the normalized histograms and the Poisson distributions fit fairly well. This indicates that Bollobas' theorem applies reasonably well to our case (although $n$ does not go to infinity). Therefore, our first measure derived from the 


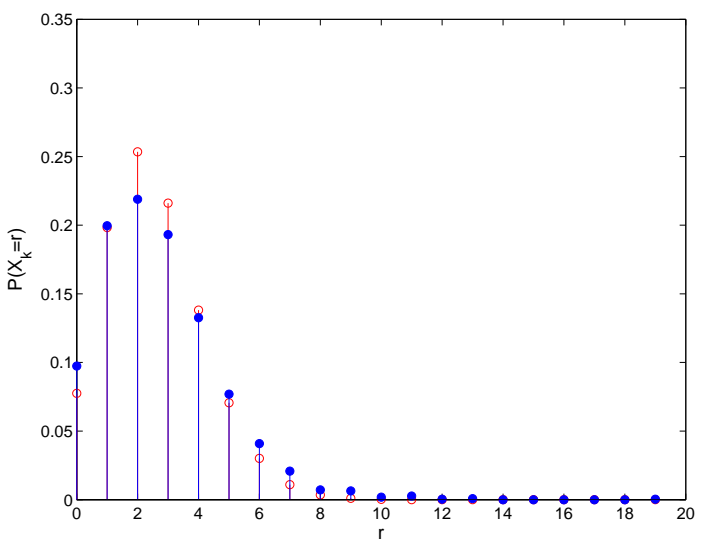

(a) The histogram and its Possion distribution fit for image 1

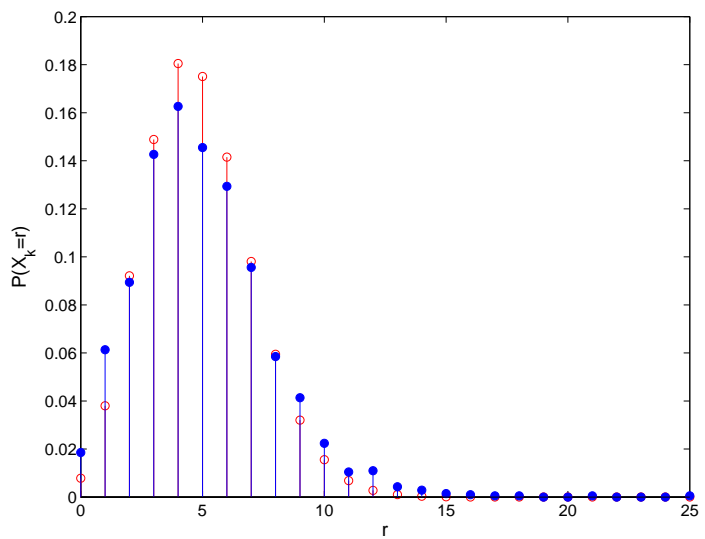

(c) The histogram and its Possion distribution fit for image 18

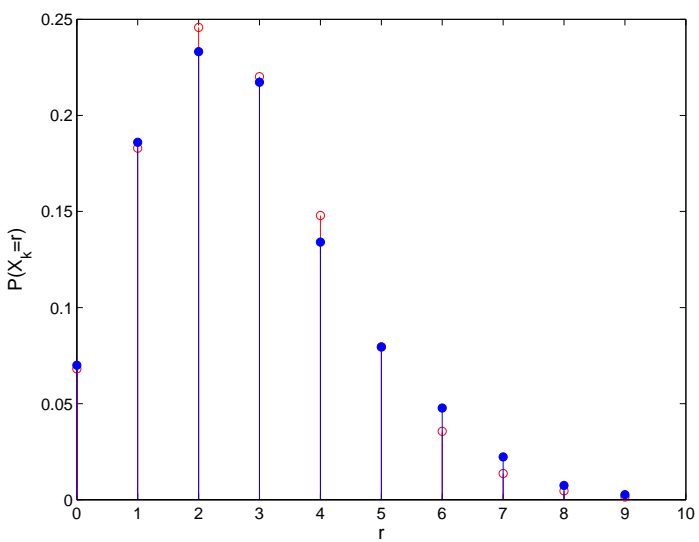

(b) The histogram and its Possion distribution fit for image 5

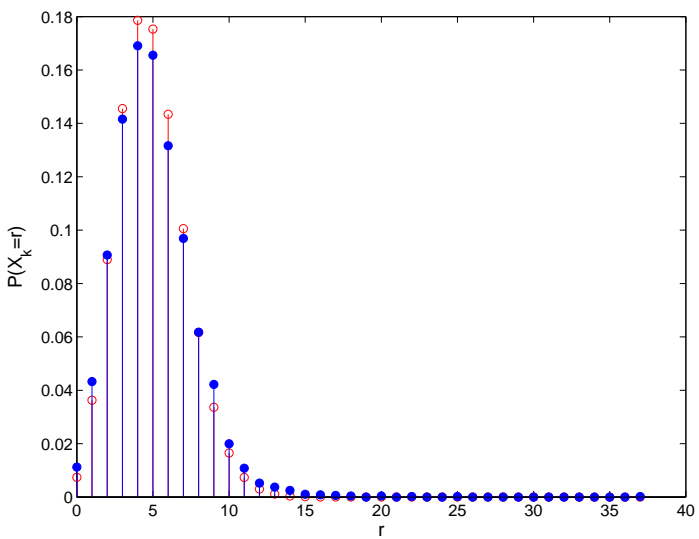

(d) The histogram and its Possion distribution fit for image 19

Figure 6.4: Sample degree sequence histograms and their best Poisson distribution fits. Filled circles - actual histograms, empty circles - Poisson distribution values. 
degree sequence $m_{d s f}$ is the mean of the Poisson distribution as calculated from a least squares fit.

Although this measure is reasonably informative, we experimentally observed that, if we estimate this mean by sample moments, we get even better results in terms of monotonicity. We can estimate the mean of the Poisson distribution by dividing the square of the first moment by the second central moment. This ratio simplifies to the mean for the Poisson distribution. Therefore, the second measure derived from the degree sequence $m_{d s}$ is:

$$
m_{d s}=\frac{\mu_{d e g}^{2}}{\sigma_{d e g}}
$$

This estimate of the mean works slightly better than the other. One reason for this may be that this calculation also accounts for fit errors (since we do not have an infinitely large $n$ required by the theorem), using all the data more effectively. Another benefit of this measure is that it does not require a search for the least squares fit to the Poisson distribution.

\subsection{Measures Based on Weighted Graphs}

Up to this point we considered unweighted graphs. In this section, we extend the development to weighted graphs. Given a graph $G=(V, E)$, suppose there is associated with each edge $e_{i j} \in E$ a positive number $w_{i j}$, called the weight of the

edge between vertex $i$ and $j$. The graph $G$, together with the function $w: E \rightarrow(0, \infty)$ is called a weighted graph.

Shapiro and Brady [181] defined the weight of an edge based on the attribute values of the vertices joined by that edge. Sarkar and Boyer [178] and Shi and Malik [182] 
defined similar weighting methods. In the same way, we define the weight of an edge $e_{i j}$ between two vertices $v_{i}, v_{j}$ to be:

$$
w_{i j}=\exp \left(\frac{-\beta\left(v_{i}, v_{j}\right)}{\alpha}\right)
$$

where $\beta\left(v_{i}, v_{j}\right)=\beta\left(v_{j}, v_{i}\right)$ is a function of the vertex attributes and $\alpha$ is a scale parameter.

Among the attributes assigned to our vertices, edge weighting according to length disparity captured the degree of organization most effectively. As development proceeds, and buildings (for instance) appear, the aspect ratios of those structures are generally not extreme. Thus, nearby line segments of similar length tend to associate with one another more commonly than nearby segments of highly different lengths. Basing the edge weights on the similarity in length between the two corresponding line segments captures this observation. So, we set $\beta\left(v_{i}, v_{j}\right)=\left|l_{i}-l_{j}\right|$ where $l_{i}$ is the length attribute of vertex $v_{i}$. After extensive testing, we settled on $\alpha=50$ as a suitable scale parameter. (Recall, ours are metric images, so changing scale is not an issue.)

The adjacency matrix of $G$ is the $n \times n$ matrix $\mathbf{A}=\left[w_{i j}\right]$. The eigenvalues and eigenvectors of graph $G$ are the eigenvalues and eigenvectors of $\mathbf{A}$. If $\lambda_{1}, \ldots, \lambda_{n}$ are the eigenvalues of $G$, the spectrum of the graph is defined as $s p(G)=\left[\lambda_{1}, \ldots, \lambda_{n}\right]$.

The degree matrix $\mathbf{D}$ is a diagonal matrix with $\sum_{j} w_{i j}$ of vertex $i$ at position $(i, i)$. The Laplacian matrix of the graph $G$ is defined as $\mathbf{L}=\mathbf{D}-\mathbf{A}$. The Laplacian matrix is explained in detail in [183]. 


\subsubsection{Graph Partitioning by the Laplacian Cut}

We next introduce three measures based on graph partitioning. All are based on a common idea: As development occurs, the number of edges in the graph (relative to the number of vertices) is expected to increase, as more linear structures in the image appear in mutual proximity. In rural regions, graph vertices are, to a great extent, isolated; indeed the graph may consist largely of small disjoint subgraphs. Therefore, we anticipate a few (large) vertex clusters in urban areas versus many (small) clusters in rural areas.

We obtain graph clusters by the Laplacian cut, based on the Laplacian matrix. Fiedler [184-186] introduced graph partitioning using the eigenvector corresponding to the second smallest eigenvalue of the Laplacian matrix. Actually, this eigenvector is called the Fiedler vector in the literature.

Before giving Fiedler's theorems on partitioning, we review other work using graph partitioning by eigenvectors from the computer vision literature. Sarkar and Boyer [178] used a graph partitioning method based on the adjacency matrix for change detection. Perona and Freeman [187] used a similar idea for image segmentation. Shi and Malik [182] introduced the normalized cuts method, based on the normalized Laplacian matrix, for image segmentation. Weiss [188] compared existing graph theory based segmentation algorithms. Gdalyahu et. al. [189] worked on image segmentation with a similar methodology. In recent work, Pavan and Pelillo [190] have developed a framework for image segmentation based on a new graph-theoretic

clustering formulation. They propose a novel combinatorial concept, the dominant set, that generalizes the notion of a maximal clique to weighted graphs. 
For our application the Laplacian cut is easy to compute and produces good results. Eigenclustering [178] may be considered a sort of "dual" approach (in that it groups, but does not cut per se). In the present case, it would be no faster to compute and no more informative. To be more specific, the Laplacian cut is 124 times faster on the average.

We next present Fiedler's theorems on graph partitioning. Fiedler defines the second smallest eigenvalue of the Laplacian matrix as the algebraic connectivity of the graph.

Theorem 6.4.1 (Fiedler) Let the second smallest eigenvalue $a(G)$ of the Laplacian graph $G, L(G)$, be called its algebraic connectivity. It satisfies

$$
a(G)=\min _{\mathbf{x} \in S} \mathbf{x}^{T} \mathbf{L}(G) \mathbf{x}
$$

where $S=\left\{\mathbf{x}=\left(x_{1}, \ldots, x_{n}\right)^{T} \in R_{n}, \sum_{i=1}^{n} x_{i}=0, \sum_{i=1}^{n} x_{i}^{2}=1\right\}, R_{n}$ is the space of all real column vectors with $n$ coordinates.

Proof: This follows immediately from the well-known Courant theorem since the smallest eigenvalue of $L(G)$ is zero and the corresponding eigenvector is e. Thus $S$ consists of all unit vectors of orthogonal to $e$, which means that $a(G)$ as the second smallest eigenvalue is the minimum of $\mathbf{x}^{T} \mathbf{L}(G) \mathbf{x}$ on $S$.

Fiedler also proved that a weighted connected graph can be partitioned into two connected subgraphs based on algebraic connectivity:

Theorem 6.4.2 (Fiedler) Let $G$ be a finite connected graph with $n$ vertices $1, \ldots, n$ to every edge $(i, k)$ of which a positive number $w_{i k}$ is assigned. Let $y=y_{i}$ be a characteristic valuation (eigenvector corresponding to the algebraic connectivity) of 
G. For any $r \geq 0$, let $M(r)=\left\{i \in N \mid y_{i}+r \geq 0\right\}$. Then the subgraph $G(r)$ induced by $G$ on $M(r)$ is connected.

Remark: A similar statement can be proved for $r \leq 0$ and the set $M^{\prime}(r)$ of all those $i$ 's for which $y_{i}+r \leq 0$.

Proof: The proof is beyond the scope of this study, see [186].

We form the weighted graphs to satisfy the constraints in the previous two theorems. We also define the Laplacian of a graph as Fiedler does. Therefore, Fiedler's theorems are directly applicable to our problem.

Although Fiedler did not give an optimal value of $r$ for partitioning, the median of the Fiedler vector has been used in the literature extensively for this purpose. In this study, we use this value to cut the graph into two parts. We apply the successive partitioning method suggested by Shi and Malik to obtain the final partitioning. The partitioning operation stops as the sum of the weights of edges to be cut exceeds a certain limit.

\section{The First Measure on Graph Partitioning}

The first of our measures based on graph partitioning is related to the size of the partitions (number of vertices in each). For well organized regions, we expect fewer clusters with more vertices in each. For rural regions, we expect more clusters with fewer vertices in each. As we checked the cluster count for graphs obtained from rural

regions (with sparse lines) we observed that the largest cluster has approximately 10 vertices on the average. Therefore, we define clusters having more than 10 vertices as large. The first measure $m_{l c 1}$ is the fraction of vertices appearing in large clusters. 


$$
m_{l c 1}=\frac{\sum_{i=1}^{K}|v c(i)|}{n}
$$

where $|v c(i)|$ is the number of vertices in cluster $i$ and $|v c(i)|>10$ for $i=1,2, \ldots, K$. $K$ is the number of large clusters and $n$ is the total number of vertices.

\section{Second and Third Measures on Graph Partitioning}

Our second and third measures combine structural (cluster) information with the unary attributes assigned to vertices. We take length and contrast attributes to form the measures $m_{l c 2}$ and $m_{l c 3}$ :

$$
\begin{aligned}
& m_{l c 2}=\frac{\sum_{i=1}^{n} l(i)}{N_{p}} \\
& m_{l c 3}=\frac{\sum_{i=1}^{n} c(i)}{N_{p}}
\end{aligned}
$$

where $N_{p}$ is the number of partitions, $l(i)$ is the length assigned to vertex $v_{i}$, and $c(i)$ the contrast assigned to vertex $v_{i}$. These measures reflect the observations that; as development ensues, line segments grow longer and exhibit greater contrast, while being grouped into a smaller member of (large) clusters.

\subsubsection{Singular Values of the Adjacency Matrix}

Our last two measures use the singular values of the adjacency matrix of a weighted graph $G$. The first measure is based on the quarter circularity of the distribution of the singular values; the second is based on the energy of the graph.

Since the adjacency matrix $\mathbf{A}$ is symmetric, its singular values are equal to the absolute values of its eigenvalues. Considering the size of our matrices, obtaining 
singular values is more robust (computationally stable) than obtaining eigenvalues. Therefore, we calculate the singular values instead of the eigenvalues.

\section{Circularity of the Singular Value Distribution}

Wigner [191] introduced "the semicircle law", proving that the eigenvalue distribution of a random symmetric matrix approaches a semicircular shape as the dimension of the matrix goes to infinity [191-193]. Arnold [194,195] extended Wigner's work and obtained the same law for deterministic symmetric matrices. His derivation of the semicircle law for the deterministic case is:

Theorem 6.4.3 (Arnold) Let $\mathbf{A}_{\mathbf{n}}=\left(a_{i j}\right), 1 \leq i, j \leq n$, be the $n$th section of

an infinite Hermitian matrix, $\left\{\lambda_{k}^{(n)}\right\}_{1 \leq k \leq n}$ its eigenvalues, and $\left\{u_{k}^{(n)}\right\}_{1 \leq k \leq n}$ the corresponding (orthonormalized column) eigenvectors. Let $v_{n}^{*}=\left(a_{n 1}, a_{n 2}, \ldots, a_{n, n-1}\right)$, put

$$
X_{n}(t)=[n(n-1)]^{-1 / 2} \sum_{k=1}^{(n-1) t}\left|v_{n}^{*} u_{k}^{n-1}\right|^{2}, 0 \leq t \leq 1
$$

(bookkeeping function for the length of the projections of the new row $v^{*}$ of $\mathbf{A}_{\mathbf{n}}$ onto the eigenvectors of the preceding matrix $\mathbf{A}_{\mathbf{n}-\mathbf{1}}$ ), and let finally

$$
F_{n}(x)=n^{-1} \text { (number of } \lambda_{k}^{(n)} \leq x \sqrt{n}, 1 \leq k \leq n \text { ) }
$$

(empirical distribution function of the eigenvalues of $\mathbf{A}_{\mathbf{n}} / \sqrt{n}$ ).

Suppose

$$
\begin{aligned}
& i-\lim _{n \rightarrow \infty} a_{n n}(t) / \sqrt{n}=0 \\
& i i-\lim _{n \rightarrow \infty} X_{n}(t)=C t \quad(0 \leq C \leq \infty, 0 \leq t \leq 1)
\end{aligned}
$$

Then

$\lim _{n \rightarrow \infty} F_{n}=W(\cdot, C)$

where $W$ is absolutely continuous with (semicircle) density 


$$
w(x, C)= \begin{cases}(2 C \pi)^{-1}\left(4 C-x^{2}\right)^{1 / 2} & |x| \leq 2 \sqrt{C} \\ 0 & |x|>2 \sqrt{C}\end{cases}
$$

Proof: The proof of this theorem is beyond the scope of this study, see [195].

Shen [196] generalized the semicircle law to singular values (absolute values of the eigenvalues). Instead of the semicircle, one obtains a quarter circle when considering singular values.

We apply this law to our adjacency matrices. Ideally this theorem holds as $n$ goes to infinity. For our practical application $n \approx 2000$, which is sufficient to observe similar characteristics. However, if the graph is sparse, we have two peaks around zero and one in the distribution in addition to the quarter circle shape. This is because of the sparseness of the adjacency matrix. Based on this observation and the semicircle law, our hypothesis for the first measure is that a graph obtained from rural regions (with sparse adjacency matrices) will have more sharply peaked singular value distributions than the less sparse city center distributions. We also expect a more quarter circle like shape for singular value distributions obtained from more organized regions.

To assess "quarter circularity" we calculate a functional fit error. Let the actual distribution (histogram) for the singular values be $f(\lambda)$. We approximate the circle function by a parabola $\hat{f}(\lambda)$ as:

$$
\hat{f}(\lambda)= \begin{cases}a+b \lambda+c \lambda^{2} & 0 \leq \lambda \leq 3 \\ 0 & \lambda>3\end{cases}
$$

where polynomial parameters $a, b$, and $c$ are obtained by the least squares fit. We can justify approximating the quarter circle function by a parabola as follows. First, as 
we check the distribution function in Eqn. 6.10, it is in fact an ellipse, a scaled circle (where we are using one half of it, proved by Shen). Also, Arnold's theorem holds as $n$ goes to infinity, however for our application we have $n \approx 2000$, which does not perfectly satisfy the requirements. We sometimes have sparse matrices, causing two dominant peaks around zero and one in the actual distribution function, besides the quarter circular shape. Finally, fitting a parabola to a function is easier than fitting an ellipse, and we are not after the exact shape for this measure. Therefore, fitting a parabola (closest to an elliptical shape) is reasonable to calculate our measure.

We give normalized histograms $f(\lambda)$ (solid curves) and the corresponding approximations $\hat{f}(\lambda)$ (dashed curves) of singular values $\lambda$ for four sample images in Fig. 6.5. These images cover the full range of development.

As can be seen, for the rural region (image 6) there are two dominant peaks around zero and one owing to the sparseness of the graph. The histograms of more organized regions (images 16 and 19) look more like quarter circles, as we expect. Farkas et. al. [197] obtained similar distributions for small world graphs $[198,199]$. Their aim was to analyze the graph structure by adding random edges successively.

The measure we propose based on quarter circularity is the negation of the fit error to singular value distributions:

$$
m_{f e}=-\|f(\lambda)-\hat{f}(\lambda)\|
$$

where $f(\lambda)$ is the distribution of singular values and $\hat{f}(\lambda)$ its approximation. In this formulation, the least squares fit also captures (inversely) the impact of the $(0,1)$ peaks on the overall distribution. As the actual distribution becomes more like a quarter circle, this measure increases. 


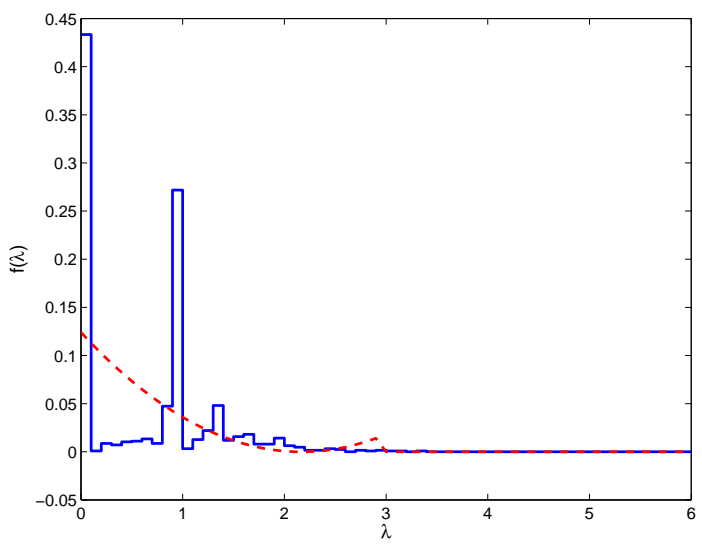

(a) $f(\lambda)$ and $\hat{f}(\lambda)$ for image 6

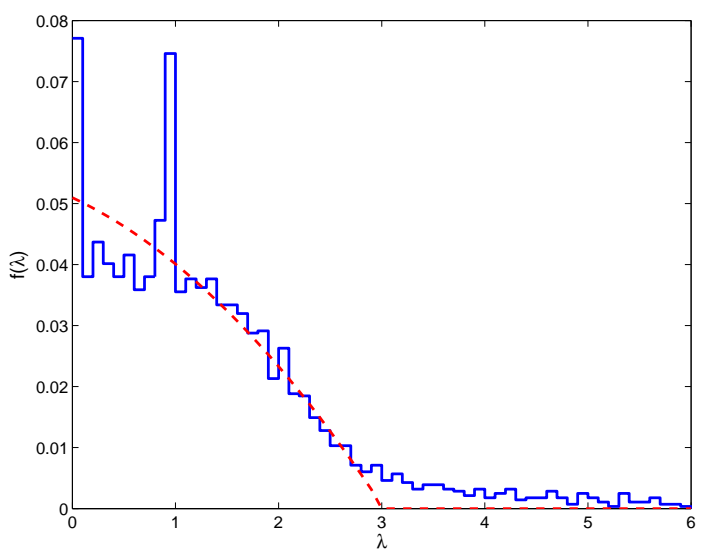

(c) $f(\lambda)$ and $\hat{f}(\lambda)$ for image 16

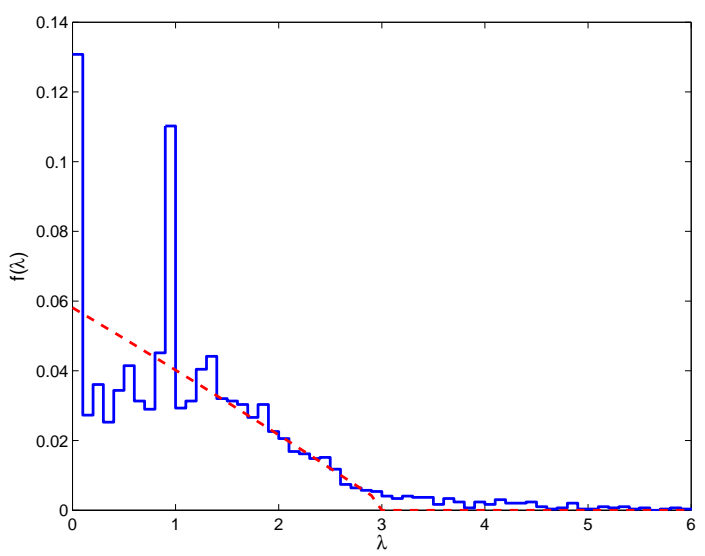

(b) $f(\lambda)$ and $\hat{f}(\lambda)$ for image 10

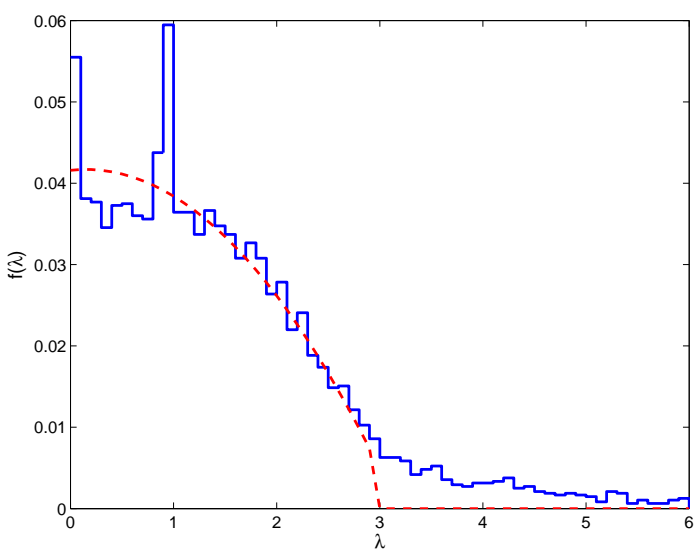

(d) $f(\lambda)$ and $\hat{f}(\lambda)$ for image 19

Figure 6.5: Normalized singular value distributions (solid curves) and corresponding approximations (dashed curves) for four sample images 


\section{The Unit Energy of a Graph}

The second singular value based measure is the unit energy, developed from the energy of a graph $E(G)$ as defined by Gutman and Polansky [200]:

$$
E=\sum_{j=1}^{n}\left|\lambda_{j}\right|
$$

They introduced this definition for graphs used in organic chemistry and it has roots in the chemical properties of molecules. Cvetkovic et. al. [201] followed the same definition for general graphs.

Based on this energy definition, the unit energy measure, or energy per vertex, is:

$$
m_{\text {ueg }}=\frac{E}{n}
$$

We expect the unit energy of a graph to increase as the organization in it increases.

Although we tested total energy as a measure, unit energy outperformed it experimentally. The unit energy of the graph can also be taken as the sample mean of the singular values. This observation relates our second measure on singular values to the first.

\subsection{Fusing Measures}

Since we have several measures, and each is based on different properties of the graph, we fuse them to improve performance. We first normalize each measure to $[0,1]$. We then define a hard limiter function such that new samples may not exceed this range. We fuse the normalized measures by taking their median. To make this definition more specific, let's assume image $i$ yields (normalized) measures $m_{c r}(i)$, 
$m_{d s}(i), m_{l c 1}(i), m_{l c 2}(i), m_{l c 3}(i), m_{f e}(i)$, and $m_{\text {ueg }}(i)$. We take the median of these values and assign it to the fusion measure $m_{F}(i)$ for image sample $i$.

\subsection{Experimental Results}

We now present two sets of experiments on these measures. The first experiment compares them with a (collective) human assessment of organization. This is to evaluate their monotonicity. The second set of experiments evaluates the discrimination power of them in extracting residential regions.

\subsubsection{Monotonicity: Comparison to Manual Evaluations}

To compare these measures to human assessment of organization, we selected 20 images (Fig. 6.1) covering the range from (effectively) wilderness to dense urban. We note that humans are inconsistent in ranking the least developed areas - there were six such images among the 20 - so a comparison against our measures would be meaningless for these six (and we have other, direct measures to eliminate decidedly rural areas given in chapter 3). For the remaining 14 images, the 90 people demonstrated reasonable consensus - but not unanimity. For comparison, we define two types of error: deviation and false alarm. We define the deviation for each image to be $1 / 2$ of the absolute difference (to avoid double counts) in the ordinal position (1 through 14) as assigned by the measure versus that of the human consensus. The sum of the per-image deviations over the set provides the score for the measure.

Since we are also interested in distinguishing rural from non-rural images, we define a false alarm to be the event in which a rural image (by human classification) is assigned a ranking above the lowest non-rural image. The consequence of a false alarm 
in practice is nonfatal; it simply means that we will expend additional computation on a region that will ultimately prove uninteresting.

Table 6.2 shows the deviation and false alarm counts with respect to the consensus ordering. The fused measure $m_{F}$ presents the best performance with respect to deviation; $m_{l c 3}$ provides the best performance with respect to the false alarm rate.

\begin{tabular}{|l|l|c|c|}
\hline Measure & Origin & False alarms & Deviation \\
\hline$m_{c r}$ & circuit rank & 3 & 6 \\
\hline$m_{d s f}$ & degree sequence (l.s. fit) & 4 & 5 \\
\hline$m_{d s}$ & degree sequence (moments) & 4 & 4 \\
\hline$m_{l c 1}$ & first partition measure & 4 & 5 \\
\hline$m_{l c 2}$ & second partition measure & 3 & 4 \\
\hline$m_{l c 3}$ & third partition measure & 2 & 4 \\
\hline$m_{f e}$ & distribution of singular values & 4 & 3 \\
\hline$m_{u e g}$ & unit energy of a graph & 3 & 4 \\
\hline$m_{F}$ & fusion of measures & 4 & 2 \\
\hline
\end{tabular}

Table 6.2: False alarms and deviations for measures

In Fig. 6.6 we plot each measure versus consensus ordering for all 20 images, including the first six for which the humans exhibited no real consensus. If there were perfect agreement between the consensus ranking and a measure, its curve would be monotonic. Because we are most interested in non-rural regions, it is really the monotonicity for images 7 through 20 that matter. Over this range almost all of our measures perform fairly well. Notably, $m_{F}$ performs extremely well and the deviations observed for images 14 and 15 are understandable in light of their similar $m_{F}$ values.

It is instructive to consider the appearance of those "high scoring" images among the first six. These are forested regions showing phantom structure due to shadows. In these images, the sun provides unidirectional illumination imposing a fairly regular 


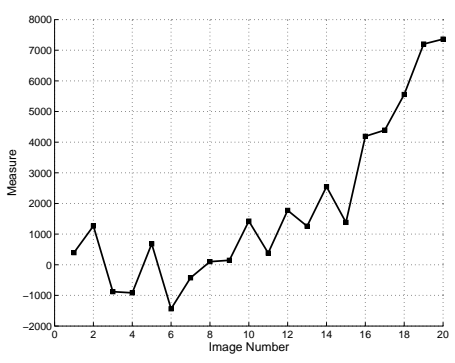

(a) Ordering by the circuit rank measure $\left(m_{c r}\right)$

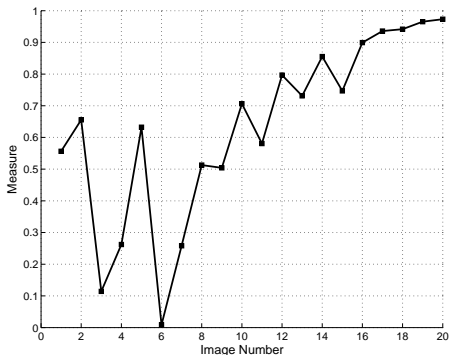

(d) Ordering by the first partition measure $\left(m_{l c 1}\right)$

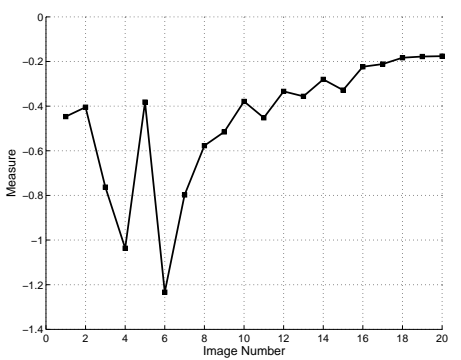

(g) Ordering by the distribution of singular values measure $\left(m_{f e}\right)$

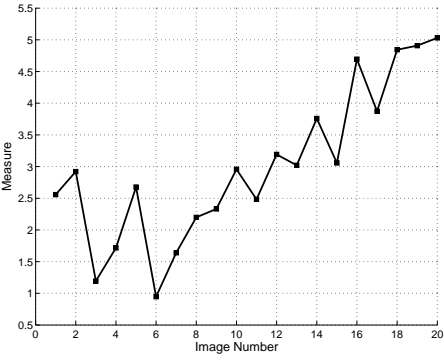

(b) Ordering by the degree sequence measure (l.s. fit) $\left(m_{d s f}\right)$

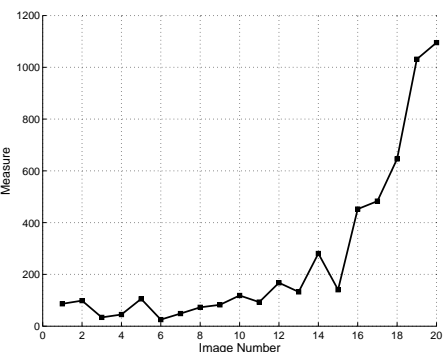

(e) Ordering by the second partition measure $\left(m_{l c 2}\right)$

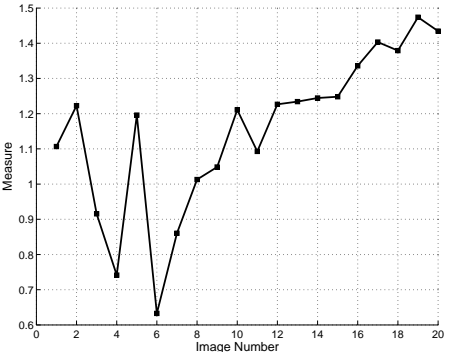

(h) Ordering by the unit energy of a graph measure $\left(m_{\text {ueg }}\right)$

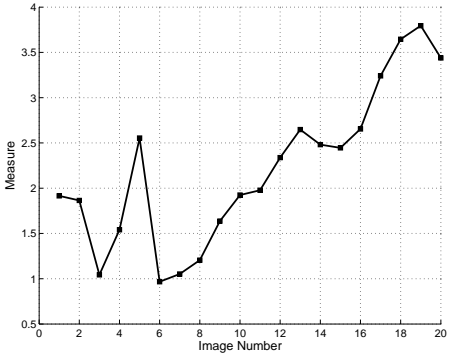

(c) Ordering by the degree sequence measure (moments) $\left(m_{d s}\right)$

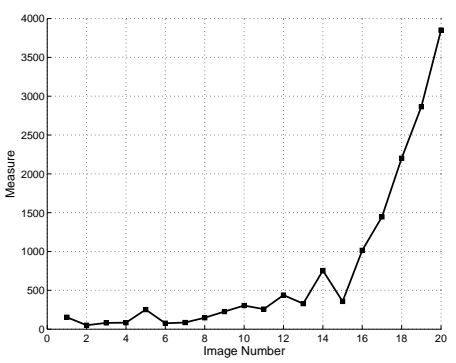

(f) Ordering by the third partition measure $\left(m_{l c 3}\right)$

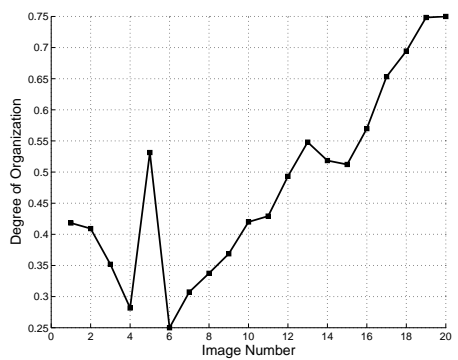

(i) Ordering by the fusion of measures $\left(m_{F}\right)$

Figure 6.6: Ordering of sample images based on all of our measures 
photometric structure on the image. Therefore, these images in fact seem as regular as residential regions. As we noted before, residential regions have forest characteristics. Fortunately, we can eliminate these in a preclassifier based on feature density (primarily) reported in chapter 3. While those features work well in eliminating rural regions, they have proven less effective in discriminating among levels of (sub)urban development, hence the current work.

\subsubsection{Comparison with Sarkar and Boyer's Measures}

The measures of Sarkar and Boyer, used for change detection, are perhaps the closest to ours to be found in the literature. In this section, we compare these measures with those proposed in this study. For completeness we summarize their measures below.

Sarkar and Boyer define their graphs on constant curvature edge segments in the image (unlike our straight lines). They also obtain the weights in the graph by Gestalt-inspired relationships between vertices.

Instead of partitioning the graph as we did, they obtain eigenclusters from the eigenvectors of the adjacency matrix. An eigencluster is the the collection of nodes

corresponding to the nonzero components of a positive eigenvector $\left(\mathbf{x}_{\mathbf{i}}\right.$, such that all the components of $\mathbf{x}_{\mathbf{i}}$ or $-\mathbf{x}_{\mathbf{i}}$ are positive and its corresponding eigenvalue is positive). They define their measures on these eigenclusters.

Their first measure is $I_{\text {tot }}^{c}$ :

$$
I_{\text {tot }}^{c}=\frac{\sum_{i=1}^{N c}\left(\sum_{j=1}^{n i} l_{i j}\right)}{N_{c}} N
$$


where $l_{i j}$ is the length of the $j$ th edge segment in the $i$ th eigencluster, $N$ is the total number of edge segments, and $N_{c}$ is the total number of eigenclusters. This gives the ratio of the the total length of segments times total number of edge segments divided by the total number of clusters.

Their second measure is the total number of eigenclusters $N_{c}$. For our purposes, the fewer eigenclusters a graph has, the more organized it is. Therefore, we take the negation of this measure in our comparison.

Their last measure is the sum of the positive eigenvalues $\lambda_{+}$. This corresponds to half of the energy $E$, as given in Eqn. 6.13 [166].

To compare these measures with ours, we implemented the eigenclustering algorithm on our weighted graphs. Therefore, a one to one comparison is not possible. However, we give performance their measures here to show how they behave in our domain. We first give the false alarm and deviation counts for these measures in Table 6.3.

\begin{tabular}{|l|c|c|}
\hline Measure & False alarms & Deviation \\
\hline$I_{\text {tot }}^{c}$ & 4 & 6 \\
\hline$-N_{c}$ & 1 & 14 \\
\hline$\lambda_{+}$ & 4 & 10 \\
\hline
\end{tabular}

Table 6.3: False alarms and deviations for Sarkar Boyer measures

As can be seen, these measures are not as powerful as ours in our domain. In Fig. 6.7 we plot Sarkar and Boyer's measures versus consensus ordering for all 20 images. Again, their monotonicity properties are not as good as ours. In Fig. 6.7 we plot Sarkar and Boyer's measures versus consensus ordering for all 20 images. 


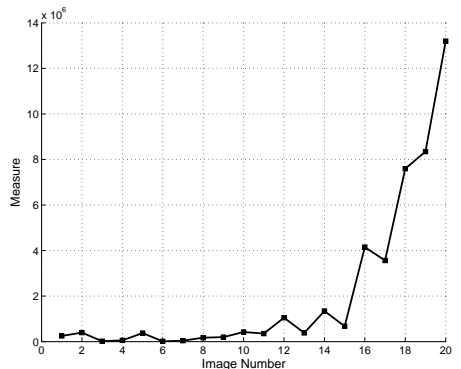

(a) Ordering by $I_{t o t}^{c}$

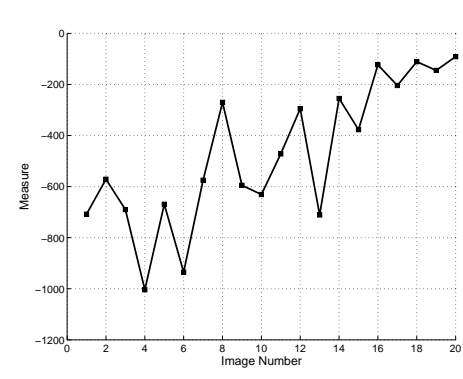

(b) Ordering by $-N_{c}$

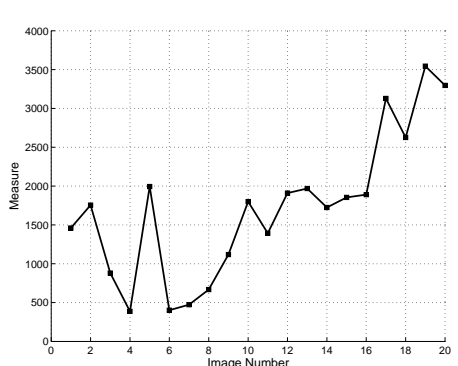

(c) Ordering by $\lambda_{+}$

Figure 6.7: Ordering of sample images based on three Sarkar Boyer measures

Again, their monotonicity properties are not as good as ours. These results also suggest that the $-N_{c}$ measure can also be used to eliminate the first six images besides the contrast measures.

\subsubsection{Detecting Residential Regions}

Our next set of tests uses the new measures in a detection problem: identifying sparse residential regions. This problem is both of particular importance, and of particular difficulty, as described above. In this section, we also use the structural features (given in chapter 3) both for comparison and in a combined feature space for improved overall results.

We have 295 nonoverlapping images taken from different parts of the US including representatives from a wide range of regions, climates, and terrain. We first consider the problem of detecting residential regions only (i.e. residential vs. nonresidential). Then we recast the problem as three way classification into rural, residential, and urban. To form the ground truth, we take the consensus ordering (Fig. 6.3) as a 
benchmark, then we label all the test images by hand in accordance with the consensus.

Before giving the one and three class classification results, let's look at some 2D histograms of our measures in Fig. 6.8. In these histograms we label rural, residential, and urban distributions with different colors (blue corresponds to rural, black corresponds to residential, and red corresponds to urban distributions). For each figure, the $y$ axis corresponds to the first measure and the $x$ axis corresponds to the second measure given in the caption.

As the histograms show, residential region distributions lie between those of rural and urban regions, as is the case for these regions in real space. Residential regions can be separated easily in spaces spanned by these graph based measures. In the structural feature space, residential regions overlap more with rural and urban regions. We will see the effect of this overlap in the following sections. Besides, in most of the feature spaces, urban regions have sub-clusters. This may be beneficial if higher levels (more specific) of classification are desired.

These histograms also indicate that using a Bayes classifier is not unreasonable in these spaces. Although the class distributions are not truly Gaussian, the urban samples being the worst case, they are reasonably close to be approximated as Gaussians.

\section{One Class Problem}

Here, we formulate the problem using only residential region training samples. We begin by estimating the mean vector $\mu_{\mathbf{x}}$ and covariance matrix $\boldsymbol{\Sigma}_{\mathbf{x}}$ of the residential region class in feature space assuming a bivariate Gaussian distribution. We then 


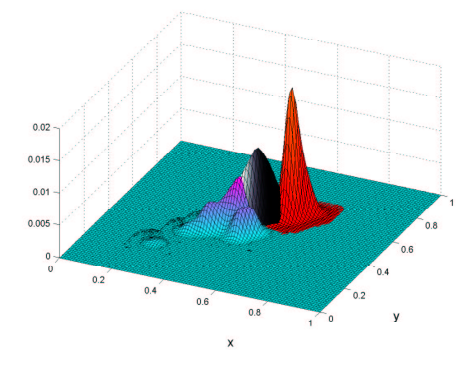

(a) Histogram for $\mu_{c}, E_{c}$

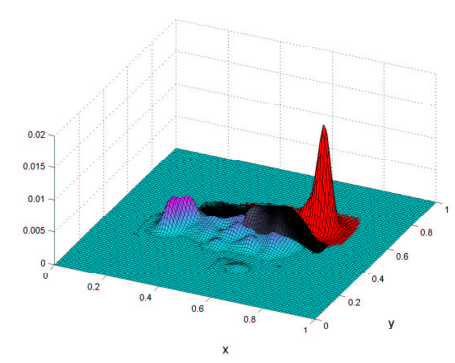

(d) Histogram for $E_{c}$, $m_{l c 1}$

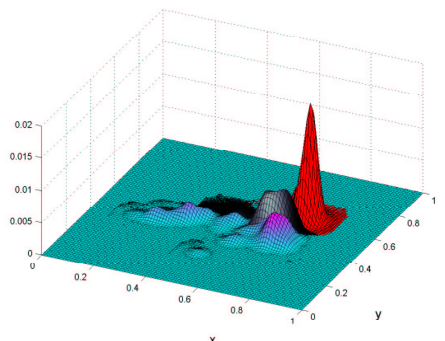

(g) Histogram for $E_{c}$, $m_{f e}$

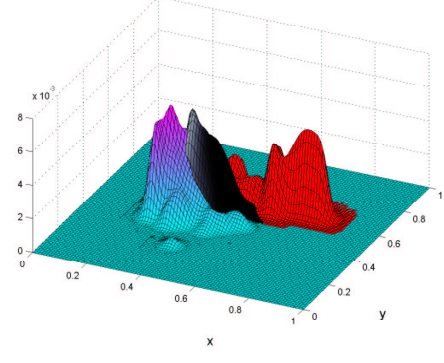

(b) Histogram for $E_{c}$, $m_{c r}$

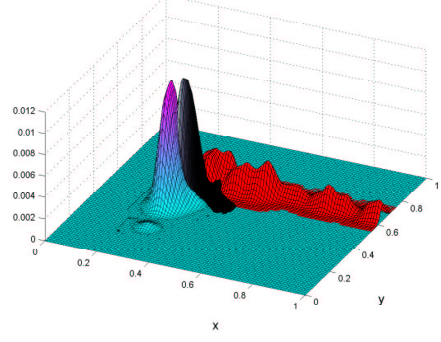

(e) Histogram for $E_{c}$, $m_{l c 2}$

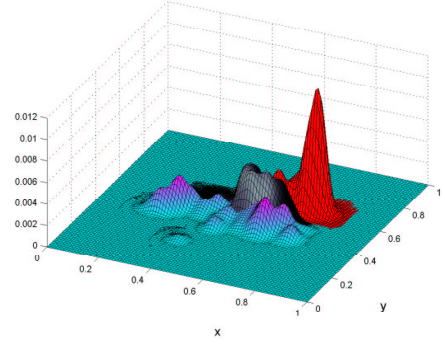

(h) Histogram for $E_{c}$, $m_{\text {ueg }}$

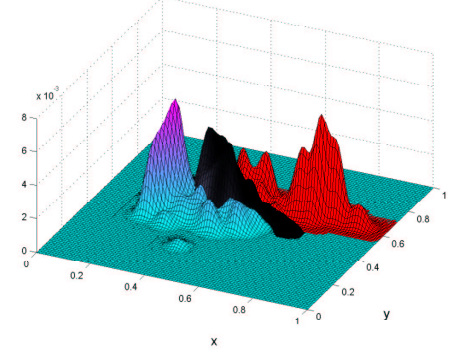

(c) Histogram for $E_{c}$, $m_{d s}$

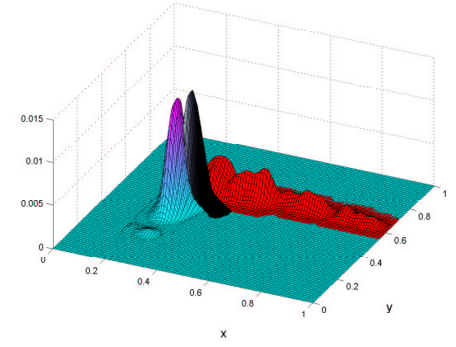

(f) Histogram for $E_{c}$, $m_{l c 3}$

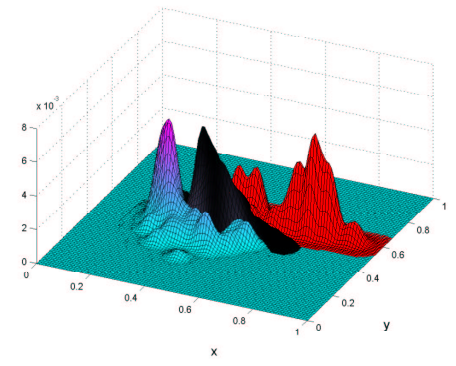

(i) Histogram for $E_{c}, m_{F}$

Figure 6.8: Histograms in 2D spaces, blue - rural region, black - residential region, red - urban region 
compute the Mahalanobis distance as given in Eqn. 3.16 between each window to be classified and this distribution:

The relative probability of a feature vector $\mathbf{x}$ to be a residential region is then computed as:

$$
\mathcal{M}=e^{-\frac{D_{M}}{10^{-3}}}
$$

where the normalization factor $10^{-3}$ is comparable with the Mahalanobis distances observe.

We use a Neyman-Pearson decision rule. We tested 91 residential and 190 nonresidential samples for this case. We train our classifier by four representative residential region samples, such that each correspond to a distinct residential region type. We give the receiver operating characteristics (ROC) curves for the various two dimensional feature spaces in Fig. 6.9. In these spaces, one dimension corresponds to the entropy of contrast given in Eqn. 3.15, $\left(E_{c}\right)$, which works well in separating rural and non-rural regions; the second dimension corresponds to one of the graph based measures, which work well in distinguishing urban and residential regions. To compare our results with the structural feature space alone $\left(\mu_{c}, E_{c}\right)$, we also include its ROC curve (dashed plots). We also tested spaces of more than two dimensions, but they perform no better.

We also tabulated the area under ROC curves for each feature space separately in Table 6.4. The area under a ROC curve gives a rough idea about the performance of that feature space on the average. The maximum that can be attained is one for the area under a ROC curve, which corresponds to a perfect case. 


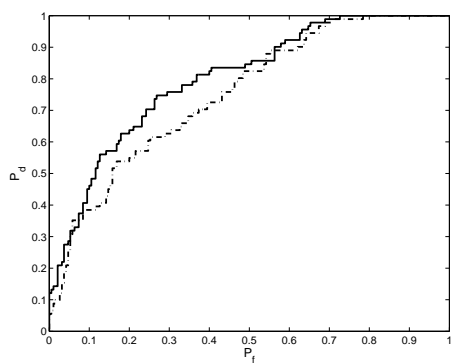

(a) $E_{c}, m_{c r}$

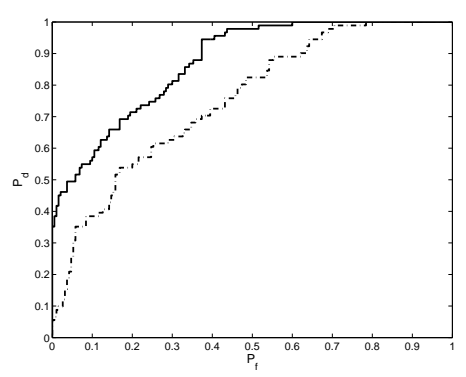

(d) $E_{c}, m_{l c 2}$

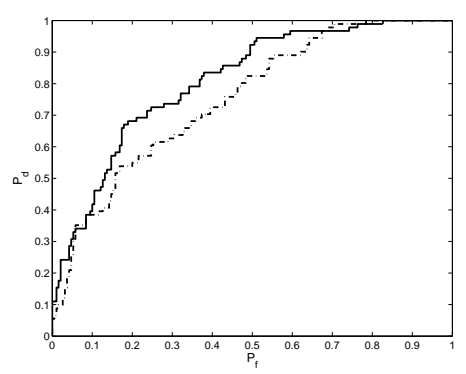

(g) $E_{c}, m_{\text {ueg }}$

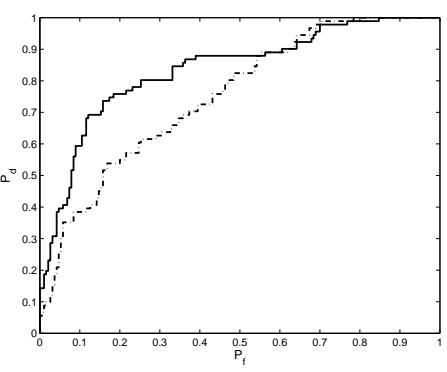

(b) $E_{c}, m_{d s}$

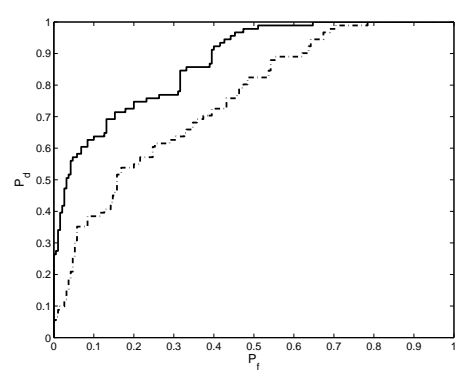

(e) $E_{c}, m_{l c 3}$

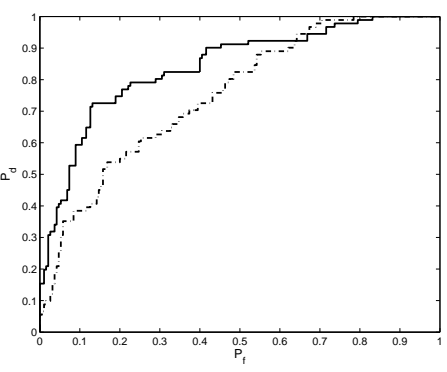

(h) $E_{c}, m_{F}$

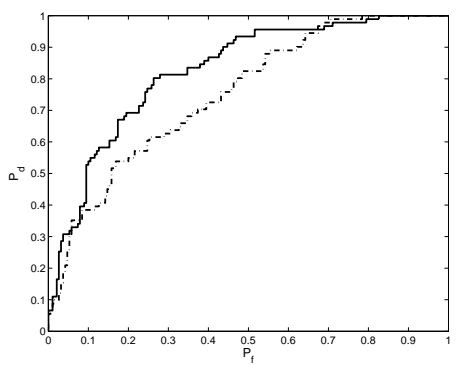

(c) $E_{c}, m_{l c 1}$

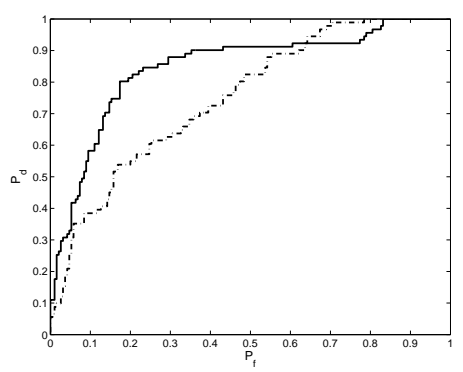

(f) $E_{c}, m_{f e}$

Figure 6.9: ROC curves for residential region detection 


\begin{tabular}{|l|c|}
\hline Feature space & Area \\
\hline$E_{c}, \mu_{c}$ & 0.7476 \\
\hline$E_{c}, m_{c r}$ & 0.7937 \\
\hline$E_{c}, m_{d s}$ & 0.8318 \\
\hline$E_{c}, m_{l c 1}$ & 0.8257 \\
\hline$E_{c}, m_{l c 2}$ & 0.8659 \\
\hline$E_{c}, m_{l c 3}$ & 0.8677 \\
\hline$E_{c}, m_{f e}$ & 0.8428 \\
\hline$E_{c}, m_{\text {ueg }}$ & 0.8061 \\
\hline$E_{c}, m_{F}$ & 0.8370 \\
\hline
\end{tabular}

Table 6.4: Area under ROC curves

As can be seen, partition based measures preformed fairly well in terms of areas under ROC curves. However, as we mentioned this only represents a rough idea about average performance of the feature space. The ROC curves obtained from the space using a graph based measure are better than the space using only structural features. In particular, $\left(E_{c}, m_{d s}\right)$ performs best at low false alarm rates while $\left(E_{c}, m_{l c 2}\right)$ and $\left(E_{c}, m_{l c 3}\right)$ perform fairly well for false alarm rates around 0.5 .

\section{Three Class Problem}

We next construct a Bayes classifier, assuming equal prior probabilities, labelling each image as rural, residential, or urban. We tested the classifier on 90 rural, 89 residential and 91 urban samples. We used five rural, four residential, and six urban representative samples to train the classifier. Our training samples again correspond to distinct region types in each class.

We discard the circuit rank measure $m_{c r}$ in calculating the $m_{F}$ for three class problem. Although it served in fusion for a two class case, it decreased the performance here. Therefore, we discarded it in calculating $m_{F}$. Since two class and three class 
settings are different, it is reasonable for a measure to perform good in one setting and poor in the other.

Classification results on structural features alone appear in Table 6.5. The overall correct classification rate for this space is $75.2 \%$. Next, we tabulate the best structural and graph theoretical measures after an exhaustive search over the full feature set (all singles, pairs, triples, etc.. The first feature space is $E_{c}, m_{d s}$ with a $83.0 \%$ overall classification rate (Table 6.6 ). The next feature space is $\mu_{c}, m_{d s}$ with a $80.4 \%$ overall classification rate (Table 6.7 ). The final feature space is $\mu_{c}, m_{F}$ with a $74.8 \%$ overall classification rate (Table 6.8).

\begin{tabular}{|l|c|c|c|}
\hline \multirow{2}{*}{} & \multicolumn{3}{|c|}{ percentage classified as } \\
\cline { 2 - 4 } & rural & residential & urban \\
\hline 90 rural samples & 75.6 & 23.3 & 1.1 \\
\hline 91 residential samples & 16.5 & 71.4 & 12.1 \\
\hline 89 urban samples & 0.0 & 21.3 & 78.7 \\
\hline
\end{tabular}

Table 6.5: Three class classification results on $\mu_{c}, E_{c}$ space

\begin{tabular}{|l|c|c|c|}
\hline \multirow{2}{*}{} & \multicolumn{3}{|c|}{ percentage classified as } \\
\cline { 2 - 4 } & rural & residential & urban \\
\hline 90 rural samples & 97.8 & 0.0 & 2.2 \\
\hline 91 residential samples & 13.2 & 78.0 & 8.8 \\
\hline 89 urban samples & 0.0 & 27.0 & 73.0 \\
\hline
\end{tabular}

Table 6.6: Three class classification results on $E_{c}, m_{d s}$ space 


\begin{tabular}{|l|c|c|c|}
\hline \multirow{2}{*}{} & \multicolumn{3}{|c|}{ percentage classified as } \\
\cline { 2 - 4 } & rural & residential & urban \\
\hline 90 rural samples & 85.6 & 13.3 & 1.1 \\
\hline 91 residential samples & 6.6 & 82.4 & 11.0 \\
\hline 89 urban samples & 0.0 & 27.0 & 73.0 \\
\hline
\end{tabular}

Table 6.7: Three class classification results on $\mu_{c}, m_{d s}$ space

\begin{tabular}{|l|c|c|c|}
\hline \multirow{2}{*}{} & \multicolumn{3}{|c|}{ percentage classified as } \\
\cline { 2 - 4 } & rural & residential & urban \\
\hline 90 rural samples & 73.3 & 26.7 & 0.0 \\
\hline 91 residential samples & 5.5 & 89.0 & 5.5 \\
\hline 89 urban samples & 9.0 & 29.2 & 61.8 \\
\hline
\end{tabular}

Table 6.8: Three class classification results in $\mu_{c}, m_{F}$ space

Comparing the results, we see that the classifiers including graph based measures have higher residential region detection rates (lower miss probabilities) than the classifier using structural features alone. Among them, the classifier using the $\mu_{c}, m_{F}$ space performs best in terms of high residential region detection rate, 89.0\%. Although the urban region detection rate decreased in this classifier, our primary goal is to improve the residential region detection rate.

The classifier using the $\mu_{c}, m_{d s}$ space has fairly low false alarm rate and a residential region detection rate close to the classifier using the $\mu_{c}, m_{F}$ space. In this feature space, the structural feature $\mu_{c}$ seems to dominate; such that rural and the rest is discriminated with high performance, but most of the residential samples are classified as urban. This was also the case in the previous chapters. 
We also observe that the classifier using the $E_{c}, m_{d s}$ space yields the highest rural region detection rate. In this feature space, the structural feature $E_{c}$ seems to dominate, similar to the previous space.

As for the $\mu_{c}, m_{F}$ feature space, the classifier decides in favor of the residential class. Therefore, it has the highest residential region detection rate. Depending on the application, this may be desirable or not. For our subsequent processing it is desirable, since the cost of misses is more than the cost of false alarms. However, depending on the cost assignments, we can select the appropriate feature space among these.

To see the improvement in residential region classification, in Table 6.9 we give the difference $(\Delta)$ between Tables 6.8 and 6.5. A negative sign in a diagonal entry indicates a decrement in classification performance in moving from the "original" $\mu_{c}$, $E_{c}$ space to the $m_{c}, m_{F}$ space. The urban region detection rate decreased using the graph based measures. Similarly, a negative sign in a non-diagonal entry indicates improvement. There is a clear improvement in residential region classification.

\begin{tabular}{|l|c|c|c|}
\hline \multirow{2}{*}{} & \multicolumn{3}{|c|}{ percentage classified as } \\
\cline { 2 - 4 } & rural & residential & urban \\
\hline 90 rural samples & -2.2 & 3.3 & -1.1 \\
\hline 91 residential samples & -11.0 & 17.6 & -6.6 \\
\hline 89 urban samples & 9.0 & 7.9 & -17.0 \\
\hline
\end{tabular}

Table 6.9: $\Delta$ classification on $\left(\mu_{c}, m_{d s}\right)$ - classification on $\left(\mu_{c}, E_{c}\right)$ 


\subsection{Summary of the Chapter}

In this chapter, we introduced and tested several graph theoretical measures (defined on both unweighted and weighted graphs) to assess land development in overhead imagery. We fused these measures to obtain a more monotonic (well-behaved) measure. Our first objective was to develop measures that increase monotonically with respect to the organization (development) in images. Our second objective was detecting sparse residential regions, which are difficult to discriminate. Next, we will detect houses and street networks in these. 


\section{CHAPTER 7}

\section{House and Street Network Detection in Residential Regions}

In the previous chapters, we introduced statistical measures to extract residential regions. As a step toward automatic map generation, we introduce a novel system to detect houses and street networks in residential regions here.

Detecting houses is far more challenging than detecting larger buildings for several reasons. First, their relatively small size (in one meter resolution images) makes their detection difficult. Second, occlusion by nearby trees is common. Third, in some neighborhoods, houses may come in fairly complex shapes. Analogous problems (small cross section, overhanging trees, and winding curves) present challenges for street detection in residential regions.

Our house and street detection system comprises four main parts. First, we introduce measures on multispectral images to detect regions of possible human activity. On these measures, we introduce a variation of the k-means clustering algorithm to extract possible houses and street networks by combining both spatial and spectral features. This combination of information improves the final clustering results. From clustering, we obtain a binary image containing possible street network fragments

and houses. We then decompose this binary image using a balloon algorithm based 
on binary mathematical morphology. Having obtained the decomposition, we represent them in a graph theoretical framework. Balloons serve as vertices and their neighborhood information is encoded as edges in the graph. The street network is extracted from the graph by using the shortest paths algorithm. The remaining vertices (balloons) are assigned as possible houses in the region.

We tested our system on a large and diverse data set. Our results indicate the usefulness of our system in detecting houses and street networks, hence generating automated maps.

\subsection{Prior Work}

In this section, we briefly review the literature on road and house detection. We group previous studies as follows: building detection (alone), road detection (alone), and combined building and road detection. The last group of studies exploits the mutual information between buildings and road networks to improve detection.

\subsubsection{Building Detection}

Mayer [202] surveyed object detection systems from aerial images, focusing on building detection. In his excellent survey, he classified building detection systems based on their complexities (in data, building model, and system strategy). Following Mayer's format (in simplified form), we add missing and new papers published after this survey in Table 7.1. Besides his performance measures, Shufelt's paper [203] also offers another survey on aerial building detection.

In Table 7.1, data complexity summarizes the resolution of the input image, relative location of the buildings, and the complexity of the scene. As for image types, 


\begin{tabular}{|l|c|c|c|}
\hline Approach & Data & Model & Strategy \\
\hline Huertas and Nevatia [204] & low-medium & simple & medium \\
\hline Shufelt and McKeown [205] & low-medium & medium & medium \\
\hline Krishnamachari and Chellappa $[206]$ & low-medium & simple & medium \\
\hline Maloof et al. $[207]$ & low-medium & medium & medium \\
\hline Brunn and Weidner [208] & high & medium & high \\
\hline Zhang [209] & high & simple & simple \\
\hline Kim and Muller $[210]$ & low & simple & medium \\
\hline Stassopoulou and Caelli $[211]$ & low & medium & high \\
\hline Gamba et al. $[212]$ & high & medium & simple \\
\hline Noronha and Nevatia $[213]$ & low & simple & high \\
\hline Fradkin et al. $[214]$ & medium & high & medium \\
\hline Krishnamoorthy et al. $[215]$ & medium & medium & medium \\
\hline
\end{tabular}

Table 7.1: Building detection review, in Mayer's format

some researchers used satellite images (such as Landsat, SPOT, and IRS) with resolutions in the 5 to 30 meter range. Some researchers used aerial images with resolutions in the 0.3 to 1 meter range. Some used Digital Elevation Map (DEM) or Digital Surface Model (DSM) data. Some others used Synthetic Aperture Radar (SAR) images. Each of these representations has benefits and shortcomings of its own.

Model complexity summarizes the building model used. It may be a simple 2D rectangle, a 2D polynomial, or a 3D surface. The model should serve the system's application. If only detection is required, a simple rectangle as a building model may suffice. However, if a detailed site model is required, 3D surface models as well as polynomial representations are necessary.

System strategy summarizes the complexity of the system. Perceptual organization, Bayesian networks, and graph theoretical methods are some of the approaches used in building detection systems. 
Neither Mayer nor Shufelt provided the classification performances of the systems they reviewed. Here, we fill this gap by providing reported classification performances in Table 7.2. In accordance with Lin and Nevatia [216], we use probability of detection and branching factor to report the performances. Probability of detection $P_{d}$ is the percentage of the ground truth buildings detected. Branching factor $B_{f}$ is the ratio of number of non-building objects labelled as buildings to the number of objects labelled as buildings.

\begin{tabular}{|l|r|r|}
\hline Approach & $\mathbf{P}_{\mathbf{d}}(\%)$ & $\mathbf{B}_{\mathbf{f}}(\%)$ \\
\hline Shufelt and McKeown [205] & $49.8-86.6$ & $12.8-32.2$ \\
\hline Maloof et al. $[207]$ & $90.0-91.6$ & \\
\hline Lin and Nevatia [216] & 71.9 & 6.7 \\
\hline Collins et al. $[217]$ & 89.0 & 46.0 \\
\hline Kim and Muller [210] & 76.3 & \\
\hline Stassopoulou and Caelli [211] & 97.6 & 12.0 \\
\hline Noronha and Nevatia [213] & 96.4 & 0.0 \\
\hline Fradkin et al. $[214]$ & 80.3 & 1.0 \\
\hline Krishnamoorthy et al. $[215]$ & $41.5-86.9$ & \\
\hline
\end{tabular}

Table 7.2: Building detection review, performances

For the systems reported, $P_{d}$ varies from $41.5 \%$ to $97.6 \%$, with $B_{f}$ in the $0.0 \%$ to $46.0 \%$ range. The performance depends on the resolution of the image, the density of buildings in the scene, and finally the size of the buildings to be detected. It is also important to remember that in most of these papers the ground truth has not been reported in detail, such as the number and size of the buildings. Therefore, a reliable comparison is not feasible.

The size of the buildings to be detected affects the performance. For example, Kim and Muller [210] reported $P_{d}=79.1 \%$ for house detection over 12 houses which 
is far less than the maximum $P_{d}$ obtained. Krishnamoorthy et al. [215] observed that most of their errors originate from house detection.

\subsubsection{Road Detection}

There is an excellent survey paper by Mayer et al. [218] on road detection in aerial images. As in the previous section, we add unreported papers in this survey following the same format (simplified) in Table 7.3. For each paper, we provide (if available) the data complexity, representation, and the resolution in meters per pixel.

\begin{tabular}{|l|c|c|c|}
\hline Approach & Complexity & Representation & Resolution \\
\hline Bajcsy and Takavoli [219] & low & topology & $79 \mathrm{~m}$ \\
\hline Wang and Newkirk [220] & medium & semantic network & $10 \mathrm{~m}$ \\
\hline Fiset and Cavayas [221] & medium-high & topology, tracking & $30 \mathrm{~m}$ \\
\hline Netanyahu et al. $[222]$ & medium & parametric & $1-3 \mathrm{~m}$ \\
\hline Tupin et al. $[223]$ & high & semantic network & \\
\hline Karathanassi et al. $[224]$ & medium-high & topology, parametric & $6.25,10 \mathrm{~m}$ \\
\hline Laptev et al. $[225]$ & low & snakes & $0.5 \mathrm{~m}$ \\
\hline Jeon and Hong [226] & low & grouping, snakes & \\
\hline Shi and Zhu [227] & high & topology & $1 \mathrm{~m}$ \\
\hline
\end{tabular}

Table 7.3: Road detection review

As Table 7.3 shows, images with various complexities are used to detect road networks. For road detection, airborne, satellite, and SAR images are used. Topology, parametric models, snakes, and semantic networks are the most popular representation methods. Resolution varies from 0.5 meters to 75 meters for these studies. In the low resolution (higher than $30 \mathrm{~m} /$ pixel) images only highways are detected. 
In their survey, Mayer et al. did not provide road detection performances. As in building detection, we provide the reported road detection performances in terms of probability of detection $P_{d}$ and probability of false alarm $P_{f}$ in Table 7.4.

\begin{tabular}{|l|r|r|}
\hline Approach & $\mathbf{P}_{\mathbf{d}}(\%)$ & $\mathbf{P}_{\mathbf{f}}(\%)$ \\
\hline Bajcsy and Takavoli [219] & $85.0-100.0$ & \\
\hline Wang and Newkirk [220] & 87.7 & 10.6 \\
\hline Fiset and Cavayas [221] & $79.3-81.3$ & \\
\hline Karathanassi et al. $[224]$ & 92.0 & \\
\hline Laptev et al. $[225]$ & $72.0-84.0$ & $1.0-5.0$ \\
\hline Jeon and Hong [226] & 92.2 & 1.6 \\
\hline Shi and Zhu [227] & $91.5-92.1$ & 1.6 \\
\hline
\end{tabular}

Table 7.4: Road detection review, performances

$P_{d}$ varies from $72.0 \%$ to $100.0 \% ; P_{f}$ varies from $1.0 \%$ to $10.6 \%$. In these studies, the performance depends on the type of the road to be detected, whether it is a highway with six lanes, a street in a city, or a road in a rural region. A method designed for a specific road type may not be useful for the remaining road types. Based on these, Table 7.4 gives a general idea about performance, not a conclusive test to pick the best method among them.

\section{Combined Building and Road Detection}

Besides detecting buildings and road networks separately, some researchers have designed systems to detect both simultaneously. Because buildings and road networks are not independent of each other, this strategy may improve the detection of both. Nagao et al. [228] and Nagao and Matsuyama [229] introduced such a system. Similarly, Hwang et al. [230] introduced a hypothesis generation and testing method 
to detect houses and road segments. In this chapter, we also detect houses and street networks simultaneously.

\subsection{Using Multispectral Information}

Most prior work on house and street detection uses either grayscale images or DSM data. Besides grayscale images, we have additional multispectral information. We use this information to infer cultural activity (such as houses and street networks) and water (such as lakes).

\subsubsection{A Derived Index to Detect Human Activity}

We can use vegetation indices to detect human activity in multispectral images.

We reviewed most promising vegetation indices in chapter 4 . We give the linearized version of the normalized difference vegetation index $(\theta)$ in Eqn. 4.18. The NDVI (and its linearized version $\theta$ ) responds low (around 0) to rocks, stones, and their derivatives [177]. These materials are used extensively for building and street construction, therefore indicate possible human activity (besides actual rocks in residential regions, which are rare) in residential regions. Also $\theta$ achieves high values in areas of significant vegetation density and negative values for cloud, shadow, and snow. Therefore, to measure possible building or street pixels in residential regions, we use the following derived index:

$$
\Omega=1-|\theta|
$$


$\Omega$ is normalized between 0 (suggesting low human activity) and 1 (usually meaning high human activity). Actual rocks having high $\Omega$ values, even if they are rare in residential regions, are easily eliminated in subsequent processing.

To illustrate $\Omega$, we use a part of the New Mexico image given in Fig. 7.1 (a). This neighborhood shows characteristics typical of a mature residential region. Each house has its own garden; they are well-spaced; and there are mature trees nearby. If we consider Fig. 7.1 (a) we can appreciate the challenging nature of the house and street detection problem in residential regions. Even human observers find it difficult to decide whether there are houses in some parts of the image without using context, such as partial knowledge of the street layout, driveways, and so on. We give the corresponding color coded (blue corresponds to the lowest and red corresponds to the highest values) $\Omega$ image in Fig. 7.1 (b). As expected, houses and the street network are represented by high index values (in red) in this image.

\subsubsection{Using a Shadow-Water Index to Eliminate Lakes}

As we label possible house and street network pixels with high index values by $\Omega$ image, we also label water regions (such as lakes) as such. Unfortunately, lakes and ponds often appear in residential regions. To eliminate these regions before further analysis, we introduce a method to detect water regions in this section.

In section 4.3, we introduced a set of shadow-water indices to detect shadows and water regions (such as lakes) in multispectral images. We pick the best performing shadow-water index $\gamma_{2}$ given in Eqn. 4.34.

Because the index is unable to discriminate water from shadows since both have similar responses, we use $\Omega$ and $\gamma$ to extract water regions only. To do so, we first 


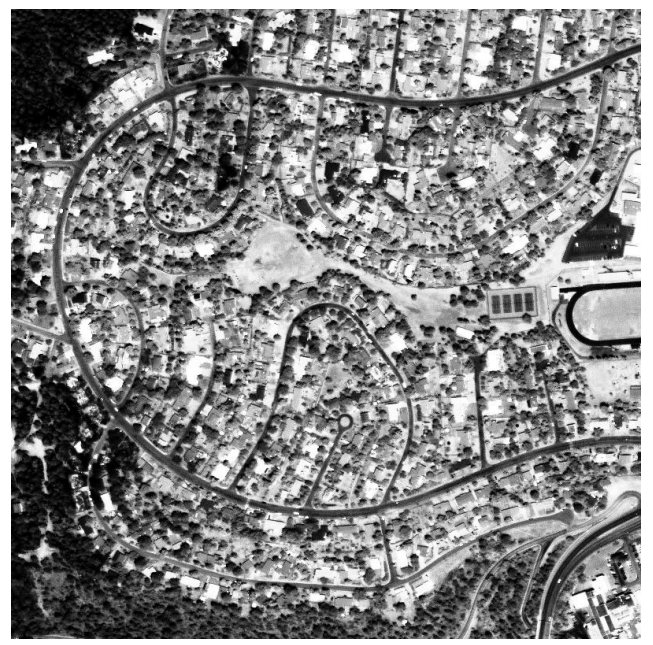

(a) The New Mexico image

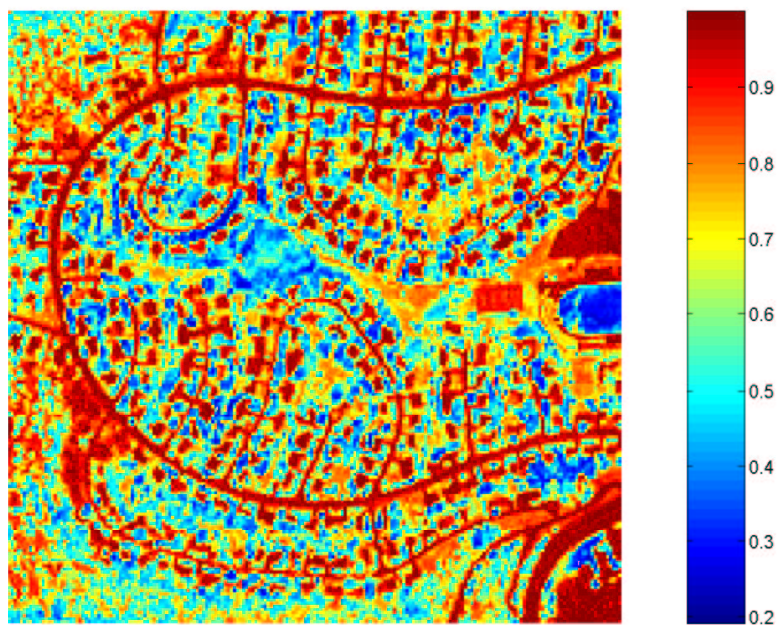

(b) $\Omega$ image (New Mexico)

Figure 7.1: $\Omega$ example

obtain a binary image from the $\gamma$ image. We experimentally observed that pixels having $\gamma$ values higher than 0.3 are possible shadows or water regions. Thus, we threshold the $\gamma$ image at this value. We then use connected components analysis to extract eight-connected regions in this binary image. We eliminate regions smaller than 75 pixels, considering them to be insignificant (neither a significant water nor a shadow region that will impact subsequent processing).

To label each eight connected region, we calculate the median values of $\theta$ and $\Omega$ over it. Since shadows lie over a region (for our case, regions are restricted to be residential) it is highly possible that the underlying region is vegetated. For water regions, although there may be some greenness in the water, its effect is minor. A water region responds high in the $\gamma$ image. Based on these observations we take a region as water, if $\theta$ median is less than 0.2 and $\gamma$ median is greater than 0.3 . 
Water detection was applied to the 44 images at hand and it worked as desired. To demonstrate this strategy, we show it on an Indiana image, containing a lake in the middle of the scene. We give its panchromatic (grayscale) image, color coded (blue corresponds to the lowest value, red corresponds to the highest value) $\gamma$ image, and the water region detected from it in Fig. 7.2. The lake is correctly detected in the middle of a residential region.

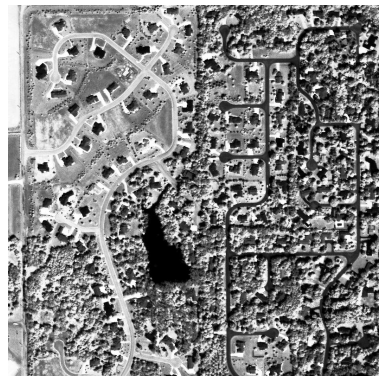

(a) The Indiana image

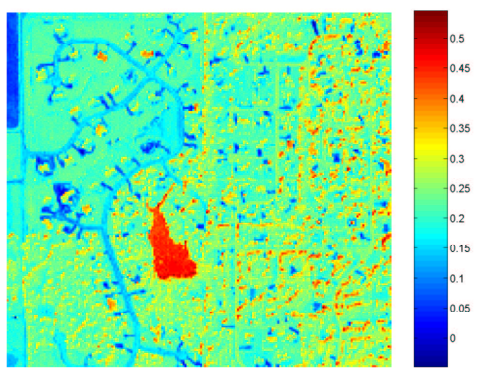

(b) $\gamma_{2}$ image (Indiana)

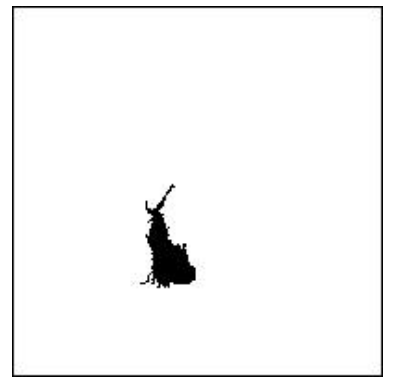

(c) The lake detected

Figure 7.2: Detecting water regions

\subsection{Segmenting the $\Omega$ Image}

Although $\Omega$ indicates possible human activity, we need a binary image for subsequent processing. In this binary image, possible human activity will be labelled as an object and the remainder as background. Therefore, we focus on segmentation algorithms in this section. We introduce an extension of the k-means clustering algorithm for segmentation. The novelty of our clustering algorithm is its exploitation of spatial coherence via connected components. 
We first explain our segmentation algorithm in the next section. Then, we compare our algorithm with the existing segmentation algorithms such as standard k-means clustering, Otsu's method, and region growing. Finally, we explore its robustness.

\subsubsection{K-means Clustering with Spatial Coherence}

K-means clustering is a standard method in pattern recognition to extract natural clusters in the data iteratively $[102,231]$. We give the k-means clustering algorithm Alg. 1 from Duda et al. [102] first. In this version of k-means clustering, the Euclidean distance is used in the classification step. Jain et al. [232] suggested the use of the Mahalanobis distance, instead. No spatial information is used in either case.

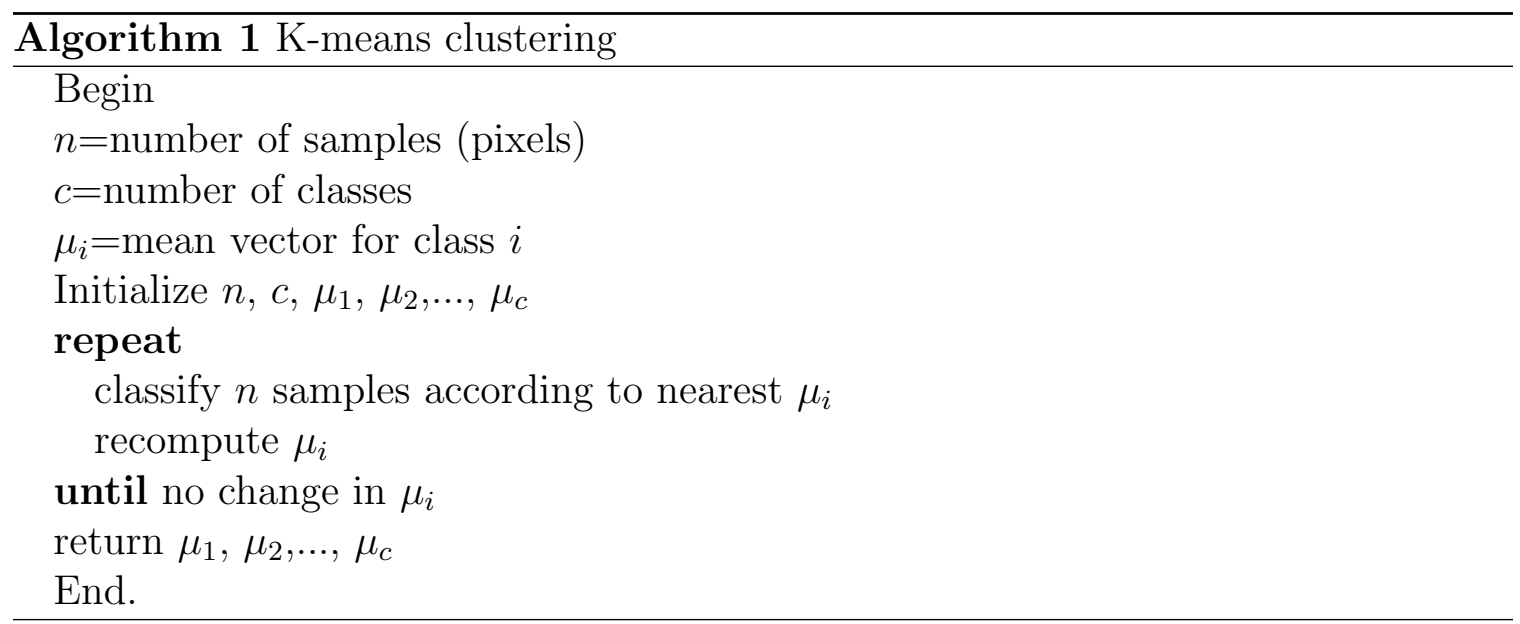

As we observe the $\Omega$ image in Fig. 7.1, houses and street segments are connected via driveways. The street network is also connected. To use this information, we extend the k-means clustering algorithm by introducing the spatial information via connected components. We call our algorithm k-means clustering with spatial coherence. 
Our application of k-means clustering with spatial coherence is as follows. We first obtain the $\Omega$ image from the red and near-infrared bands as given in Eqn. 7.1. On this image, we define an initial segmentation threshold $t_{s}$. The pixels with $\Omega$ values greater than $t_{s}$ are the initial object pixels (possible human activity), and the rest are the initial background pixels. These serve as input to the class-conditional sample mean and covariance calculations. K means clustering with the Mahalanobis distance is then applied to the data to obtain new object and background pixels. Here, the use of spatial coherence begins. The new object pixels are decomposed into eight-connected sets. Those sets intersecting with the initial object form the final object segment. Therefore, the object class is kept coherent throughout clustering. The initial object class is updated accordingly. A new iteration starts by updating the sample mean and covariance calculations. The iteration terminates when the absolute value of the difference between the current and previous object (total) pixel counts falls below a threshold. The detailed description is given in Alg. 2 .

We demonstrate the algorithm on four images from different regions. For each case, we give the panchromatic image and the binary segment $\left(C^{o}\right)$ in Figs. 7.3 and 7.4. On all test images, our clustering method was able to converge in a reasonable number of iterations (approximately 10 to 12 iterations on the average). These experiments show that our clustering method works fairly well on different residential regions and varying environmental conditions including changes in vegetation cover and soil type.

\subsubsection{Comparison with Other Methods}

We will now compare our modified clustering method with standard k-means clustering, Otsu's method [233], and region growing [234,235]. We also tested fuzzy 


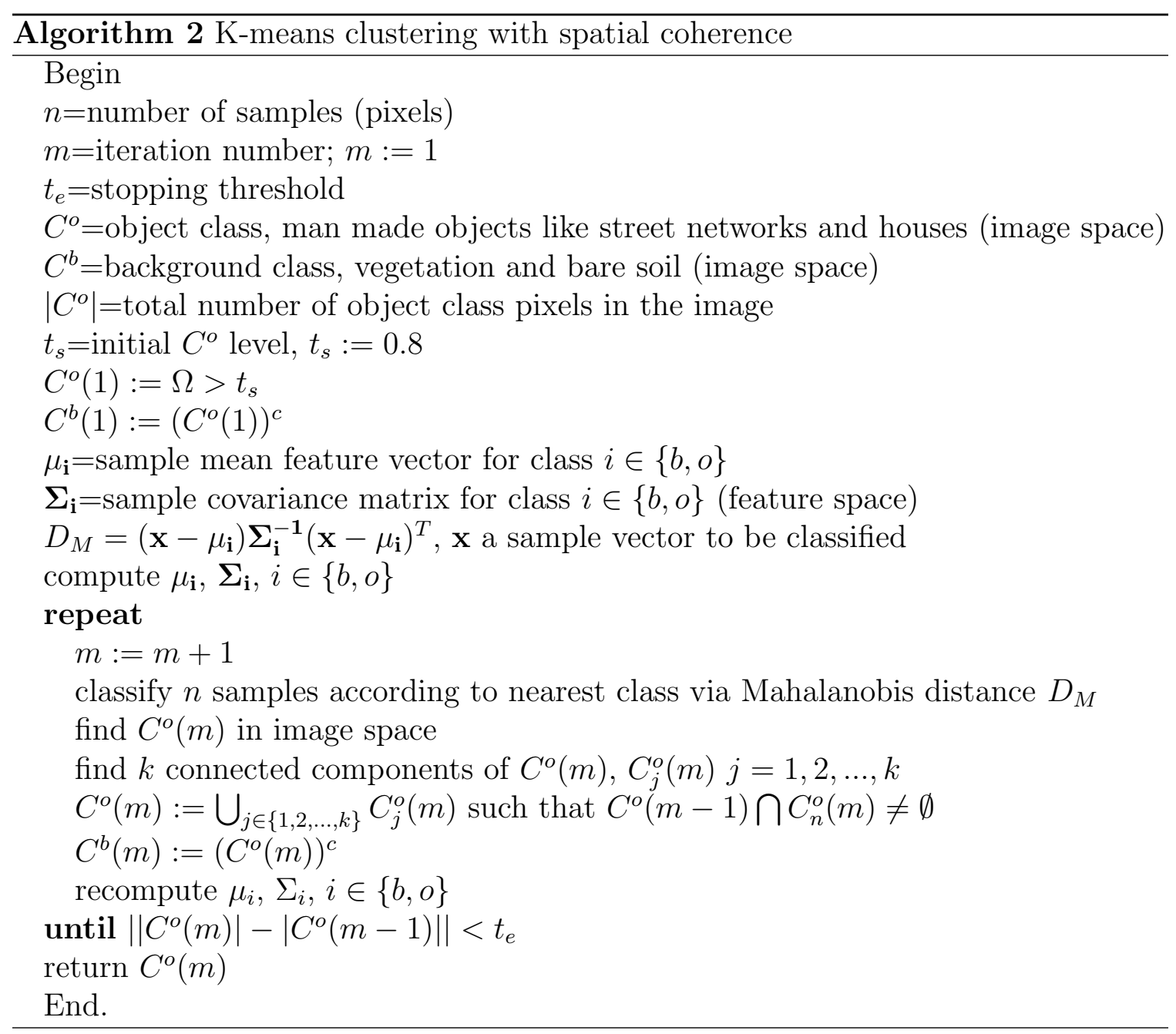




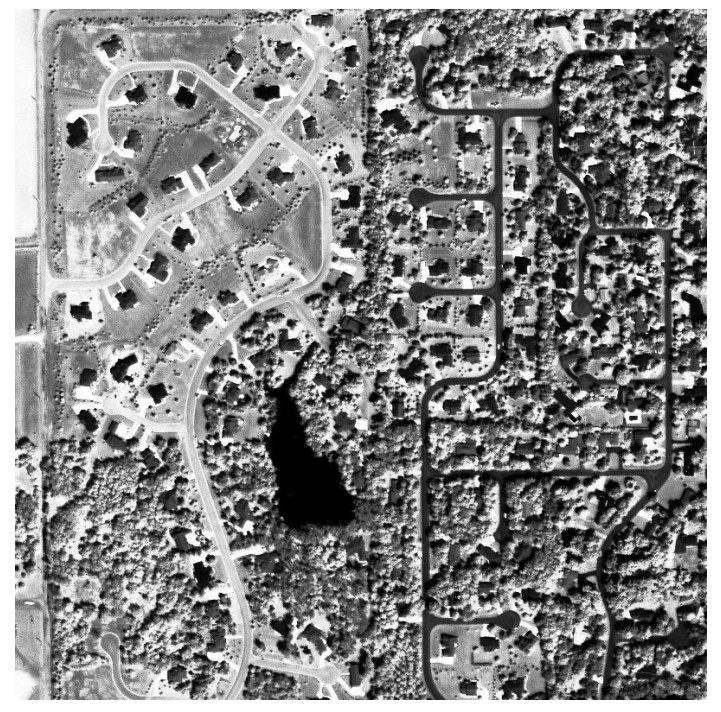

(a) The Indiana panchromatic image

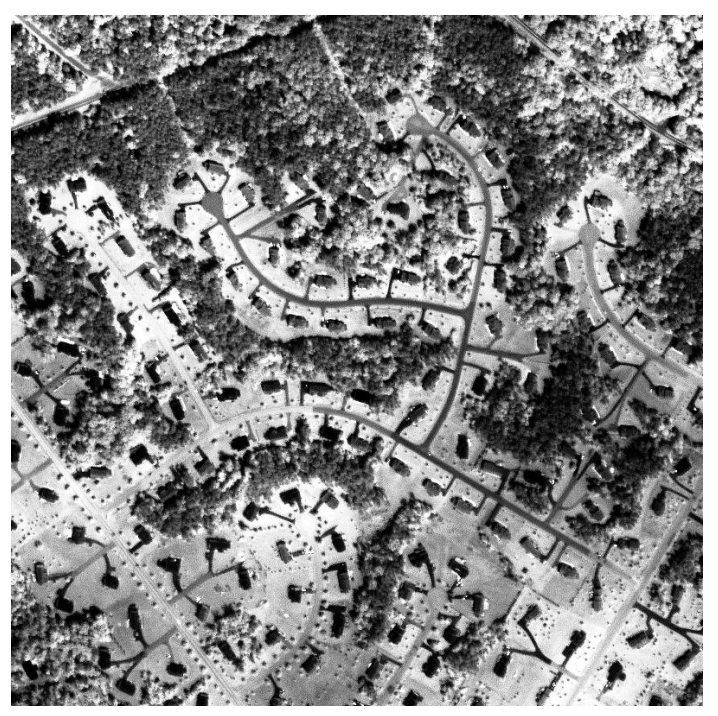

(c) The Maryland panchromatic image

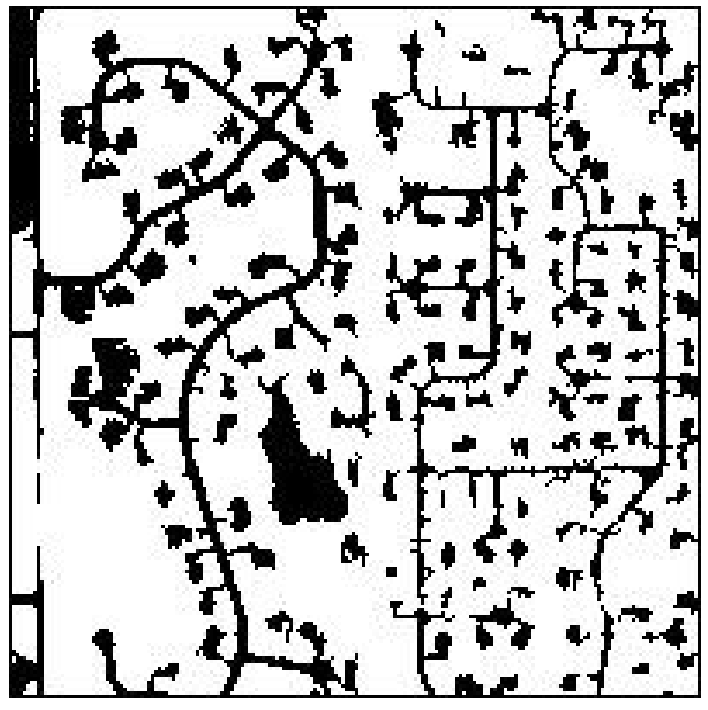

(b) The binary segment $\left(C^{o}\right)$

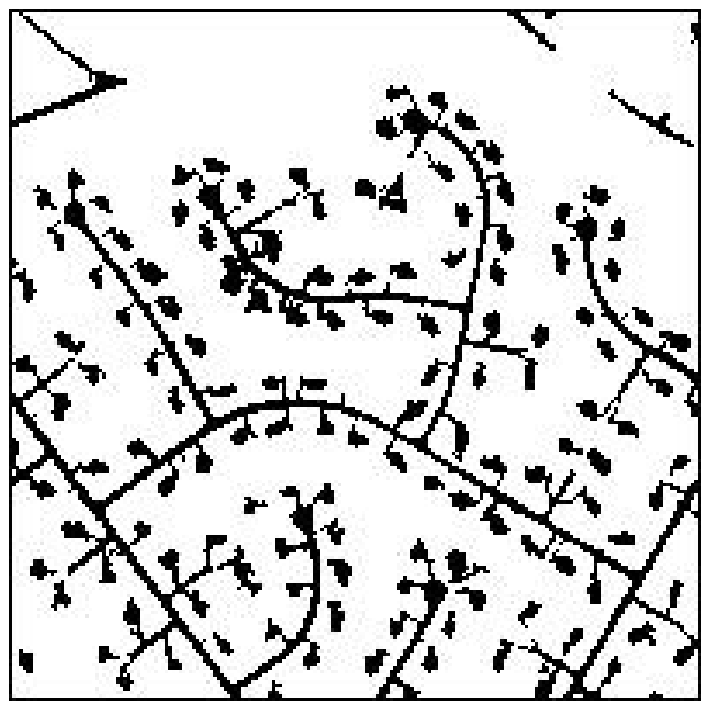

(d) The binary segment $\left(C^{o}\right)$

Figure 7.3: Test images and binary segments $C^{o}$ returned by Algorithm 2 - Part I 


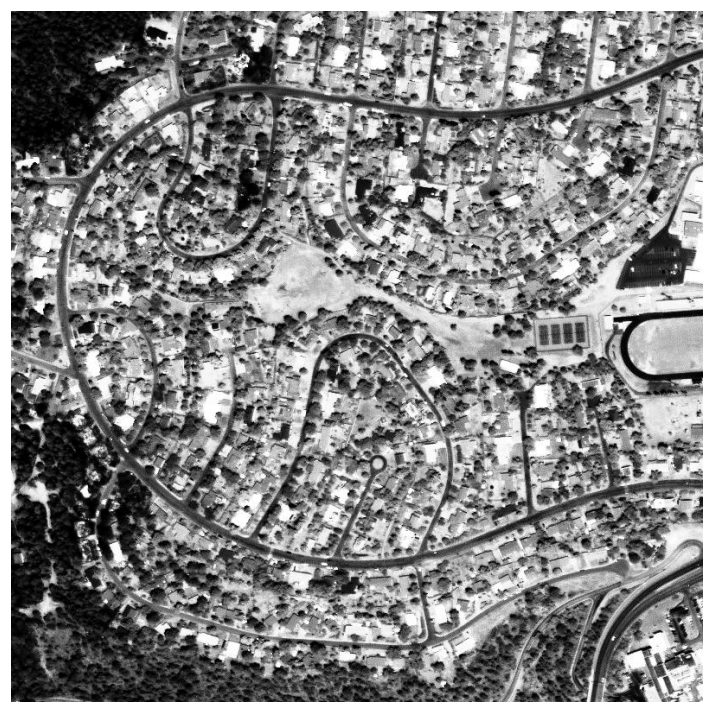

(a) The New Mexico panchromatic image

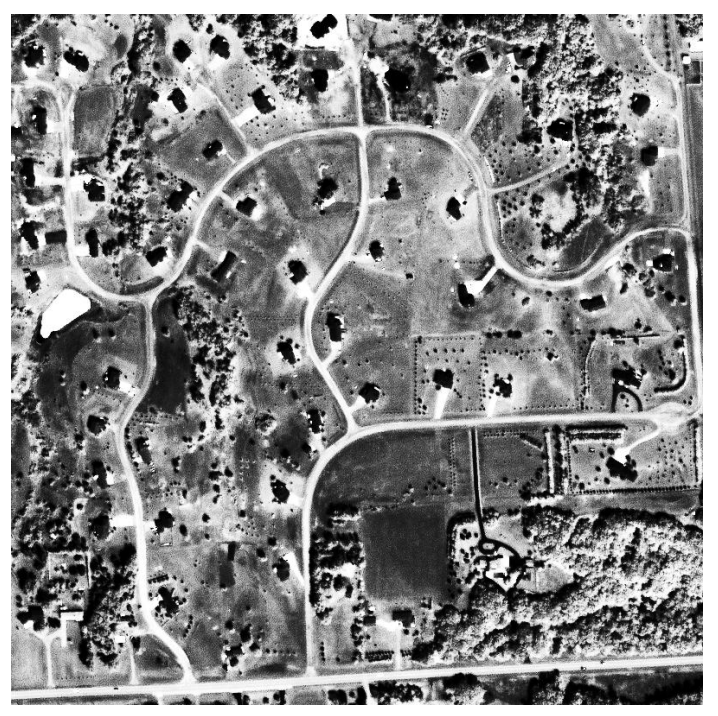

(c) The South Dakota panchromatic image

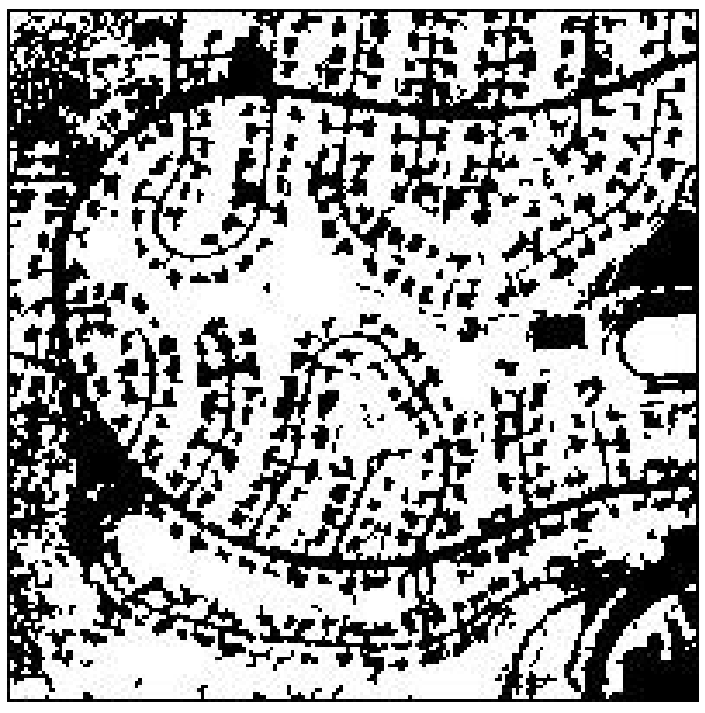

(b) The binary segment $\left(C^{o}\right)$

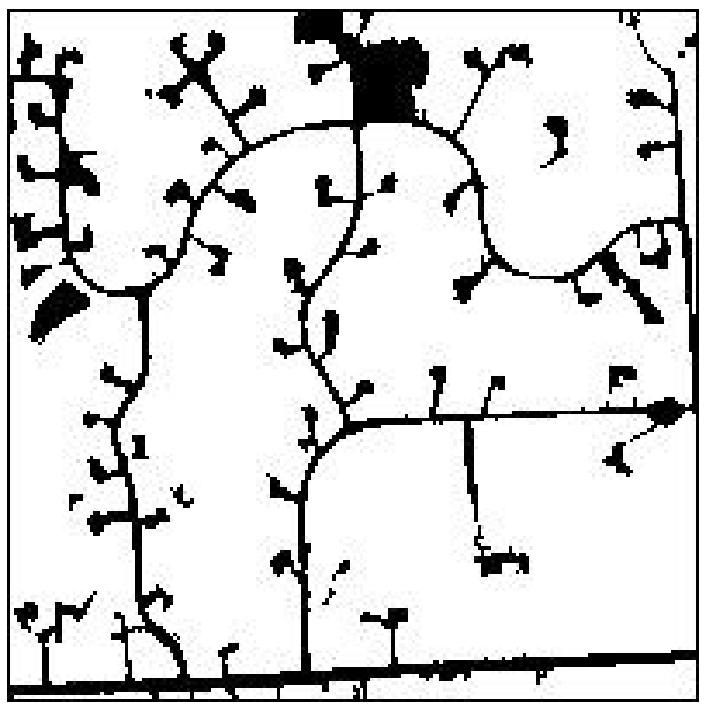

(d) The binary segment $\left(C^{o}\right)$

Figure 7.4: Test images and binary segments $C^{o}$ returned by Algorithm 2 - Part II 
k-means clustering [102] but the performance was similar to k-means clustering, therefore we omit it here.

Otsu obtains a threshold for object-ground separation by defining an optimality condition. He formulates the optimum threshold value by maximizing the betweenclass variance assuming a bimodal histogram such that one peak corresponds to the object and the other to the background. This method depends on the histogram only. Although it is extremely fast, if the bimodal histogram assumption is not satisfied, the results become less reliable.

In region growing, initial seed points are hand labelled or selected by some other process. Then, nearby points are added to these seed points such that they do not exceed a threshold in variance or another homogeneity constraint. Here, we obtained the seed points by thresholding, and for homogeneity we used the variance threshold. One shortcoming of region growing is its heavy dependence on seed points. Also, as the region area grows, the incursion of false pixels to clusters becomes more probable [235].

We compare these four methods in Fig. 4.12. Here, we use four different residential regions: Indiana, Maryland, New Mexico, and South Dakota. Segmentation by standard k-means clustering (KMC) performed worst of all. We see the effects of heavy dependence on the seed points in region growing on the Indiana and Maryland images. The method can not recover regions having no seed points. Finally, k-means clustering with spatial coherence performed well on all four images. We also quantify these results (in percentages) in Table 7.5. 

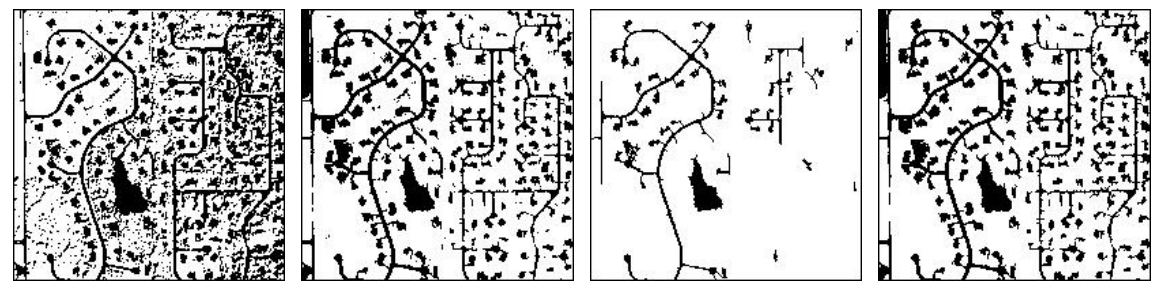
(a) $\mathrm{KMC}$
(b) Otsu's
method
(c) Region
(d) KMC
with spatial
coherence
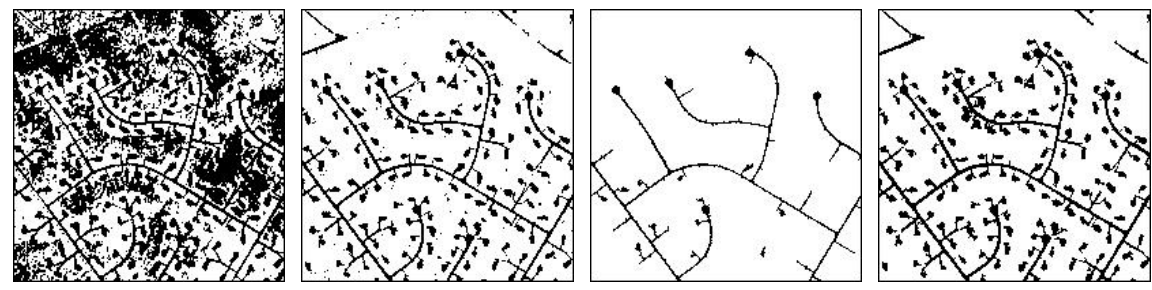

(e) KMC

(f) Otsu's
method

(g) Region
growing

(h) KMC

with spatial

coherence
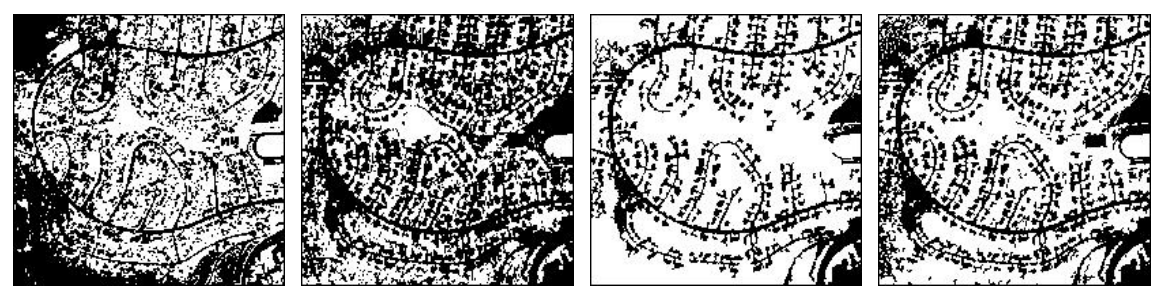

(i) $\mathrm{KMC}$

(j) Otsu's method

(k) Region

(1) KMC with growing spatial coherence
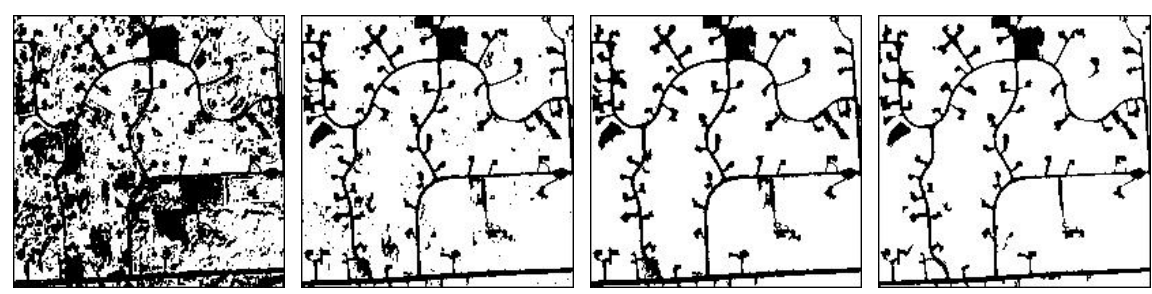

(m) KMC

(n) Otsu's method (o) Region growing (p) KMC with spatial coherence

Figure 7.5: Comparison of segmentation algorithms 


\begin{tabular}{|l||c|c||c|c||c|c||c|c|}
\hline \multirow{2}{*}{} & \multicolumn{2}{|c|}{ Indiana } & \multicolumn{2}{c|}{ Maryland } & \multicolumn{2}{c|}{ New Mexico } & \multicolumn{2}{c|}{ S. Dakota } \\
\cline { 2 - 9 } & $P_{d}$ & $P_{f}$ & $P_{d}$ & $P_{f}$ & $P_{d}$ & $P_{f}$ & $P_{d}$ & $P_{f}$ \\
\hline KMC & 78.0 & 49.0 & 86.3 & 38.7 & 20.2 & 56.9 & 96.2 & 34.1 \\
\hline Otsu's method & 91.9 & 6.4 & 85.3 & 1.6 & $\mathbf{9 6 . 7}$ & 36.6 & 98.2 & 7.9 \\
\hline Region growing & 26.8 & $\mathbf{2 . 1}$ & 25.4 & $\mathbf{0 . 2}$ & 74.0 & $\mathbf{6 . 7}$ & 96.9 & 6.6 \\
\hline KMC with spatial coherence & $\mathbf{9 4 . 4}$ & 5.6 & $\mathbf{9 6 . 4}$ & 1.0 & 95.3 & 13.2 & $\mathbf{9 8 . 7}$ & $\mathbf{3 . 3}$ \\
\hline
\end{tabular}

Table 7.5: Comparison of performances

As Table 7.5 shows, KMC with spatial coherence has the highest $P_{d}$ over three images. It falls behind Otsu's method only in the New Mexico image. Region growing has the lowest $P_{f}$ for three images except South Dakota. However, for these three images, it has $P_{d}$ values far below KMC with spatial coherence. Overall, KMC with spatial coherence is the best segmentation method among these four for our application.

\subsubsection{Dependence on Initial Conditions}

The only free parameter in the KMC with spatial coherence algorithm is the initial segmentation threshold $t_{s}$. This also serves as the seed point extraction step in region growing. Here, we test the stability of our method with respect to this initial threshold value. We consider three different initial segmentation thresholds ranging from $t_{s}$ very large to very small. We give the initial and the final segments in Fig. 7.6. We also provide the total pixel count in the segment versus the iteration number, showing that convergence is nearly exponential.

As can be seen, the selection of $t_{s}$ is not highly critical. If the segmentation threshold is high (fewer seed points), the method is able to recover most of the missing parts. If the segmentation threshold is low (seed points cover more area than 


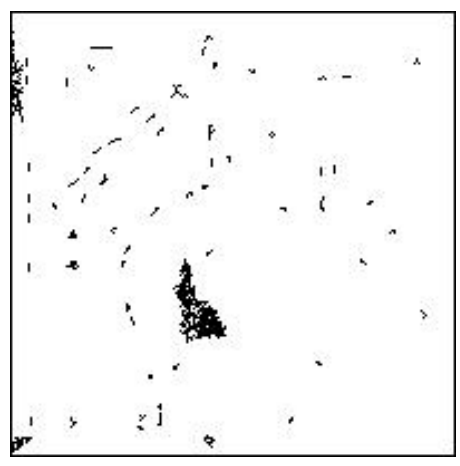

(a) The initial segment, $t_{s}=0.95$

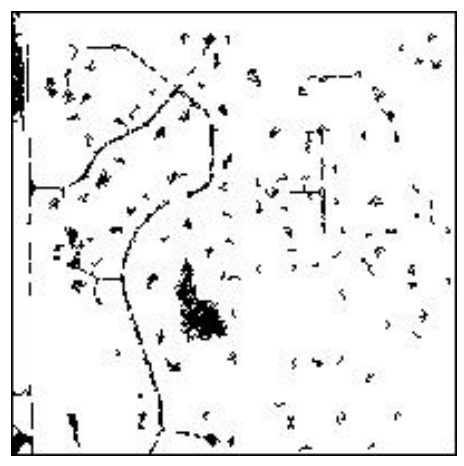

(d) The initial segment, $t_{s}=0.80$

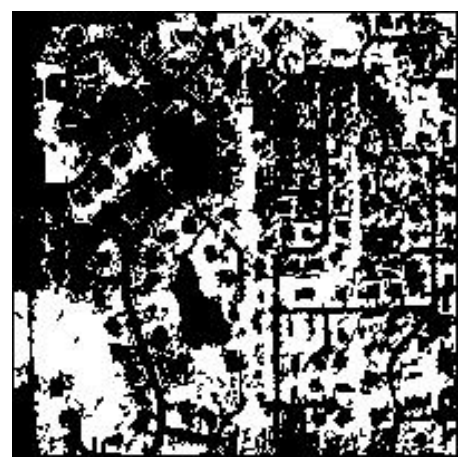

(g) The initial segment, $t_{s}=0.40$

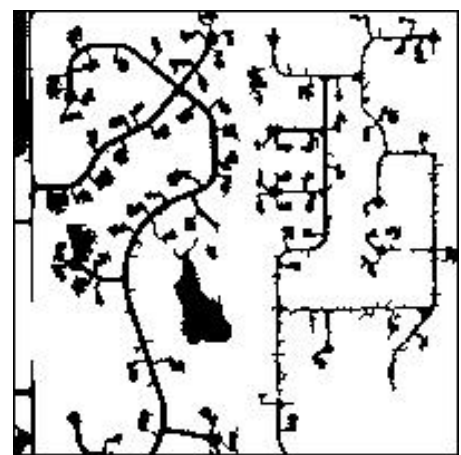

(b) The final segment $\left(C^{o}\right)$

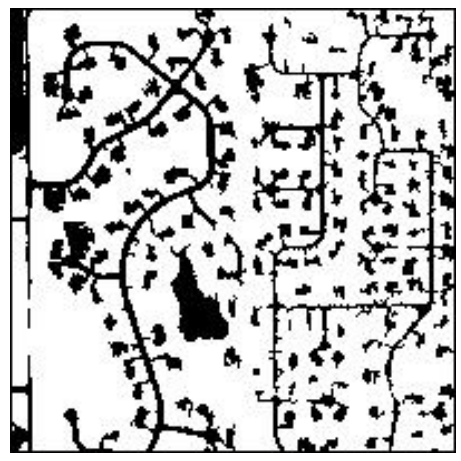

(e) The final segment $\left(C^{o}\right)$

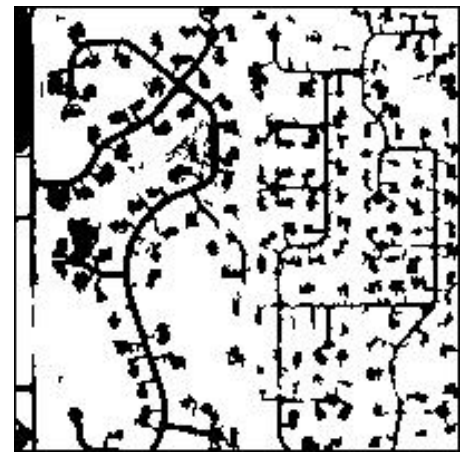

(h) The final segment $\left(C^{o}\right)$

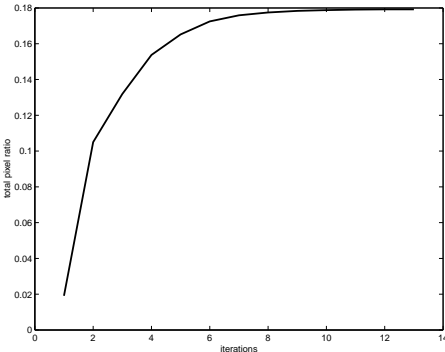

(c) \# pixels vs. iteration

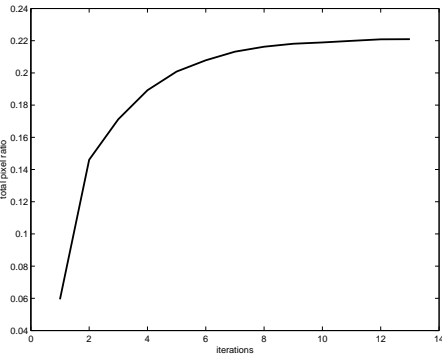

(f) \# pixels vs. iteration

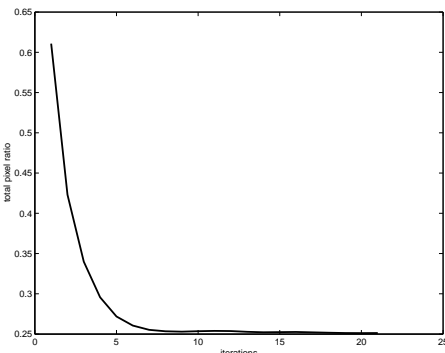

(i) \# pixels vs. iteration

Figure 7.6: The Indiana image, segmentation results with different $t_{s}$ values 
the actual shape), the method is able to eliminate most extra parts. Of course, if a reasonable threshold is selected we get the best performance. The threshold can also be calculated by another algorithm (such as Otsu's method), however we found no significant gain in doing so. Since the $\Omega$ image is scaled, a constant threshold for all images suffices.

\subsection{Shape Decomposition of the Foreground: The Binary Balloon Algorithm}

We next decompose the binary segment $C^{o}$ (representing possible houses and the street network) into subsets such that each either represents a structure (a house or a street segment), or a combination represents such a structure. From this point on, $C^{o}$ represents a binary segment without lakes and water regions. We discussed how to discard these regions in Section 7.2.2.

For decomposition, we introduce a binary balloon algorithm. Balloons are deformable models that are fitted to a target object in an image using external and internal constraints [236]. External constraints are provided by image (photometric) gradients, while the internal constraints are smoothness and bending forces designed to limit the balloon's shape complexity. Balloon algorithms have been used extensively for shape fitting in computer vision.

The extent of the objects in $C^{o}$ motivates us to introduce a new balloon algorithm tailored to our domain. We construct the binary balloons by set morphology operations. Mathematical morphology is a well-known tool to extract regions based on their shapes $[235,237]$. We now provide a very brief review of mathematical morphology basics. Mathematical morphology (more properly, Minkowski algebra) is based on logical operations over, in our case, sets in $Z^{2}$. We need three basic morphology set 
operations: translation, dilation, and erosion. We consider each in turn. Let points (vectors) be $\mathbf{p}=\left(p_{1}, p_{2}\right), \mathbf{a}=\left(a_{1}, a_{2}\right), \mathbf{b}=\left(b_{1}, b_{2}\right)$. Let $A$ be a set of points, not necessarily connected, in the plane.

The translation of set $A$ by $\mathbf{p}$ is:

$$
(A)_{p}=\{c \mid c=a+p, a \in A\}
$$

The dilation of set $A$ by set $B$ is:

$$
A \oplus B=\bigcup_{b \in B}(A)_{b}
$$

The erosion of set $A$ by set $B$ is:

$$
A \ominus B=\bigcup_{b \in B}(A)_{-b}
$$

Set $B$ as used in Eqs. 7.3, 7.4 is commonly referred to as the "structuring element". It is a sort of template for the analysis of the shape of set $A$.

\subsubsection{Constructing Initial Balloons}

Our primary goal in decomposing the binary image $C^{o}$ is to identify its elongated subsets such that they represent possible street segments (non-elongated segments will represent other structures, including houses). For this purpose, we use lines as initial balloons in $C^{o}$. We extract lines by applying the erosion and dilation operations, respectively. We define four linear structuring elements (one could use more, but we found no advantage in doing so): horizontal, vertical, diagonal-1, and diagonal-2. If we take $x$ and $y$ as coordinates in the image space, horizontal corresponds to a constant $y$, vertical corresponds to a constant $x$, diagonal-1 corresponds to a line 
segment $y=x+b, b$ being the y-intercept, and diagonal-2 corresponds to a line segment $y=-x+c, c$ being the y-intercept.

To extract initial balloons for each structuring element, we apply Alg. 3. The procedure is briefly as follows. We set the length of the structuring element to the maximum image size to detect the longest initial balloon possible. We then decrease the length of the structuring element until we detect an instance of it in the image. This structuring element is taken as an initial balloon. Then, we expand the initial balloon to construct the corresponding binary balloon and eliminate its pixels from the image via set subtraction. By decreasing the size of the structuring element, we continue to detect initial balloons and expand the corresponding binary balloons. Since we discard the points in each binary balloon before extracting the next, the final set of balloons will be disjoint.

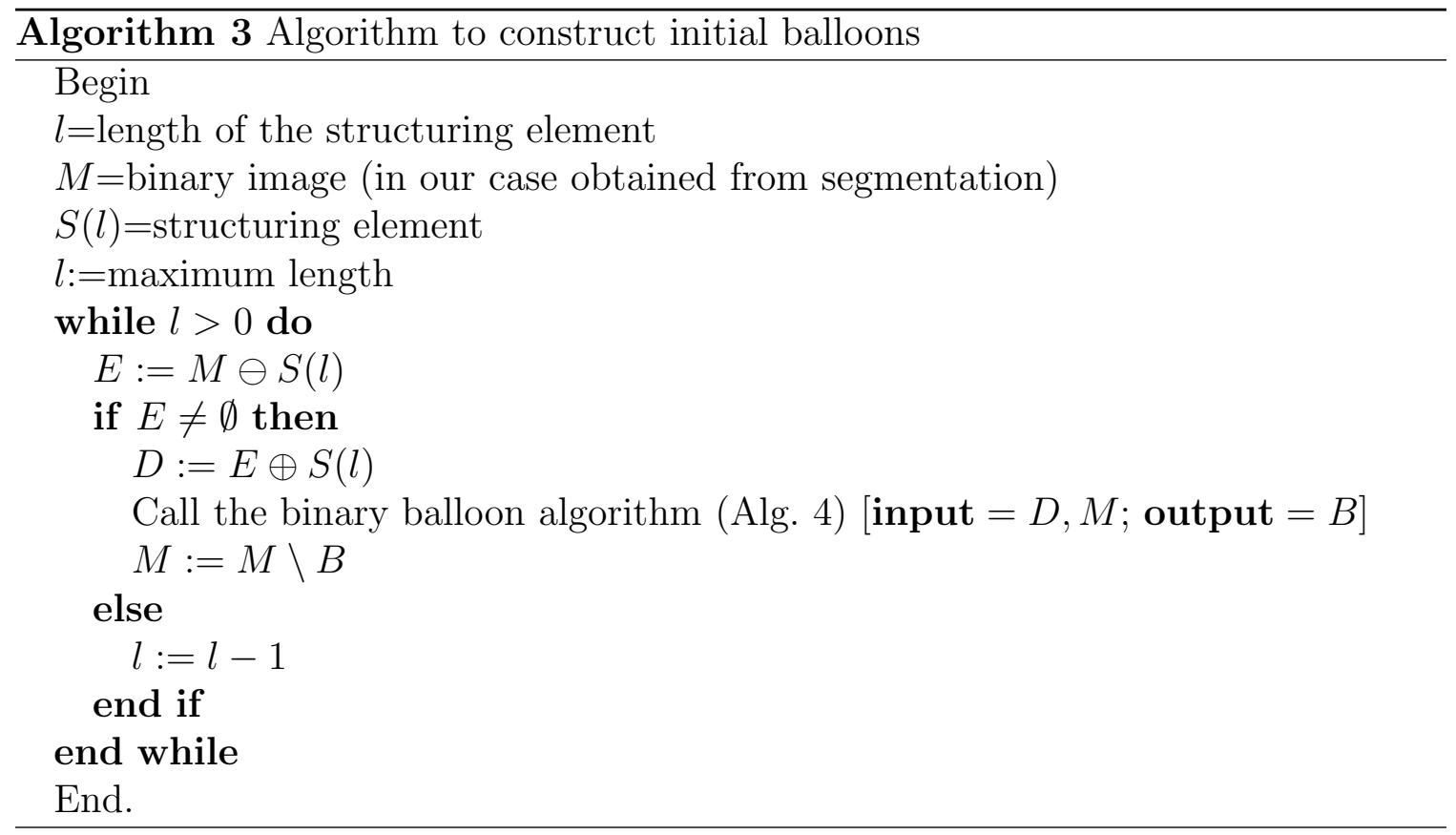




\subsubsection{Constructing the Binary Balloons}

Once we obtain an initial balloon, we next construct the corresponding binary balloon. We follow the same methodology by expanding the initial balloon to fit into a region as in standard balloon algorithms. However, unlike other algorithms, we do not use a parametric form for the balloon. Instead, we expand each balloon by adding neighboring points satisfying a smoothness condition. This is a unique feature of our binary balloon algorithm. We had to follow such a strategy because standard balloon algorithms did not perform reasonably in our domain owing to the extreme aspect ratios of streets and roads, and small house sizes.

We start with a binary balloon obtained from Alg. 3. The outer boundary points (eight-connected neighbors) of the initial balloon are labelled as candidate points. If there are no such pixels, the balloon expands no more. Therefore, our balloon can never expand beyond the object.

We group the candidate pixels into eight connected candidate sets, by connected components analysis. We want a smooth boundary for the final balloon. To ensure the smoothness of the boundary, each added candidate set should be similar to the balloon locally. This will be our internal constraint. Since all pixels in each candidate set are eight-adjacent to the current balloon, larger sets are more consistent in shape with the current balloon than are the smaller sets. Therefore, we define a size threshold $t_{o}$ to control the boundary smoothness. If we keep this threshold high, the decomposition of $C^{o}$ will consist of more balloons with smooth boundaries; if we keep it low, fewer balloons with rougher boundaries will result. If the total number of adjoining pixels exceeds $t_{o}$, we add the candidate set to the balloon. We update the balloon, and iterate until the balloon expands no more. 
Initial additions will be lines, since we start with a line. As the balloon starts to take the shape of the object, curved lines can be added. At every step, the size threshold will force the shape to remain smooth. The binary balloon construction algorithm appears in Alg. 4.

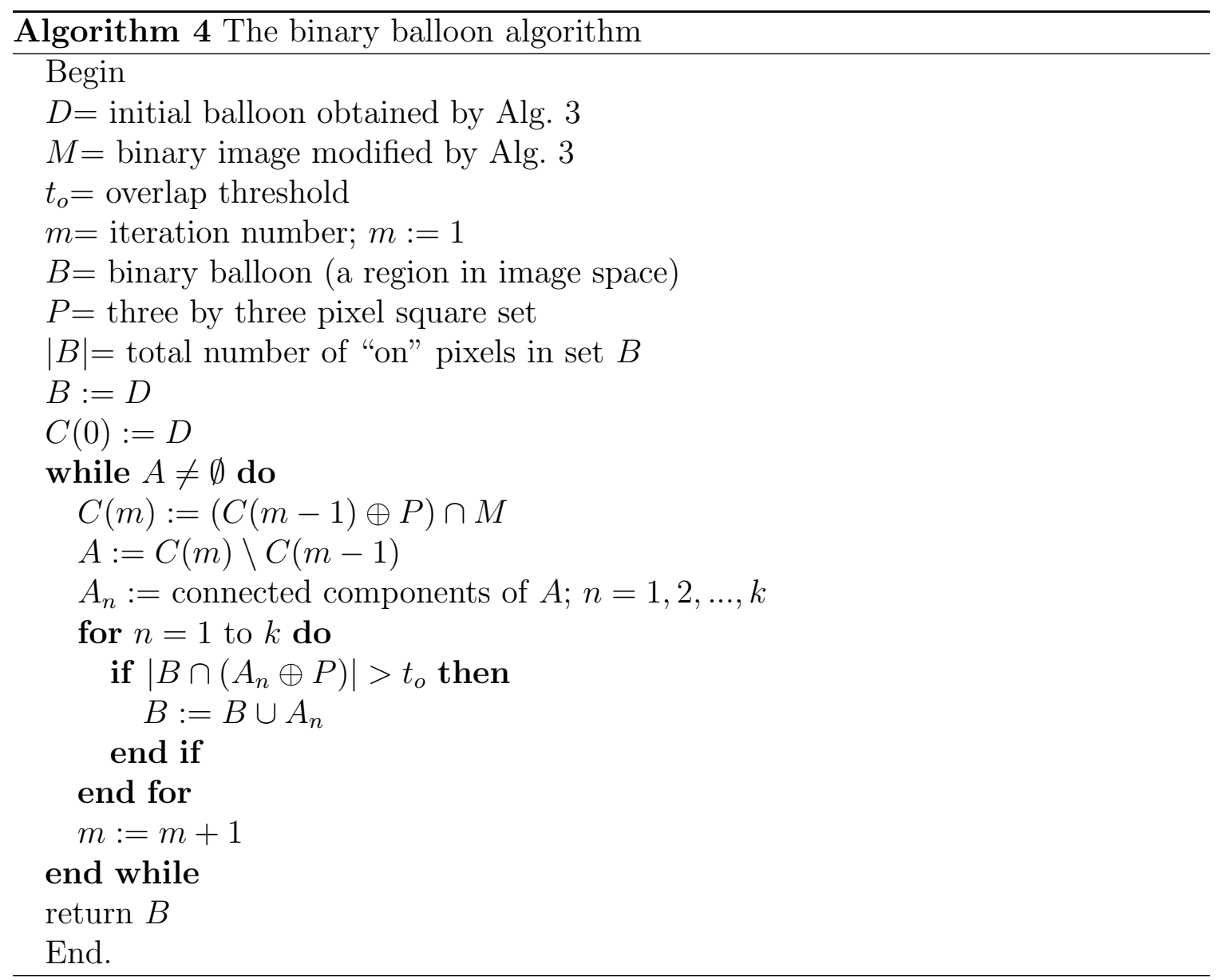

To clarify these steps, we demonstrate them on a small example in Fig. 7.7. Here, dashed blue lines represent candidate points and red points represent the extracted balloon for each iteration. We start with the initial horizontal balloon (labelled red) in Fig. 7.7 (b). Two candidate sets (labelled in green) exceed $t_{o}$ (separately) at the 
first iteration, therefore will be added to the balloon. Two candidate sets (labelled yellow) could not exceed $t_{o}$ at the second iteration. Also all the pixels on the object have been checked, hence the iteration stops. The final balloon extracted is labelled in red in Fig. 7.7 (d).

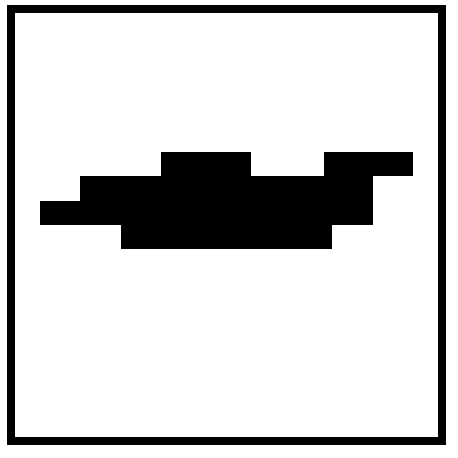

(a) The object

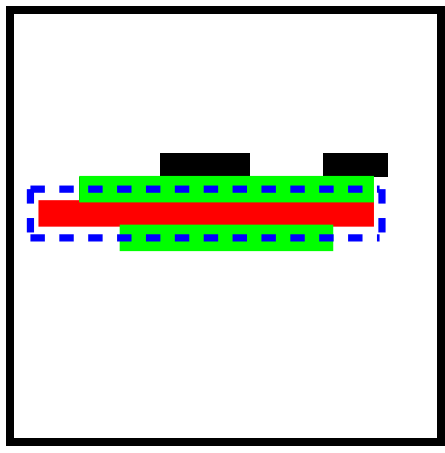

(c) The first iteration, blue- candidate points, green - accepted candidate sets

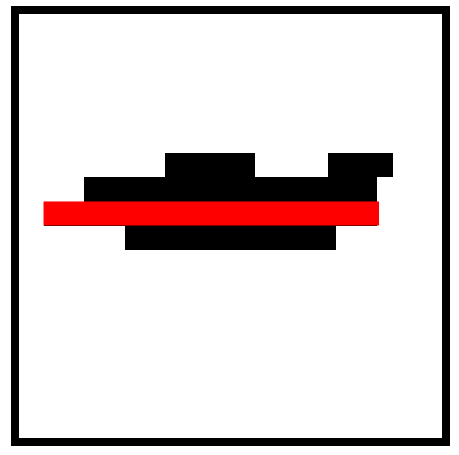

(b) The initial balloon, labelled red

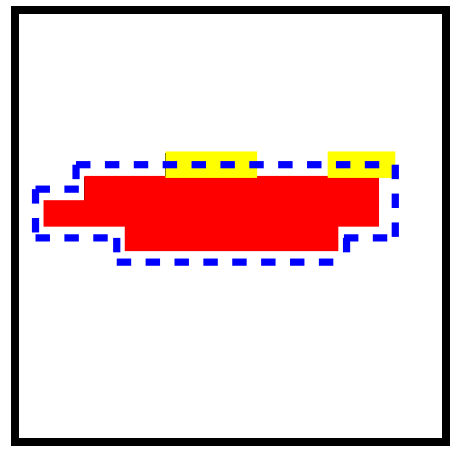

(d) The second iteration, yellow - rejected candidate sets, red - the final balloon

Figure 7.7: Extracting the binary balloon, an example 
Next, we demonstrate binary balloon extraction on the South Dakota image. To obtain the decomposition for $C^{o}$ in this image, we obtain all the binary balloons in $C^{o}$ with the four structuring elements separately as illustrated in Fig. 7.8. For demonstration purposes, we applied a greedy graph coloring algorithm in all these figures, such that no two neighboring balloons have the same color [238]. In this figure, gray levels have no meaning except as labels to distinguish one balloon from another.

In Fig. 7.8 (a), the decomposition of $C^{o}$ into horizontal balloons is shown. In this decomposition, there are around 200 horizontal non-intersecting balloons covering $C^{o}$. Horizontal $C^{o}$ sections are represented by a few large balloons; curved sections are represented by many small balloons. This is also the case for vertical and diagonal decompositions with respect to their initial balloons. All balloons have smooth boundaries because of the size threshold (internal constraint) is kept high. Most of them are also elongated. Next, we combine these four representations.

\subsubsection{Combining Balloons via Voting}

As we apply the decomposition method, each pixel in the $C^{o}$ segment belongs to at least one balloon. These multiple representations result in many overlapping and redundant balloons. To merge these balloons and eliminate redundancy as much as possible, we apply the voting method introduced by Burns et al. [96] (explained in section 3.1). In this method, each pixel votes for the largest balloon of which it is a member. As votes are summed, those balloons receiving votes exceeding $80 \%$ of their areas are selected and retained. The rest are deleted as redundant since they do not convey new information. The merging does not result in a perfectly disjoint balloon 


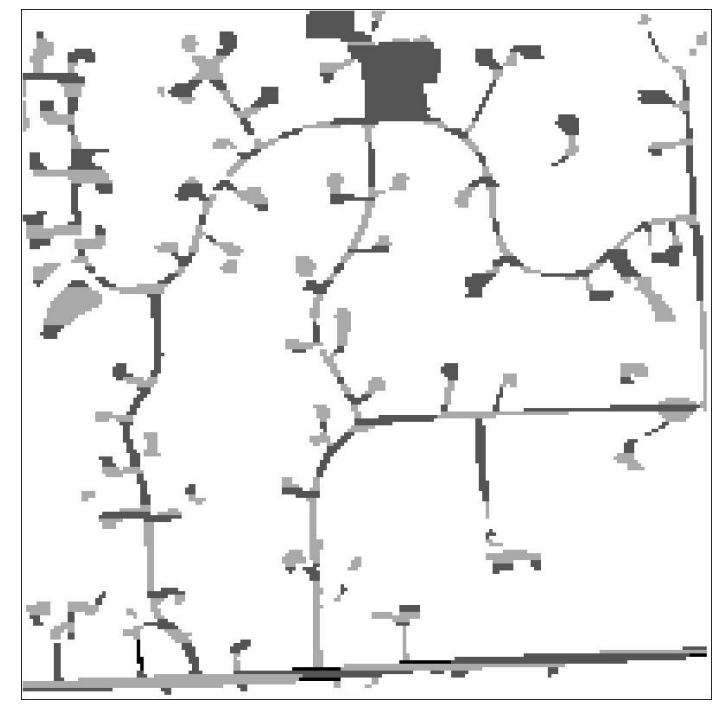

(a) Horizontal balloons

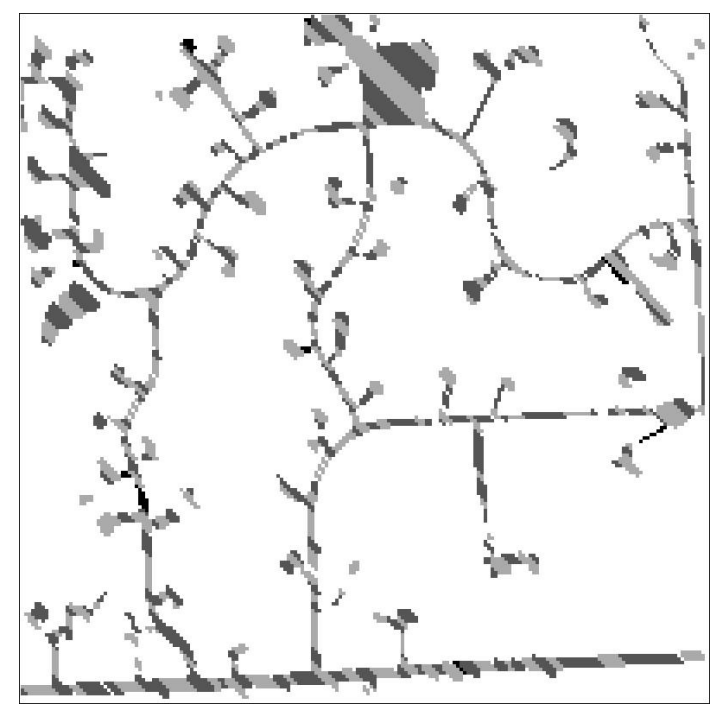

(c) Diagonal-1 balloons

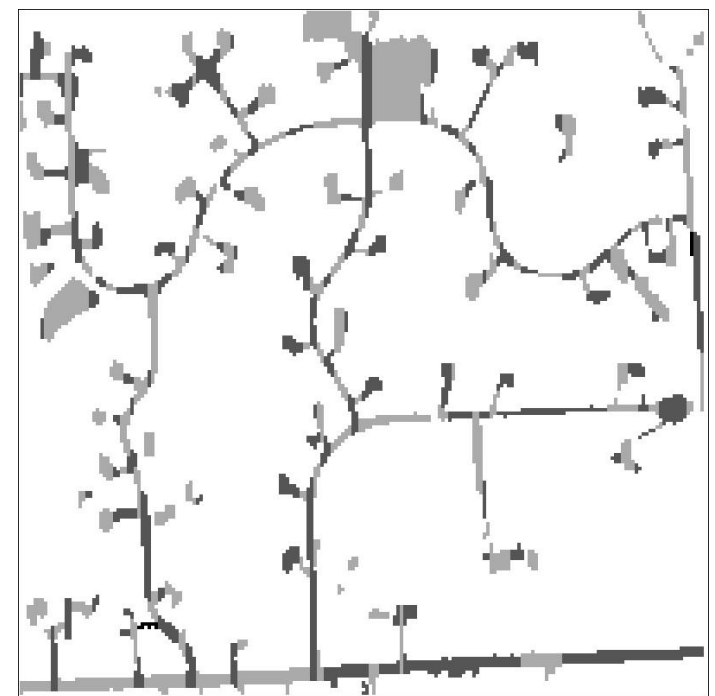

(b) Vertical balloons

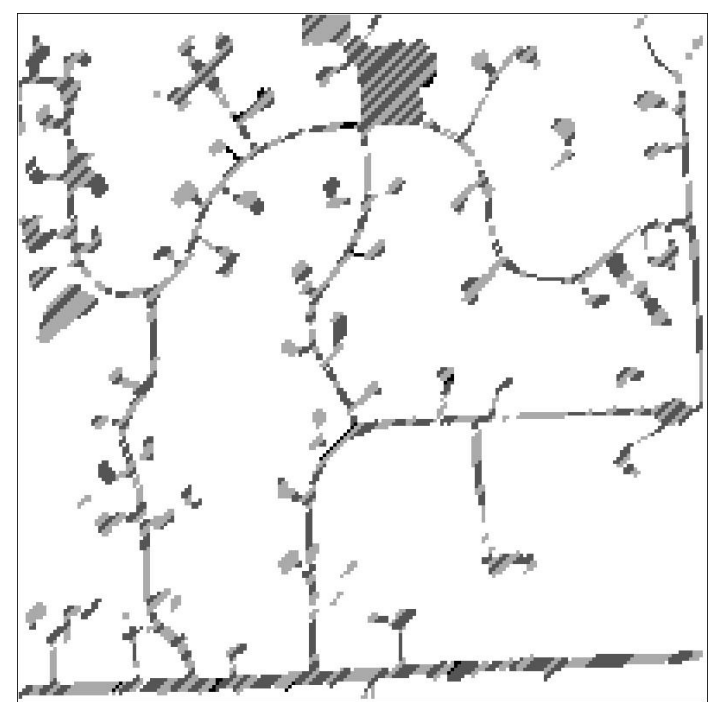

(d) Diagonal-2 balloons

Figure 7.8: The South Dakota image, decomposition of its $C^{o}$ 
set. The balloons may overlap somewhat around connecting regions; however, this overlap does not impair subsequent processing.

Applying the combination method to the balloons in Fig. 7.8, we obtain the final balloons shown in Fig. 7.9. This figure serves two purposes. First, it shows that the combination method is effective in eliminating most of the redundant balloons. Second, in obtaining elongated structures, our binary balloons work well even in such a complex environment. With the balloons in place, we are now prepared to abstract the description of the street network and houses, as discussed next.

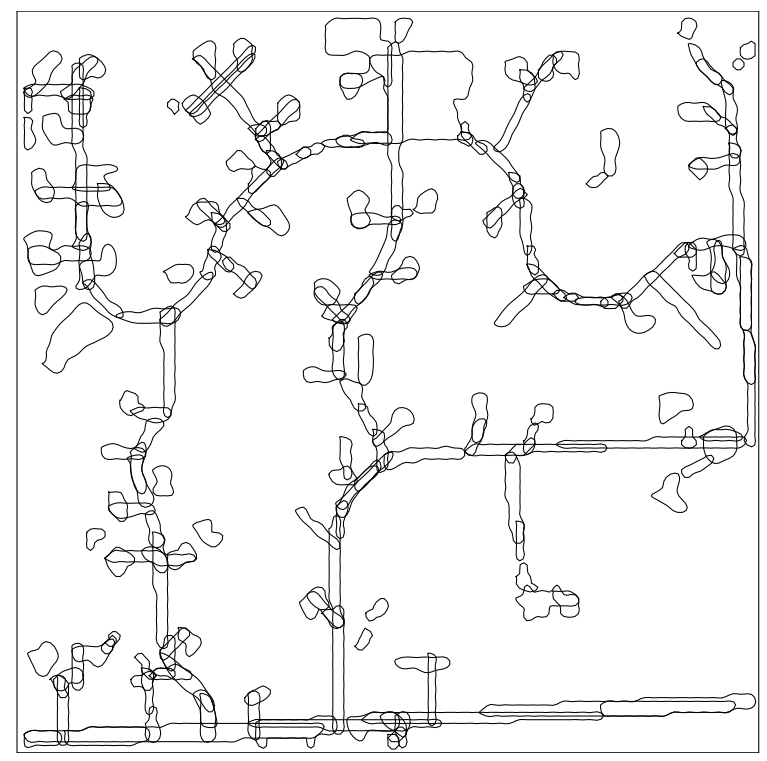

Figure 7.9: The South Dakota image, combination of balloons

\subsubsection{Abstracting the Scene: Attributed Balloons}

To build an abstract representation of the scene, we attribute each balloon with the following properties: boundary, spine, length (of the spine), center of mass, and 
average width. We represent the outer boundary of each balloon using Fourier descriptors. Let a complex periodic function, $u(t)=x(t)+j y(t)=u(t+r T), j=\sqrt{-1}$ for any integer values of $t$ and $r$, represent the outer boundary of the balloon. $T$ is the total number of points in the contour. The complex periodic contour can be approximated by a Fourier series as in Eqns. 3.4 and 3.5. Here, we use the fourth order Fourier series representation to filter the boundary shape prior to computing its features, below.

To obtain the spine of the balloon we use its curvature. The curvature is a differential geometric entity giving a measure of how rapidly the curve deviates from the tangent line [239]. We find the curvature of the filtered boundary $(\hat{u}(t)=\hat{x}(t)+$ $j \hat{y}(t))$ as:

$$
K(t)=\frac{\frac{d \hat{x}(t)}{d t} \frac{d^{2} \hat{y}(t)}{d t^{2}}-\frac{d \hat{y}(t)}{d t} \frac{d^{2} \hat{x}(t)}{d t^{2}}}{\left(\left(\frac{d \hat{x}(t)}{d t}\right)^{2}+\left(\frac{d \hat{y}(t)}{d t}\right)^{2}\right)^{3 / 2}}
$$

Given the balloon construction and filtering above, the extremal points of this curvature correspond to the endpoints of the spine (on the boundary) to be extracted. There is no algebraic solution to obtain the roots of Eqn. 7.5 directly; we solve it numerically.

Once we obtain the endpoints of the spine, we divide the contour function into two parts $x_{u}+j y_{u}$ and $x_{l}+j y_{l}$. These have the same parameter such that $k=\left[0, e_{2}-e_{1}\right)$ and 0 corresponds to $e_{1}, e_{2}-e_{1}$ corresponds to $e_{2}$ on the original (filtered) contour. Therefore, they have the same increment direction. The two parts have also been interpolated to have the same number of points. Our final spine curve, $s(k)$ for $k=\left[0, e_{2}-e_{1}\right)$ is: 


$$
s(k)=\frac{1}{2}\left[\left(x_{u}(k)+x_{l}(k)\right)+j\left(y_{u}(k)+y_{l}(k)\right)\right]
$$

To demonstrate the construction of the spine of a balloon, let's consider a horizontal balloon from the South Dakota image. We first obtain the filtered version of the boundary by a fourth order fit as in Fig. 7.10 (a). We give the corresponding curvature in Fig. 7.10 (b). As we find the extremal points of this curvature $\left(e_{1}, e_{2}\right.$ labelled on both boundary and the curvature). As can be seen, the extremal points of the curvature are distinct and easy to extract. We finally obtain the spine curve (represented by a dashed curve) in Fig. 7.10 (a).

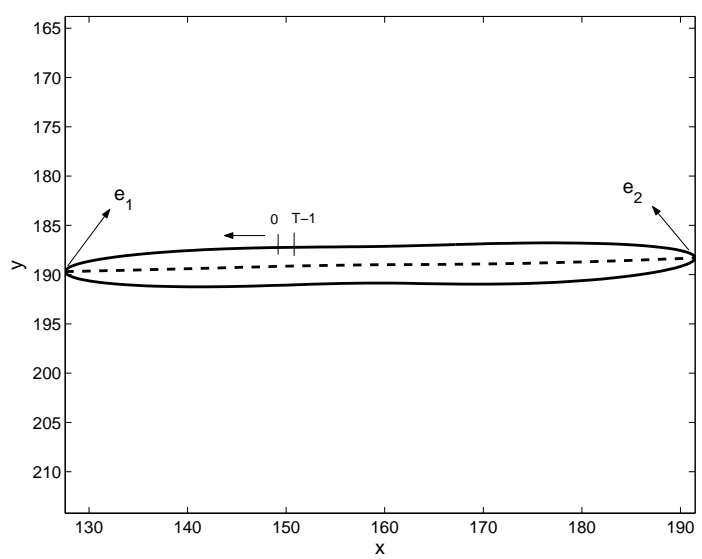

(a) FSR of the boundary and the spine extracted. solid curve - boundary, dashed curve - spine

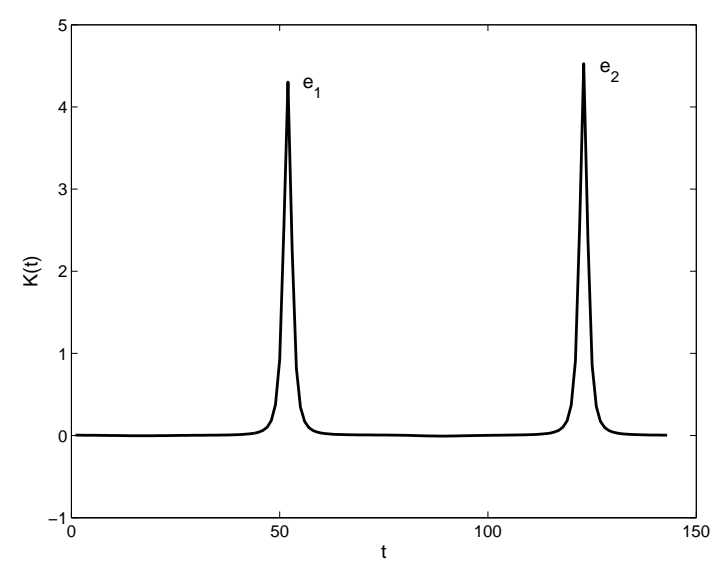

(b) The curvature of the boundary

Figure 7.10: Spine extraction example

We keep the boundary and the spine in parametric form for further processing (such as street extraction). We take the length of the spine as its arc length. The 
number of pixels on the spine could also be considered to be its length. However, the arc length is robust to non-uniform pixel placements along the spine. If the spine is given as $s(k)=x(k)+j y(k)$ then its arc length is:

$$
l=\int_{e_{1}}^{e_{2}} \sqrt{\left(\frac{d x(k)}{d k}\right)^{2}+\left(\frac{d y(k)}{d k}\right)^{2}} d k
$$

The center of mass of each balloon is calculated by the centroid of its point set. For the average width of the balloon, we extract the orthogonal lines to the spine (at its every pixel) ending at the boundary. The average length of these lines is the width of the balloon.

\subsection{Street Network and House Detection}

In this section, we extract the street network and houses from the balloons obtained. We first eliminate balloons showing neither street nor house characteristics. Then, we construct a graph over the remaining balloons. Using graph theoretical techniques and size information as discussed below, we jointly extract street networks and houses.

\subsubsection{Eliminating Balloons Corresponding to Large Struc- tures}

In residential regions we may also encounter large buildings such as shopping malls and schools, along with their parking lots. Therefore, some balloons produced in the previous step may represent these structures. We identify and discard these balloons by their morphology.

To discard balloons representing possible large buildings and parking lots, we use their area and compactness. Compactness is defined as the ratio of a region's area to 
the square of its perimeter, normalized by $4 \pi$ [235]. Hence, the most compact shape (circle) takes a value of 1 and the least a value of 0 .

Ours are metric images with the same resolution on the ground over the entire image set; so the area is a reliable feature. A typical house is far smaller area than a shopping mall or a parking lot. Road networks encompass large areas, of course, but they are highly non-compact. Therefore, we calculate the compactness as a second feature. Balloons possibly belonging to the street network have low compactness values. On the contrary, balloons possibly representing a house have high compactness values and their areas are small. So, we eliminate balloons having area $>75$ and compactness $>4 \pi / 60$ as belonging to neither the street network nor a house.

\subsubsection{Forming a Graph to Represent the Balloon Neighbor- hoods}

We extract the street network and houses using methods rooted in graph theory. We follow the same terminology as in chapter 6 . In this chapter, the weight between two adjacent vertices $(i, j)$ is $w(i, j)$ the distance between them (to be defined below). This weight increases with the increasing distance, such that larger weight implies a longer distance between two adjacent vertices.

We construct the balloon graph as follows. Each balloon is associated with a vertex in the graph. Therefore each vertex $v_{i}$ has three properties: arc length $l\left(v_{i}\right)$, width $w\left(v_{i}\right)$, and the center of mass $c\left(v_{i}\right)=(x, y)_{i}$. There is an edge between two vertices if their corresponding balloons have a common boundary section. The weight assigned to this edge is the distance between these two balloons. To calculate this distance let $v_{i}$ and $v_{j}$ be two adjacent balloons having a common boundary. Let the 
center of mass of the common boundary be $c_{c}=(x, y)_{c}$. The distance between these two balloons (the weight of the edge connecting them) is:

$$
w(i, j)=\left\|c\left(v_{i}\right)-c_{c}\right\|+\left\|c_{c}-c\left(v_{j}\right)\right\|
$$

Since we use distance as a weight, (and we will look at the shortest paths to construct the street segments) it is reasonable to use this definition. The above weight definition approximates the distance between the two adjacent vertices as good as possible. Next, we consider the distance between two (not necessarily adjacent) vertices.

Berge [179] defines the distance $d(i, j)$ between two (not necessarily adjacent) vertices $(i, j)$ in a weighted graph to be the length of the shortest path from vertex $i$ to vertex $j$.

Theorem 7.5.1 (Berge) $d(i, j)$ satisfies

$$
\begin{aligned}
& \text { 1- } d(i, i)=0 \\
& \text { 2- } d(i, j)+d(j, k) \geq d(i, k) \\
& \text { In addition if the graph is symmetric, we have }
\end{aligned}
$$

$$
\text { 3- } d(i, j)=d(j, i)
$$

This function is therefore a true metric in the topological sense.

We obtain the distance between any two vertices by means of Dijkstra's shortest path algorithm given in Alg. 5 [240]. This algorithm is one of the standard all shortest paths algorithms used in graph optimization problems. It can also be implemented in parallel, since at each step it finds the shortest path between any two given vertices. 


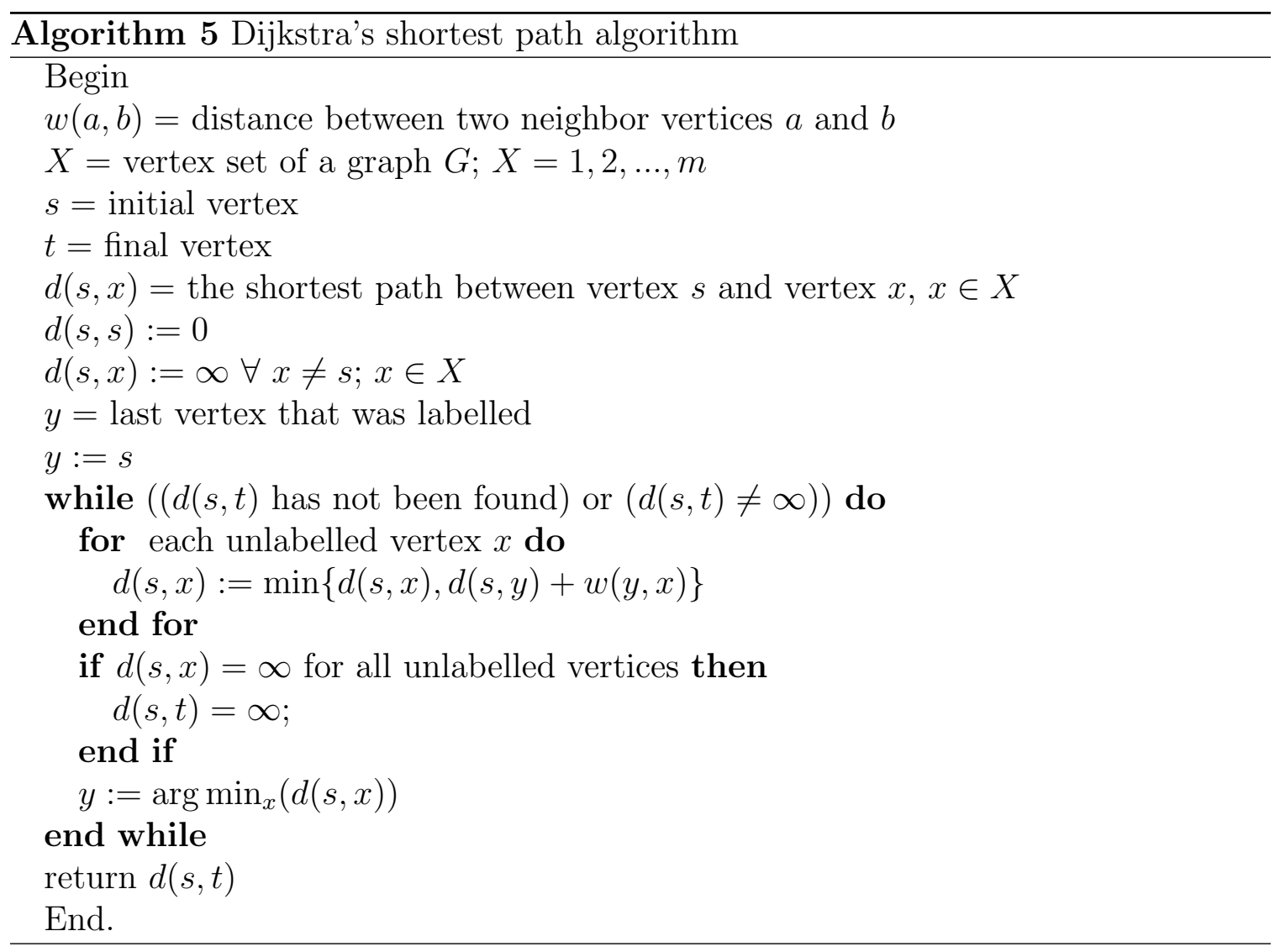

\subsubsection{The Detection Step}

Now, we turn our attention to the street network and house detection step. We introduce a novel algorithm based on graph theory. With this algorithm, we first extract the street network (via balloons) and label the remaining balloons as houses. To extract the street network, we have unary and binary constraints. Unary constraints are used to detect balloons those can represent a street segment themselves. Binary constraints are used for the balloons those can represent a street segment only with the help of their neighbors.

We assume that a balloon represents a part of a house or a street segment at this step, based on their construction, and limitations applied to it. There are general 
house and street characteristics (at least for North America) that lead to a street network and house detection algorithm from the balloon graph. These are as follows.

- In residential regions, houses are connected to the street network via driveways. Since we take driveways as belonging to the street network, houses are located at its endpoints. In some cases short, wide driveways may be assigned to the street network. This is not a major problem.

- Compared to an elongated street segment, the aspect ratio of a house is small. Therefore, a ratio threshold can help in discriminating house and street balloons to a great extent.

- A street network is continuous, with straight and curved sections. Balloons obtained in these sections will be elongated and few for the straight sections; small and more for curved sections. The balloons of the street network are connected, because the network is connected.

We apply these observations to obtain the street network and houses from the balloon graph. For each vertex (balloon) we calculate its aspect ratio. This ratio will indicate the likelihood of that balloon belonging to a street network. We take balloons having a ratio greater than 7.5 to be street segments. This is (initial) street segment extraction by unary constraints.

We then look at binary constraints to complete the extracting of the street network. We first discard any vertex of aspect ratio smaller than $t_{r}$ and having at most one neighbor. As we mentioned, such vertices are more likely to represent a house than a street segment. For vertices (balloons) having more than two adjacent vertices 
to belong to a street segment, their combination should exhibit the geometric characteristics of a street. Therefore, we retain paths of balloons (each member has at least two neighbors and an aspect ratio of at least five), having distances between their furthest vertices to be larger than a threshold $t_{d}$ (taken as five pixels, or 20 meters here). The underlying assumption is that a path with more than 20 meters length is more likely to be a street segment than a house group. Again we have metric images, so constant thresholds are applicable. In curved sections of the street network, we will have many balloons with more than one neighbor and aspect ratio less than five, but by chaining them together we can extract the street(s). The balloons not belonging to the street network are labelled as houses.

This algorithm may seem simple; however it should be understood as a portion of a larger system. We have restricted our choices either to a street segment or a house through the earlier processing. We have also restricted the region we are working on to be residential. These regions have strict rules in terms of house and street locations, such that houses and street networks lie in close proximity, and a street can not pass through a house. Therefore, this relatively simple graph theoretical street network extraction method works fairly well. The street network and house detection algorithm is given in Alg. 6 .

\subsubsection{Road Tracking by Prediction}

Some street segments may be missing by occlusion (such as overhanging trees), be labelled as houses, or be eliminated due to their size. To recover these missing street segments, we apply a prediction based road tracking method. Our method is similar to the correlation based road tracking methods summarized in [241]. 


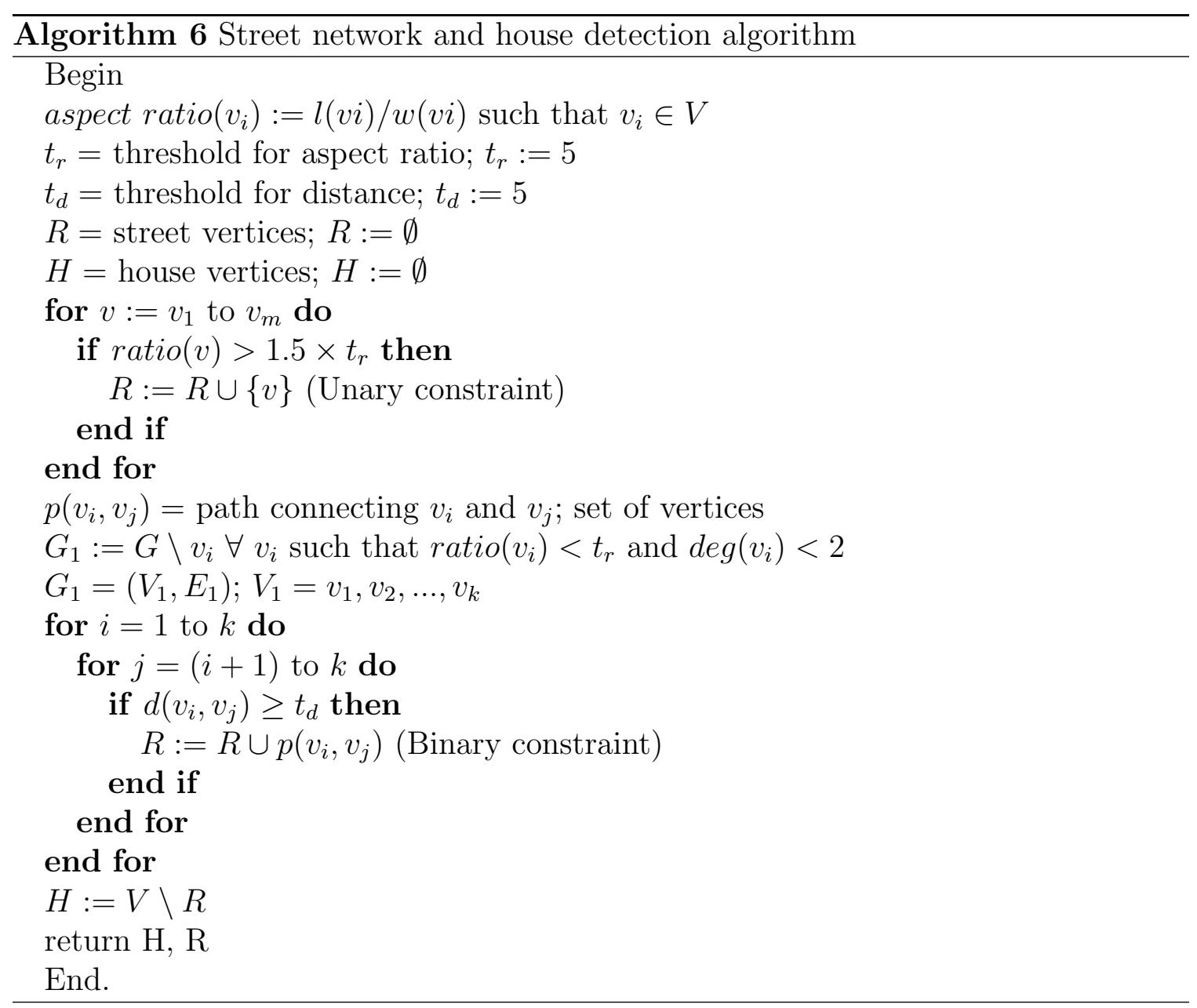


We apply the road tracking by prediction only to those street balloons having a single neighbor $\left(\operatorname{deg}\left(v_{i}\right)=1\right)$. Therefore, we track possible streets only from endpoints of the balloon graph. We recall the parametric representation of their spines and for each spine representation we apply the following method.

Let $r(t)=(x(t), y(t))$ be the the spine of a balloon with $\operatorname{deg}\left(v_{i}\right)=1$. It is represented by a $k^{t h}$ order polynomial on $n$ points. $x(t)=\sum_{i=0}^{k} a_{i} t^{i}, y(t)=\sum_{i=0}^{k} b_{i} t^{i}$ $t=[1,2, \ldots, n] . \quad a_{i}$ and $b_{i}$ are obtained from the least squares fit. For this study we take $\mathrm{k}$ to be one.

Our prediction of the next point will be $\hat{r}_{1}(n+1)=(\hat{x}(n+1), \hat{y}(n+1))$; extrapolating the polynomial fit. We also pick two neighboring pixels to $\hat{r}_{1}(n+1)$ orthogonal to the spine curve, as $\hat{r}_{2}(n+1)$ and $\hat{r}_{3}(n+1)$. We obtain the next street point by $\hat{r}(n+1)=\arg \max _{\hat{r} \in\left\{\hat{r}_{1}, \hat{r}_{2}, \hat{r}_{3}\right\}} \Omega\{\hat{r}\}$ where $\Omega$ is an index of human activity (Eq. 7.1) . We then take the most recent $n$ points, fit a polynomial to them, and repeat the prediction. Tracking is continued until $\max _{\hat{r} \in\left\{\hat{r}_{1}, \hat{r}_{2}, \hat{r}_{3}\right\}} \Omega\{\hat{r}\}$ is smaller than a threshold $t_{p}$, discussed next.

We want road tracking to continue only for pixels having sufficiently high $\Omega$. Therefore, we calculate $t_{p}$ in a Bayesian decision framework between object (high $\Omega$ values, $C^{o}$ ) and background (low $\Omega$ values, $C^{b}$ ) classes for each image separately ( $t_{p}$ is therefore adaptive). We first obtain the conditional sample $\Omega$ distributions for object and background classes. We then set $t_{p}$ equal to the optimal Bayesian decision boundary value between these two classes. Rarely, this threshold may not be sufficiently strong to stop iteration. To handle these cases, we insert a control, checking the length of the extracted street segment. 
We illustrate this method on the South Dakota image in Fig. 7.11. Although most of the street segment balloons have been labelled correctly, some were discarded while combining balloons and applying size constraints. One of the actual street segment balloons is also labelled as a house. To correct these errors, we apply the road tracking algorithm. Fig. 7.11 (b) presents the street network extracted before tracking in green. The street network as extended by the road tracking algorithm is given in red. As can be seen, almost all missing street segments have been recovered by the tracking method.

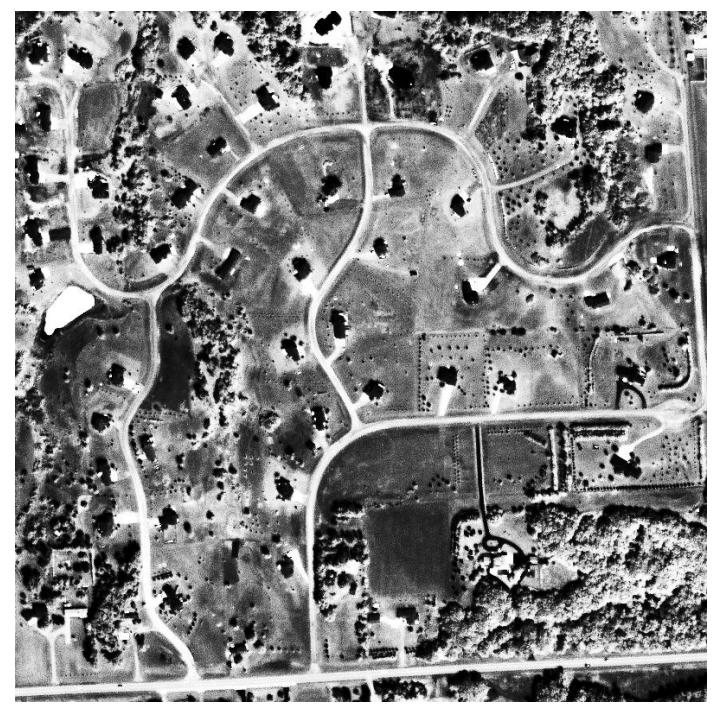

(a) The South Dakota image

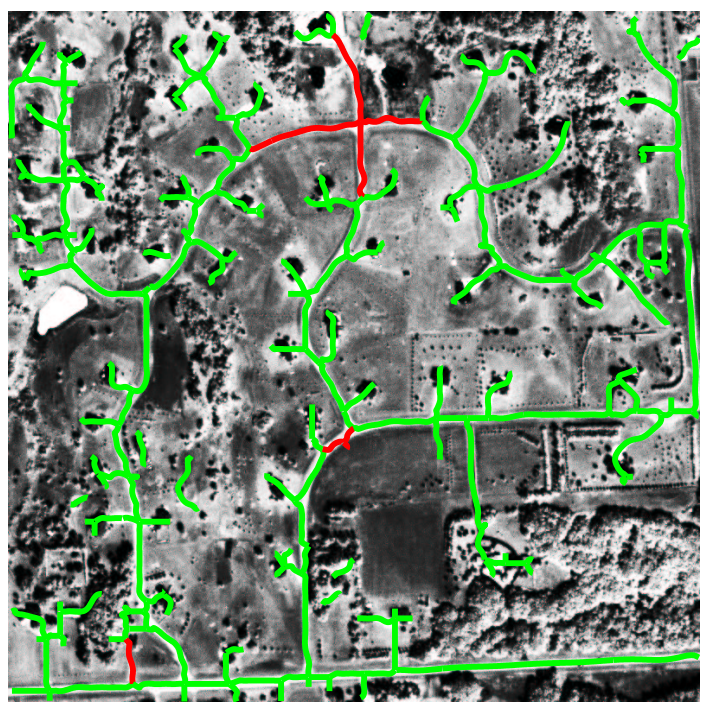

(b) The final road network

Figure 7.11: The South Dakota image, street network extraction. Green sections are obtained by the graph theoretical method; red sections are obtained by road tracking 


\subsection{Results and Discussion}

We tested our methods on 44 residential images (each panchromatic image being $800 \times 800$ pixels, with $200 \times 200$ multispectral representations) taken from different locations around North America.

We first tested the k-means clustering with spatial coherence to extract possible house and street network pixels. Being the initial step, our house and street detection methods depend directly on it. We applied a pixel based classification to evaluate this method. Next, we evaluated the house and street network detection steps separately. We provided four examples to show the performance of our system on four different residential region types.

\subsubsection{Pixel based Classification}

In classification, we take house or street pixels $\left(C^{o}\right)$ as one group and the background pixels $\left(C^{b}\right)$ as another group. We have $44 \times 200 \times 200$ pixels since we are using the multispectral representation. We obtain $97.8 \%$ and $94.5 \%$ correct classification rates for the background and house or street pixels with an average classification performance of $\mathbf{9 7 . 3} \%$. The errors in this section are mostly due to overhanging trees blocking street segments and houses.

\subsubsection{House Detection}

Next, we evaluate the house detection in residential regions. In our image set, we have a total of 6803 houses. Depending on the region, houses have different characteristics. Size, shape, and the setback from the street all vary, as does the spacing between them. 
To evaluate house detection performance, we report probability of detection $P_{d}$ and branching factor $B_{f}$ as in Table 7.2. Here, we assume that a house is detected if any part of it is detected as in [216]. We obtain $\mathbf{P}_{\mathbf{d}}=\mathbf{9 2 . 9} \%$ with $\mathbf{B}_{\mathbf{f}}=\mathbf{9 . 5} \%$ under these circumstances. This result indicates the effectiveness of our house detection methodology.

\subsubsection{Street Network Detection}

Finally, we evaluate the street network detection step. In our residential test images, the total length of all 44 street networks is 55442 pixels $(55442 \times 4$ meters $)$. Similar to Table 7.4, we provide $P_{d}$ and $P_{f}$. Although we have the width information for the street segments, we only consider the $P_{d}$ in terms of length. In this section, we also evaluate the quality of street tracking by the prediction technique.

We applied our street detection algorithm and obtained $P_{d}=89.9 \%$ with $P_{f}=$ $3.8 \%$ before prediction. Road tracking increased both: $\mathbf{P}_{\mathbf{d}}=\mathbf{9 4 . 8} \%, \mathbf{P}_{\mathbf{f}}=\mathbf{8 . 0} \%$. It improved $P_{d}$ by $4.9 \%$, at the cost of increasing $P_{f}$ by $4.2 \%$. Although $P_{f}$ increased substantially (decreasing the system performance), improvement in the $P_{d}$ is more important, since our aim is to detect street networks reliably. Therefore, tracking by prediction is a necessary part of the system.

\subsubsection{Some Detection Examples}

We present the house and street network detection results for four images (Indiana, Maryland, South Dakota, and South Dakota II). Next, we focus on each image, to observe the performance of house and street network detection system. To demonstrate house and street network detection performance we followed the same color code for both (green corresponds to a correct detection, blue corresponds to a miss, 
and red corresponds to a false alarm in subfigures (b) and (c)). We also provided the overall detection for each image (in subfigures (d)).

The first result is on the Indiana image (Fig. 7.12). This residential region represents three distinctive characteristics. It has a mature region with well-spaced houses and trees nearby. There is a lake in the middle of the scene. The region in the upper left is a construction zone with new houses. Therefore, this scene is one of the hardest to process. There are 170 houses in the scene of which 165 of them are correctly localized with 12 false alarms. False alarms originate mostly from the spacing between houses and street segments. Some locations near street segments are falsely recognized as houses. Houses in the mature area are difficult to detect even for a human observer. Our system was able to locate most of these houses. The 1525 pixel length street network is extracted except for a 10 pixel section, with a 90 pixel false alarm. At the road tracking step, tracking extended beyond driveways for one or two pixels because of dense location of houses and street segments, causing most of the false alarms. The missing street segment could not be recovered by road tracking, since some portions of it were covered by overhanging trees. The remaining street network is correctly detected with high accuracy for this scene.

The Maryland image (Fig. 7.13) represents another type of residential region. It has well-spaced and similar houses. Although there are trees in the scene, they are not close to the houses. This indicates that the neighborhood is newly constructed in a previously forested region. There are 151 houses in the scene, of which 145 are correctly located, with three false alarms. Since we have well-spaced houses compared to the Indiana image, the false alarm rate is low. For this image, the 1133 pixel street 


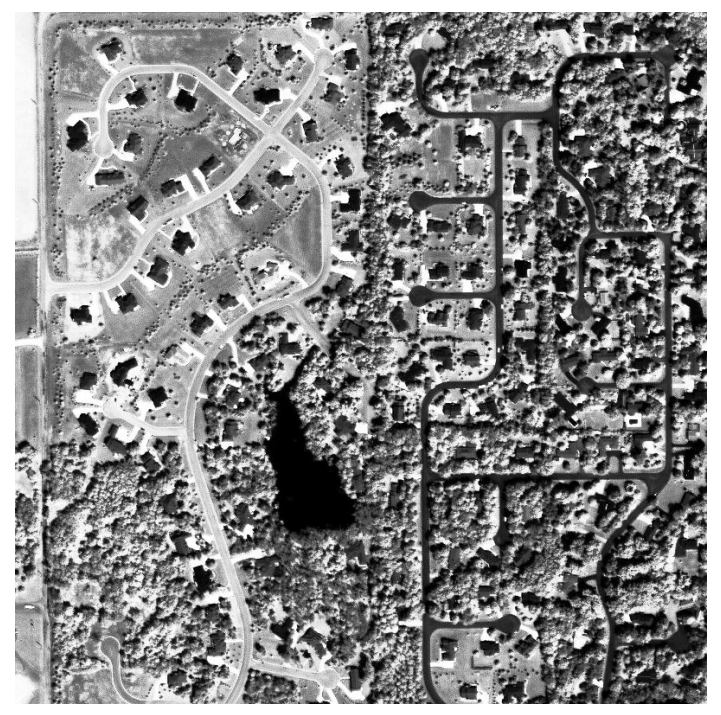

(a) The panchromatic image

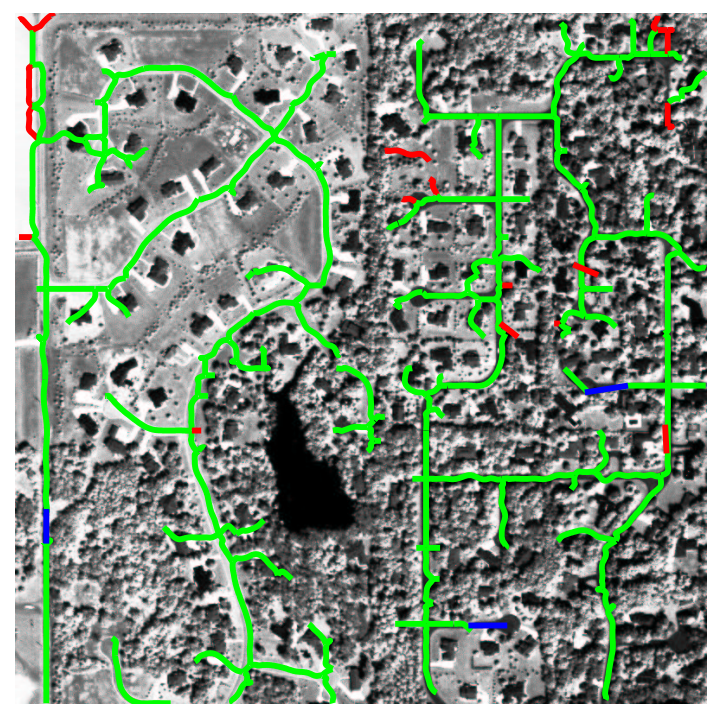

(c) Street network detection, green - correct, blue - miss, red - false alarm

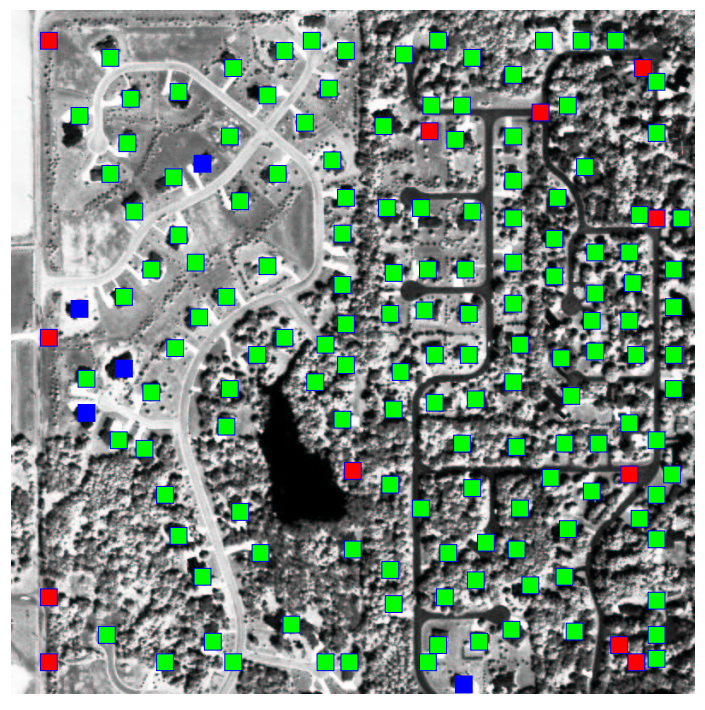

(b) House detection; green - correct, blue - miss, red - false alarm

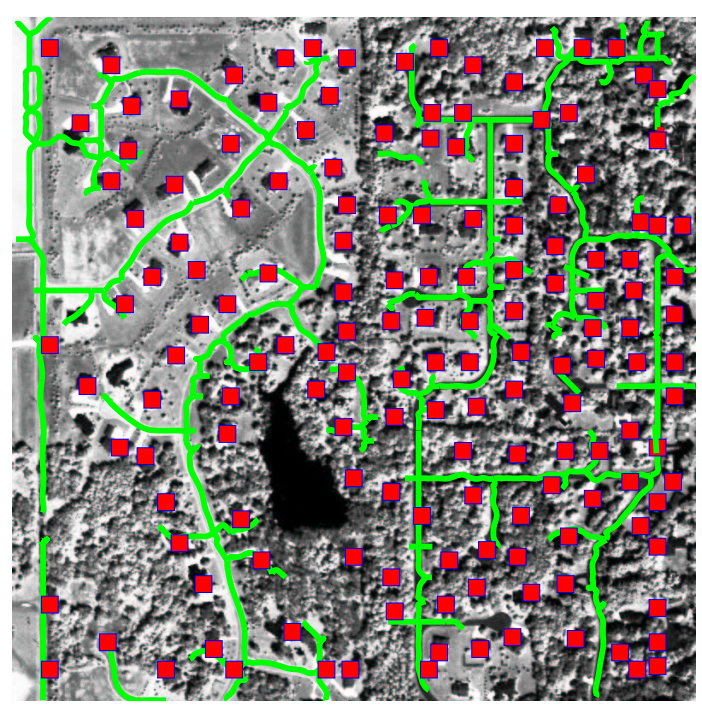

(d) The overall detection, green - street network, red - house

Figure 7.12: The Indiana image, street network and house detection 
network is extracted except for a 46 pixel section. The majority of the missing street section is at the upper part, where it is occluded by trees.

The third result is on the South Dakota image (Fig. 7.14). This residential region represents low density housing, with only occlusions of the houses and streets. There is also little vegetation in the scene. Almost all houses and the street network can be seen clearly. This region is one of the easiest to process. In this residential region with 69 houses, 67 are correctly located with one false alarm. 1258 pixels of the 1276 pixel street network are detected correctly, with a 73 pixel false alarm. Although road tracking was able to recover most of the missing segments for this image, it also increased the false alarm rate, mostly because of the low vegetation cover (causing extra one or two iterations).

The South Dakota II image (Fig. 7.15) is similar to the Indiana image. It is composed of both mature and newly constructed regions. Although there are fewer houses in the scene, the occlusion by overhanging trees is more prevalent than in the Indiana image. The spacing of the houses is similar to that of the Maryland image. For this region, 141 of 147 houses are correctly located, with six false alarms. The 1411 pixel length street network is detected with 193 pixels missing. Two main street segments, the first being at the top of the scene, the second being at the middle. The false alarm count for street extraction in this image is 68 pixels.

As we observe these four results, we can comment on possible weaknesses of our system. First, as the houses in a region becomes more densely spaced, the house and street network detection performance decreases. This is to be expected. Road tracking increases the false alarm rate considerably in street detection. Our detection system can not overcome significant occlusion by trees. Therefore, if a house or 


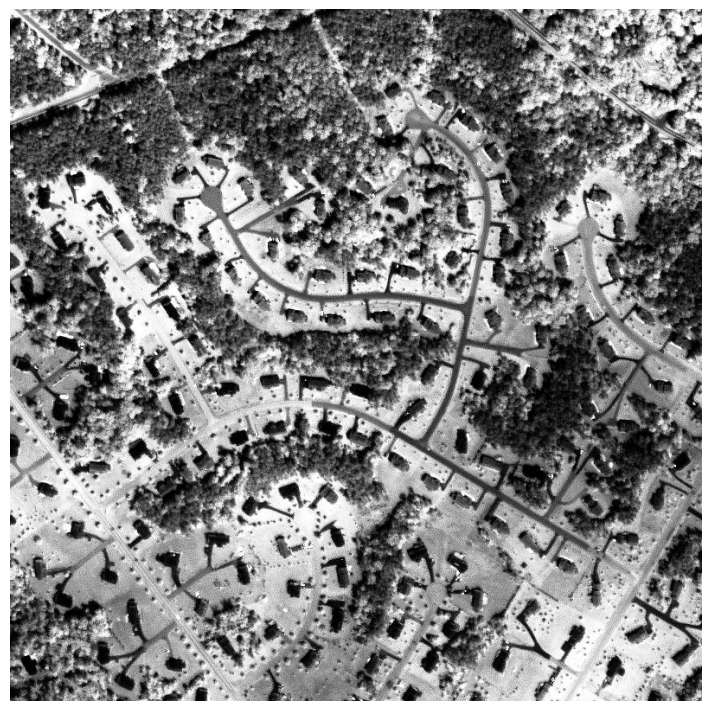

(a) The panchromatic image

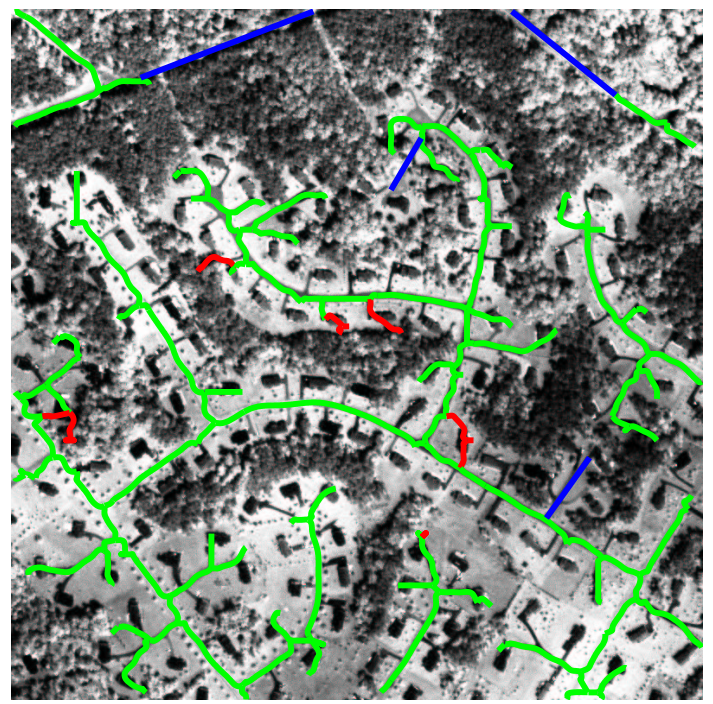

(c) Street network detection, green - correct, blue - miss, red - false alarm

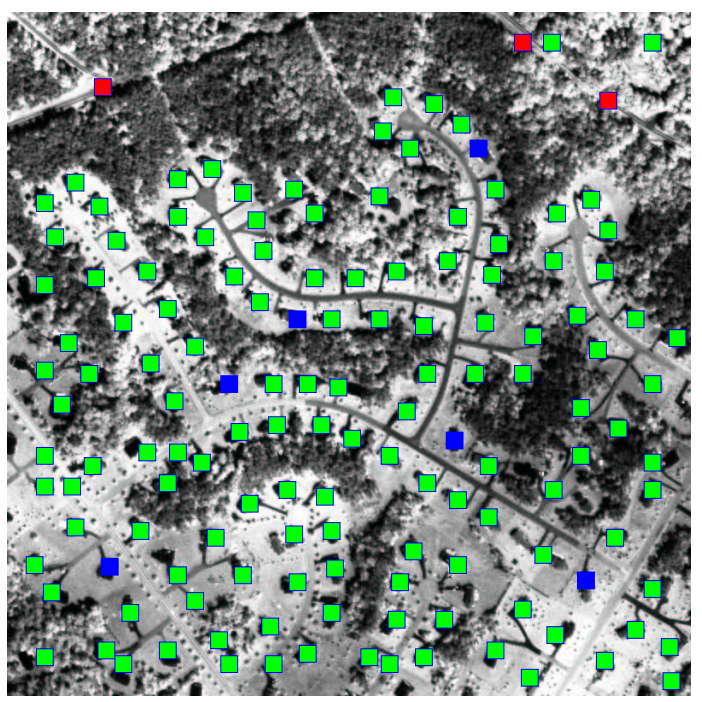

(b) House detection; green - correct, blue - miss, red - false alarm

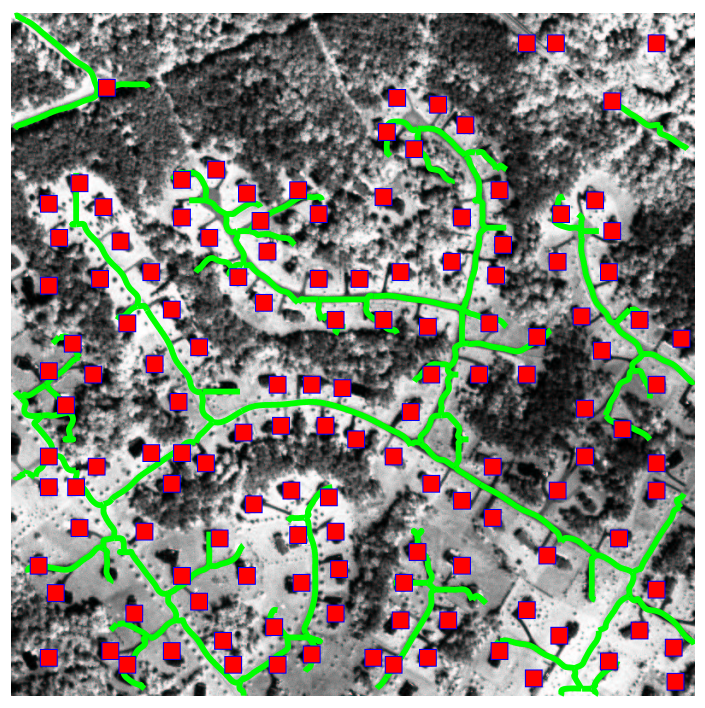

(d) The overall detection, green - street network, red - house

Figure 7.13: The Maryland image, street network and house detection 


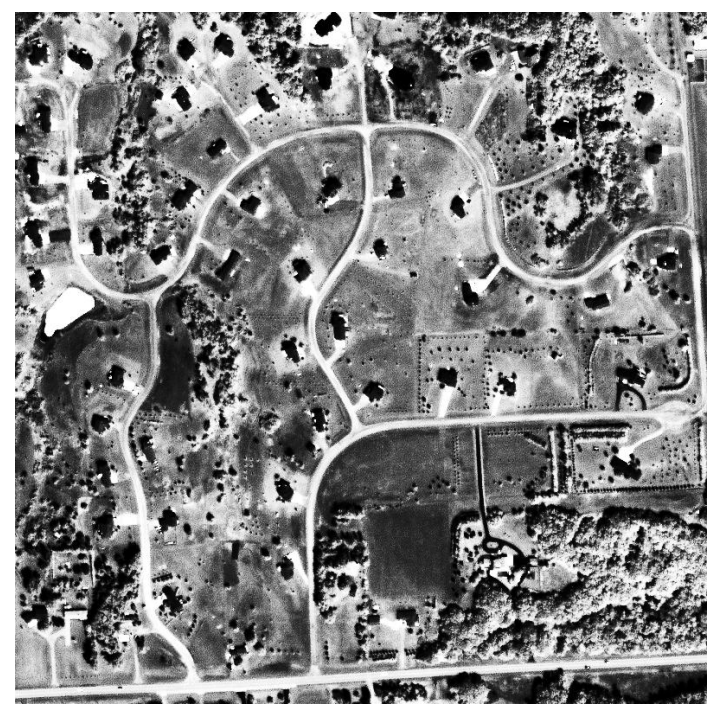

(a) The panchromatic image

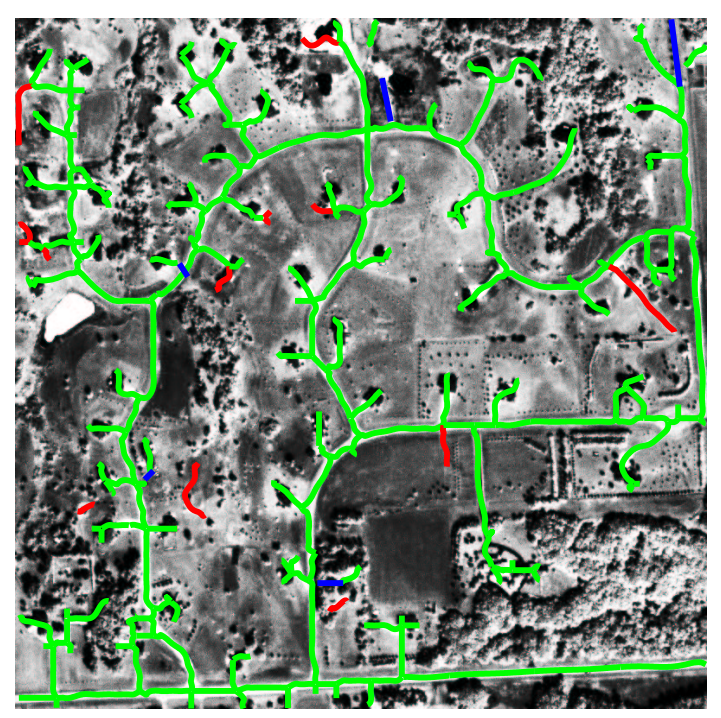

(c) Street network detection, green - correct, blue - miss, red - false alarm

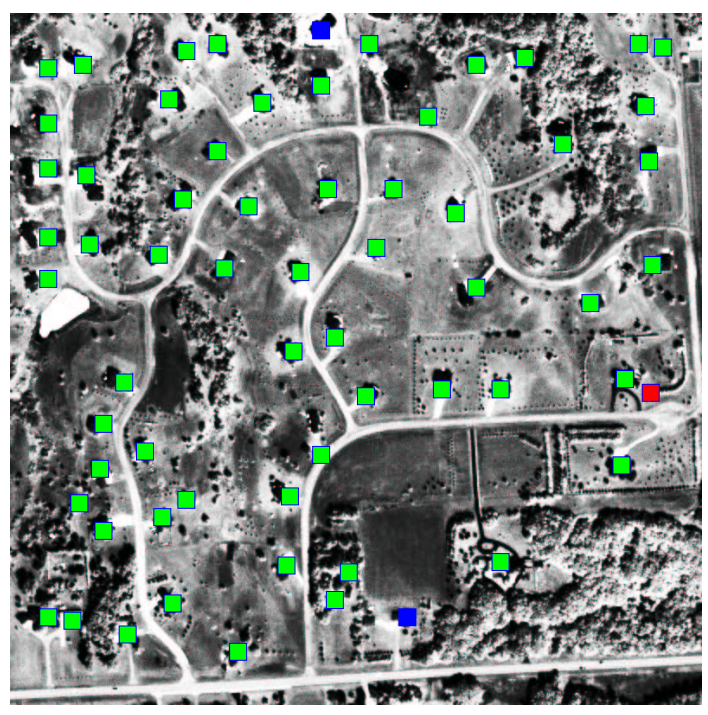

(b) House detection; green - correct, blue - miss, red - false alarm

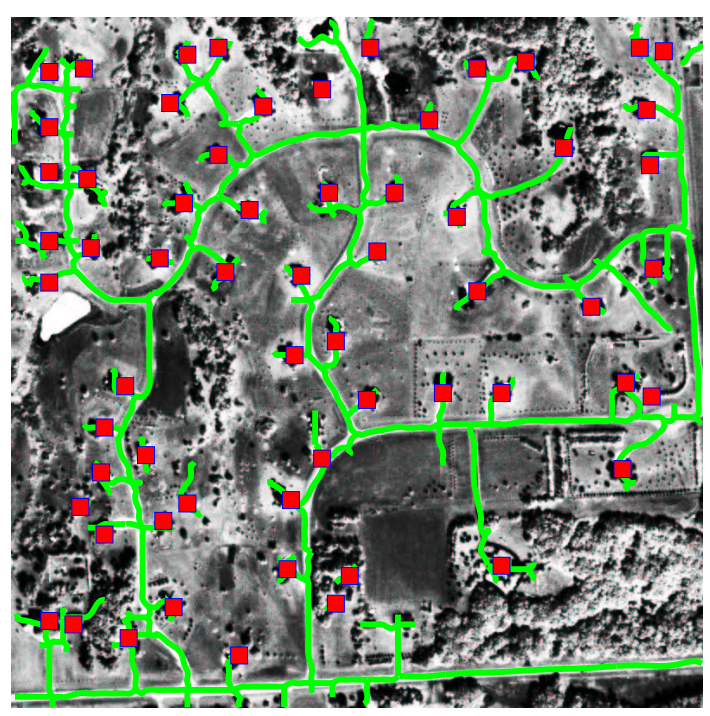

(d) The overall detection, green - street network, red - house

Figure 7.14: The South Dakota image, street network and house detection 


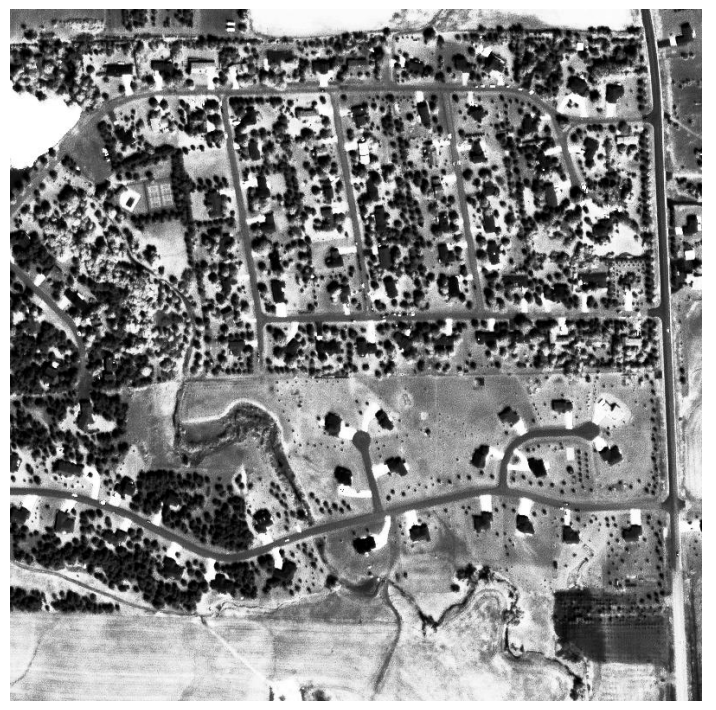

(a) The panchromatic image

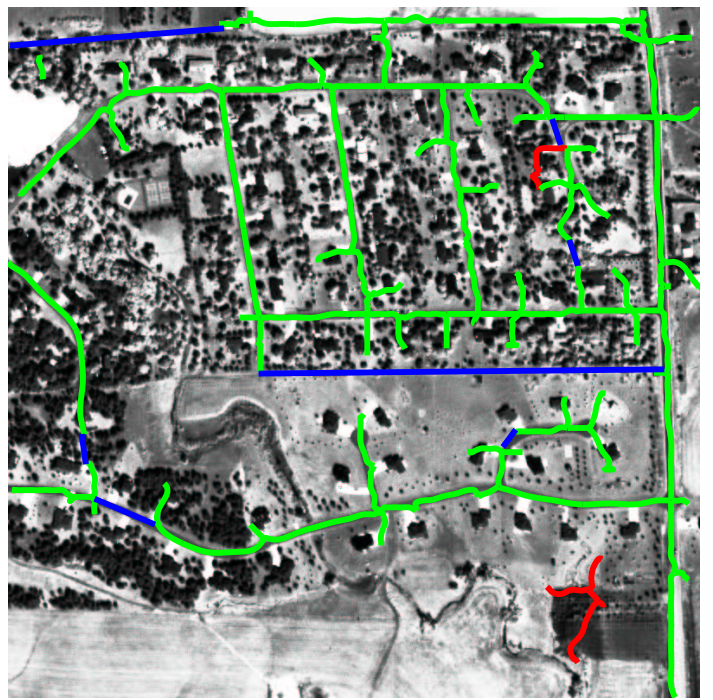

(c) Street network detection, green - correct, blue - miss, red - false alarm

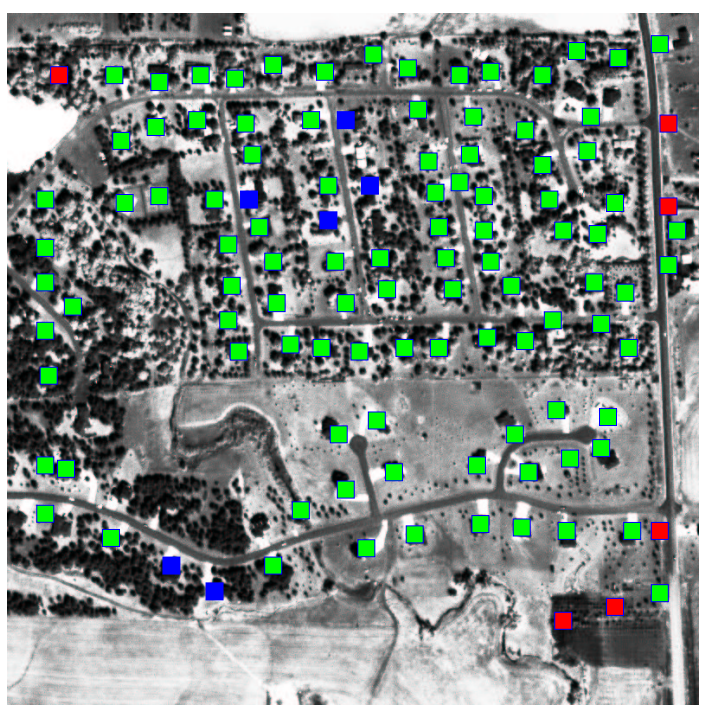

(b) House detection; green - correct, blue - miss, red - false alarm

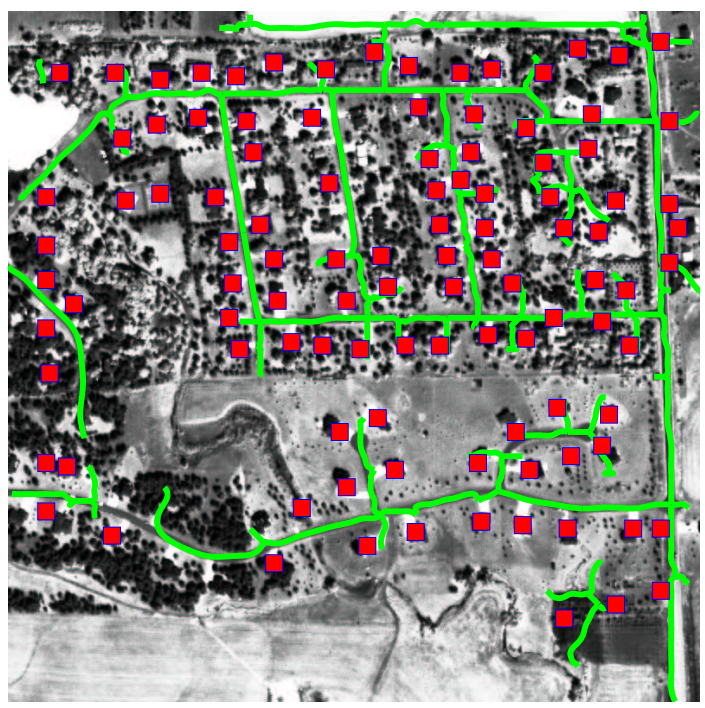

(d) The overall detection, green - street network, red - house

Figure 7.15: The South Dakota II image, street network and house detection 
a street segment is totally occluded by trees, it won't be detected. This could be probably addressed by introducing a higher-level reasoning process to infer missing street segments, but such a process would not be simple, and lies beyond the scope of this paper.

These are the minor problems the system reveals. On the other hand, these four different types of regions show the robustness of our house and street detection system to different conditions. In these four images, most of the houses and street network were detected correctly. For the Indiana and South Dakota II images it is extremely difficult for a human observer to detect the houses in the mature (wooded) sections. Our system was able to detect and locate most of the houses even in these regions correctly.

\subsection{Summary of the Chapter}

In this chapter, we introduced a system to detect street networks and houses in residential regions via satellite images. We started from the multispectral images, introduced an extension of the k-means clustering to segment out possible human and street network pixels in the image. To extract houses and street networks on this segment, we introduced a decomposition algorithm inspired by balloon algorithms. We call this decomposition algorithm as binary balloons. Then we represented these balloons in a graph and applied graph theoretical methods to extract street networks and houses. We tested this system on a diverse data set. The results indicate the functionality of it. 


\section{CHAPTER 8}

\section{Conclusions}

We first reviewed the literature to observe the trends and performances in land use classification. Since, most of the papers reported performances over a single image, we could not reach a conclusion on their relative performances. However, we observed that none of these studies were based on the structural properties of the grayscale images which we believe is the most promising direction for the publicly-available high resolution satellite images.

To that end, we first presented a new approach to assess the degree of land development. While spectral signatures have been exploited for years in land use classification, our objective was to learn what information regarding land development could be extracted - efficiently - from the photometric structure in the image. We based the assessment on the photometric and geometric characteristics of straight line segments. Although edge detection may be used to extract lines, we used a more robust straight line extraction method based on regions of consistent gradient orientation.

Among several features we developed, those based on length and contrast proved to be the most promising for classification. The strength of these features is that they do not depend heavily on the city model; they apply to most cities around the world, particularly over $400 \mathrm{~m}^{2}$ windows. The weaker features are those more dependent on 
a specific city model as described in the Appendix. The model assumes a high(er) degree of organization, which may not hold for many old world cities. However, if one's focus were on urban and rural discrimination in North America (and Australia, for example), then these features may prove more useful.

We tested both parametric (Bayes) and non-parametric (Parzen window and nearest-neighbor) classifiers. Although these classifiers differ in their assumptions with respect to the underlying data distribution, they offer similar performance on our data. However, once trained, the Bayes classifier requires the least computation; nearest neighbor the most. Therefore, we would recommend the Bayes classifier for a production system using this type of data. With probabilistic relaxation we obtained slightly improved classification performance. However, this improvement is marginal considering its additional computational cost and we would not recommend it.

The detection of suburban residential regions is highly challenging. Nevertheless, a novel feature based grouping method produced highly encouraging results. This suggests that methods based on principles from perceptual organization may ultimately have significant impact on these types of problems.

Although our test set is large and diverse in location and development pattern, we can distinguish developed areas with high confidence. Our results indicate that image structure, as captured by the spatial organization of its straight lines, does provide an effective indicator of land development activity.

To extend these measures to multispectral images, we considered the best known vegetation index in the remote sensing literature, NDVI, and developed a rigorous statistical justification for it by using principal components analysis. As we observed, the NDVI in fact corresponds to a slope in the statistically decorrelated vector space. 
Armed with this interpretation, we then proposed a solution to the saturation problem of the NDVI. Simulations and experiments on real images show that our proposed measure overcomes the saturation problem.

Next, we considered the relationship between the $N D V I$ and the $R V I$ and showed that these two indices in fact capture the same information. With the statistical framework at hand, we considered more bands and introduced new vegetation and shadow-water indices. We compared these new vegetation indices with the angle measure we derived from the NDVI on real images. We conclude that while all of the indices introduced can be used to detect vegetation successfully none clearly outperforms $\theta$, based on the original $N D V I$ ratio. We also compared shadow-water indices on real images and found that $\gamma_{2}$ based on the blue, red and near-infrared bands works best.

By the help of this multispectral information, we improved our previous results in discriminating urban and non-urban areas in three steps. Our first step was to include the linearized NDVI as multispectral information. Our second step was to synergistical combination of the structural and the multispectral information. Our last step was using the spatial information in classification. Our most promising features are those using the combination of structural and multispectral information. By using parametric and non-parametric classifiers we were able to distinguish developed areas with high confidence.

Next, we introduced and tested several graph theoretical measures to assess land development in overhead imagery. Our first objective was to develop measures that increase monotonically with respect to the organization (development) in images. The third measure on graph partitioning performed better among others, in terms of false 
alarm rate. The main reason for this is the contrast information it has. Contrast based other structural features also proved to be useful as structural features.

Although each measure performed fairly well alone, the measure obtained by fusing them performed best of all with respect to deviation (this measure matches the human opinion quite well). It captures different properties of the scene by different measures, and these seem to compensate each other.

Our second objective was detecting sparse residential regions, which are difficult to discriminate. We first cast this as a one class problem and tested it with 281 samples. We obtained a clear and definite improvement by using graph based features over structural ones. This indicates that for fine classification, structural features alone are not enough. Besides the structure in the scene, neighborhood information of these structures (captured by a graph here) are also needed.

Finally, we built a three class classifier and tested it with 270 samples. For the three class case, we obtained classification results superior to those reported anywhere over such a number of test samples. Again, this test indicates the necessity of graph theoretical measures for fine classification. With these very high detection rates, our approach can be used to detect residential regions automatically.

Then, we introduced a system to detect street networks and houses in extracted residential regions. Our system comprises four parts: multispectral information processing, segmentation, decomposition, and graph theoretical analysis.

We started from the multispectral images, to locate possible human activity. We used the properties of $\theta$ for this purpose. This index (and the NDVI, of course) responds low to rock derivatives. Since these are the basic blocks for most of the houses and streets in residential regions, their presence would indicate possible human 
activity. And this was the case for our test images. Unfortunately, lakes and shadows also respond like rocks to this index. To overcome this problem, we introduced a shadow-water index, and then eliminated only water regions. This human activity index, as well as water region extraction method can be used for applications other than ours.

Having successfully identified the areas of human activity, we introduced a modified $\mathrm{k}$ means clustering algorithm to extract a binary segment representing possible houses, street networks, schools, malls, and their parking lots. The novelty in our algorithm is its introduction of spatial coherence to clustering via connected components analysis.

To extract houses and street networks on this binary segment, we introduced a decomposition algorithm inspired by balloon algorithms. Our aim was to extract elongated structures (representing possible street segments) via this algorithm. To represent the binary image reliably (covering the curved regions, specifically) we have multiple balloon representations for the same region. We were able to overcome redundancy by applying a voting method. This overall scheme worked fairly well in decomposing binary images we have.

Then we represented these balloons in a graph framework to extract street networks and houses. At this step we eliminated balloons representing neither a street nor a house structure. Therefore, at this step, a balloon in the graph is either representing a house or a street segment. By unary and binary constraints, we were able to detect houses and street networks on this graph.

We tested our house and street network detection system with 44 residential images. Our performance on such a diverse and large test set is noteworthy. We plan 
to use the spatial constraints (formalizing them as of either probabilistic relaxation or Bayesian networks) to refine our house and the street network labels. The overall system hence may be of use as an automatic map generation system focused on residential regions.

Finally, the overall system works fairly well, as we provided the statistical performance of each step. This system may be used to generate maps labelling houses and road networks in residential regions, as well as locating these neighborhoods. 


\section{BIBLIOGRAPHY}

[1] G. Konecny and J. Schiewe, "Mapping from digital satellite image data with special reference to MOMS-02," ISPRS Journal of Photogrammetry \& Remote Sensing, vol. 51, pp. 173-181, 1996.

[2] K. S. Fu, D. A. Landgrebe, and T. L. Philips, "Information processing of remotely sensed agricultural data," Proc. IEEE, vol. 57, pp. 639-653, 1969.

[3] D. A. Landgrebe, "The development of a spectral-spatial classifier for Earth observational data," Pattern Recognition, vol. 12, pp. 165-175, 1980.

[4] S. Khorram, J. A. Brockhaus, and H. M. Cheshire, "Comparison of Landsat MSS and TM data for urban land use classification," IEEE Trans. on GeoRS, vol. 25, no. 2, pp. 238-243, March 1987.

[5] J. P. Gastellu-Etchegorry, "An assessment of SPOT XS and Landsat MSS data for digital classification of near urban land cover," International Journal of Remote Sensing, vol. 11, pp. 225-235, 1990.

[6] D. Lloyd, "A phenological classification of terrestrial vegetation cover using shortwave vegetation index imagery," International Journal of Remote Sensing, vol. 11, pp. 2269-2279, 1990.

[7] R. S. DeFries and J. G. R. Townshend, "NDVI derived land cover classifications at a global scale," International Journal of Remote Sensing, vol. 15, pp. 35673586, 1994.

[8] J. P. Hoffbeck and D. A. Landgrebe, "Classification of remote sensing images having high spectral resolution," Remote Sensing of Environment, vol. 57, pp. 119-126, 1996.

[9] S. Bandyopadhyay and S. K. Pal, "Pixel classification using variable string genetic algorithms with chromosome differentiation," IEEE Trans. on GeoRS, vol. 39, no. 2, pp. 303-308, February 2001.

[10] R. M. Haralick, "Statistical and structural approaches to texture," Proceedings of the IEEE, pp. 786-804, 1979. 
[11] M. Tuceryan and A. K. Jain, "Texture analysis," The Handbook of Pattern Recognition and Computer Vision,92, pp. 235-276, 1993.

[12] J.S. Weszka, C.R. Dyer, and A. Rosenfeld, "A comparative study of texture measures for terrain classification," IEEE Trans. on SMC, vol. 6, no. 4, pp. 269-286, April 1976.

[13] J. R. Irons and G. W. Petersen, "Texture transforms of remote sensing data," Remote Sensing of Environment, vol. 11, pp. 359-370, 1981.

[14] R. W. Conners, M. A. Trivedi, and C. A. Harlow, "Segmentation of a high resolution urban scene using textural operators," Computer Vision, Graphics and Image Processing, vol. 25, pp. 273-310, 1984.

[15] R. M. Haralick and K. Shanmugam, "Computer classification of reservoir sandstones," IEEE Trans. on Geoscience Electronics, vol. 11, pp. 171-177, October 1973.

[16] R. M. Haralick, K. Shanmugam, and I. Dinstein, "Textural features for image classification," IEEE Trans. on SMC, vol. 3, pp. 610-621, 1973.

[17] D. J. Marceau, P. J. Howarth, J. M. M. Dubois, and D. J. Gratton, "Evaluation of the grey level co-occurrence matrix method for land cover classification using SPOT imagery," IEEE Trans. on GeoRS, vol. 28, no. 4, pp. 513-, July 1990.

[18] J. H. Lee and W. D. Philpot, "Spectral texture pattern matching: A classifier for digital imagery," IEEE Trans. on GeoRS, vol. 29, no. 4, pp. 545-554, July 1991.

[19] E. Sali and H. Wolfson, "Texture classification in aerial photographs and satellite data," International Journal of Remote Sensing, vol. 13, pp. 3395-3408, 1992.

[20] A. Baraldi and F. Parmiggiani, "An investigation of the textural characteristics associated with gray level cooccurrence matrix statistical parameters," IEEE Trans. on GeoRS, vol. 33, no. 2, pp. 293-304, March 1995.

[21] P. C. Smits and A. Annoni, "Updating land cover maps by using texture information from very high resolution space borne imagery," IEEE Trans. on GeoRS, vol. 37, no. 3, pp. 1244-1254, May 1999.

[22] V. Karathanassi, C. Iossifidis, and D. Rokos, "A texture-based classification method for classifying built areas according to their density," International Journal of Remote Sensing, vol. 21, no. 9, pp. 1807-1823, 2000. 
[23] M. A. Shaban and O. Dikshit, "Improvement of classification in urban areas by the use of textural features: The case study of Lucknow city, Uttar Pradesh," International Journal of Remote Sensing, vol. 22, no. 4, pp. 565-593, 2001.

[24] J. Cihlar, J. Okouneva, G. Beaubien, and R. Latifovic, "A new histogram quantization algorithm for land cover mapping," International Journal of Remote Sensing, vol. 22, no. 11, pp. 2151-2169, 2001.

[25] T. Parrinello and R. A. Vaughan, "Multifractal analysis and feature extraction in satellite imagery," International Journal of Remote Sensing, vol. 23, no. 9, pp. 1799-1825, 2002.

[26] R. L. Kettig and D. A. Landgrebe, "Classification of multispectral data by extraction and classification of homogenous objects," IEEE Trans. on Geoscience Electronics, vol. 14, no. 1, pp. 19-26, 1976.

[27] A. Baraldi and F. Parmiggiani, "Urban area classification by multispectral SPOT images," IEEE Trans. on GeoRS, vol. 28, no. 4, pp. 674-680, July 1990.

[28] L. Moller-Jensen, "Knowledge based classification of an urban area using texture and context information in Landsat-TM imagery," Photogrammetric Engineering and Remote Sensing, vol. 56, pp. 899-904, 1990.

[29] J. A. Richards, D. A. Landgrebe, and P. H. Swain, "A means for utilizing ancillary information in multispectral classification," Remote Sensing of Environment, vol. 12, pp. 463-477, 1982.

[30] A. Rosenfeld, R. A. Hummel, and S. W. Zucker, "Scene labeling by relaxation operations," IEEE Trans. on SMC, vol. 6, pp. 420-433, 1973.

[31] I. L. Thomas, "Spatial postprocessing of spectrally classified landsat data," Photogrammetric Engineering and Remote Sensing, vol. 46, pp. 1201-1206, 1980.

[32] K. S. Fu and T. S. Yu, Statistical Pattern Classification using Contextual Information, Research Studies Press, USA, 1980.

[33] J. C. Tilton, S. B. Vardeman, and P. H. Swain, "Estimation of context for statistical classification of multispectral data," IEEE Trans. on Geoscience Electronics, vol. 20, pp. 445-452, 1982.

[34] S. W. Wharton, "A contextual classification method for recognizing land use patterns in high resolution remotely sensed data," Pattern Recognition, vol. 15, pp. 317-324, 1982. 
[35] C. M. Gurney and J. R. G. Townshend, "The use of contextual information in the classification of remotely sensed data," Photogrammetric Engineering and Remote Sensing, vol. 49, pp. 55-64, 1983.

[36] J. L. Cushnie, "The interactive effect of spatial resolution and degree of internal variability within land cover types on classification accuracies," International Journal of Remote Sensing, vol. 8, no. 1, pp. 15-29, 1987.

[37] N. Khazenie and Crawford, "Spatial temporal autocorrelated model for contextual classfication," IEEE Trans. on GeoRS, vol. 28, no. 4, pp. 529-539, July 1990.

[38] T. Kusaka, H. Egava, and Y. Kawata, "Classification of the SPOT image using spectral and spatial features of primitive regions that have nearly uniform color," IEEE Trans. on GeoRS, vol. 28, no. 4, pp. 749-752, July 1990.

[39] K. Kim and M. M. Crawford, "Adaptive parametric estimation and classification of remotely sensed imagery using a pyramid structure," IEEE Trans. on GeoRS, vol. 29, no. 4, pp. 481-493, July 1991.

[40] F. G. Alonso and S. Lopez Soria, "Using contextual information to improve land use classification of satellite images in central Spain," International Journal of Remote Sensing, vol. 12, pp. 2227-2235, 1991.

[41] P. Gong and P. J. Howarth, "Frequency based contextual classification and graylevel vector reduction for land use identification," Photogrammetric Engineering and Remote Sensing, vol. 58, pp. 423-437, 1992.

[42] P. Gong and P. J. Howarth, "Land use classification of SPOT HRV data using a cover frequency method," International Journal of Remote Sensing, vol. 13, pp. 1459-1471, 1992.

[43] M. J. Barnsley and S. L. Barr, "Inferring urban land use from satellite sensor images using kernel-based spatial reclassification," Photogrammetric Engineering and Remote Sensing, vol. 62, pp. 949-958, 1996.

[44] A. Lobo, "Image segmentation and discriminant analysis for the identification of land cover units in ecology," IEEE Trans. on GeoRS, vol. 35, no. 5, pp. 1136-1145, September 1997.

[45] B. Kartikeyan, A. Sarkar, and K. L. Majumder, "A segmentation approach to classification of remote sensing imagery," International Journal of Remote Sensing, vol. 19, pp. 1695-1709, 1998. 
[46] K. M. S. Sharma and A. Sarkar, "A modified contextual classification technique for remote sensing data," Photogrammetric Engineering and Remote Sensing, vol. 64, pp. 273-280, 1998.

[47] S. Geman and D. Geman, "Stochastic relaxation, Gibbs distributions, and the Bayesian restoration of images," IEEE Trans. on PAMI, vol. 6, no. 6, pp. 721-741, November 1984.

[48] M. C. Zhang, R. M. Haralick, and J. B. Campbell, "Multispectral image context classification using stochastic relaxation," IEEE Trans. on SMC, vol. 20, no. 1, pp. 128-140, January 1990.

[49] B. Jeon and D. A. Landgrebe, "Classification with spatio temporal interpixel class dependency contexts," IEEE Trans. on GeoRS, vol. 30, no. 4, pp. 663-672, July 1992.

[50] Y. Jhung and P. H. Swain, "Bayesian contextual classification based on modified m-estimates and Markov Random Fields," IEEE Trans. on GeoRS, vol. 34, no. 1, pp. 67-75, January 1996.

[51] A. H. S. Solberg, T. Taxt, and A. K. Jain, "A Markov Random Field model for classification of multisource satellite imagery," IEEE Trans. on GeoRS, vol. 34, no. 1, pp. 100-113, January 1996.

[52] T. Yamazaki and D. Gingras, "Unsupervised multispectral image classification using MRF models and LVQ method," IEEE Trans. on GeoRS, vol. 37, no. 2, pp. 1173-1176, March 1999.

[53] B. C. K. Tso and P. M. Mather, "Classification of multisource remote sensing imagery using a genetic algorithm and Markov Random Fields," IEEE Trans. on GeoRS, vol. 37, no. 3, pp. 1255-1260, May 1999.

[54] I. B. Kerfoot and Y. Bresler, "Theoretical analysis of multispectral image segmentation criteria," IEEE Trans. on Image Processing, vol. 8, no. 6, pp. 798-820, June 1999.

[55] S. Yu, M. Berthod, and G. Giraudon, "Toward robust analysis of satellite images using map information: Application to urban area detection," IEEE Trans. on GeoRS, vol. 37, no. 4, pp. 1925-1939, July 1999.

[56] A. Sarkar, K. Biswas, B. Kartikeyan, V. Kumar, K. L. Majumder, and D. K. Pal, "A MRF model based segmentation approach to classification for multispectral imagery," IEEE Trans. on GeoRS, vol. 40, no. 5, pp. 1102-1113, May 2002. 
[57] M. I. Pedley and P. J. Curran, "Per-field classification: An example using SPOT HRV imagery," International Journal of Remote Sensing, vol. 12, pp. 2181-2192, 1991.

[58] F. Wang, "A knowledge-based vision system for detecting land changes at urban fringes," IEEE Trans. on GeoRS, vol. 31, no. 1, pp. 136-145, January 1993.

[59] S. De Bruin and B. G. H. Gorte, "Probabilistic image classification using geological map units applied to land cover change detection," International Journal of Remote Sensing, vol. 21, no. 12, pp. 2389-2402, 2000.

[60] S. De Bruin, "Predicting the areal extent of land cover types using classified imagery and geostatistics," Remote Sensing of Environment, vol. 74, pp. 387$396,2000$.

[61] S. M. J. Baban and K. W. Yusof, "Mapping land use/cover distribution on a mountainous tropical island using remote sensing and GIS," International Journal of Remote Sensing, vol. 22, no. 10, pp. 1909-1918, 2001.

[62] P. Jackson, Introduction to Expert Systems, Addison-Wesley Publ., Massachusets, third edition, 1999.

[63] S. W. Wharton, "A spectral knowledge-based approach for urban land-cover discrimination," IEEE Trans. on GeoRS, vol. 25, no. 3, pp. 272-282, May 1987.

[64] J. Ton, J. Sticklen, and A. K. Jain, "Knowledge based segmentation of Landsat images," IEEE Trans. on GeoRS, vol. 29, no. 2, pp. 222-232, March 1991.

[65] B. Kartikeyan, K. L. Majumder, and A. R. Dasgupta, "An expert system for land cover classification," IEEE Trans. on GeoRS, vol. 33, no. 1, pp. 58-66, January 1995.

[66] L. M. Jensen, "Classification of urban land cover based on expert systems, object models and texture," Comput., Environ. and Urban Systems, vol. 21, no. 3/4, pp. 291-302, 1997.

[67] J. A. M. Casasnovas, "A cartographic and database approach for land cover/use mapping and generalization from remotely sensed data," International Journal of Remote Sensing, vol. 21, no. 9, pp. 1825-1842, 2000.

[68] S. Barr and M. Barnsley, "Reducing structural clutter in land cover classifications of high spatial resolution remotely-sensed images for urban land use mapping," Computers and Geosciences, vol. 26, pp. 433-449, 2000. 
[69] W. L. Stefanov, M. S. Ramsey, and P. R. Christensen, "Monitoring urban land cover change: An expert system approach to land cover classification of semiarid to arid urban centers," Remote Sensing of Environment, vol. 77, pp. 173-185, 2001.

[70] M. Datcu, F. Melgani, A. Piardi, and S. B. Serpico, "Multisource data classification with dependence trees," IEEE Trans. on GeoRS, vol. 40, no. 3, pp. 609-617, March 2002.

[71] P. W. Mausel, W. J. Kramber, and J. K. Lee, "Optimum band selection for supervised classification of multispectral data," Photogrammetric Engineering and Remote Sensing, vol. 56, pp. 55-60, 1990.

[72] F. Tsai and W. D. Philpot, "A derivative aided hyperspectral image analysis system for land cover classification," IEEE Trans. on GeoRS, vol. 40, no. 2, pp. 416-425, February 2002.

[73] I. T. Joliffe, Principal Component Analysis, Springer-Verlag, New York, second edition, 2002.

[74] J. C. Harsanyi and C. I. Chang, "Hyperspectral image classification and dimensionality reduction: An orthogonal subspace projection approach," IEEE Trans. on GeoRS, vol. 32, no. 4, pp. 779-785, July 1994.

[75] X. Jia and J. A. Richards, "Segmented principal components transformation for efficient hyperspectral remote-sensing image display and classification," IEEE Trans. on GeoRS, vol. 37, no. 1, pp. 538-542, January 1999.

[76] T. M. Kuplich, C. C. Freitas, and J. V. Soares, "The study of ERS-1 SAR and Landsat TM synergism for land use classification," International Journal of Remote Sensing, vol. 21, no. 10, pp. 2101-2111, 2000.

[77] H. Ren and C. I. Chang, "A generalized orthogonal subspace projection approach to unsupervised multispectral image classification," IEEE Trans. on GeoRS, vol. 38, no. 6, pp. 2515-2528, November 2000.

[78] S. Kumar, J. Ghosh, and M. M. Crawford, "Best bases feature extraction algorithms for classification of hyperspectral data," IEEE Trans. on GeoRS, vol. 39, no. 7, pp. 1368-1379, July 2001.

[79] C. I. Chang, X. L. Zhao, M. L. G. Althouse, and J. J. Pan, "Least squares subspace projection approach to mixed pixel classification for hyperspectral images," IEEE Trans. on GeoRS, vol. 36, no. 3, pp. 898-912, May 1998. 
[80] C. I. Chang and C. M. Brumbley, "A Kalman filtering approach to multispectral image classification and detection of changes in signature abundance," IEEE Trans. on GeoRS, vol. 37, no. 1, pp. 257-268, January 1999.

[81] C. I. Chang and H. Ren, "An experiment-based quantitative and comparative analysis of target detection and image classification algorithms for hyperspectral imagery," IEEE Trans. on GeoRS, vol. 38, no. 2, pp. 1044-1063, March 2000.

[82] C. Small, "Estimation of urban vegetation abundance by spectral mixture analysis," International Journal of Remote Sensing, vol. 22, no. 7, pp. 13051334, 2001.

[83] S. Roessner, U. Heiden, and H. Kaufmann, "Automated differentiation of urban surface based on airborne hyperspectral imagery," IEEE Trans. on GeoRS, vol. 39, no. 7, pp. 1525-1532, July 2001.

[84] C. I. Chang, "Target signature-constrained mixed pixel classification for hyperspectral imagery," IEEE Trans. on GeoRS, vol. 40, no. 5, pp. 1065-1081, May 2002 .

[85] J. Verhoeye and R. D. Wulf, "Land cover mapping at sub-pixel using linear optimization techniques," Remote Sensing of Environment, vol. 79, pp. 96-104, 2002 .

[86] J.V. Kittler, M. Hatef, R.P.W. Duin, and J. Matas, "On combining classifiers," IEEE Trans. on PAMI, vol. 20, no. 3, pp. 226-239, March 1998.

[87] B. Jeon and D. A. Landgrebe, "Decision fusion approach for multitemporal classification," IEEE Trans. on GeoRS, vol. 37, no. 3, pp. 1227-1233, May 1999 .

[88] J. A. Benediktsson, J. R. Sveinsson, and P. H. Swain, "Hybrid consensus theoretical classification," IEEE Trans. on GeoRS, vol. 35, no. 4, pp. 833-843, July 1997.

[89] J. A. Benediktsson and I. Kanellopoulos, "Classification of multisource and hyperspectral data based on decision fusion," IEEE Trans. on GeoRS, vol. 37, no. 3, pp. 1367-1377, May 1999.

[90] L. O. Jimenez, A. M. Morell, and A. Creus, "Classification of hyperdimensional data based on feature and decision fusion approaches using projection pursuit, majority voting, and neural networks," IEEE Trans. on GeoRS, vol. 37, no. 3, pp. 1360-1366, May 1999. 
[91] B. M. Steele, "Combining multiple classifiers: An application using spatial and remotely sensed information for land cover type mapping," Remote Sensing of Environment, vol. 74, pp. 545-556, 2000.

[92] P. C. Smits, "Multiple classifier systems for supervised remote sensing image classification based on dynamic classifier selection," IEEE Trans. on GeoRS, vol. 40, no. 4, pp. 801-813, April 2002.

[93] A. H. S. Solberg, A. K. Jain, and T. Taxt, "Multisource classification of remotely sensed data: Fusion of Landsat TM and SAR images," IEEE Trans. on GeoRS, vol. 32, no. 4, pp. 768-778, July 1994.

[94] J. J. Clark and A. Yuille, Data Fusion for Sensory Information Processing Systems, Kluwer Academic Publishers, Boston, 1990.

[95] S. Sarkar and K. L. Boyer, Computing Perceptual Organization in Computer Vision, World Scientific, 1994.

[96] J. B. Burns, A. R. Hanson, and E. M. Riseman, "Extracting straight lines," IEEE Trans. on PAMI, vol. 8, no. 4, pp. 425-455, July 1986.

[97] S. Sarkar and K. L. Boyer, "On optimal infinite impulse response edge detection filters," IEEE Trans. on PAMI, vol. 13, no. 11, pp. 1154-1171, November 1991.

[98] S. G. Tan, "Image feature extraction: Line detection and organization," Msc. thesis, The Ohio State University, Autumn 1990.

[99] A. V. Oppenheim, R. W. Schafer, and J. R. Buck, Discrete Time Signal Processing, Prentice Hall Inc., second edition, 1999.

[100] D. M. Wuechser and K. L. Boyer, "Robust contour decomposition using a constant curvature criterion," IEEE Trans. on PAMI, vol. 13, no. 1, pp. 41-51, January 1991.

[101] R. Srikantiah, Flynn P. J., and K. L. Boyer, "Multiscale surface organiztion and description for free form object recognition," Computer Vision and Image Understanding, 2002.

[102] R. O. Duda, P. E. Hart, and D. G. Stork, Pattern Classification, Wiley Interscience, New York, second edition, 2001.

[103] H. Yamamoto, "A method of deriving compatibility coefficients for relaxation operators," Computer Vision and Image Processing, vol. 10, pp. 256-271, 1979.

[104] H. V. Poor, An Introduction to Signal Detection and Estimation, Springer Verlag, New York, second edition, 1994. 
[105] W. A. Sethares and T. W. Staley, "Periodicity transforms," IEEE Trans. on PAMI, vol. 47, no. 11, pp. 2953-2964, November 1999.

[106] R. J. Kauth and G. S. Thomas, "The tasselled cap - a graphic description of the spectral temporal development of agricultural crops as seen by LANDSAT," in Proceedings of the Symposium Machine Processing of Remote Sensing Data, LARS, Purdue, 1976.

[107] A. R. Huete, "A soil-adjusted vegetation index (SAVI)," Remote Sensing of Environment, vol. 25, pp. 295-309, 1988.

[108] D. Tanré, B. N. Holben, and Y. J. Kaufman, "Atmospheric correction algorithm for NOAA-AVHRR products: Theory and application," IEEE Trans. on GeoRS, vol. 30, no. 2, pp. 231-248, March 1992.

[109] Y. J. Kaufman and D. Tanré, "Atmospherically resistant vegetation index (ARVI) for EOS-MODIS," IEEE Trans. on GeoRS, vol. 30, no. 2, pp. 261-270, March 1992.

[110] H. Q. Liu and A. R. Huete, "A feedback modification of the ndvi to minimize canopy background and atmospheric noise," IEEE Trans. on GeoRS, vol. 33, no. 2, pp. 457-465, March 1995.

[111] A. R. Huete, H. Q. Liu, K. Batcily, and W. Van Leeuwen, "A comparison of vegetation indices over a global set of TM images for EOS-MODIS," Remote Sensing of Environment, vol. 59, pp. 440-451, 1997.

[112] A. R. Huete, C. Justice, and W. Van Leeuwen, "MODIS vegetation index (MOD13), EOS MODIS algorithm," Theoretical basis document, NASA Goddard Space Flight Center, Greenbelt, MD, 1996.

[113] B. Pinty and M. M. Verstraete, "GEMI: A nonlinear index to monitor from satellites," Vegetatio, vol. 101, pp. 15-20, 1992.

[114] M. M. Verstraete and B. Pinty, "Designing optimal spectral indexes for remote sensing applications," IEEE Trans. on GeoRS, vol. 34, no. 5, pp. 1254-1265, September 1996.

[115] G. Rondeaux, M. Steven, and F. Baret, "Optimization of soil-adjusted vegetation indices," Remote Sensing of Environment, vol. 55, pp. 95-107, 1996.

[116] K. McGwire, T. Minor, and L. Fenstermaker, "Hyperspectral mixture modeling for quantifying sparse vegetation cover in arid environments," Remote Sensing of Environment, vol. 72, pp. 360-374, 2000. 
[117] A. J. Elmore, J. F. Mustard, S. J. Manning, and D. B. Lobell, "Quantifying vegetation change in semiarid environments: Precision and accuracy of spectral mixture analysis and the normalized difference vegetation index," Remote Sensing of Environment, vol. 73, pp. 87-102, 2000.

[118] C. D. Elvidge and Z. Chen, "Comparison of broad-band and narrow-band red and near-infrared vegetation indices," Remote Sensing of Environment, vol. 54, pp. 38-48, 1995.

[119] A. R. Huete and H. Q. Liu, "An error and sensitivity analysis of the atmospheric- and soil-correcting variants of the NDVI for the MODIS-EOS," IEEE Trans. on GeoRS, vol. 32, no. 4, pp. 897-905, July 1994.

[120] V. Jayaraman, S. K. Srivastava, D. K. Raju, and U. R. Rao, "Total solution approach using IRS-1C and IRS-P3 data," IEEE Trans. on GeoRS, vol. 38, no. 1, pp. 587-604, January 2000.

[121] R. L. Lawrence and W. J. Ripple, "Comparisons among vegetation indices and bandwise regression in a highly disturbed, heterogeneous landscape: Mount St. Helens, Washington," Remote Sensing of Environment, vol. 64, pp. 91-102, 1998.

[122] M. Roderick, R. Smith, and S. Cridland, "The precision of the NDVI derived from AVHRR observations," Remote Sensing of Environment, vol. 56, pp. 5765, 1996.

[123] N. H. Broge and E. Leblanc, "Comparing prediction power and stability of broadband and hyperspectral vegetation indices for estimation of green leaf area index and canopy chlorophyll density," Remote Sensing of Environment, vol. 76, pp. 156-172, 2000.

[124] S. N. Goward, C. J. Tucker, and D. G. Dye, "North American vegetation patterns observed with the NOAA-7 advanced very high resolution radiometer," Vegetatio, vol. 64, pp. 3-14, 1985.

[125] C. J. Tucker and P. J. Sellers, "Satellite remote sensing of primary productivity," International Journal of Remote Sensing, vol. 7, pp. 1395-1416, 1986.

[126] J. P. Malingreau, C. J. Tucker, and N. Laporte, "AVHRR for monitoring global tropical deforestation," International Journal of Remote Sensing, vol. 10, pp. 855-867, 1989.

[127] J. R. G. Townshend, C. O. Justice, and D. Skole, "The $1 \mathrm{~km}$ resolution global data set: Needs of the international geosphere programme," International Journal of Remote Sensing, vol. 15, pp. 3417-3441, 1994. 
[128] R. B. Myneni, G. Asrar, D. Tanré, and B. J. Choudhury, "Remote sensing of solar radiation absorbed and reflected by vegetated land surfaces," IEEE Trans. on GeoRS, vol. 30, no. 2, pp. 302-314, March 1992.

[129] P. S. Thenkabail, R. B. Smith, and E. De Pauw, "Hyperspectral vegetation indices and their relationships with agricultural crop characteristics," Remote Sensing of Environment, vol. 71, pp. 158-182, 2000.

[130] G. B. Senay and R. L. Elliot, "Combining AVHRR-NDVI and landuse data to describe temporal and spatial dynamics of vegetation," Forest Ecology and Management, vol. 128, pp. 83-91, 2000.

[131] T. N. Carlson and D. A. Ripley, "On the relation between NDVI, fractional vegetation cover, and leaf area index," Remote Sensing of Environment, vol. 62, pp. 241-252, 1997.

[132] C. O. Justice, J. R. G. Townshend, B. N. Holben, and C. J Tucker, "Analysis of the phenology of global vegetation using meteorological satellite data," International Journal of Remote Sensing, vol. 6, pp. 1271-1318, 1985.

[133] J. R. G. Townshend, C. Justice, W. Li, C. Gurney, and J. McManus, "Global land cover classification by remote sensing: Present capabilities and future possibilities," Remote Sensing of Environment, vol. 35, pp. 243-256, 1991.

[134] V. Caselles and E. Valor, "Mapping land surface emmisivity from NDVI: Application to European, African and South American areas," Remote Sensing of Environment, vol. 57, pp. 167-184, 1996.

[135] J. Symnazik and R. R. Griffiths, L. Gillies, "Visual exploration of satellite images," in Proceedings of the Statistical Computing Section and Section on Statistical Graphics, 2000, pp. 10-19.

[136] Y. J. Kaufman and L. A. Remer, "Detection of forests using mid-ir reflectance: An application for aerosol studies," IEEE Trans. on GeoRS, vol. 32, no. 3, pp. 672-683, May 1994.

[137] T. Fung, "Environmental quality and changes: A view from NDVI in Hong Kong," in Proceedings of IGARSS, August 1997, vol. 2, pp. 836-838.

[138] J. M. C. Pereira, "A comparative evaluation of NOAA/AVHRR vegetation indexes for burned surface detection and mapping," IEEE Trans. on GeoRS, vol. 37, no. 1, pp. 217-226, January 1999.

[139] P. R. Coppin and M. E. Bauer, "Processing of multitemporal Landsat TM imagery to optimize extraction of forest cover change features," IEEE Trans. on GeoRS, vol. 32, no. 4, pp. 918-927, July 1994. 
[140] D. F. Lozano-Garcia, R. N. Fernández, and C. J. Johannsen, "Assesment of regional biomass-soil relationships using vegetation indexes," IEEE Trans. on GeoRS, vol. 29, no. 2, pp. 331-339, March 1991.

[141] T. Ishiyama, S. Tanaka, K. Uchida, S. Fujikawa, Y. Yamsahita, and M. Kato, "Relationship among vegetation variables and vegetation features of arid lands derived from satellite data," Adv. Space Research, vol. 28, no. 1, pp. 183-188, 2001.

[142] P. M. Teillet, K. Staenz, and D. J. Willimas, "Effects of spectral, spatial and radiometric characteristics on remote sensing vegetation indices of forested regions," Remote Sensing of Environment, vol. 61, pp. 139-149, 1997.

[143] F. N. Kogan, "Application of vegetation index and brightness temperature for drought detection," Adv. Space Research, vol. 15, no. 11, pp. 91-100, 1995.

[144] P. J. Sellers, C. J. Tucker, and G. J. Collatz, "A global $10 \times 10$ NDVI data set for climate studies. part2-the adjustment of the NDVI and generation of global fields of terrestrial biophysical parameters," International Journal of Remote Sensing, vol. 15, pp. 3519-3545, 1994.

[145] Z. Li and M. Kafatos, "Interannual variability of vegetation in the United States and its relation to El Nino/Sothern Oscillation," Remote Sensing of Environment, vol. 71, pp. 239-247, 2000.

[146] J. C. Price, "Estimating leaf area index from satellite data," IEEE Trans. on GeoRS, vol. 31, no. 3, pp. 727-734, May 1993.

[147] W. C. Bausch and J. C. Price, "Leaf area index estimation from visible and near infrared reflectance data," Remote Sensing of Environment, vol. 52, pp. $55-65,1995$.

[148] A. Bégué, "Leaf area index, intercepted photosynthetically active radiation, and spectral vegetation indices: A sensitivity analysis for regular-clumped canopies," Remote Sensing of Environment, vol. 46, pp. 45-59, 1993.

[149] D. G. Brown, "A spectral unmixing approach to leaf area index (LAI) estimation at the Alpine treeline ecotone," in GIS and remote sensing applications in biogeography and ecology, A. Millington, S. J. Walsh, and P. Osborne, Eds., pp. 7-22. Dordrecht: Kluwer, 2001.

[150] C. L. Wiegand, S. J. Mass, J. K. Aase, J. L. Hatfield, P. J. Jr. Pinter, R. D. Jackson, E. T. Kanemasu, and R. L. Lapitan, "Multisite analysis of spectralbiophysical data for wheat," Remote Sensing of Environment, vol. 42, pp. 1-21, 1992. 
[151] T. Miura, A. R. Huete, and H Yoshioka, "Evaluation of sensor calibration uncertainties on vegetation indices for MODIS," IEEE Trans. on GeoRS, vol. 38, no. 3, pp. 1399-1409, May 2000.

[152] R. K. Gupta, T. S. Prasad, and D. Vijayan, "Relationship between LAI and NDVI for IRS LISS and LANDSAT TM bands," Adv. Space Research, vol. 26, no. 7 , pp. 1047-1050, 2000.

[153] C. A. Shull, "A spectrophotometric study of reflection of light from leaf surfaces," Botanical Gazette, vol. 87, no. 5, pp. 583-607, June 1929.

[154] G. S. Rabideau, C. S. French, and A. S. Holt, "The absorption and reflection spectra of leaves, chloroplast suspensions, and chloroplast fragments as measured in and Ulbricht sphere," American Journal of Botany, vol. 33, no. 10, pp. 769-777, December 1946.

[155] W. D. Billings and R. J. Morris, "Reflection of visible and infrared radiation from leaves of different ecological groups," American Journal of Botany, vol. 38, no. 5, pp. 327-331, May 1951.

[156] C. A. Federer and C. B. Tanner, "Spectral distribution of light in the forest," Ecology, vol. 47, no. 4, pp. 555-560, June 1966.

[157] D. M. Gates, J. C. S. Keegan, and V. R. Weidner, "Spectral properties of plants," Applied Optics, vol. 4, no. 1, pp. 11-20, January 1965.

[158] C. F. Jordan, "Derivation of leaf area index quality of light on the forest floor," Ecology, vol. 50, no. 4, pp. 663-666, July 1969.

[159] J. E. Colwell, "Vegetation canopy reflectance," Remote Sensing of Environment, vol. 3, pp. 175-183, 1974.

[160] J. E. Colwell, Bidirectional spectral reflectance of grass canopies for determination of above ground standing biomass, Ph.D. thesis, University of Michigan, 1973.

[161] R. F. Nalepka, C. E. Colwell, and D. P. Rice, "Forecasts of winter wheat yield and production using LANDSAT data," Final Report 114800-38-F, NASA CR/ERIM, 1977.

[162] J. W. Rouse, R. H. Haas, J. A. Schell, D. W. Deering, and J. C. Harlan, "Monitoring the vernal advancement of natural vegetation," Final report, NASA/GCSFC, Greenbelt, MD, 1974.

[163] C. J. Tucker, "Photocombinations for monitoring vegetation," Remote Sensing of Environment, vol. 8, pp. 127-150, 1979. 
[164] D. W. Deering, Rangeland reflectance characteristics measured by aircraft and spacecraft sensors, Ph.D. thesis, Texas AM University, College Station, Texas, 1978.

[165] R. D. Jackson, "Spectral indices in n-space," Remote Sensing of Environment, vol. 13, pp. 409-421, 1983.

[166] G. Strang, Linear Algebra and Its Applications, Harcourt College Publishers, Florida, USA, third edition, 1986.

[167] V. Klemas, "Remote sensing of estuaries: An overview," IEEE Ocean, pp. 302-309, 1986.

[168] A. Niedermeier, E. Romaneessen, and S. Lehner, "Detection of coastlines in SAR images using wavelet methods," IEEE Trans. on GeoRS, vol. 38, no. 5, pp. 2270-2281, September 2000.

[169] T. J. McIntire and J. J. Simpson, "Arctic sean ice, cloud, water and lead classification using neural networks and 1.6- $\mu \mathrm{m}$ data," IEEE Trans. on GeoRS, vol. 40, no. 9, pp. 1956-1972, September 2002.

[170] C Wang, L. Huang, and A. Rosenfeld, "Detecting clouds and cloud shadows on aerial photographs," Pattern Recognition Letters, pp. 55-64, 1991.

[171] A. T. S. Ho, "Detecting coluds and cloud shadows in multispectral satellite images for tropical areas," in Proceedings of IPA, July 1997, pp. 848-851.

[172] M. F. Jasinski, "Estimation of subpixel vegetation density of natural regions using satellite multispectral imagery," IEEE Trans. on GeoRS, vol. 34, no. 5, pp. 804-813, May 1996.

[173] J. J. Simpson and J. R. Stitt, "A procedure for the detection and removal of cloud shadow from AVHRR data over land," IEEE Trans. on GeoRS, vol. 36, no. 3, pp. 880-897, May 1998.

[174] G. Lisens, P. Kempencers, F. Fierens, and J. Van Rensbergen, "Development of cloud, snow, and shadow masking algorithms for vegetation imagery," in Proceedings of IGARSS, 2000, vol. 2, pp. 834-836.

[175] J. J. Simpson, Z. Jin, and J. R. Stitt, "Cloud shadow detection under arbitrary viewing and illumination conditions," IEEE Trans. on GeoRS, vol. 38, no. 2, pp. 972-976, March 2000.

[176] H. Spitzer, R. Franck, M. Kollewe, N. Rega, A. Rothkirch, and R. Wiemker, "Change detection with $1 \mathrm{~m}$ resolution satellite and aerial images," in Proceedings of IGARSS, 2001, vol. 5, pp. 2256-2258. 
[177] T. E. Avery and G. L. Berlin, Fundamentals of Remote Sensing and Airphoto Interpretation, Macmillan Publishing, fifth edition, 1992.

[178] S. Sarkar and K. L. Boyer, "Quantitative measures of change based on feature organization: Eigenvalues and Eigenvectors," Computer Vision and Image Understanding, vol. 71, pp. 110-136, 1998.

[179] C. Berge, The Theory of Graphs, Dover Publications, New York, 2001.

[180] B. Bollobas, Random Graphs, Cambridge University Press, Cambridge, second edition, 2001.

[181] L. S. Shapiro and J. M. Brady, "Feature-based correspondence: An eigenvector approach," Image and Vision Computing, vol. 10, pp. 283-288, 1992.

[182] J. Shi and J. Malik, "Normalized cuts and image segmentation," IEEE Trans. on PAMI, vol. 22, no. 8, pp. 888-905, August 2000.

[183] B. Mohar, "The Laplacian spectrum of graphs," in Proceedings of the Sixth Quadrennial Conference on the Theory and Applications of Graphs, 1998, vol. 2, pp. 871-898.

[184] M. Fiedler, "Algebraic connectivity of graphs," Czecholoslovak Mathematical Journal, vol. 98, pp. 298-305, 1973.

[185] M. Fiedler, "A property of eigenvectors of nonnegative symmetric matrices and its application to graph theory," Czecholoslovak Mathematical Journal, vol. 100, pp. 619-633, 1975.

[186] M. Fiedler, "Laplacian of graphs and algebraic connectivity," in Combinatorics and Graph Theory, 1989, vol. 25, pp. 57-70.

[187] P. Perona and W. Freeman, "A factorization approach to grouping," in ECCV98, 1998, pp. 655-670.

[188] Y. Weiss, "Segmentation using eigenvectors: A unifying view," in ICCV99, 1999, pp. 975-982.

[189] Y. Gdalyahu, D. Weinshall, and M. Werman, "Self-organization in vision: Stochastic clustering for image segmentation; perceptual grouping and image database organization," IEEE Trans. on PAMI, vol. 23, no. 10, pp. 1053-1074, October 2001.

[190] M. Pavan and M. Pelillo, "A new graph-theoretic approach to clustering and segmentation," in CVPR03, 2003, pp. 145-152. 
[191] E. P. Wigner, "Characteristic vectors of bordered matrices with infinite dimensions," Annals of Mathematics, vol. 62, pp. 548-564, 1955.

[192] E. P. Wigner, "Characteristic vectors of bordered matrices with infinite dimensions ii," Annals of Mathematics, vol. 65, pp. 203-207, 1957.

[193] E. P. Wigner, "On the distribution of the roots of certain symmetric matrices," Annals of Mathematics, vol. 67, pp. 325-327, 1958.

[194] L. Arnold, "On the asymptotic distribution of the eigenvalues of random matrices," Journal of Mathematical Analysis and Applications, vol. 20, pp. 262-268, 1967.

[195] L. Arnold, "Deterministic version of Wigner's semicircle law for the distribution of matrix eigenvalues," Linear Algebra and Its Applications, vol. 13, pp. 185$199,1976$.

[196] J. Shen, "On the singular values of Gaussian random matrices," Linear Algebra and Its Applications, vol. 326, pp. 1-14, 2001.

[197] I. J. Farkas, I. Derenyi, A. L. Barabasi, and T. Vicsek, "Spectra of "real-world" graphs: beyond the semicircle law," Physical Review E, vol. 64, pp. 1-12, 2001.

[198] D. J. Watts and S. H. Strogatz, "Collective dynamics of 'small-world' networks," Nature, vol. 393, pp. 440-442, 1998.

[199] D. J. Watts, Small Worlds, The Dynamics of Networks between Order and Randomness, Princeton University Press, New Jersey, 1999.

[200] I. Gutman and O. E. Polansky, Mathematical Concepts in Organic Chemistry, Springer Verlag, Berlin, 1986.

[201] D. Cvetkovic, M. Doob, I. Gutman, and A. Torgasev, Recent Results in the Theory of Graph Spectra, Elsevier Science Publishers, New York, 1988.

[202] H. Mayer, "Automatic object extraction from aerial imagery - A survey focusing on buildings," Computer Vision and Image Understanding, vol. 74, pp. 138-149, 1999.

[203] J. A. Shufelt, "Performance evaluation and analysis of monocular building extraction from aerial imagery," IEEE Trans. on PAMI, vol. 21, no. 4, pp. 311-326, April 1999.

[204] A. Huertas and R. Nevatia, "Detecting buildings in aerial images," Computer Vision, Graphics and Image Processing, vol. 41, pp. 131-152, 1988. 
[205] J. Shufelt and D. M. McKeown, "Fusion of monocular cues to detect man made structures in aerial imagery," CVGIP: Image Understanding, vol. 57, pp. 307-330, 1993.

[206] S. Krishnamachari and R. Chellappa, "Delineating buildings by grouping lines with MRFs," IEEE Trans. on IP, vol. 5, no. 1, pp. 164-168, January 1996.

[207] M. A. Maloof, P. Langley, S. Sage, and T. Binford, "Learning to detect rooftops in aerial images," in Proceedings of the 1997 Image Understanding Workshop DARPA97, 1997, pp. 835-845.

[208] A. Brunn and U. Weidner, "Hierarchical Bayesian nets for building extraction using dense digital surface models," Journal of Photogrammetry and Remote Sensing, vol. 53, pp. 296-307, 1998.

[209] Y. Zhang, "Optimisation of building detection in satellite images by combining multispectral classification and texture filtering," Journal of Photogrammetry and Remote Sensing, vol. 54, pp. 50-60, 1999.

[210] T. Kim and J.P. Muller, "Development of a graph based approach for building detection," Image and Vision Computing, vol. 17, no. 1, pp. 3-14, January 1999.

[211] A. Stassopoulou and T. Caelli, "Building detection using Bayesian networks," International Journal of Pattern Recognition and Artificial Intelligence, vol. 14, no. 6, pp. 715-733, 2000.

[212] P. Gamba, B. Houshmand, and M. Saccani, "Detection and extraction of buildings from iterferometric SAR data," IEEE Trans. on GeoRS, vol. 38, no. 1, pp. 611-618, January 2000.

[213] S. Noronha and R. Nevatia, "Detection and modeling of buildings from multiple aerial images," IEEE Trans. on PAMI, vol. 23, no. 5, pp. 501-518, May 2001.

[214] M. Fradkin, H. Maitre, and M. Roux, "Building detection from multiple aerial images in dense urban areas," Computer Vision and Image Understanding, vol. 82, pp. 181-207, 2001.

[215] P. Krishnamoorthy, K. L. Boyer, and P. J. Flynn, "Robust detection of buildings in digital surface models," in Proceedings of the International Conference on Pattern Recognition, August 2002, vol. 1, pp. 159-163.

[216] C. Lin and R. Nevatia, "Building detection and description from a single intensity image," Computer Vision and Image Understanding, vol. 72, no. 2, pp. 101-121, November 1998. 
[217] R. T. Collins, C. O. Jaynes, Y. Q. Cheng, X. Wang, F. Stolle, E. M. Riseman, and A. R. Hanson, "The Ascender system: Automated site modeling from multiple aerial images," Computer Vision and Image Understanding, vol. 72, pp. 143-162, 1998.

[218] H. Mayer, A. Baumgartner, and C. Steger, "Road extraction from aerial imagery," in CV-Online, 1998.

[219] R. Bajcsy and M. Takavoli, "Computer recognition of roads from satellite pictures," IEEE Trans. on SMC, vol. 6, no. 9, pp. 623-637, September 1976.

[220] F. Wang and R. Newkirk, "A knowledge based system for highway network extraction," IEEE Trans. on GeoRS, vol. 26, no. 5, pp. 525-531, September 1988.

[221] R. Fiset and F. Cavayas, "Automatic comparison of a topographic map with remotely sensed images in a map updating perspective: the road network case," International Journal of Remote Sensing, vol. 18, no. 4, pp. 991-1006, 1997.

[222] N. S. Netanyahu, V. Philomin, A. Rosenfeld, and A. J. Stromberg, "Robust detection of straight and circular road segments in noisy aerial images," Pattern Recognition, vol. 30, no. 10, pp. 1673-1686, 1997.

[223] F. Tupin, H. Maitre, J. F. Mangin, J. M. Nicolas, and E. Pechersky, "Detection of linear features in SAR images: Application to road network extraction," IEEE Trans. on GeoRS, vol. 36, no. 2, pp. 434-453, March 1998.

[224] V. Karathanassi, C. Iossifidis, and D. Rokos, "A thinning based method for recognizing and extracting peri-urban road networks from SPOT panchromatic images," International Journal of Remote Sensing, vol. 20, no. 1, pp. 153-168, 1999.

[225] I. Laptev, H. Mayer, T. Lindeberg, W. Eckstein, C. Steger, and A. Baumgartner, "Automatic extraction of roads from aerial images based on scale space and snakes," Machine Vision and Applications, vol. 12, pp. 23-31, 2000.

[226] B. K. Jeon, J. H. Jang, and K. S. Hong, "Road detection in spaceborne SAR images using a genetic algorithm," IEEE Trans. on GeoRS, vol. 40, no. 1, pp. 22-29, January 2002.

[227] W. Shi and C. Zhu, "The line segment match method for extracting road network from high-resolution satellite images," IEEE Trans. on GeoRS, vol. 40, no. 2, pp. 511-514, February 2002. 
[228] M. Nagao, T. Matsuyama, and Y. Ikeda, "Region extraction and shape analysis in aerial photographs," Computer Vision, Graphics and Image Processing, vol. 10, pp. 195-223, 1979.

[229] M. Nagao and T. Matsuyama, A Structural Analysis of Complex Aerial Photographs, Plenum Press, New York, USA, 1980.

[230] V. S. Hwang, L. S. Davis, and T. Matsuyama, "Hypothesis integration in image understanding systems," Computer Vision, Graphics and Image Processing, vol. 36, pp. 321-371, 1986.

[231] J. A. Hartigan, Clustering Algorithms, John Wiley and Sons, New York, 1975.

[232] A. K. Jain, R. P. W. Duin, and J. Mao, "Statistical pattern recognition: A review," IEEE Trans. on PAMI, vol. 22, no. 1, pp. 4-37, January 2000.

[233] N. Otsu, "A threshold selection method from gray-level histograms," IEEE Trans. on SMC, vol. 9, pp. 62-66, 1979.

[234] R. Adams and L. Bischof, "Seeded region growing," IEEE Trans. on PAMI, vol. 16, no. 6, pp. 641-647, June 1994.

[235] C. Godsil and G. Boyle, Algebraic Graph Theory, Springer-Verlag Inc., New York, 2001.

[236] M. Kass, A. Witkin, and D. Terzopoulos, "Snakes: Active contour models," International Journal of Computer Vision, pp. 321-331, 1988.

[237] R. C. Gonzales and R. E. Woods, Digital Image Processing, Addison Wesley, Mass., 1993.

[238] R. A. Brualdi, Introductory Combinatorics, Prentice Hall Inc., New Jersey, third edition, 1999.

[239] M. P. Do Carmo, Differential Geometry of Curves and Surfaces, Prentice-Hall Inc., New Jersey, 1976.

[240] J. R. Evans and E. Minieka, Optimization Algorithms for Networks and Graphs, Dekker Inc., New York, second edition, 1992.

[241] D. M. McKeown and J. L. Denlinger, "Cooperative methods for road tracking in aerial imagery," in Proceedings of CVPR1988, 1988, pp. 662-672. 